\title{
Inter-Organisational Knowledge Sharing in the Public Sector: The Role of Social Capital and Information and Communication Technology
}

By

\section{Sally Jansen van Vuuren}

A thesis submitted to the Victoria University of Wellington in fulfilment of the requirements for the degree of

Doctor of Philosophy in Information Systems

Victoria University of Wellington

2011 


\section{Abstract}

This thesis explores the dynamics of knowledge sharing, through the context of interorganisational collaboration in the public sector. The growth of collaboration across public sector organisations places increasing importance on knowledge sharing, yet it is an area that has historically proved difficult for the sector. Knowledge sharing research increasingly emphasises the importance of social capital in facilitating and influencing knowledge sharing behaviours, yet the public sector's approach to knowledge sharing has predominantly focussed on the use of information and communication technology (ICT) tools. The aim of this research is to better understand the dynamics of knowledge sharing, and the roles that social capital and ICT play in knowledge sharing.

This is a contemporary and important research topic. Public sector commitment to increasing collaboration requires a strong focus on inter-organisational knowledge sharing. This multiple-case, multi-method research incorporates a research design that blends qualitative and quantitative data collection and analysis. The research extends and deepens current understanding of the dynamics of knowledge sharing and contributes to the existing body of knowledge in the areas of knowledge sharing, social capital, and ICT. It also provides valuable empirical evidence relating to public sector based sharing at the inter-organisational level.

The research found that social capital is a significant concern for collaborative teams. Factors such as trust, group identity and shared purpose and goals were identified as important considerations for team members. Despite the sector's focus on ICT tools as key knowledge sharing mechanisms, the actual availability of tools was limited. Further, individuals' use of tools was low due to their perceptions of the available tools as difficult to use and of limited usefulness.

A key outcome of the research was the development of a conceptual framework that can be used to support the work of academics and practitioners engaged in the field of collaboration and knowledge sharing. The framework identifies six important antecedents that influence the development of social capital, and the availability and use of ICT. These antecedents include collaboration design, leadership, the perceived ease of use and perceived usefulness of ICT, and individual technical ability. The final antecedent identifies individual's commitment to the 'intrinsic value of public sector work' as a key collaboration factor. 
This thesis is dedicated to:

Diana Pryde whose personal and professional achievements are a constant source of inspiration. A role model and mentor to so many, but cherished by none more than me. Thank you for standing by my side.

And to

Cecil, my much loved father-in-law. A man of indomitable spirit and continuing courage in his battle with cancer. Kia Kaha dearest Pa.

\section{Acknowledgements}

My immense thanks go to Professor Sid Huff, for his guidance, support and patience in helping me to navigate the $\mathrm{PhD}$ process. His attention to detail and critical insight were invaluable in enabling me to complete this thesis. My thanks also to Dr. David Pauleen, whose knowledge and dedication to KM helped develop my interest in the field.

To my husband Pieter, whose hair turned grey during the $\mathrm{PhD}$ process - thank you for never giving up on me, even when times were really tough. I am so proud of all that you have achieved over the last few years, academically, professionally and personally. I support you in all that you do.

I am extremely grateful to the organisations that showed interest in my research, and granted access to the inter-organisational teams detailed in this research. My thanks also to the individuals in those teams - for your time, energy and candid comments.

Thanks also go to my peers; there is none who better understand the trials and tribulations as those who are standing in the trenches.

Finally, my thanks also to the Foundation of Research, Science and Technology (FRST) for funding this research through the Top Achiever Scholarship. 


\section{Table of Contents}

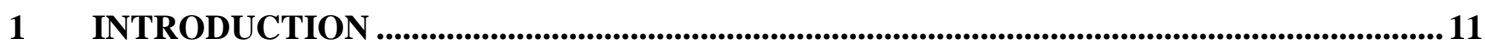

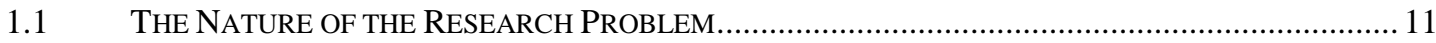

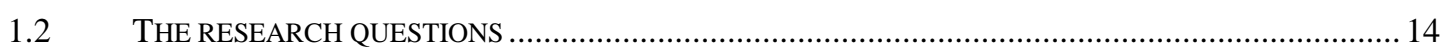

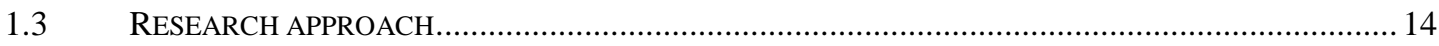

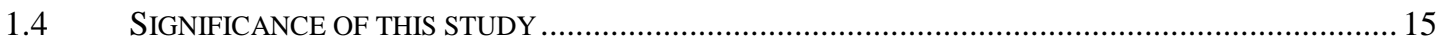

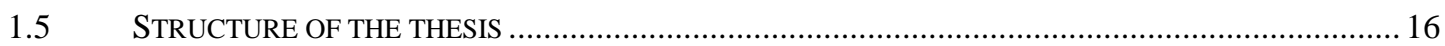

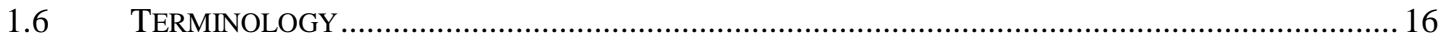

2 LITERATURE REVIEW AND CONCEPTUAL FRAMEWORK .......................................... 18

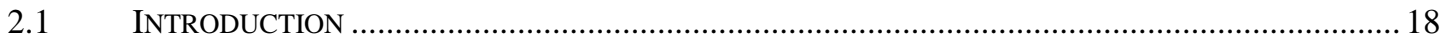

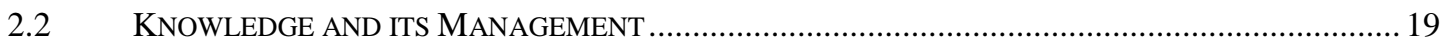

2.2.1 Epistemological foundations of knowledge ........................................................................ 19

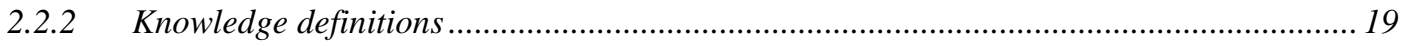

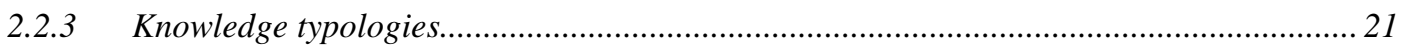

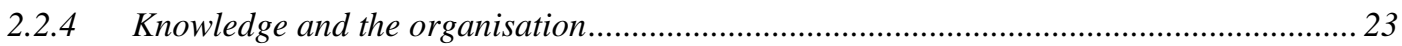

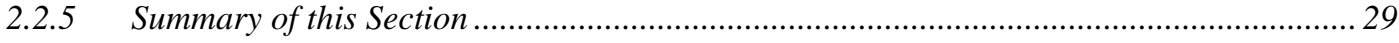

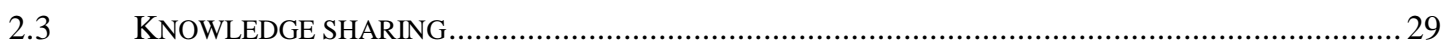

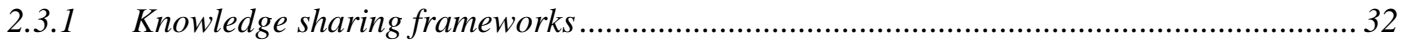

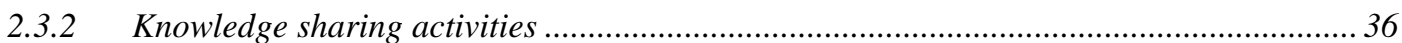

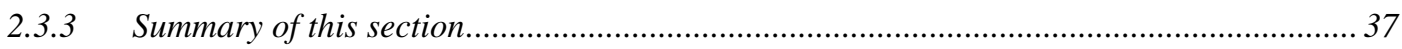

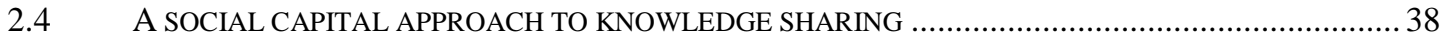

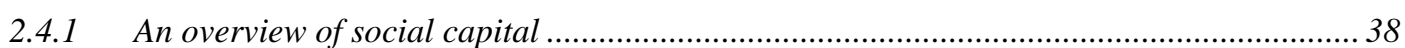

2.4.2 Conceptualisations of social capital and knowledge sharing ......................................... 39

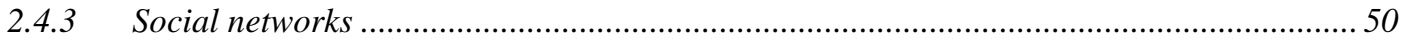

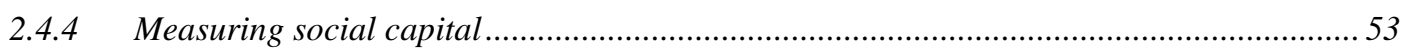

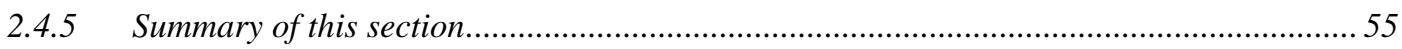

2.5 A TECHNOLOGICAL APPROACH TO KNOWLEDGE SHARING ….............................................55

2.5.1 ICT as a communication channel ............................................................................... 57

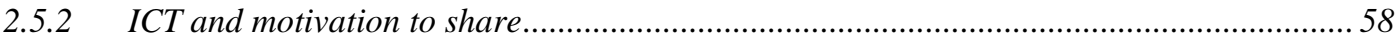

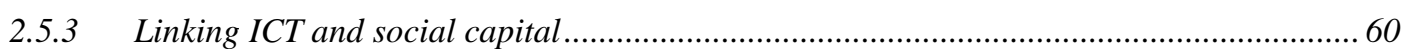

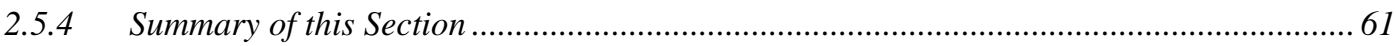

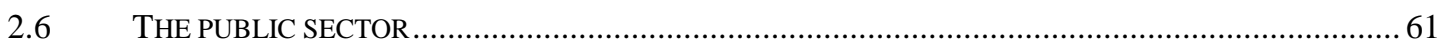

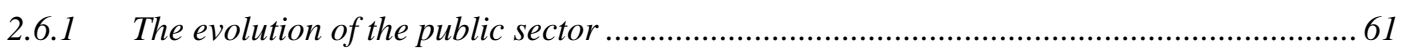

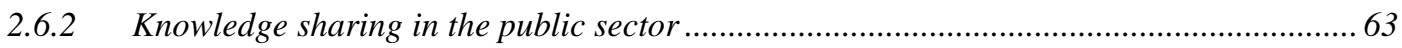

2.6.3 Barriers to knowledge sharing in the public sector........................................................... 65

2.6.4 Knowledge sharing in the New Zealand public sector ...................................................6 67

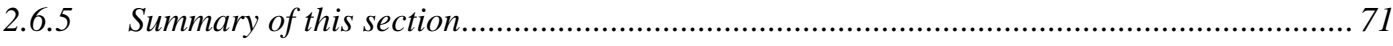

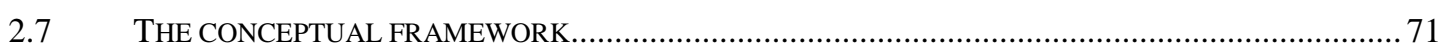

2.7.1 The purpose of a conceptual framework ......................................................................... 71 
2.7.2 Development of the conceptual framework in this study ............................................. 72

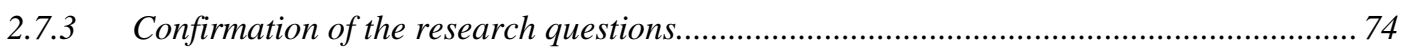

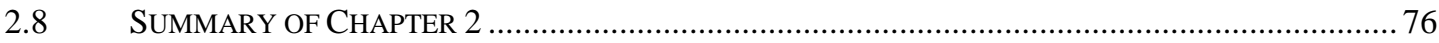

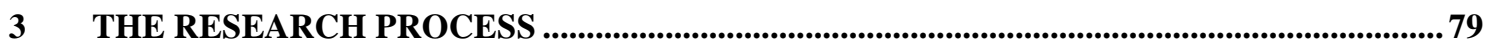

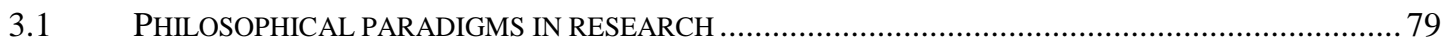

3.1.1 Positivist and post-positivist paradigms.................................................................. 82

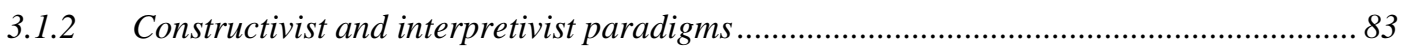

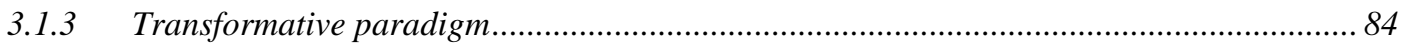

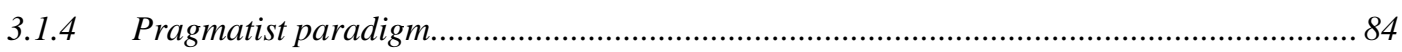

3.1.5 Justification of the philosophical stance adopted in this study ..................................... 85

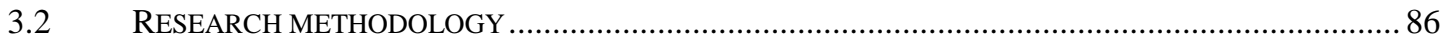

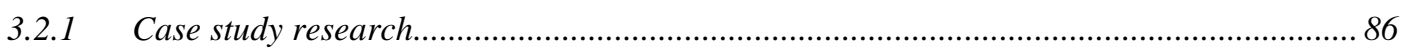

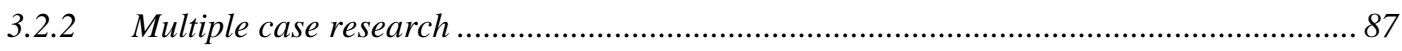

3.2.3 Justification of case research methodology.................................................................... 88

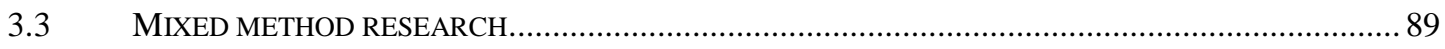

3.3.1 An overview of mixed methods design ....................................................................... 89

3.3.2 The mixed methods design of this study........................................................................... 91

3.3.3 Addressing qualitative data validity issues .............................................................. 93

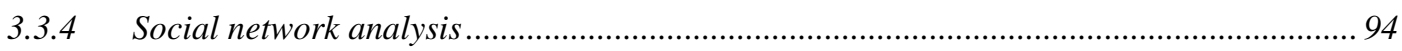

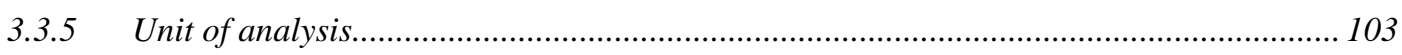

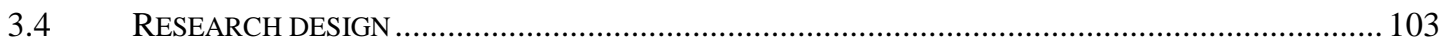

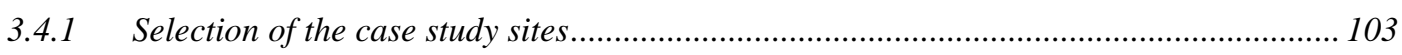

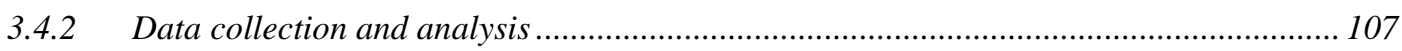

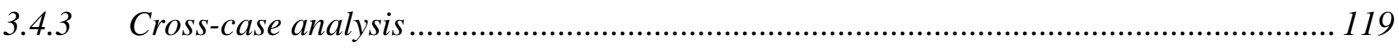

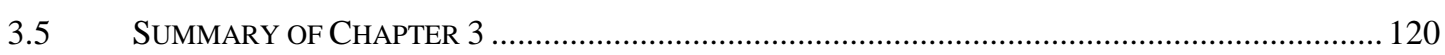

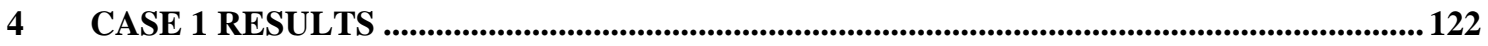

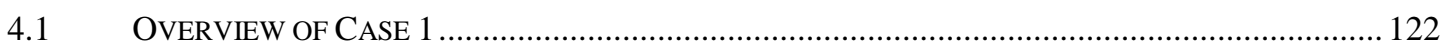

4.2 PERCEPTIONS OF KNOWLEDGE …............................................................................. 126

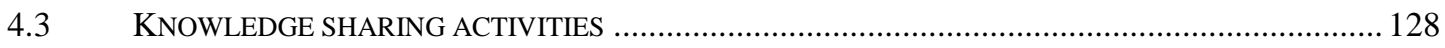

4.3.1 Choice of communication channel for knowledge sharing .............................................. 130

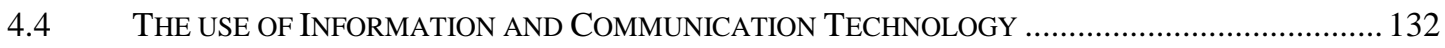

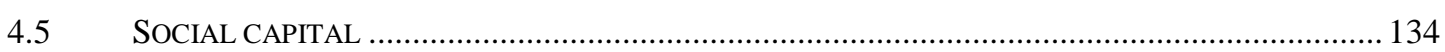

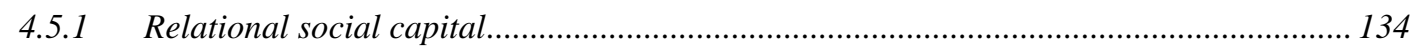

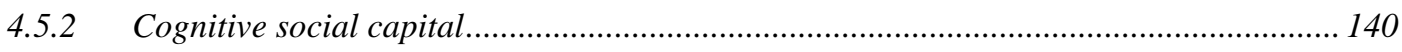

4.5.3 Structural social capital ......................................................................................... 143

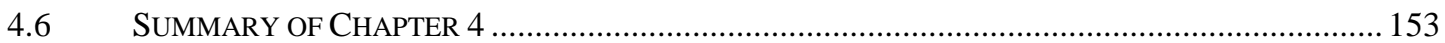

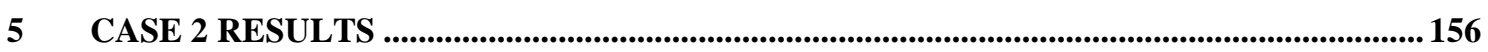

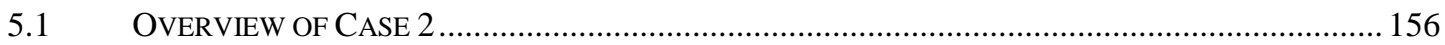


5.3 KNOWLEDGE SHARING ACTIVITIES 160

5.3.1 Choice of communication channel for knowledge sharing ........................................... 163

5.4 THE USE OF INFORMATION AND COMMUNICATION TECHNOLOGY ........................................ 165

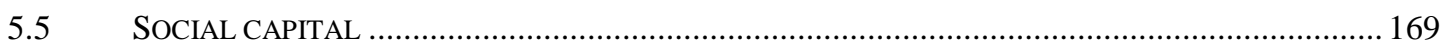

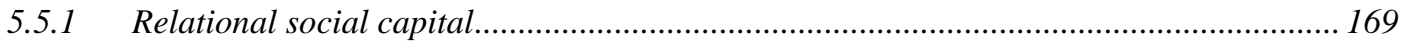

5.5.2 Cognitive social capital................................................................................................ 174

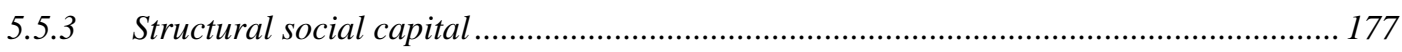

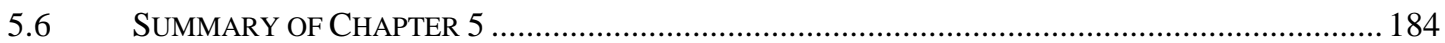

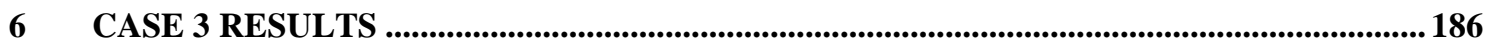

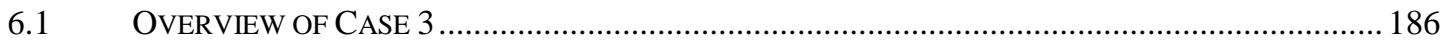

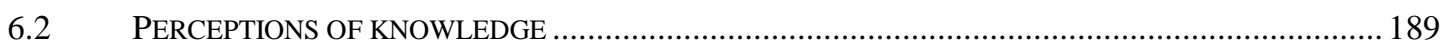

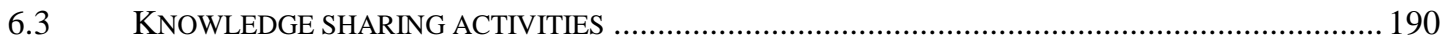

6.3.1 Choice of communication channel for knowledge sharing............................................. 192

6.4 THE USE OF INFORMATION AND COMMUNICATION TECHNOLOGY (ICT)............................ 193

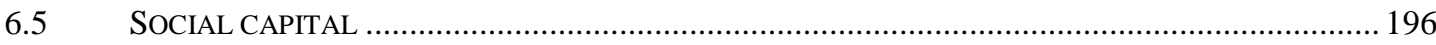

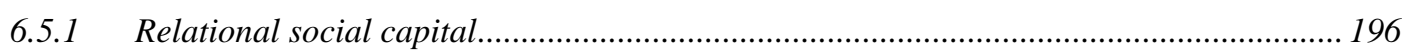

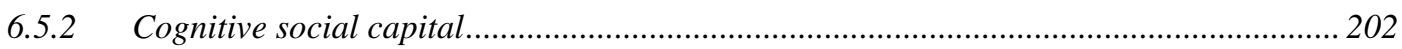

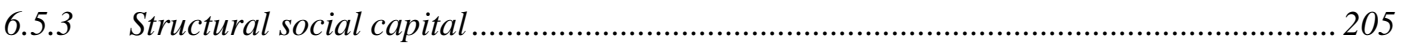

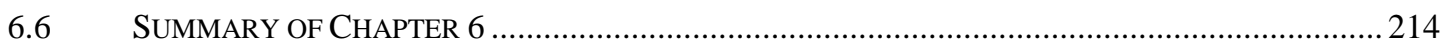

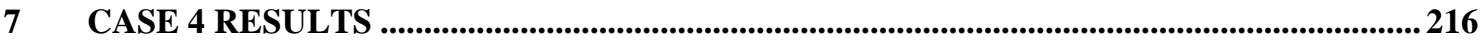

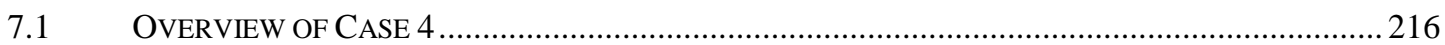

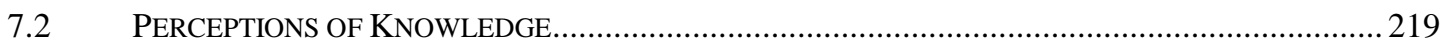

KNOWLEDGE SHARING ACTIVITIES ............................................................................ 220

7.3.1 Choice of communication channel for knowledge sharing........................................... 222

7.4 THE USE OF INFORMATION AND COMMUNICATION TECHNOLOGY ....................................224

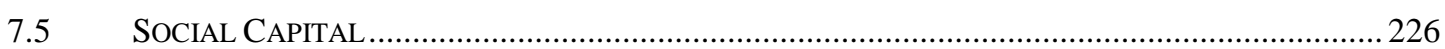

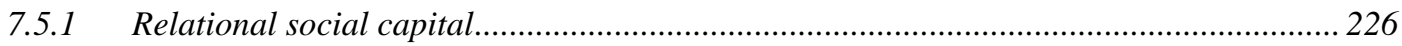

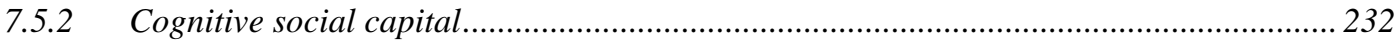

7.5.3 Structural social capital ............................................................................................ 236

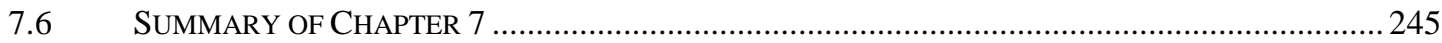

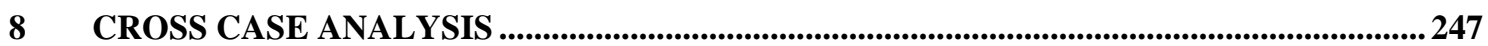

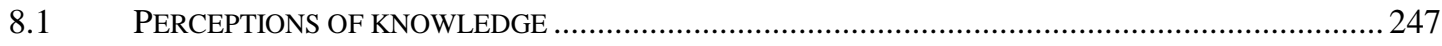

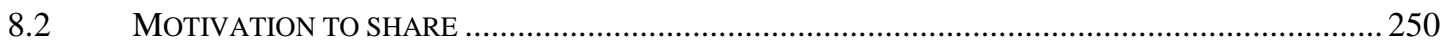

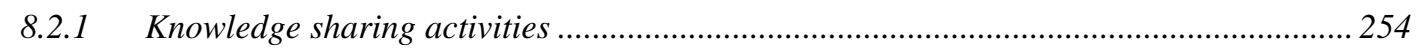

8.2.2 Choice of knowledge sharing channel.................................................................... 256

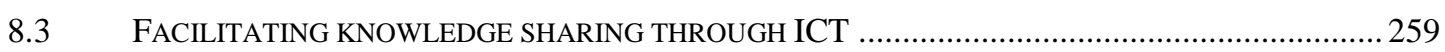

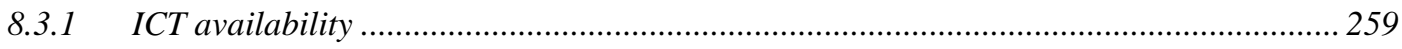




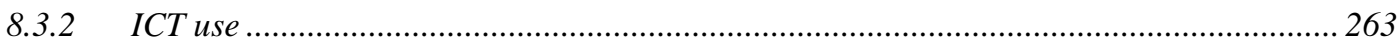

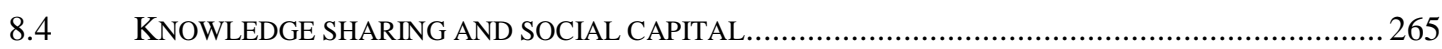

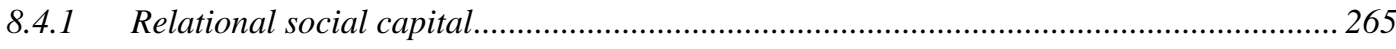

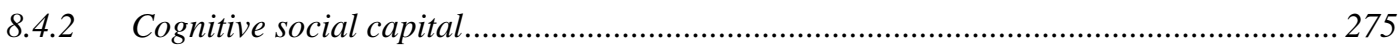

8.4.3 Structural social capital ........................................................................................... 279

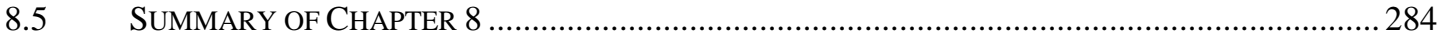

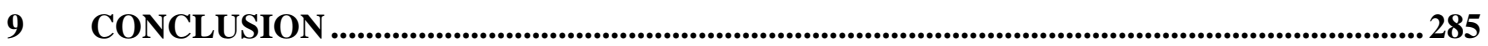

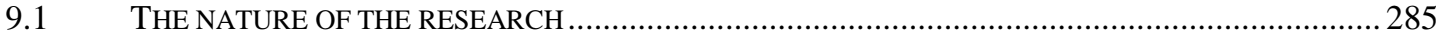

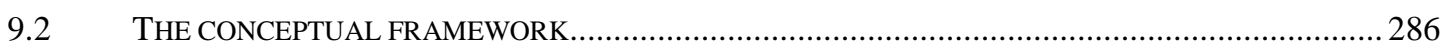

9.2.1 The nature of inter-organisational knowledge sharing in the public sector................... 288

9.2.2 Knowledge sharing and the role of social capital (SC).............................................. 292

9.2.3 Knowledge sharing and the role of ICT ........................................................................ 295

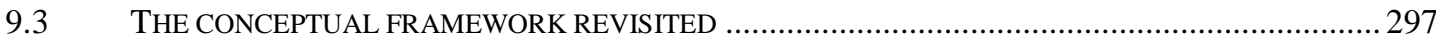

9.3.1 Collaboration design.................................................................................................. 299

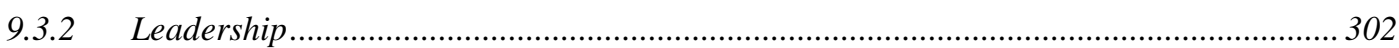

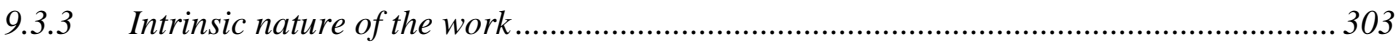

9.3.4 Perceived ease of use \& perceived usefulness of ICT tools............................................ 304

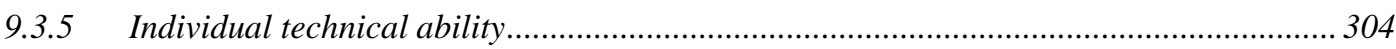

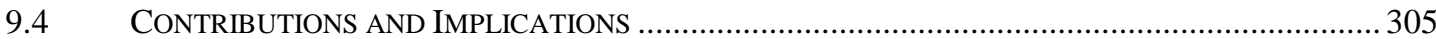

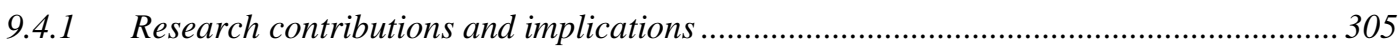

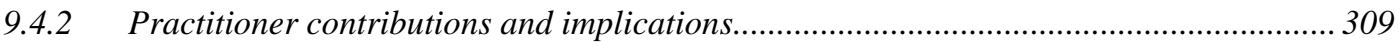

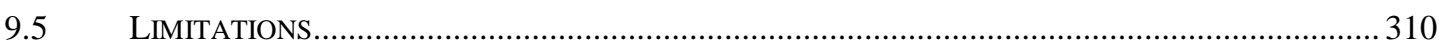

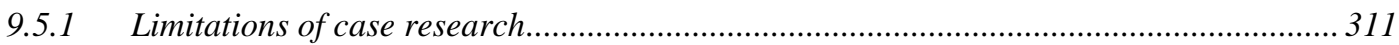

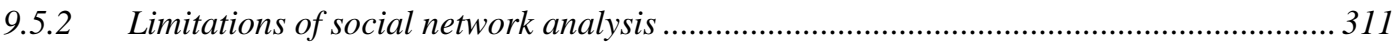

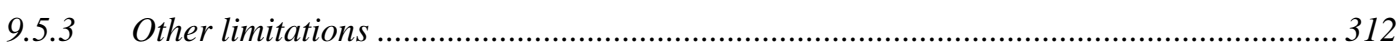

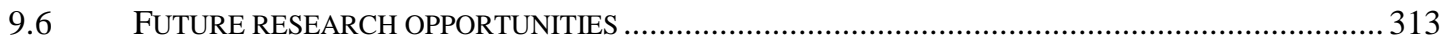

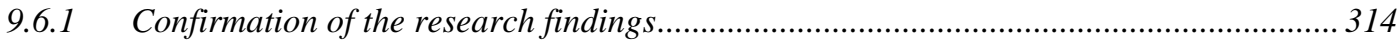

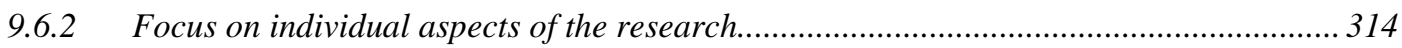

9.6.3 Application of the conceptual framework .................................................................. 315

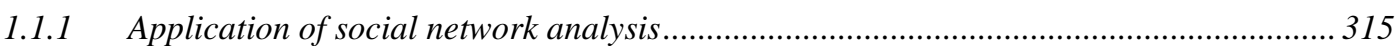

9.6.4 Extending the scope of the research ............................................................................. 315

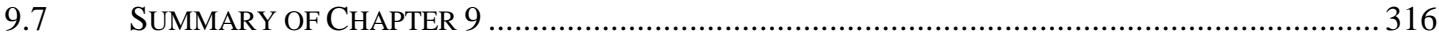

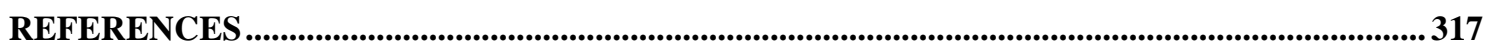

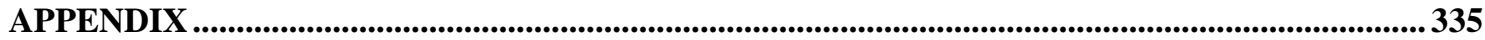




\section{List of Figures}

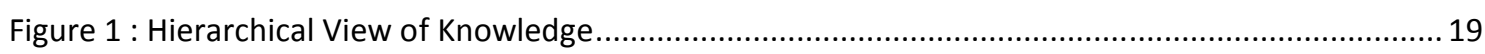

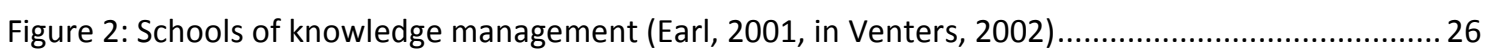

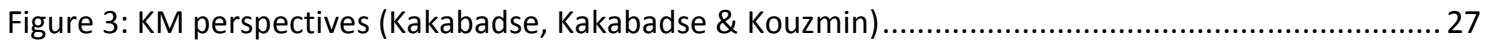

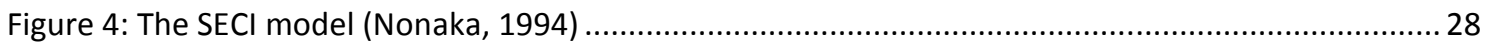

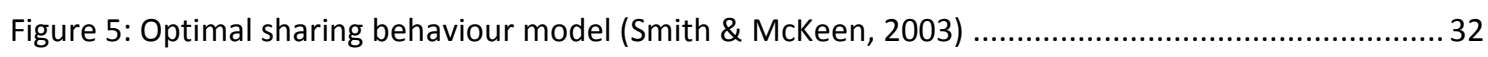

Figure 6 : Factors that influence knowledge sharing between individuals in organisations (Ipe, 2003) ... 34

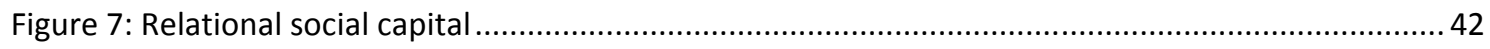

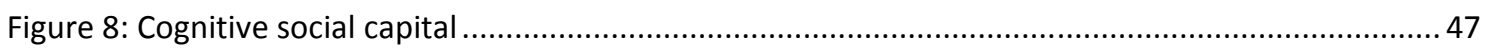

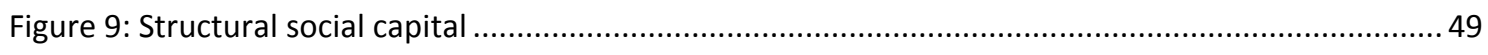

Figure 10: Examples of social capital measures (Widen-Wulff \& Ginman, 2004, p. 452) ........................53

Figure 11 : Conceptual model of information sharing (Miranda and Saunders, 2003) ..........................58

Figure 12 : Research model of the role of ICT in motivating knowledge sharing..................................59

Figure 13: Technology acceptance model (adapted from Davies, 1989 and Ventakash et al., 2001) .......59

Figure 14: Toolbox Vision of Shared Workspace (SSC, 2003) ............................................................... 71

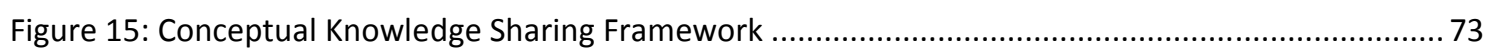

Figure 16: The four paradigms (adapted from Burrell \& Morgan, 1979) .............................................. 81

Figure 17: Paradigms: Language commonly associated with major research paradigms (adapted from

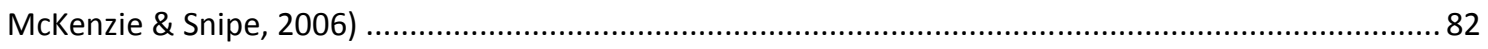

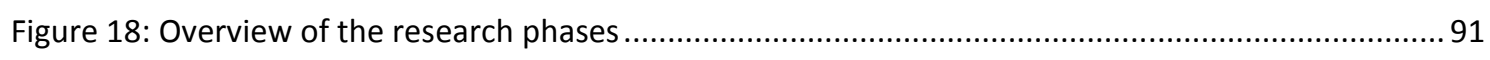

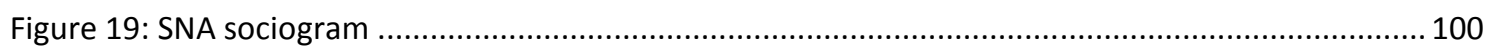

Figure 20: Research data collection \& analysis timeline ............................................................... 107

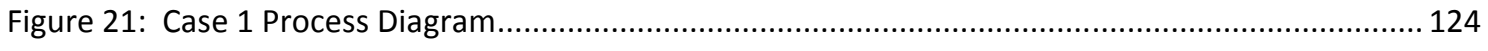

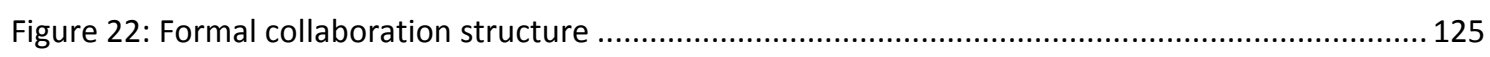

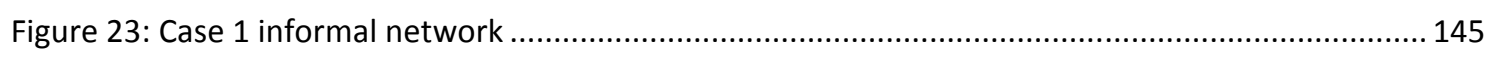

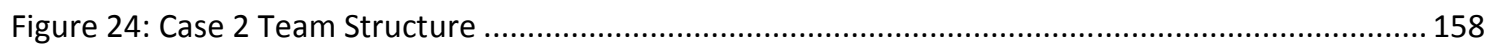

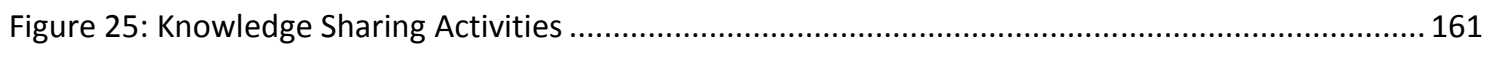

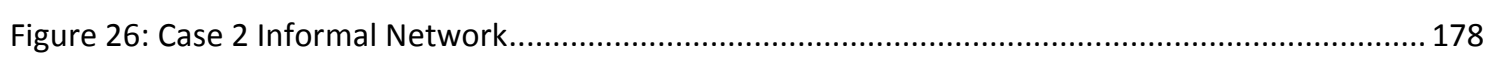

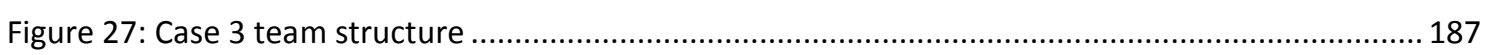

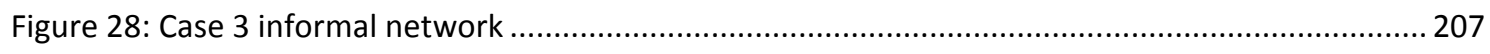

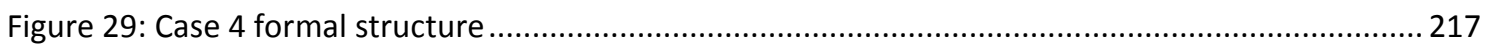

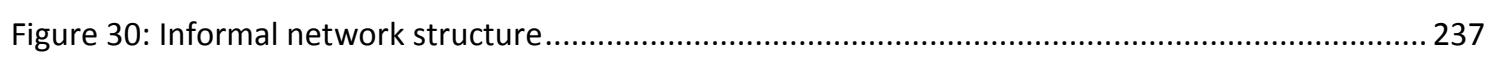

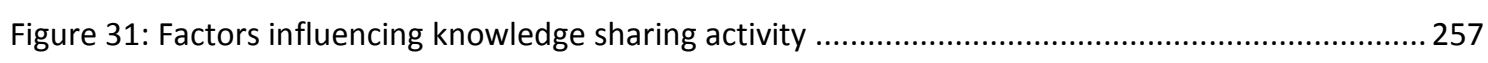

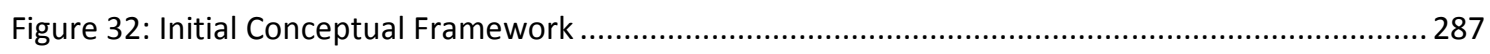

Figure 33: Context for inter-organisational knowledge sharing ................................................... 288

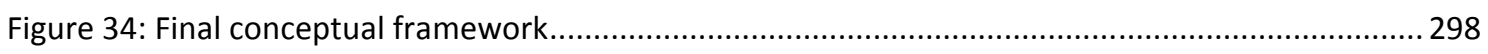




\section{List of Tables}

Table 1 : Key Characteristics of data, information and knowledge (adapted from Galliers and Newell, 2001)

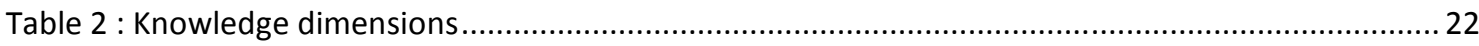

Table 3: Reasons preventing codification of tacit knowledge (adapted from Connell et al., 2003) .......... 23

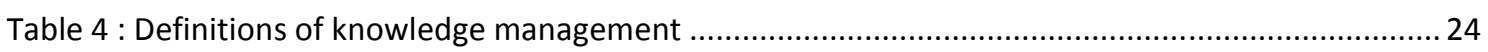

Table 5: Factors affecting knowledge sharing in an organisation (Lee \& Al-Hawamdeh, 2002) ................35

Table 6: Knowledge sharing activities (adapted from Jacobs \& Roodt, 2007) ..................................... 37

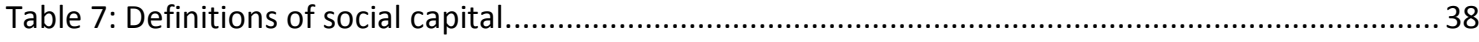

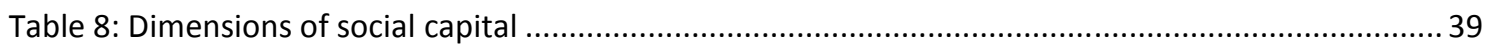

Table 9: Knowledge sharing studies focussing on social capital (adapted from Chow \& Chan, 2008) ......41

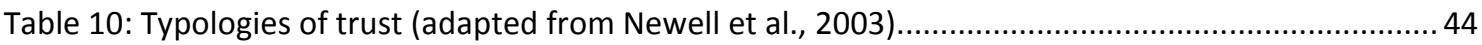

Table 11: Factors for successful collaboration (Pathfinder, 2001) .....................................................6 68

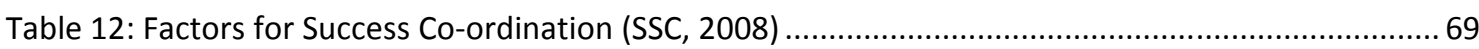

Table 13: Key characteristics of case research (Benbasat et al., 1987) ...............................................87

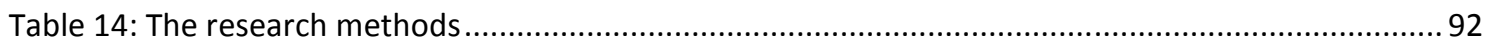

Table 15 : Criteria for assessing qualitative and quantitative research (Guba \& Lincoln, 1994) ..............93

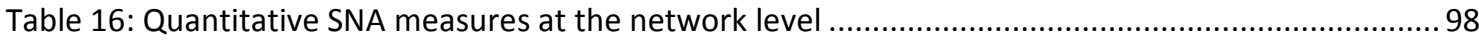

Table 17: Research of group structural properties (adapted from Pearce \& David, 1983)....................... 98

Table 18: Quantitative SNA measures at the individual level ........................................................... 100

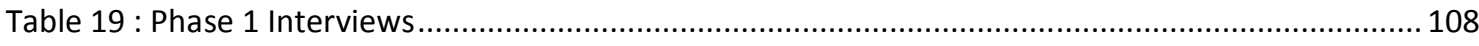

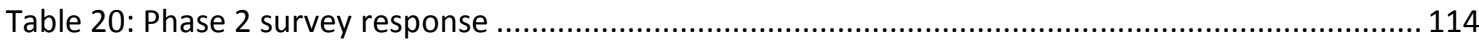

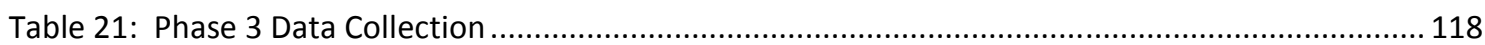

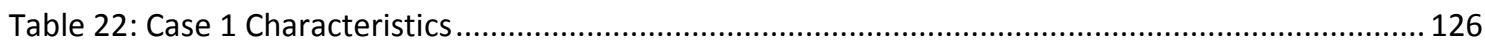

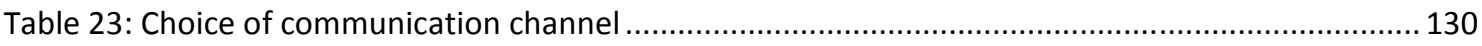

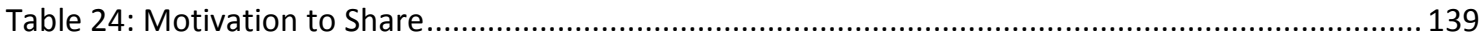

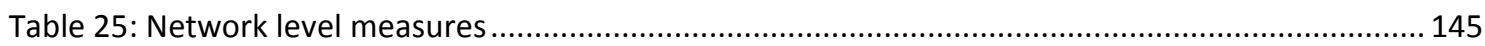

Table 26: Highest and lowest degree centrality scores............................................................... 147

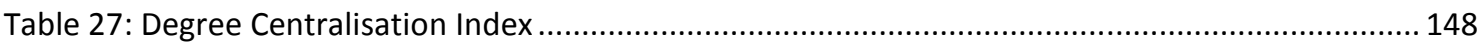

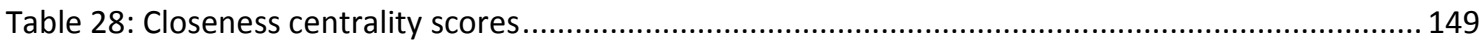

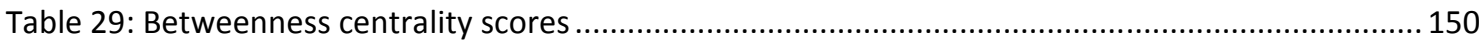

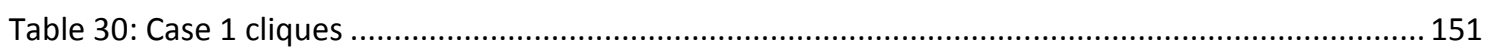

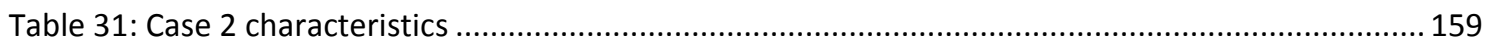

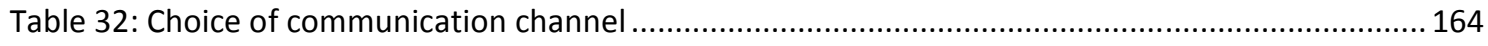

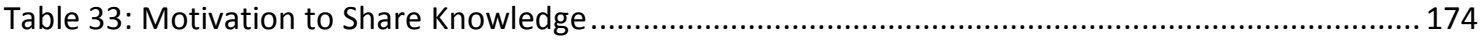

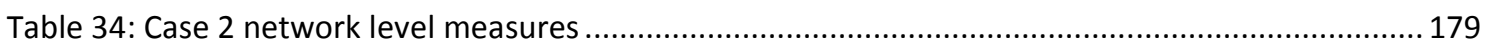

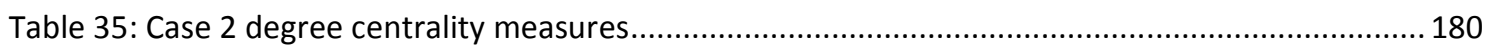


Table 36: Case 2 network centralisation index

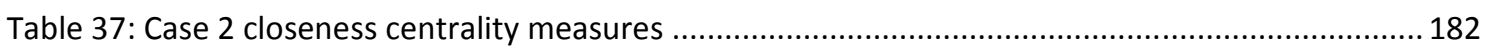

Table 38: Case 2 betweenness centrality measures ..................................................................... 183

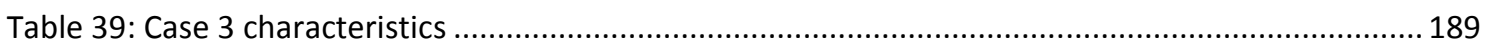

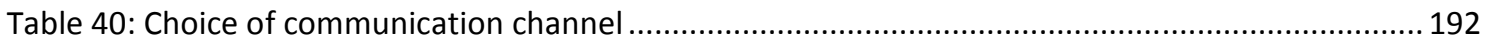

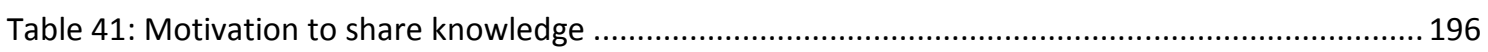

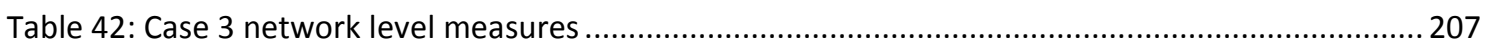

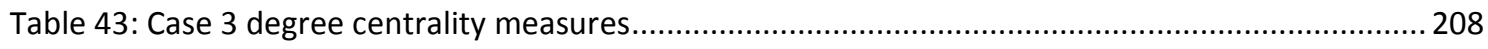

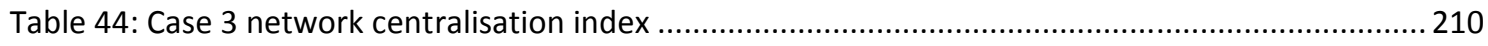

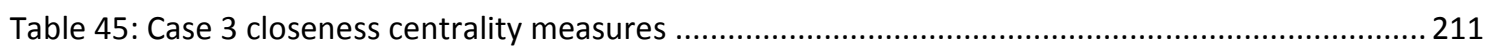

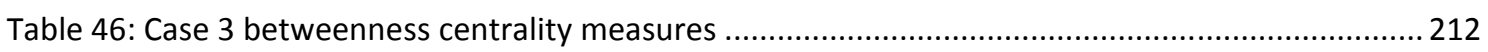

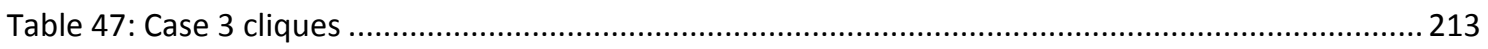

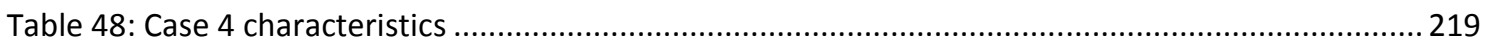

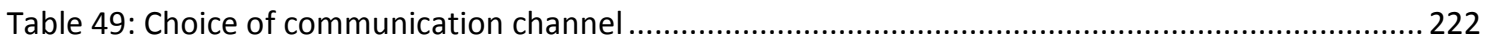

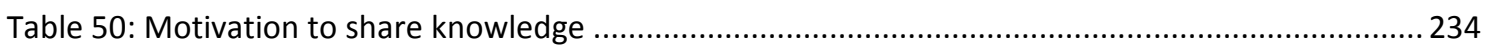

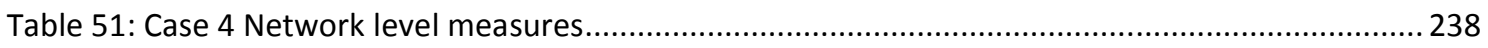

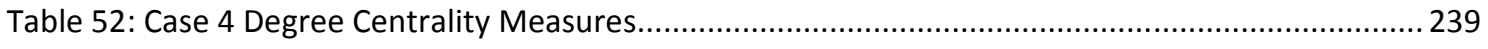

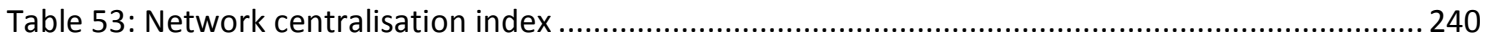

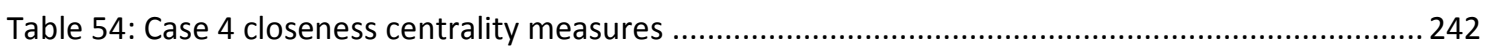

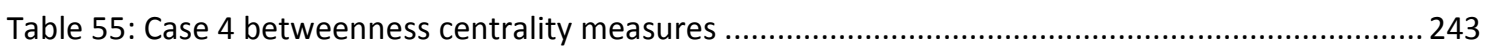

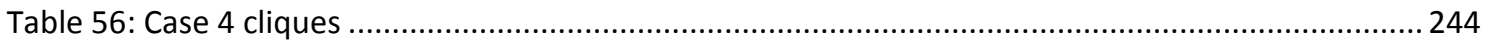

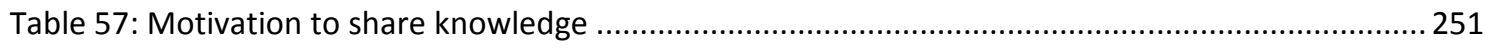

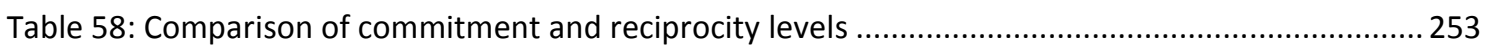

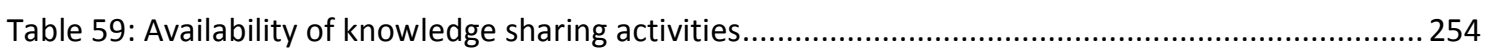

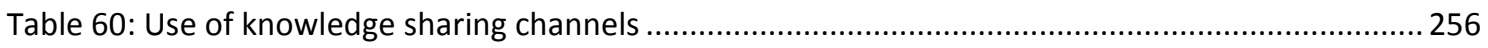

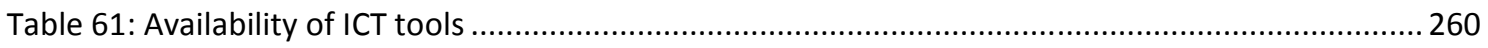

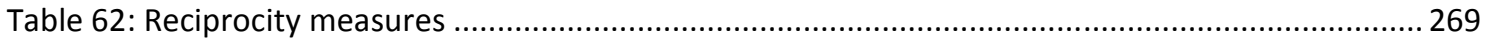

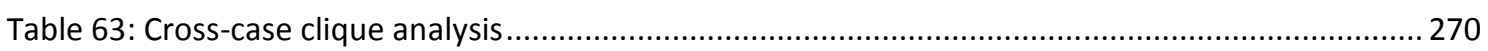

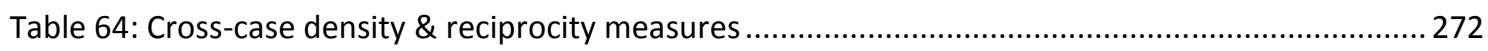

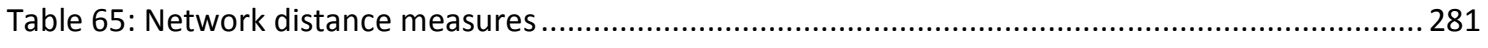

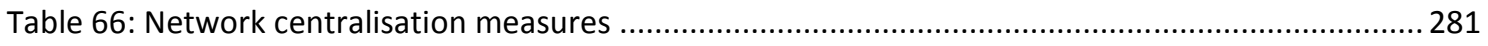

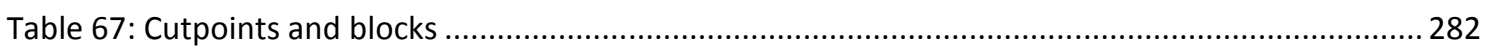




\section{INTRODUCTION}

Knowledge has been identified as an important strategic asset of organisations (Davenport \& Prusak, 1998; Bollinger \& Smith, 2001). The quest to create, maintain, and share that knowledge has spawned a wealth of literature over the past fifteen years and seen Knowledge Management (KM) evolve as both an established discipline and important business function (Argote, 1999; Wiig, 2003). KM presents an important and new approach to the issue of competitiveness and innovation and is undertaken by many types of organisations across many sectors (Newell, Robertson, Scarbrough \& Swan, 2002).

Knowledge sharing, an aspect of KM, deals with the ways in which knowledge may be shared between individuals, groups, or organisations (Connelly \& Kelloway, 2003). It is defined by Helmstadter (2003, p. 11-38) as "voluntary interactions between human actors [through] a framework of shared institutions, including law, ethical norms, behavioural regularities, customs, and so on”. As understanding of knowledge sharing has evolved, studies have refuted the reliance on technology and advocated an holistic approach that focuses more on people and less on ICT (Earl, 2001; Bartol \& Srivastava, 2002; McAdam \& McCreedy, 1999; Newell et al., 2002).

This study explores knowledge sharing in the context of inter-organisational collaboration within the public sector. It is an area that has received little research attention, yet is of increasing importance in light of the growth of collaborative endeavours between public sector organisations.

\subsection{The Nature of the Research Problem}

Advances in the sophistication and ubiquity of technology have enabled organisations to develop significant repositories of knowledge, yet research shows that technology has not been successful in enhancing and supporting knowledge sharing practices (Gold, Malhotra \& Segars, 2001; Huber, 2001; Ruggles, 1998). Knowledge sharing is now a core concern for organisations and is a major focus area for those involved with KM practices (Hendriks, 1999).

Early approaches to knowledge sharing focused on the use of ICT collaboration tools to promote and drive sharing behaviours. However, as understanding of the complexity 
of knowledge sharing has developed, the research focus has evolved to incorporate multiple perspectives including social, cultural, and philosophical approaches to understanding knowledge sharing (Rikowski, 2007).

Knowledge sharing research, particularly from non-technical perspectives, has been predominantly explored in the context of the private sector (Bate \& Robert, 2002; Taylor \& Wright, 2004). Initial analysis focused on how knowledge was shared between individuals but has expanded to consider knowledge sharing both within and across organisational teams. Growing interest in the benefits of inter-organisational collaboration has spawned a number of studies that explore knowledge sharing at an inter-organisational level. However, studies of this nature are still relatively few and there is a need to grow research in this area.

In the public sector, inter-organisational collaboration has manifested through the concept of Joined Up Government (JUG). This concept, first coined in 1997 by the United Kingdom (UK) government, "takes a holistic view; looking beyond institutional boundaries to the government's strategic objectives and seeks to establish the ethical, moral and legal base for policy" (Bullock, Mountford \& Stanley, 2001, p. 14). There must also be consideration of the appropriate management and organisational structures needed to deliver such cross-cutting objectives (Mulgan, 2005). JUG proposes a move away from public sector organisations operating as silos, towards a co-ordinated sector where knowledge sharing between organisations is the norm and is actively supported. Lips (2008) identified the benefits of JUG as the provision of better service to citizens; better coordination in government; more cost efficient work through sharing of resources and reduced duplication of effort and output; and innovation through new ways of working. Fundamentally, JUG requires public sector organisations to share information and knowledge across institutional boundaries. This requirement will increase as the occurrence of inter-organisational collaboration rises.

In New Zealand, Walker (2004) reported that the proliferation of inter-organisational collaboration in the government sector is increasing. Indeed, collaboration has received government attention through a range of initiatives including the State Services Commission (SSC) Review of the Centre (SSC, 2001); and The PathFinder Project (SSC, 2003), and most recently the development of New Zealand's evolving approach to JUG, which it defines as a system of world class State Services serving the 
government of the day and meeting the needs of New Zealanders (SSC, 2006). New Zealand's approach to JUG culminated in the identification and development of six key development goals. One of these goals, "Co-ordinated State Services", was defined as "New Zealand public sector organisations working together, sharing information, resources and responsibilities to achieve defined outcomes" (SSC, 2006). Thus, it is aimed specifically at supporting collaboration and sharing across the sector through a broad spectrum of approaches including ongoing partnerships between organisations, shared programmes of work, and discrete projects tasked with specific goals and finite timeframes. The predominant approach to the achievement of this goal advocates that public sector organisations focus on the use of ICT tools to facilitate knowledge sharing.

The public sector's technological approach is out of step with developments within the knowledge sharing literature. The most recent literature increasingly focuses on aspects of social capital (SC) as important considerations of sharing knowledge (Inkpen \& Tsang, 2005; Mu et al., 2008; Wu, Lin, Hsu \& Yeh, 2009). SC is defined as the networks, norms, trust, and mutual understanding that bind together members of human networks and communities, and enable participants to act together more effectively to pursue shared objectives (Huysman \& Wulf, 2002). The concept of SC encompasses a range of factors that have been studied in relation to knowledge sharing including trust (Luna-Reyes, Black, Cresswell, \& Pardo, 2008; Napahiet \& Ghoshal, 1998; Wu et al., 2007); shared purpose and goals (Cheng, Yeh \& Tu, 2008; Inkpen \& Tsang, 2005; Kim \& Lee, 2008), social networks (Hansen, 2002; Hoegl, Parboteeah \& Munson, 2003; Kim \& Lee, 2006; Kotlarsky \& Oshri, 2005). Though a common focus in the wider knowledge sharing literature, there is almost no research that explores the role of SC in the government context at either an organisational or inter-organisational level, and none that address these complex issues within the New Zealand context.

This study addresses three key gaps within the knowledge sharing research pertaining to the public sector. The first gap relates to a limited amount of research that explores knowledge sharing as a concept and practice within the public sector (Bate \& Robert, 2002; Bundred 2010; Jorgensen, 2004; Taylor \& Wright, 2004); the second gap concerns a lack of research that explores inter-organisational knowledge sharing in the sector (Mulgan, 2005; Tang, 2008); the third gap relates to the lack of understanding of 
knowledge sharing from a SC perspective (Gavigan, Ottitsch, \& Mahroum, 1999; Jorgensen, 2004; OECD, 2001; Takeuchi, 1998).

\subsection{The research questions}

The central theoretical aim of this research is to expand and deepen understanding of the dynamics of inter-organisational knowledge sharing. The research explores SC and ICT to determine the roles that each play in facilitating knowledge sharing, and seeks to identify potential relationships within and between these factors. Focussing on the New Zealand public sector, the empirical part to this study addresses the following research questions:

1. How is knowledge shared in inter-organisational collaborations in the public sector?

2. What is the role of SC in inter-organisational knowledge sharing in the public sector?

3. What is the role of ICT in inter-organisational knowledge sharing in the public sector?

\subsection{Research approach}

This study adopts a sequential mixed-methods approach, and employs multiple case study research. The research methods include one-on-one interviews and a survey incorporating social network analysis (SNA).

A mixed-methods approach offers a number of benefits. The triangulation of data sources can provide insight into different levels of units of analysis and can elaborate or expand the findings of one method against another (Creswell, 2003; Green, Caracelli \& Graham., 1989). In addition, findings that have been developed through a variety of methods or perspectives may also be more difficult to contest (Petter \& Gallivan, 2004), and can therefore help to address issues of validity and generalisability (Borkan, 2004).

Each case in the study was undertaken in three phases. The objective of Phase 1 was to gain a general understanding of the collaboration, its purpose, duration, formal team structure and to identify any formal processes in place. Phase 1 data were gathered 
through a number of one-on-one, semi-structured interviews with the senior team members from each participating agency.

Phase 2 data were gathered from all team members via a paper-based survey instrument. The survey design was based on the review of the literature and the findings from Phase 1. It combined social network, open and closed questions, and a number of questions relating to specific themes of the collaboration. The social network data collected through the surveys was analysed using UCINET@, a specialist network analysis software, and visualisation package NETDRAW@. Other data were analysed using SPSS 14.0@, a statistical analysis package.

The objective of the final research phase was to confirm and further mine the findings of Phases 1 and 2. Phase 3 data were gathered through a further round of one-on-one, semi-structured interviews with selected members of the teams. Interviewees from Phase 1 were excluded from this phase.

The outputs of the three research phases comprised rich descriptive data, nominal data, quantitative network data, and visual network maps.

As a final analysis step, cross-case analysis of all four cases was undertaken.

\subsection{Significance of this study}

Through the exploration of knowledge sharing practices in inter-organisational collaborative teams in the public sector, this study provides researchers and practitioners with theoretical and practical contributions to the field of knowledge sharing.

The findings from this study will augment the existing literature by addressing the three key gaps identified in Section 1.1. Firstly, the study will build on the limited research that explores the concept of knowledge sharing within the public sector; secondly, the study will contribute to knowledge about inter-organisational knowledge sharing in the sector; and, finally, the study will expand current understandings of inter-organisational sharing within the sector by focusing on SC.

In a practical sense, the primary intention of this study is to provide a better understanding of knowledge sharing in inter-organisational teams in the New Zealand public sector so that this can be used to inform planning and development of approaches 
to assist inter-organisational knowledge sharing efforts. Through a better understanding of the issues, resourcing and funding can be appropriately allocated to assist individuals engaged in collaborative initiatives. This is particularly important in an environment where the occurrence of inter-organisational collaboration is increasing. The study will also benefit private organisations engaged in collaborative endeavours with public sector organisations.

It is hoped that the findings will also benefit other types of collaboration, such as government collaboration with private sector organisations, as well as collaboration between private sector organisations. Although there are differences between organisations within these sectors, much of the research is likely to be relevant and applicable across both private and public domains.

\subsection{Structure of the thesis}

This chapter provides the starting point for the study. It introduces the research problem, presents the research questions, and discusses the significance of the study and its practical and academic contributions. Chapter 2 presents the literature review and conceptual framework developed for this study. Chapter 3 describes the research process, and justifies the researcher's worldview and choice of research design and methods. It also details the data collection and analysis used in the study. Chapters $4-$ 7 present a detailed account of each of the four research Cases. A cross-analysis of the cases is then presented in Chapter 8. Chapter 9 concludes the research by outlining the main findings of the research and incorporating these into the conceptual framework. The chapter also discusses the significance, implications and limitations of my findings, suggesting areas for future research.

\subsection{Terminology}

This section lists a specialist terms pertaining the specific areas of focus in the thesis.

Knowledge Management: The approaches, mechanisms, and processes used to organisations to create, retain, and share knowledge.

Knowledge Sharing: the voluntary interactions between human actors [through] a framework of shared institutions, including law, ethical norms, behavioural regularities, 
customs and so on ... the subject matter of the interactions between the participating actors is knowledge. Such an interaction itself may be called sharing of knowledge" (Helmstadter, 2003, p.11).

Social Capital: The networks, norms, trust and mutual understanding that bind together members of human networks and communities, and enable participants to act together more effectively to pursue shared objectives (Huysman \& Wulf, 2003).

Social Network Analysis: A research method used to visualise and measure interactions between individuals and groups. 


\section{LITERATURE REVIEW AND CONCEPTUAL FRAMEWORK}

\subsection{Introduction}

The purpose of the literature review is to assess the existing knowledge on the research topic, confirm the tentative belief of the need for research in this area, and to refine the proposed research questions. Although the review was completed prior to analysis and discussion of the research findings, the researcher has maintained a continuous watch on emerging literature and the review has been constantly updated to incorporate new and relevant studies.

The literature review is presented in six main sections:

- Section 1: Introduces the concept of knowledge, from its epistemological foundations through its importance to today's organisations.

- Section 2: Presents a selection of definitions, frameworks, and key issues relating to knowledge sharing - including knowledge sharing channels and individuals' motivation to share.

- Section 3: Introduces the concept of SC and associated frameworks, discusses the relational, cognitive and structural dimensions of SC and social networks.

- Section 4: Describes how ICT has been used to support knowledge sharing and outlines the differing viewpoints of scholars in relation to this use. The section also discusses the relationship between ICT and types of knowledge, and the way in which ICT has been used as a communication channel.

- Section 5: Introduces the public sector as context for the study. It provides a brief history of the sector and its evolution, discusses inter-organisational collaboration and the way in which knowledge sharing underpins that collaboration. The section concludes by detailing current approaches to knowledge sharing in the sector and summarises the limited research in this area.

- Section 6: This section presents the conceptual framework developed for use in this study and the development of the research questions. The framework 
provides the boundaries for the research; identifies the key areas of focus; and posits potential relationships between these elements.

\subsection{Knowledge and its Management}

\subsubsection{Epistemological foundations of knowledge}

The philosophical underpinnings of knowledge can be traced back to the time of the Ancient Greeks, when Plato determined that knowledge occurs when true belief is accompanied by rationale account. Simply, it is not enough to believe that something is true; the belief must be accompanied by propositions or statements that justify the certainty of the belief. In turn, Aristotle contested the element of certainty and proposed that while some objects of human knowledge, such as mathematics, allow for certain knowledge, others, such as ethics and politics, can only allow for probable knowledge due to the number of variables involved. These early Greek philosophers provided the foundation for centuries of debate for subsequent scholars.

Today, knowledge has become a commodity that is highly valued by organisations, and the ability to acquire and utilise knowledge quickly is seen as the single means by which organisations can sustain competitive advantage (Winter, 1995). Without knowledge, organisations would be unable to effectively make use of materials, process, and financial capital (Davenport \& Prusak, 1998).

\subsubsection{Knowledge definitions}

One of the most common and elementary discussions within the KM literature concerns the distinction between data, information, knowledge, and, in some instances, wisdom (Gurteen, 1998). This is commonly referred to as the hierarchical view (see Figure 1).

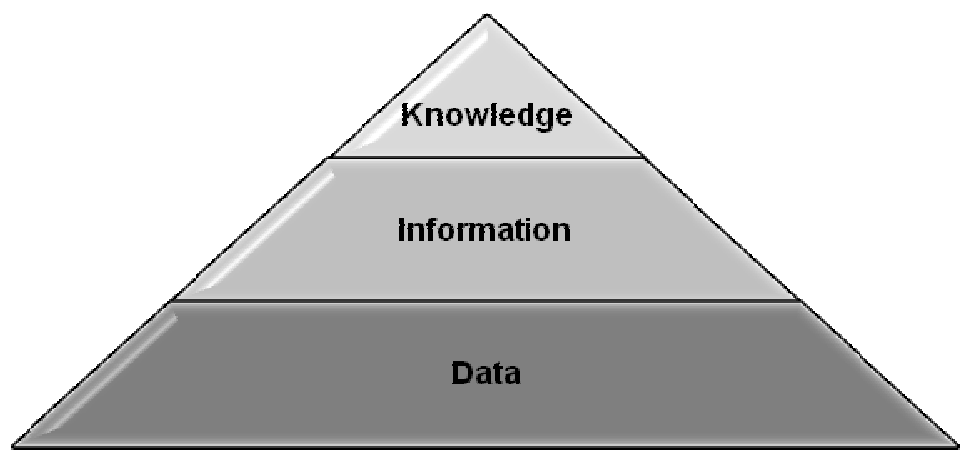

Figure 1 : Hierarchical View of Knowledge 
In this scenario, data is commonly agreed upon as relating to raw numbers and/or facts, information is data that has been refined or processed, and knowledge is authenticated information (Alavi \& Leidner, 2001). Data is viewed as the first step in the knowledge pyramid, from which an upward transition is made in order for data to be transformed into knowledge. Tuomi (1999) contests this view and asserts that the pathway from data to knowledge is, in fact, the reverse. The crux of Tuomi's argument lies in the belief that knowledge is the result of cognitive processing triggered by an influx of new stimuli (Alavi \& Leidner, 2001). Regardless of the directional nature of the knowledge hierarchy, understanding the connection between the individual elements is considered essential to undertaking knowledge work successfully (Davenport \& Prusak, 1998).

The knowledge pyramid has also been extended by some researchers to encompass wisdom (see Courtney, Haynes \& Paradice, 2005; Rooney \& McKenna, 2005). Though outside the scope of this thesis, within the extant literature wisdom is acknowledged as a growing area of interest.

Table 1 documents other key differences between data, information, and knowledge as noted by Galliers \& Newell (2001).

Table 1 : Key Characteristics of data, information and knowledge (adapted from Galliers and Newell, 2001)

\begin{tabular}{|l|l|l|}
\hline Data & Information & Knowledge \\
\hline Explicit & Interpreted & Tacit/embedded \\
\hline Exploit & Explore & Create \\
\hline Use & Build/construct & Rebuild/reconstruct \\
\hline Accept & Confirm & Disconfirm \\
\hline Follow old recipes & Amend old recipes & Develop new recipes \\
\hline Nolearning & Single-loop learning & Double-loop learning \\
\hline Direction & Communication & Sense-making \\
\hline Prescriptive & Adaptive & Seminal \\
\hline Efficiency & Effectiveness & Innovation/redundancy \\
\hline Predetermined & Constrained & Flexible \\
\hline $\begin{array}{l}\text { Technical } \\
\text { systems/networks }\end{array}$ & Socio-technical systems & Social networks \\
\hline Context-free & Outer context & Inner Context \\
\hline
\end{tabular}

Three interesting points identified by Galliers and Newell (2001) can be seen in Table 1. The first point relates to the importance of context. Data are discerned as context-free and as such do not facilitate learning or any requirement for sense-making. However, information, while still lacking the richness of knowledge, provides an outer context and structure that sets it apart from data. The second point of note is the association between knowledge and sense-making, whereby we take action because of the sense we 
make of a situation through our own knowledge. So action and knowledge are brought together through our individual notion of sense-making (Weick, 1995). Thirdly, Galliers \& Newell (2001) distinguish between the types of systems in which data, information, and knowledge are likely to occur. Data are commonly a characteristic of technical systems, information is more commonly a feature of socio-technical systems, while knowledge is a characteristic of social systems.

Seminal KM authors Nonaka \& Takeuchi (1995) define knowledge as a "dynamic human process of justifying personal belief toward the 'truth'" (p. 58). They contend that knowledge enables an entity's capacity for effective action to be increased. While this definition harks back to Plato's requirement for justification, it also adds a further dimension to the way in which knowledge can increase the application of effective action. Davenport and Prusak (1998) define knowledge as "a fluid mix of framed experience, values, contextual information, and expert insight that provides a framework for evaluating and incorporating new experiences and information" (p. 5). This definition supports Galliers and Newell's (2001) belief about the dynamic and contextual nature of knowledge.

\subsubsection{Knowledge typologies}

Scholars have categorised knowledge into a range of dimensions in an attempt to better understand the full scope of the phenomenon (see Table 2).

A commonly discussed perspective categorises knowledge as either explicit or tacit. Explicit knowledge is defined as "transmittable in formal, systematic language" (Nonaka, 1994, p. 16). It contains information that has been codified into a format that others may readily understand and use, is easily articulated, and can be readily stored in some tangible format. In an organisational context, this knowledge may be captured and stored within an instruction manual, a set of processes or procedures, or within a technology system such as a database.

Tacit knowledge is subjective and experience-based, such that it cannot easily be expressed in words, but also includes cognitive skills such as beliefs, images, intuition, and mental models as well as technical skills such as craft and know-how (Nonaka \& Takeuchi, 1995). Early work in the domain of tacit knowledge was carried out by Polanyi (1967), who suggested that we know more than we can tell, simply because 
tacit knowledge is not easily articulated. In an organisational context, tacit knowledge has been defined as consisting of the collective mindsets of everyone in the organisation (Saint Onge, 1996).

Table 2 : Knowledge dimensions

\begin{tabular}{|c|c|c|}
\hline Knowledge Dimensions & Description & References \\
\hline Explicit and tacit & $\begin{array}{l}\text { Explicit knowledge is expressed formally, and } \\
\text { can be easily communicated or shared. } \\
\text { Tocit knowledge is experiential and } \\
\text { contextualised, and is difficult, if not } \\
\text { impossible, to codify. }\end{array}$ & $\begin{array}{l}\text { Polanyi, 1966; } \\
\text { Brown \& Duguid, 1998; } \\
\text { Nonaka \& Takeuchi, } 1995 .\end{array}$ \\
\hline $\begin{array}{l}\text { Component and } \\
\text { architectural }\end{array}$ & $\begin{array}{l}\text { Component knowledge relates to distinct } \\
\text { aspects of an organisation's operations and } \\
\text { may be held individually or collectively. } \\
\text { Architecturol knowledge relates to organisation } \\
\text { wide routines for co-ordinating components. }\end{array}$ & Henderson \& Clark, 1990 \\
\hline $\begin{array}{l}\text { Embrained, embodied, } \\
\text { encultured, embedded, } \\
\text { and encoded }\end{array}$ & $\begin{array}{l}\text { Embroined knowledge is dependent on } \\
\text { conceptual skills and cognitive ability. } \\
\text { Embodied knowledge is action oriented and } \\
\text { likely to be only partly explicit. } \\
\text { Encultured knowledge refers to the process of } \\
\text { achieved shared understandings. } \\
\text { Embedded knowledge resides in systematic } \\
\text { routines. } \\
\text { Encoded knowledge is conveyed by signs and } \\
\text { symbols in artefacts such as books and } \\
\text { manuals. }\end{array}$ & Blackler, 1995 \\
\hline Individual and collective & $\begin{array}{l}\text { Individual knowledge held by an individual. } \\
\text { Collective knowledge held by more than one } \\
\text { individual, ie by an organisation. }\end{array}$ & $\begin{array}{l}\text { Spender, } 1996 \\
\text { Chua, } 2001\end{array}$ \\
\hline Cultural & $\begin{array}{l}\text { Cultural knowledge is based on shared beliefs } \\
\text { and understandings. }\end{array}$ & Choo, 1998 \\
\hline Private and public & $\begin{array}{l}\text { Privte knowledge relates to specific } \\
\text { organisational knowledge. } \\
\text { Public knowledge is accessible from the public } \\
\text { domain. }\end{array}$ & Matusik \& Hill, 1998 \\
\hline $\begin{array}{l}\text { Personalisation and } \\
\text { codification }\end{array}$ & $\begin{array}{l}\text { Personolised knowledge is closely tied to the } \\
\text { person who developed it and shared mainly } \\
\text { through direct person-to-person contacts. } \\
\text { codified knowledge is codified and stored in } \\
\text { databases and documents. }\end{array}$ & Hansen et al., 1999 \\
\hline Migratory & Shared knowledge can move & Badaracco [1991] \\
\hline $\begin{array}{l}\text { Experiential, reported, } \\
\text { intimate, and declared }\end{array}$ & $\begin{array}{l}\text { Experientiol knowledge is pragmatic and } \\
\text { practical } \\
\text { Reported knowledge is published or disclosed } \\
\text { Intimote knowledge is deep seated or } \\
\text { experienced } \\
\text { Declared knowledge is professed or purported }\end{array}$ & Wikstrom et al.. [1994] \\
\hline
\end{tabular}

Hedesstrom \& Whitley (2000) present two perspectives on tacit knowledge; the "difficulty" and the "de facto" perspectives. These represent two opposing viewpoints; one that advocates tacit knowledge as being "difficult" but possible to share, the other viewpoint posits that tacit knowledge simply cannot be codified and therefore cannot be shared. In this latter perspective, the difficulty approach can be understood through the example of asking an individual to describe a routine task they do every day, for example riding a bike. An articulated response is difficult to give and difficult for the 
receiver to understand or interpret. The easier solution is for the rider to "show" how it is done. This is described by Sutton (2001, p. 82) as "the natural unconscious execution of a task and the ability to talk about a task". Indeed, Stenmark (2001, p. 10) asserts that "expertise is a quality highly dependent on tacit knowledge, and it can often only be observed and recognized through its resulting actions". Connell, Klein \& Powell (2003) confirm their support for Hedesstrom \& Whitley's de facto perspective of tacit knowledge and conclude that tacit knowledge cannot be codified. Connell et al. (2003) identify five reasons for this conclusion, as summarised in Table 3.

Table 3: Reasons preventing codification of tacit knowledge (adapted from Connell et al., 2003)

\begin{tabular}{|l|l|}
\hline Reason & Description \\
\hline Inefficiency & $\begin{array}{l}\text { The effort required to extract and codify tacit knowledge may outweigh the } \\
\text { potential returns }\end{array}$ \\
\hline Technology & $\begin{array}{l}\text { Issues include the speed of processing and the ability to capture the } \\
\text { essence of the knowledge }\end{array}$ \\
\hline Motivation & $\begin{array}{l}\text { You can lead a person to knowledge but you can't make them internalise it } \\
\text { (Choo, 1998) }\end{array}$ \\
\hline $\begin{array}{l}\text { Language } \\
\text { Internalising and } \\
\text { externalising } \\
\text { knowledge }\end{array}$ & $\begin{array}{l}\text { Problems associated with coding, communication, and decoding knowledge } \\
\text { wide range of recipients }\end{array}$ \\
\hline
\end{tabular}

The Personalisation - Codification dimension is also one that is more widely understood in the organisational context. Hansen, Nohria \& Tierney (1999) depict personalised knowledge as being closely tied to the person who developed it, and suggest that it is shared mainly through person-to-person contact. Conversely, codified knowledge is more explicit in nature and is commonly found within organisational information repositories.

\subsubsection{Knowledge and the organisation}

Knowledge has been recognised as a valuable strategic asset within organisations (Zack, 2000). This realisation has initiated a strategy shift within organisations, moving away from traditional theories to one in which a knowledge-based view takes hold (Ruggles \& Holtshouse, 1999). The knowledge-based theory of the firm has its roots in the strategic management literature, and has been driven by the advent of the knowledgebased economy. The theory propounds that knowledge-based resources are generally difficult to imitate because they are created and held by individuals and are thus socially complex (Grant, 2002). It is this complexity that differentiates knowledge from other organisational resources (Spender, 1996) and has seen KM heralded as an important and 
new approach to the issues of competitiveness and innovation, and it is undertaken by many types of organisations across many sectors (Newell et al., 2002).

\subsubsection{Defining knowledge management}

Despite many attempts to define KM, scholars have failed to identify a universally accepted definition (see Table 4). A key similarity between many definitions is the reference to the four generic knowledge processes of the creation, storage and retrieval, sharing, and application of knowledge (Alavi \& Leidner, 2001). The descriptive language used to describe these processes can differ between definitions, but describe the same processes.

Table 4 : Definitions of knowledge management

\begin{tabular}{|l|l|}
\hline Definition & Author \\
\hline $\begin{array}{l}\text { Distinct but interdependent processes of } \\
\text { knowledge creation, knowledge storage and } \\
\text { retrieval, knowledge transfer, and knowledge } \\
\text { application }\end{array}$ & Alavi \& Leidner, 2001 \\
\hline $\begin{array}{l}\text { How organisations create, retain, and share } \\
\text { knowledge }\end{array}$ & Argote, 1999; Huber, 1991 \\
\hline $\begin{array}{l}\text { The tools, techniques, and strategies to retain, } \\
\text { analyse, organise, improve, and share business } \\
\text { expertise }\end{array}$ & Groff \& Jones, 2003 \\
\hline $\begin{array}{l}\text { The identification, optimisation, and active } \\
\text { management of intellectual assets, either in the } \\
\text { formof explicit knowledge held in artefacts or as } \\
\text { tacit knowledge possessed by individuals or } \\
\text { communities }\end{array}$ & \\
\hline $\begin{array}{l}\text { The collection of processes that govern the } \\
\text { creation, dissemination and utilisation of } \\
\text { knowledge to fulfil organisational objectives }\end{array}$ & Murray \& Myers, 1997 \\
\hline $\begin{array}{l}\text { KM is the explicit control and management of } \\
\text { knowledge within an organisation aimed at } \\
\text { achieving the company's objectives }\end{array}$ & van der Spek \& Spijkervet, 1997 \\
\hline $\begin{array}{l}\text { KM is any process or practice of creating, } \\
\text { acquiring, capturing, sharing, and using } \\
\text { knowledge, wherever it resides, to enhance } \\
\text { learning and performance in organisations }\end{array}$ & Swan et al., 1999 \\
\hline
\end{tabular}

Snowden (1999) augments the process perspective with reference to distinct knowledge types, and the introduction of the concept of multi-level knowledge ownership through the consideration of individual and communities. While Argote (1999) and Huber (1991) encapsulate KM processes in their context of the organisation, other definitions specifically connect KM processes with the achievement of organisational objectives (Murray \& Myers, 1997) or to organisational learning and performance (Swan, Newell, 
Scarbrough \& Hislop, 1999). Other definitions also refer to the tools, the techniques, and the strategies by which KM can be enacted (Groff \& Jones, 2003).

\subsubsection{Approaches to knowledge management}

Early approaches to KM emphasised a technical perspective (Alavi \& Leidner, 2001). In this approach, initiatives focused heavily on codification and storage of knowledge which were largely approached through the use of ICT-based solutions. In many instances, ICT has been found to be effective in the facilitation of data and information transmission but not able to replace the richness of communication, interactivity and learning that comes with dialogue (Fahey \& Prusak, 1998). Ruggles (1998) stated that "if technology solves your problem, yours was not a knowledge problem" (p. 88). As an understanding of $\mathrm{KM}$ evolved, scholars advocated an integrated approach that addressed organisational, cultural, and technical infrastructures as the most effective approach (Gold, Malhotra \& Segars, 2001; Grover \& Davenport, 2001). This view redefined the role of ICT as one which positioned it as an enabler of KM, rather than a driver (Martiny, 1998). Therefore IT tools such as yellow pages and knowledge directories could be considered a complement to an organisation's knowledge sharing activities, but would not determine a positive knowledge sharing culture (Connelly \& Kelloway, 2003). In order to enable more effective management of knowledge the next generation of KM systems must incorporate the structural, shared cognitive, and relational dimensions of an organisation. (Ackerman \& Halverson, 2004).

Earl (2001) encapsulates KM approaches into a three dimensional framework: the Technocratic, based on information and management technologies; the Economic, based on the management and exploitation of organisations assets for financial gain, and; the Behavioural, within which the organisational and spatial attributes consider the element of social interaction (see Figure 2). 


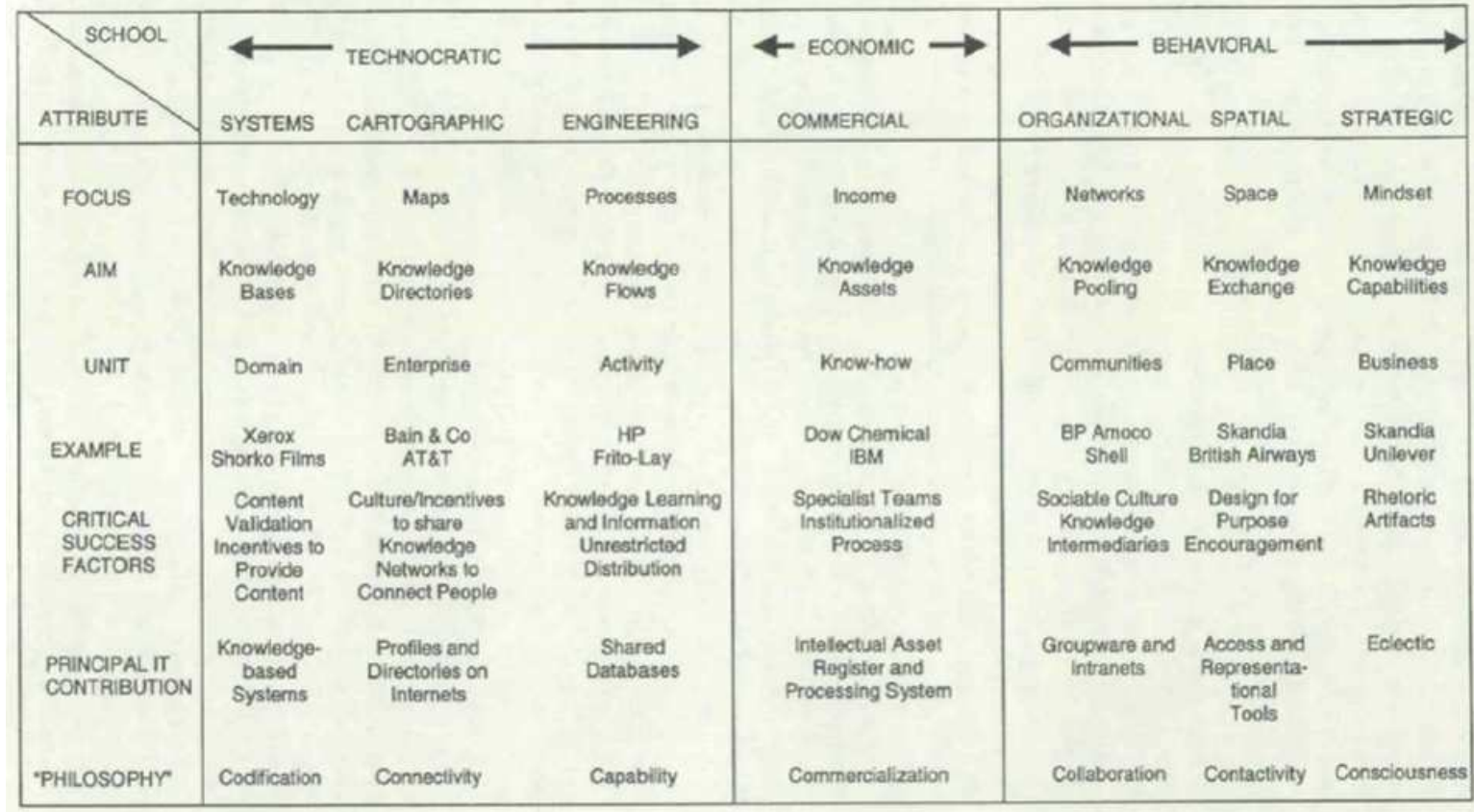

Figure 2: Schools of knowledge management (Earl, 2001, in Venters, 2002)

McAdam and McCreedy (1999) propose an alternative framework identifying three KM dimensions. In the intellectual capital dimension, the approach is mechanistic in nature and assumes that knowledge can be treated as an asset. The knowledge categories dimension positions knowledge as discrete elements and involves the transformation of knowledge through socialisation processes. The social constructionist dimension assumes a wide definition of knowledge that is regarded as intrinsically linked to the social and learning processes of an organisation.

Other models proposed by Swan \& Newell (2000), Murray (2000) and Tisen et al. (2000) are detailed in a summary of models presented by Kakabadse, Kakabadse and Kouzmin (2003) (see Figure 3). The network model is strategically intended to tap across organisational levels. From this perspective, individuals' actions are perceived as being influenced by the networks of relationships in which they are embedded (Newell \& Swan, 2000) and it is assumed that the motives of individuals are social as well as economic (Newell et al., 2002). 


\begin{tabular}{|c|c|c|c|c|c|}
\hline & $\begin{array}{l}\text { Philosophy-based } \\
\text { model }\end{array}$ & Cognitive model & Network model & Community model & Quantum model \\
\hline $\begin{array}{l}\text { Treatment of } \\
\text { knowledge }\end{array}$ & $\begin{array}{l}\text { Knowledge is } \\
\text { "justified true belief" }\end{array}$ & $\begin{array}{l}\text { Knowledge is } \\
\text { objectively defined } \\
\text { and codified as } \\
\text { concepts and facts }\end{array}$ & $\begin{array}{l}\text { Knowledge is external } \\
\text { to the adopter in } \\
\text { explicit and implicit } \\
\text { forms }\end{array}$ & $\begin{array}{l}\text { Knowledge is } \\
\text { constructed socially } \\
\text { and based on } \\
\text { experience }\end{array}$ & $\begin{array}{l}\text { System of } \\
\text { possibilities }\end{array}$ \\
\hline $\begin{array}{l}\text { Dominant } \\
\text { metaphor }\end{array}$ & Epistemology & Memory & Network & Community & Paradox \\
\hline Focus & Ways of knowing & $\begin{array}{l}\text { Knowledge capture } \\
\text { and storage }\end{array}$ & $\begin{array}{l}\text { Knowledge } \\
\text { acquisition }\end{array}$ & $\begin{array}{l}\text { Knowledge creation } \\
\text { and application }\end{array}$ & $\begin{array}{l}\text { Solving paradox and } \\
\text { complex issues }\end{array}$ \\
\hline Primary aim & Emancipation & $\begin{array}{l}\text { To codify and capture } \\
\text { explicit knowledge } \\
\text { and information - } \\
\text { knowledge } \\
\text { exploitation }\end{array}$ & $\begin{array}{l}\text { Competitive } \\
\text { advantage }\end{array}$ & $\begin{array}{l}\text { Promote knowledge } \\
\text { sharing }\end{array}$ & Learning systems \\
\hline Critical lever & $\begin{array}{l}\text { Questioning, reflecting } \\
\text { and debating }\end{array}$ & Technology & Boundary spanning & $\begin{array}{l}\text { Commitment and } \\
\text { trust }\end{array}$ & Technology \\
\hline $\begin{array}{l}\text { Primary } \\
\text { outcomes }\end{array}$ & New knowledge & $\begin{array}{l}\text { Standardization, } \\
\text { routinization and } \\
\text { recycling of } \\
\text { knowledge }\end{array}$ & $\begin{array}{l}\text { Awareness of external } \\
\text { development }\end{array}$ & $\begin{array}{l}\text { Application of new } \\
\text { knowledge }\end{array}$ & $\begin{array}{l}\text { Creation of multi- } \\
\text { reality }\end{array}$ \\
\hline $\begin{array}{l}\text { Role of IT } \\
\text { based tools }\end{array}$ & Almost irrelevant & $\begin{array}{l}\text { Critical integrative } \\
\text { mechanism }\end{array}$ & $\begin{array}{l}\text { Complimentary } \\
\text { interactive mechanism }\end{array}$ & $\begin{array}{l}\text { Supporting } \\
\text { integrative } \\
\text { mechanism }\end{array}$ & $\begin{array}{l}\text { Critical-Knowledge } \\
\text { centric }\end{array}$ \\
\hline
\end{tabular}

Figure 3: KM perspectives (Kakabadse, Kakabadse \& Kouzmin)

The personalisation and codification approach to knowledge sharing proposed by Hansen et al. (1999) reflects the human and technological aspects of knowledge. The codification approach sees organisations rely heavily on the storage of knowledge in information repositories and technology systems. By contrast, the personalisation perspective links knowledge to the personal context and posits that it is shared through personal relationships.

While these examples do not represent the full spectrum of models, frameworks, and perspectives which abound within the KM literature, they are among the most cited models within the field.

\subsubsection{Knowledge management processes}

Alavi \& Leidner (2001) identify four fundamental processes that support organisational KM. Firstly, the creation of knowledge involves new ideas and content that requires a continual interplay between the explicit and tacit dimensions of knowledge. The second process relates to the storage and retrieval of knowledge. Depending on the knowledge type, this may occur through placing knowledge in a physical or electronic repository. Alavi \& Leidner (2001) equate the storage and retrieval of knowledge to organisational 
memory. They posit that, like individuals, organisations forget what they know and effective storage and retrieval methods can help to preserve and grow the organisational memory. The third process, knowledge transfer can occur at many levels, between individuals, from individuals to groups, across groups and across organisations ${ }^{1}$. As this process is the predominant focus of this research, knowledge sharing is discussed more fully in Section 2.3. The final KM process relates to the application of knowledge; it is from the application of knowledge that organisations derive value. Alavi \& Leidner (2001) posit that without application, the other three KM processes would not be necessary.

Nonaka (1994) identifies four different modes of knowledge conversion as shown in Figure 4. This model illustrates the way in which knowledge is created and converted as it flows through the individual, group and organisational levels.

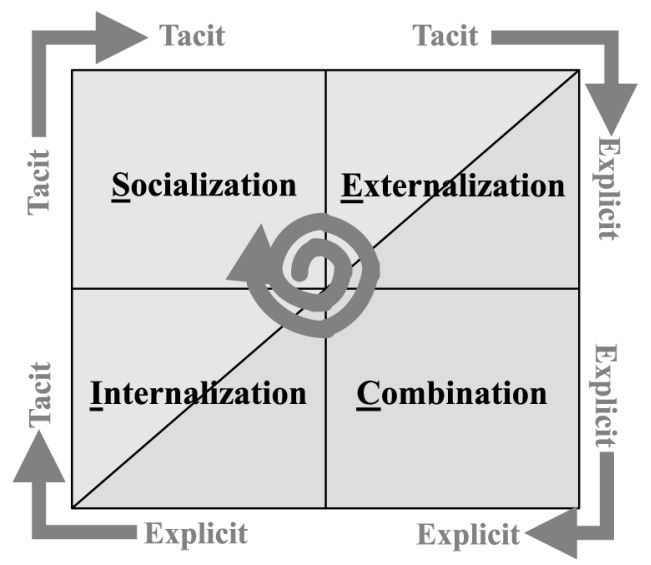

Figure 4: The SECI model (Nonaka, 1994)

In the socialisation dimension, the conversion takes place through the sharing of experiences through activities such as observation, imitation, and practice; the second dimension, externalisation requires tacit knowledge to be made explicit in some way by

The extant literature refers to both knowledge sharing and knowledge transfer, and in some cases these terms appear to have been used interchangeably. Ipe (2003) distinguishes between these two strands of the literature and notes the distinction between knowledge sharing between individuals and the concept of knowledge transfer (Chakravarthy et al., 1999) which is used predominantly to describe the movement of knowledge between larger entities within organisations. The focus of this literature review is on the knowledge sharing literature. 
sharing metaphors and analogies during social interaction. In the third dimension, combination, knowledge is exchanged and combined by way of documents, meetings etcetera; in essence, this dimension sees explicit knowledge reformatted as information. In the final dimension, internalisation, explicit knowledge is converted to tacit knowledge. Pask (1984) argues that much tacit knowledge is intuitive, and is simply "reflex" behaviour and that asking individuals to reflect and discuss their "know-how" requires an entirely different mode of thought from actual engagement in the activity. Tacit knowledge is therefore not a resource readily available to organisations, but requires some kind of process, that is, externalisation to achieve the conversion. This process requires time and money. Thus the cost of externalisation may limit the ultimate usefulness of the knowledge anyway.

Alternative views of KM processes are advanced by other scholars. Davenport and Prusak (1998) acknowledge knowledge processes to encompass the generation of knowledge, the codification of knowledge, the transfer of knowledge, and the storage of knowledge. Wiig (2002) identifies four knowledge processes: (a) building knowledge; (b) holding knowledge; (c) pooling knowledge; and (d) applying knowledge.

\subsubsection{Summary of this Section}

This section provides an introduction to the phenomenon of knowledge and its role within organisations, through the broad concept of knowledge management. A range of definitions are presented and the key knowledge management processes are discussed.

The next section introduces the reader to a comprehensive review of the literature pertaining to knowledge sharing, a subset of knowledge management, and the central focus of this study.

\subsection{Knowledge sharing}

Knowledge sharing has been discussed in the literature spanning KM, organisational behaviour, information systems, technology transfer, and innovation, as well as strategic management and organisational learning. Knowledge sharing is defined as "the voluntary interactions between human actors [through] a framework of shared institutions, including law, ethical norms, behavioural regularities, customs and so on ... 
the subject matter of the interactions between the participating actors is knowledge. Such an interaction itself may be called sharing of knowledge" (Helmstadter, 2003, p.11, cited in Wah, Menkhoff, Loh \& Evers, 2007).

Knowledge sharing deals with the ways in which knowledge may be shared between individuals, groups, or organisations (Connelly \& Kelloway, 2003) and, as more recent studies show, there is increasing interest in knowledge sharing at the interorganisational level (see Luna-Reyes \& Garcia, 2008; Mu et al., 2008; Pardo, Cresswell, Thompson \& Zhang, 2006; Willem \& Buelens, 2007).

Knowledge sharing at an individual level is defined as a voluntary act (Davenport, 1997), that can create new experience or understanding for the knowledge sharing recipient (Argote, McEvily \& Reagans, 2003; Willem, 2002). Willem (2002) states that knowledge sharing occurs between at least two parties and is a reciprocal process that allows the reshaping and sense-making of the knowledge in the new context. Knowledge sharing contains an expectation of reciprocity, and therefore differs from information sharing which can be unidirectional and unrequested (Connelly \& Kelloway, 2003). It is a dual process that enquires and contributes to knowledge stocks through activities such as learning-by-observation, listening and asking, sharing ideas, giving advice, recognising cues, and adopting patterns of behaviour (Bosua \& Scheepers, 2007).

At the team level, project teams can be particularly effective in the field of knowledge sharing through the timely integration of knowledge across organisational boundaries (Szulanski, 1996). Hoegl et al. (2003) examine the network building of individuals in innovative team projects and assert that team design and management are an important source of SC growth within organisations. By understanding team-level antecedents of network building, organisations may be able to impact those antecedents through managerial policies and the like. Similarly, Fedor et al. (2003) investigated the impact of factors related to $\mathrm{KM}$ on the responses of project team members involved with product process development. They found that knowledge dissemination was often dependent on the informal interaction between project team members, and both team leadership and organisational support had key impacts on the projects. Where leadership was low, use of tacit knowledge was high to moderate the level of leadership 
involvement. Organisational support was most effective in the dissemination of explicit knowledge.

In an organisational context, Bartol \& Srivastava (2002) define knowledge sharing as "individuals sharing organisationally relevant information, ideas, suggestions and expertise with one another". Cummings (2003) states that knowledge sharing is the means by which organisations obtain access to their own and other organisations' knowledge. The systematic sharing of knowledge is assuming a larger role in all kinds of organisations around the world (Luen \& Al-Hawamdeh, 2001; WorldBank, 2005). Bartol \& Srivastava (2002) identify four main mechanisms for individuals to share knowledge in organisations: (a) through contributions to organisational databases, (b) through formal interactions within or across teams or work unit, (c) through informal interactions among individuals, and (d) within voluntary forums such as communities of practice. The selection of knowledge sharing mechanism should depend on the type of knowledge to be shared, the routine and frequency of the sharing process, and the nature of the knowledge recipient whether at the individual, group, or organisation level (Dixon, 2000).

At the inter-organisational level, much of the theory derives from the technology transfer literature and the strategic management literature (Tang, 2008). Tang contends that studies from these fields perceive knowledge sharing to occur through contractual interorganisational relations and overlook the fact that knowledge is shared through informal interaction as well as through more formal channels. There are also examples of studies at the inter-organisational level within the knowledge transfer literature (see EasterbySmith, Lyles \& Tsang, 2008; Harryson, Dudkowski \& Stern, 2008). However, the studies detailed in the knowledge transfer literature predominantly pertain to the movement of domain knowledge between two organisations and focus on formal rather than informal mechanisms and structures (Ipe, 2003). Studies that relate specifically to the knowledge sharing literature include those of Willem \& Buelens (2007), who examine knowledge sharing in public sector organisations; Lertpittayapoom, Paul, \& Mykytn, (2007) who present a theoretical perspective on inter-organisational knowledge sharing; and Luna-Reyes \& Garcia (2008) who explore e-government and interorganisational collaboration in Mexico. The emphasis of these latter studies is less about the formal and technological mechanisms that facilitate knowledge transfer than 
the informal, social, and relational aspects of knowledge sharing that are more comprehensively detailed later in this chapter.

\subsubsection{Knowledge sharing frameworks}

Numerous factors are considered influential in the process of sharing knowledge, for example management support (Connelly \& Kelloway, 2003), trust (Cheng et al., 2008; Connelly, 2000; Mu et al., 2008; Wu et al., 2009), reward structures (Bartol \& Srivastava, 2002; Connelly, 2000), organisational culture (Bock, Zmud \& Lee., 2005; McDermott \& O’Dell, 2001; Yang, 2006), organisational structure (Greveson \& Damampour, 2007; O’Dell \& Grayson, 1998; Willem \& Buelens, 2009), ICT (Cresswell et al., 2002; Hendriks, 1999; Huysman \& Wulf, 2003), SC (Wah et al., 2007; Widen-Wulff \& Ginman, 2004) and social networks (Cross, Prusak \& Borgatti, 2001; Inkpen \& Tsang, 2005; Rush, 2001).

A number of frameworks have been developed that conceptualise knowledge sharing. Smith \& McKeen (2003) categorise factors into four distinct dimensions - social, managerial, technological and organisational. They contend that these four dimensions build on and interact with each other and create optimal conditions for knowledge sharing (see Figure 5).

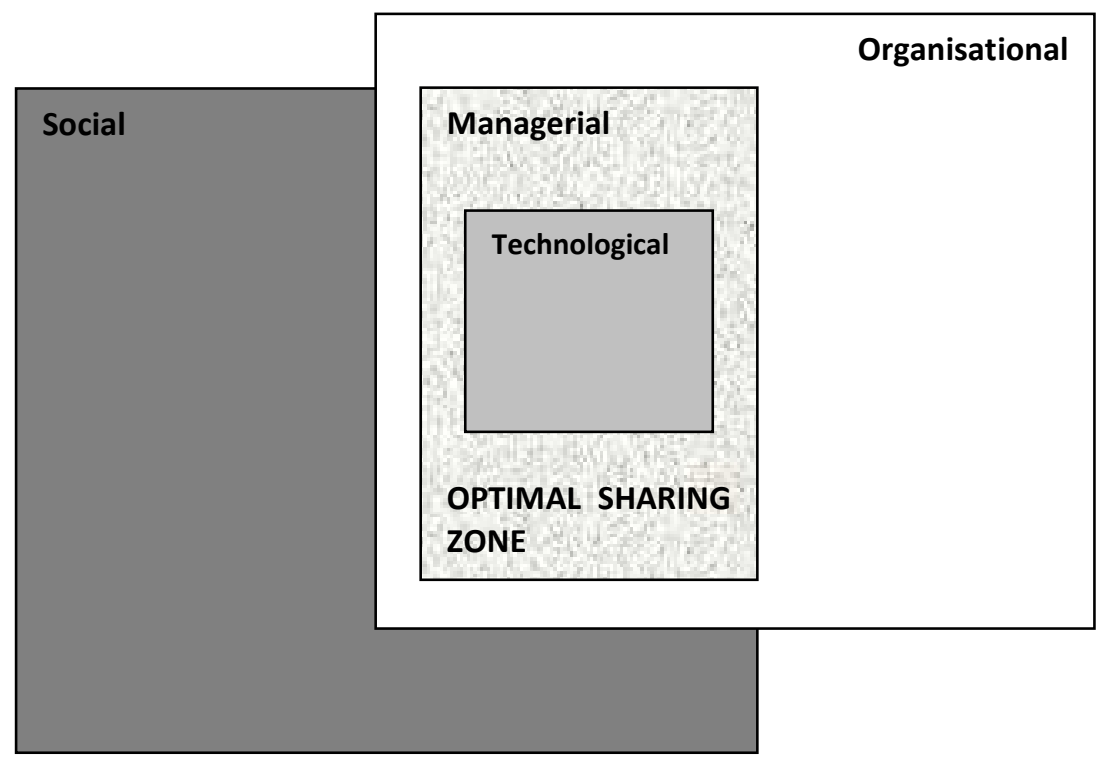

Figure 5: Optimal sharing behaviour model (Smith \& McKeen, 2003) 
The authors argue that, in the social dimension, knowledge is shared socially and includes factors such as trust, social interaction, and motivation. These factors are often considered within the greater concept of SC and have been the subject of several knowledge sharing studies (see Ipe, 2003; Mu et al., 2008; Pardo et al., 2006; Wu et al., 2009). Smith \& McKeen (2003) conclude that consideration of the social context of knowledge sharing must be a key consideration of any knowledge sharing framework. The second dimension relates to the organisational practices and processes that influence knowledge sharing behaviours. This dimension includes factors such as governance and accountability structure, enabling sharing through recognition and incentives; the way in which processes integrate knowledge; and where knowledge resources are spent. The third dimension pertains to the role of managers in leading, promoting, and influencing knowledge sharing behaviours. This dimension emphasises the important role that managers play in enabling and stifling knowledge sharing between staff. The final dimension relates to the technological context of knowledge sharing. Smith \& McKeen (2003) describe this as probably the least important motivator of knowledge sharing, but state that technology often receives considerable financial resources and must be considered an element of the knowledge sharing framework. Depending on the application, these factors may provide positive conditions for facilitating knowledge sharing, or result in limiting or prohibiting the sharing of knowledge (Smith \& McKeen, 2003).

Ipe's (2003) theoretical framework identifies six factors that influence knowledge sharing and distils these factors into four dimensions (see Figure 6). Three of the model's dimensions - the nature of knowledge, motivation to share, and opportunities to share - are encapsulated within the fourth dimension, culture of work environment. In the first dimension, the nature of knowledge shared is influenced by the tacit or explicit nature of the knowledge, as well as the value of the knowledge. In the second dimension, the motivation to share knowledge can be influenced by both internal and external factors. Opportunities to share are defined as purposive learning channels consisting of formal mechanisms such as structured work teams, technology based systems and training programmes that are designed specifically to facilitate the acquisition and dissemination of knowledge. The majority of knowledge shared through formal channels will be explicit in nature. Conversely, relational channels include personal relationships and social networks. These channels are more conducive 
to building trust and facilitating the development of respect and friendship, all of which are considered to contribute to knowledge sharing. Finally, Ipe (2003) posits that each of the factors identified in the framework will be influenced by the culture of the work environment.

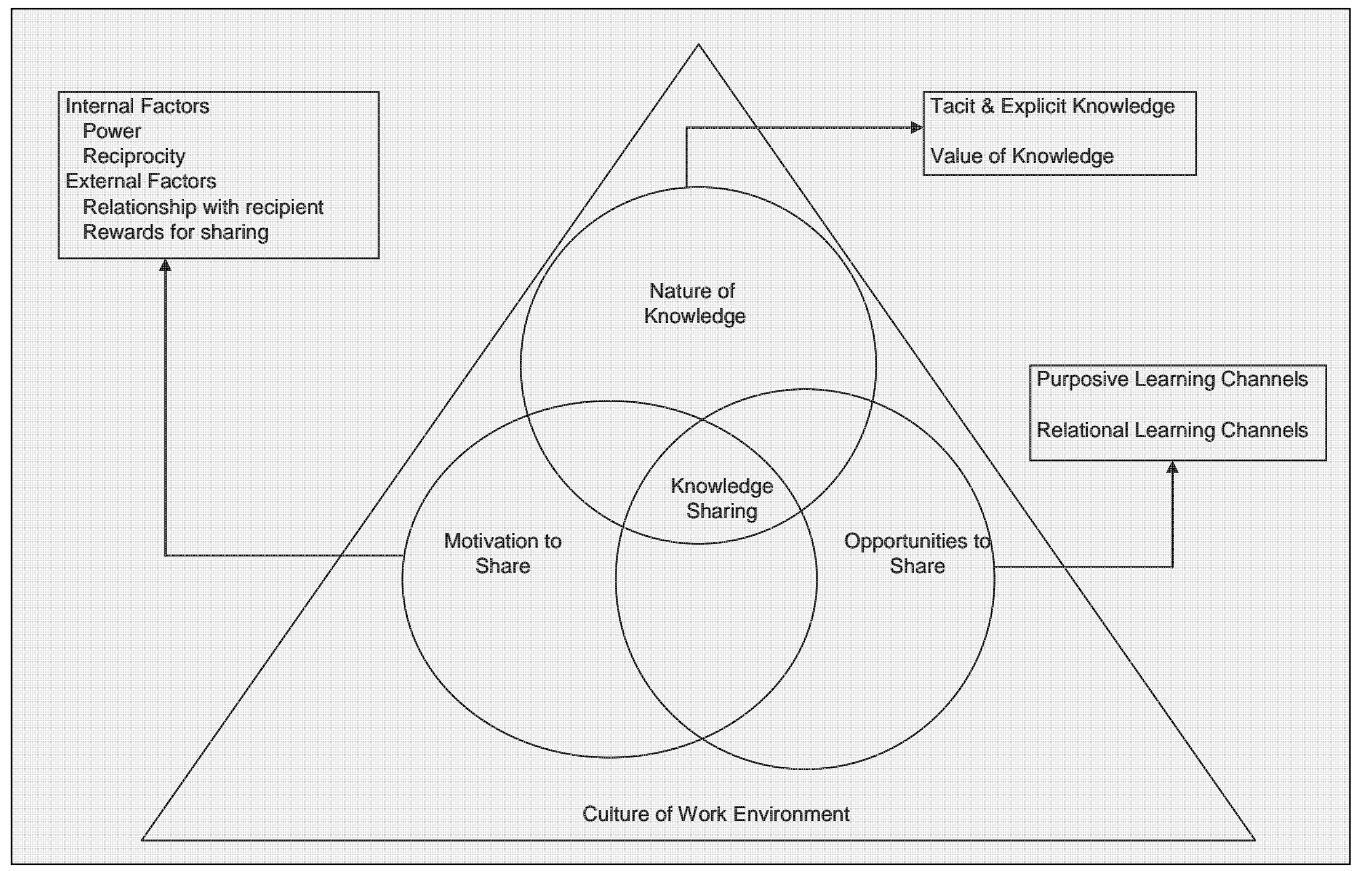

Figure 6 : Factors that influence knowledge sharing between individuals in organisations (Ipe, 2003)

In the theoretical framework proposed by Lee \& Al-Hawamdeh (2002), knowledge sharing is portrayed as a dyadic relationship between two actors. Knowledge is shared through a specific channel, but is still influenced by the confines of both the organisational and the external environments. The framework identifies a number of factors that are condensed into six components (see Table 5). The authors posit that this framework takes account of the social and economic factors that influence knowledge sharing and provides a basis on which organisations can assess the readiness of the organisation to share knowledge.

Cummings (2003) presents an alternative framework that identifies the importance of the sender and receiver and the environment in which knowledge sharing takes place. It also identifies other factors that may affect knowledge sharing, including the form and location of the knowledge, the recipient's learning disposition, and the source's knowledge sharing capability. 
Collectively, there are clear areas of convergence between the frameworks. For example, ICT and aspects related to SC such as trust and culture are recognised within each of the frameworks. However, there are differences in the extent to which individual factors are considered to play a role in knowledge sharing. Although Smith \& McKeen (2003) identify ICT as the least important dimension within their model, it is recognised as a dimension in its own right. By contrast Ipe (2003) recognises ICT as a single factor encapsulated within the greater dimension of "Opportunities to Share".

Table 5: Factors affecting knowledge sharing in an organisation (Lee \& Al-Hawamdeh, 2002)

\begin{tabular}{|c|c|c|}
\hline Component & $\begin{array}{l}\text { Factors affecting } \\
\text { knowledge sharing }\end{array}$ & Explanation of the factor \\
\hline \multirow{9}{*}{ Actor } & Communication skills & $\begin{array}{l}\text { Issues of language, grammar, adequate vocabulary, writing } \\
\text { skills and presentation. }\end{array}$ \\
\hline & People skills & $\begin{array}{l}\text { Important skills include conflict management, giving and } \\
\text { receiving feedback, consensus building, and lobbying. }\end{array}$ \\
\hline & Motivation/Initiative & Willingness to share knowledge. \\
\hline & Absorptive capacity & $\begin{array}{l}\text { The ability to identify, assicoilate, and exploit external } \\
\text { information. }\end{array}$ \\
\hline & Reputation & $\begin{array}{l}\text { The perceived reliability, trustworthiness, knowledge, and } \\
\text { prestige of the actor possessing the information. }\end{array}$ \\
\hline & $\begin{array}{l}\text { Appreciation of the } \\
\text { importance of knowledge }\end{array}$ & The perceived value of the knowledge by the other actors. \\
\hline & Incompatible personality & The way people come across. \\
\hline & $\begin{array}{l}\text { Disciplinary } \\
\text { ethnocentrism }\end{array}$ & $\begin{array}{l}\text { Actor views his/her own discipline superior and more } \\
\text { relevant than others. }\end{array}$ \\
\hline & Technophobia & $\begin{array}{l}\text { Computers and other telecommunication technologies are } \\
\text { found intimidating. }\end{array}$ \\
\hline \multirow{3}{*}{ Channel } & Document & $\begin{array}{l}\text { Any expression of human thought - pictures, graphics and } \\
\text { audio-visual materials, printed paper, computer files, etc. } \\
\text { Knowledge is fixed in a document; it cannot be modified. }\end{array}$ \\
\hline & Face-to-face unmediated & $\begin{array}{l}\text { Meetings, briefings, presentations, lectures and in-house } \\
\text { training sessions, or conversations that take place at the } \\
\text { same time and location. The recipient can request } \\
\text { clarification and customisation of the knowledge shared. }\end{array}$ \\
\hline & Face-to-face mediated & $\begin{array}{l}\text { Technology facilitates knowledge sharing and enables the } \\
\text { customisation of such knowledge in a real-time or near real- } \\
\text { time manner. Examples: videoconferencing, groupware, } \\
\text { newsgroup, virtual team rooms, email, voicemail etc. }\end{array}$ \\
\hline Knowledge & Type of knowledge & $\begin{array}{l}\text { Some knowledge is difficult to share, such as tacit } \\
\text { knowledge or knowledge lacking proven track record. }\end{array}$ \\
\hline \multirow{3}{*}{$\begin{array}{l}\text { External } \\
\text { Climate }\end{array}$} & $\begin{array}{l}\text { Economic condition of } \\
\text { the nation }\end{array}$ & $\begin{array}{l}\text { People are less inclined to share knowledge when economic } \\
\text { conditions indicate risk. }\end{array}$ \\
\hline & Government policies & $\begin{array}{l}\text { Ill justified policies may cause resentment and discourage } \\
\text { knowledge sharing. }\end{array}$ \\
\hline & Societal culture & Types of societal culture can influence knowledge sharing ${ }_{c}$ \\
\hline \multirow{8}{*}{$\begin{array}{l}\text { Organisational } \\
\text { Environment }\end{array}$} & Organisational structure & $\begin{array}{l}\text { How organisations are arranged, such as tall or flat } \\
\text { structures. }\end{array}$ \\
\hline & $\begin{array}{l}\text { Reward system and } \\
\text { incentives }\end{array}$ & $\begin{array}{l}\text { The "carrots" and "sticks" employed by the organisation to } \\
\text { stimulate knowledge sharing. }\end{array}$ \\
\hline & $\begin{array}{l}\text { Presence of knowledge } \\
\text { sharing champions }\end{array}$ & $\begin{array}{l}\text { There are individuals in the organisation who encourage } \\
\text { knowledge sharing among employees and colleagues. }\end{array}$ \\
\hline & Office layout & $\begin{array}{l}\text { The way office space is being laid out may stimulate casual, } \\
\text { non-threatening, social interaction among employees. }\end{array}$ \\
\hline & Work design & $\begin{array}{l}\text { How work is being carried out. Does it allow time for } \\
\text { reflection and sharing of ideas? }\end{array}$ \\
\hline & Staff tenure & The level of staff tenure. \\
\hline & Management support & $\begin{array}{l}\text { How visibly management supports the concept of } \\
\text { knowledge sharing through provisions in the budget for such } \\
\text { activities, raising awareness etc. }\end{array}$ \\
\hline & Organisational culture & $\begin{array}{l}\text { The way decisions are made, how competitive is the working } \\
\text { environment, the level of trust. }\end{array}$ \\
\hline
\end{tabular}


Motivation is a common factor within each of the frameworks. Knowledge sharing requires a high level of co-operative behaviour between employees (Goh, 2002), and the motivation of both the sender and the receiver is likely to greatly affect the success of knowledge sharing (Berry, 2000; Koudsi, 2000; Rappleeye, 2000 cited in Huber, 2001). In a survey of organisations undertaking $\mathrm{KM}$ initiatives, the need to motivate employees to share knowledge was identified as one of the most important challenges (Edwards, Handzic, Carlsson, \& Nissen, 2003). Motivation has been categorised as intrinsic and extrinsic. Intrinsic motivation provides an individual with a sense of immediate satisfaction and "is valued for its own sake and appears to be self sustaining" (Deci, 1976, p. 105, cited in Lam \& Lambermount-Ford, 2008). Individuals who are intrinsically motivated are more likely to generate and transfer tacit knowledge than those who are extrinsically motivated (Osterloh and Frey, 2000, cited in Lam \& Lambermount-Ford, 2008). Conversely, extrinsic motivation relates to intentional acts that are engaged in as a means to an end rather than being carried out for their own sakes (Kwok \& Gao, 2005). Extrinsic motivation is more conducive to the sharing of explicit knowledge (Lam \& Lambermount-Ford, 2008). A further point of difference is the relative importance that culture is given in each framework. While each identifies culture as an important aspect of knowledge sharing, none of the current frameworks adequately provide for the complexities of sharing between multiple organisations, where sharing is made increasingly complex due to different organisational cultures, structures, and goals (Nonaka \& Takeuchi, 1995).

\subsubsection{Knowledge sharing activities}

As discussed in the previous section Ipe (2003) identified opportunities to share as a key dimension within the framework. Jacobs \& Roodt (2007) identifies a range of knowledge sharing opportunities which are summarises as knowledge sharing activities. Their findings are summarised in Table 6 which is adapted to include opportunities identified by Ipe. One of the key factors identified by Ipe (2003), but missing from Jacobs \& Roodt's assessment, relates to knowledge sharing through technological tools. 
Table 6: Knowledge sharing activities (adapted from Jacobs \& Roodt, 2007)

\begin{tabular}{|l|l|}
\hline Knowledge Sharing Activity & Author \\
\hline $\begin{array}{l}\text { Workshops, seminars, conferences, team building } \\
\text { exercises }\end{array}$ & Gupta et al., 2000 \\
\hline Written reports & Gupta et al., 2000 \\
\hline Face-to-face interactions & Chua, 2003; loe, 2003 \\
\hline $\begin{array}{l}\text { Informal gatherings, social events, dialogues, collective } \\
\text { reflections }\end{array}$ & Ioe 2003; Yang \& Wang, 2004 \\
\hline Training & $\begin{array}{l}\text { Husted \& Michalova, 2003; loe, 2003; Yang \& } \\
\text { Wan, 2004 }\end{array}$ \\
\hline Employee suggestions/ideas programmes & Bartol \& Srivastava, 2002 \\
\hline Scheduled meetings within and across teams & Bartol \& Srivastava, 2002 \\
\hline Best practice & $\begin{array}{l}\text { Bartol \& Srivastava, 2002; McDermott \& } \\
\text { O'Dell, 2001 }\end{array}$ \\
\hline Performance appraisal, promotions, merit pay & $\begin{array}{l}\text { Bartol \& Srivastava, 2002; McDermott \&. } \\
\text { O'Dell, 2001 }\end{array}$ \\
\hline Mentoring Programmes & Gupta et al., 2000; Yang \&. Wang, 2004 \\
\hline
\end{tabular}

\subsubsection{Summary of this section}

This section introduced knowledge sharing as a subset of knowledge management. It identified the individual, team, organisational and inter-organisational levels at which knowledge sharing can take place. The section noted that, at the inter-organisational level, the majority of literature pertains to the technology transfer, strategic management and knowledge transfer literature and deals predominantly with formal and technological aspects of knowledge transfer as it occurs between organisations. Fewer studies exist within the literature specific to knowledge sharing.

The section also introduced a range of knowledge sharing frameworks. Several of these frameworks are theoretical and have yet to be considered through the gathering of empirical data. The frameworks presented address many factors and dimensions of knowledge sharing, however none of the current frameworks adequately provide for the complexities of sharing between multiple organisations, where sharing is made increasingly complex due to different organizational cultures, structures, and goals (Nonaka \& Takeuchi, 1995).

Each of the framework dimensions identified a number of factors perceived as important to the process of knowledge sharing. In the context of this research, Chapter One established that the key areas of focus for this study are those of technology and social capital. The next section discusses the framework factors that fit within the technological and social capital perspectives of this study. 


\subsection{A social capital approach to knowledge sharing}

\subsubsection{An overview of social capital}

SC was first defined by Putnam (1995, p. 664-5) who describes it as "features of social life - networks, norms, and trust - that enable participants to act together more effectively to pursue shared objectives...Social capital, in short refers to social connections and the attendant norms and trust." Other definitions of SC are shown in Table 7.

Table 7: Definitions of social capital

\begin{tabular}{|l|l|}
\hline Definition & Author \\
\hline $\begin{array}{l}\text { Social capital refers to the networks, norms, trust, and } \\
\text { mutual understanding that bind together members of } \\
\text { human networks and communities, and enable } \\
\text { participants to act together more effectively to pursue } \\
\text { shared objectives }\end{array}$ & Huysman \& Wulf, 2003 \\
\hline $\begin{array}{l}\text { Social capital as the sum of the actual and potential } \\
\text { resources embedded within, available through and derived } \\
\text { from the network of relationships possessed by an } \\
\text { individual or social unit }\end{array}$ & Nahapiet \& Ghoshal, 1998 \\
\hline $\begin{array}{l}\text { Networks together with social norms, values and } \\
\text { understandings that facilitate co-operation within or } \\
\text { among groups }\end{array}$ & OECD, 2001 \\
\hline $\begin{array}{l}\text { Investment in social relations with expected returns in the } \\
\text { marketplace whether that marketplace be economic, } \\
\text { political, labour or community }\end{array}$ & Lin, 2001 \\
\hline $\begin{array}{l}\text { The aggregate of the actual or potential resources which } \\
\text { are linked to possession of a durable network or more or } \\
\text { less institutionalised relationships of mutual acquaintance }\end{array}$ & Bourdieu, 1985 \\
\hline
\end{tabular}

SC provides access to a vast and diverse range of resources such as information (Burt, 1992), political resources (Ancona \& Caldwell, 1992), and mutual trust (Coleman, 1990).

Most often associated with sociology, the concept of SC has been used extensively in the field of corporate research (WorldBank, 2004, cited in Widen-Wulff \& Ginman, 2004). While traditionally SC has been thought of in mostly civic terms, it is now playing a growing role in understanding the nature of relations and networks between individuals and within organisations, and has been adopted as a useful theoretical approach to understanding these interactions. Consequently, SC has become a key focus of knowledge sharing research. Studies linking SC and knowledge sharing typically focus on factors including trust, behavioural norms, ties between individuals, and shared purpose and goals. Some researchers do not formally relate these factors to the concept of SC (see Bock et al., 2005; Quigley, Tesluk, Locke \& Bartol, 2007; Renzl, 
2008); others make specific connections between individual factors and the overarching concept of SC (see Mu et al., 2008; Wah et al., 2007).

In this study, SC is explored as a factor that plays a role in facilitating knowledge sharing between members of an inter-organisational team and that enables them to work together in pursuit of a common goal. Accordingly, this study adopts the SC definition posited by Huysman \& Wulf (2003).

\subsubsection{Conceptualisations of social capital and knowledge sharing}

SC has been conceptualised by several scholars, the most commonly cited of which are shown in Table 8.

Table 8: Dimensions of social capital

\begin{tabular}{|l|l|}
\hline Author & Dimension \\
\hline Coleman (1990) & $\begin{array}{l}\text { Obligations, Expectations and } \\
\text { Trustworthiness; Information Channels; } \\
\text { Norms and Effective Sanctions. }\end{array}$ \\
\hline $\begin{array}{l}\text { Aldridge et al. (2002) } \\
\text { Newell et al. (2004) } \\
\text { Woolcock (2001) }\end{array}$ & Bonding, Bridging and Linking \\
\hline Nahapiet \& Ghoshal (1998) & Relational, Cognitive and Structural \\
\hline
\end{tabular}

Coleman (1990) adopts a three dimensional approach to SC. In the first dimension, Obligations, Expectations, and Trustworthiness, SC is determined by the trust within the social structure and how much is theoretically "owed". The second dimension, Information Channels, posits that SC relates to the way in which information that flows through the social structure provide a basis for action. The third dimension relates to the Norms and Effective Sanctions that are witnessed through either a collective or individualistic structure.

Bonding, bridging and linking have also been identified as key dimensions of SC. (Aldridge, Halpern, \& Fitzpatrick, 2002; Newell, Tansley \& Huang, 2004; Woolcock, 2001). Bonding $\boldsymbol{S C}$ is characterised by strong ties among members of a group. It is most commonly found within homogenous groups, where internal cohesiveness enables the group to pursue shared goals (Newell et al., 2004). Bridging $\boldsymbol{S C}$ is depicted as ties that are weaker, less dense, but that reach a more diverse range of ties. An example of bridging SC can be found in network relationships in Silicon Valley (Lesser \& Prusak, 2003). Here, SC is not reliant on strong social ties or homogeneity; rather relationships may be weaker but still allow for growth of SC. Actors who are able to provide a 
bridging role across networks play an important brokerage role. Finally, linking $S \boldsymbol{C}$ is represented by connections between those with differing levels of power or social status. This dimension of SC is relatively new to SC taxonomies and was first introduced by Woolcock (2001). Examples of linking SC include links between the political elite and the general public or between individuals from different social classes (Aldridge et al., 2002). Bonding and bridging SC may have key roles to play in both intra-organisational collaborative projects between units, and inter-organisational projects. Whereas team members may need to use their bridging SC to access distributed knowledge, bonding SC denoted by strong bonds within teams will assist in shared understandings (Newell et al., 2004).

Nahapiet \& Ghoshal (1998) conceptualise SC through the dimensions of relational, cognitive and structural SC. These three dimensions are frequently referred to in the literature, and have two common characteristics: (a) they represent some aspect of the social structure, and (b) within that structure, they facilitate the actions of individuals (Coleman, 1990). The relational dimension of SC is associated with characteristics including trust, mutual respect and reciprocity (Huysman \& Wulf, 2003). These personal characteristics are considered to motivate individuals to share knowledge, therefore relational SC is often used to examine why people share knowledge. Wasko \& Faraj (2005) discuss how relational capital within a group will also impact on the nature of knowledge sharing. In their examination of the literature Wasko \& Faraj (2005) note two indicators of the existence of relational capital within a group. Firstly, members demonstrate a strong identification with the collective (Lewicki \& Bunker, 1996, cited in Kramer \& Tyler, 1996); and secondly, an obligation to participate in the collective is perceived (Putnam, 1995). The main function of the relational dimension of SC is to allow individuals within the structure to take action. The cognitive dimension of SC represents the resources that provide shared meaning and understanding between the network actors (Nahapiet \& Ghoshal, 1998). Analysis of cognitive SC focuses on characteristics such as shared language, shared purpose and goals. The structural dimension of SC focuses on the relationships (ties) between actors within a network. It is these ties that are deemed to create opportunities for SC transactions (Adler \& Kwon, 2001). Analysis of structural SC focuses on one or more of a range of network characteristics. Popular characteristics include network configurations and network ties. Network configurations describe the pattern of 
linkages between actors within the network and are commonly measured using network density and distance measures (Inkpen \& Tsang, 2005). These measures are fully detailed in Chapter 3.

Knowledge sharing studies commonly adopt the relational, cognitive and structural dimensions (Nahapiet \& Ghoshal, 1998) as a basis for research. Table 9 adapted from Chow \& Chan (2008) summarises research that focused on these relational, cognitive and structural dimensions to study social and knowledge sharing.

Table 9: Knowledge sharing studies focussing on social capital (adapted from Chow \& Chan, 2008)

\begin{tabular}{|c|c|c|c|c|}
\hline Source & Relational & Cognitive & Structural & Research Field \\
\hline Chua [2002] & $\begin{array}{l}\text { Trust; empathy; } \\
\text { willingness to help; } \\
\text { openness to } \\
\text { sharing/criticism; } \\
\text { group identity }\end{array}$ & $\begin{array}{l}\text { Shared language; } \\
\text { shared narrative }\end{array}$ & $\begin{array}{l}\text { Social tie } \\
\text { establishment; } \\
\text { frequency of } \\
\text { interaction }\end{array}$ & $\begin{array}{l}\text { Knowledge } \\
\text { creation }\end{array}$ \\
\hline $\begin{array}{l}\text { Hoffman \& } \\
\text { Michailova [2004] }\end{array}$ & $\begin{array}{l}\text { Social norms; } \\
\text { obligations and } \\
\text { expectations; } \\
\text { identity }\end{array}$ & & $\begin{array}{l}\text { Information } \\
\text { channel; moral } \\
\text { infrastructure }\end{array}$ & $\begin{array}{l}\text { Knowledge } \\
\text { management and } \\
\text { sharing }\end{array}$ \\
\hline $\begin{array}{l}\text { Huysman and De } \\
\text { Wit (2004) }\end{array}$ & $\begin{array}{l}\text { Mutual trust; } \\
\text { norms; obligations } \\
\text { and identification, } \\
\text { motivation }\end{array}$ & $\begin{array}{l}\text { Shared codes and } \\
\text { language; shared } \\
\text { narratives }\end{array}$ & $\begin{array}{l}\text { Network ties; } \\
\text { network } \\
\text { configurations; } \\
\text { appropriable } \\
\text { organisation }\end{array}$ & Knowledge sharing \\
\hline $\begin{array}{l}\text { Inkpen \& Tsang } \\
\text { [2005] }\end{array}$ & Trust & $\begin{array}{l}\text { Shared goals, } \\
\text { shared culture }\end{array}$ & $\begin{array}{l}\text { Network ties, } \\
\text { network } \\
\text { configurations, } \\
\text { network stability }\end{array}$ & $\begin{array}{l}\text { Knowledge } \\
\text { Transfer }\end{array}$ \\
\hline Lang (2004] & $\begin{array}{l}\text { Genera lised trust; } \\
\text { reciprocity }\end{array}$ & Value introjection & Bounded solidarity & $\begin{array}{l}\text { Knowledge } \\
\text { integration }\end{array}$ \\
\hline Liu \& Besser (2003) & $\begin{array}{l}\text { Generalised trust; } \\
\text { norms or } \\
\text { expectations }\end{array}$ & & Social ties & Knowledge sharing \\
\hline $\begin{array}{l}\text { Nahapiet \& } \\
\text { Ghoshal (1998) }\end{array}$ & $\begin{array}{l}\text { Trust; norms; } \\
\text { obligations and } \\
\text { expectations; } \\
\text { identification }\end{array}$ & $\begin{array}{l}\text { Shared codes and } \\
\text { language; shared } \\
\text { narratives }\end{array}$ & $\begin{array}{l}\text { Network ties, } \\
\text { network } \\
\text { configurations, } \\
\text { appropriable } \\
\text { organisation }\end{array}$ & $\begin{array}{l}\text { Knowledge } \\
\text { exchange and } \\
\text { creation }\end{array}$ \\
\hline $\begin{array}{l}\text { Wasko \& Faraj } \\
{[2005]}\end{array}$ & $\begin{array}{l}\text { Commitment; } \\
\text { reciprocity }\end{array}$ & $\begin{array}{l}\text { Self-rated } \\
\text { expertise; tenure } \\
\text { in the field }\end{array}$ & Centrality & $\begin{array}{l}\text { Knowledge } \\
\text { contribution }\end{array}$ \\
\hline $\begin{array}{l}\text { Tsai \& Ghoshal } \\
\text { (1998) }\end{array}$ & $\begin{array}{l}\text { Trust and } \\
\text { trustworthiness }\end{array}$ & Shared vision & Social interaction & $\begin{array}{l}\text { Resource exchange } \\
\text { and value creation }\end{array}$ \\
\hline $\begin{array}{l}\text { Yii-Renko et al. } \\
\text { [2001] }\end{array}$ & & & $\begin{array}{l}\text { Social interaction; } \\
\text { relationship quality; } \\
\text { customer network } \\
\text { ties }\end{array}$ & $\begin{array}{l}\text { Knowledge } \\
\text { acquisition and } \\
\text { exploitation }\end{array}$ \\
\hline $\begin{array}{l}\text { Chow \& Chan } \\
\text { [2008] }\end{array}$ & Trust & Shared goals & $\begin{array}{l}\text { Network } \\
\text { configuration }\end{array}$ & Knowledge sharing \\
\hline
\end{tabular}

\subsubsection{Relational social capital factors}

The relational dimension of SC is concerned with the why and when of knowledge sharing (Huysman \& Wulf, 2003). In the relational dimension, the most commonly studied factors in knowledge sharing research are trust; norms, obligations and/or expectations; and identity (see Figure 7). 


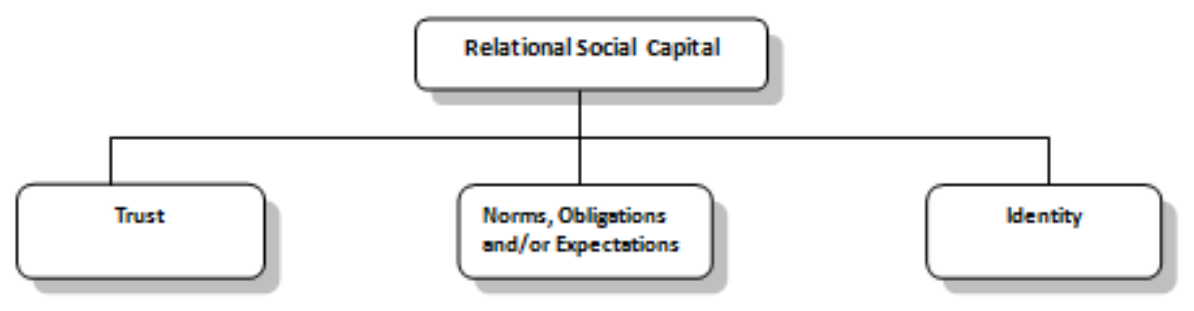

Figure 7: Relational social capital

Studies vary in terms of scope, with some studies focussing on trust, for example as a single factor (see Chow \& Chan, 2008; Inkpen \& Tsang, 2005), while others focus on specific aspects of trust such as mutual trust (see Tsai \& Ghoshal, 1998); others focus on the interplay between multiple factors within a single dimension (see Liu \& Besser, 2003) or across multiple dimensions (see Huysman \& de Wit, 2004; Wasko \& Faraj, 2005).

Trust

Trust is one of the most common factors identified in the relational dimension of SC and is viewed as critical to the development of relationships (Cowles, 1997; Lewicki, McAllister, \& Bies, 1998). There is substantive evidence that where individuals are engaged in a trusting relationship, they are more willing to engage in social exchange and cooperative action (Nahapiet \& Ghoshal, 1998). Further, where high levels of trust exist, individuals are more willing to take risks in knowledge exchange, which may represent an increased willingness to share different types of information (Nahapiet, 1996; Ring \& Van de Ven, 1992, cited in Nahapiet \& Ghoshal, 1998). Molm, Takahashi, \& Peterson (2000) argue that the existence of trust between team members is more important in facilitating knowledge sharing than any fiscal or economic reward, even when that reward is known prior to the exchange. As two parties enter a trust relationship, willingness to share resources, such as information and knowledge is increased as trust is built (Nahapiet \& Ghoshal, 1998).

Trust and its relationship to knowledge sharing has been researched at the individual level (see Abrams, Cross, Lesser, \& Levin, 2003; Wu et al., 2009), at the team level (see Wu et al., 2007; Renzl, 2006; Molm et al., 2000), the organisational level (see Inkpen \& Tsang, 2005; Al-Alawi, Al-Marzooqi, \& Mohammed., 2007; Alam, Abdullah, Ishak, 
\& Zain, 2009) and at the inter-organisational level (see Pardo et al., 2006; Willem \& Buelens, 2007; Luna-Reyes et al., 2008; Mu et al., 2008).

Lin (2001) defines trust as confidence or expectation that an individual will take other individuals' interests into account in exchanges. Trust is conducive to social activities and allows people to co-exist without the continual need for conflict or negotiation (Cohen \& Prusak, 2001). There is debate however, as to whether trust is an outcome of SC (Woolcock, 2001), that is, when SC grows then trust is developed, or, conversely, that trust is more an enabler of SC development (Healy et al., 2001). In Fukuyama's (1995) view, at a macro or national level, high trust societies are more likely to develop high SC resulting in high economic growth than low trust societies. This view lends weight to Healy, Cote, Helliwell, \& Field's perspective that trust is an enabler of SC. Similarly Cohen \& Prusak (2001) maintain that trust is a building block for the development of relationships, communities, and cooperation which, in turn, become the basis of SC.

Scholars have identified various types of trust, as shown in Table 10. Putnam (1995) identifies "thin" and "thick" trust. Thin trust refers to a more generalised trust that exists between members of a community and is less stable than thick trust which exists as a property of intimate social networks and, as such, has a higher degree of stability or certainty. Similarly, Ring and Van den Ven (1994) categorise thin trust as fragile and posit that it is more uncertain than resilient (thick) trust. Sako (1992, cited in Newell et al., 2003) identifies three trust categories. The first relates to contractual trust. In this category, trust is viewed as formal agreements that bind together two or more parties; the second category relates to competence and the expectation that an individual will perform their role to the desired level of proficiency; the third category, goodwill trust, refers to mutual expectations of open commitment, described by Green (2003) as the willingness to do more than is formally expected.

The notion of altruistic trust is less well established within the literature. $\mathrm{Wu}$ et al. (2009) posit that for individuals with a high degree of altruism, trust in colleagues is not a critical consideration when sharing knowledge. Noteboom's (2002) approach to altruistic trust suggests that trust can be based on several factors including personal interest, opportunism or the lack of an alternative, but true altruistic trust goes beyond these factors and comprises sincerity even if opportunism is apparent. Like the concept 
of goodwill trust, posited by Sako (1992), altruistic trust suggests that an individual will act without self-concern or self-interest. Shapiro's trust model (1992, cited in Ratnasingham, 1998) defines deterrence-based trust as based on the fear of punishment should trust be violated. Thus individuals are deterred from violating a trust relationship by the perceived negative consequences of their actions. Knowledge-based trust relates to a relationship that has been established over time and in which an individual party has established a degree of knowledge of the other party which enables them to predict the party's likely behaviour. Shapiro's third trust type, identificationbased trust, is denoted by common values and empathy between individuals. Zucker's (1986) typology describes process-based trust as tied to the expectation of ongoing change, expected or past exchange. Characteristic-based trust relates to trust that is developed through similarities between individuals or parties, such as ethnicity or background, while institutional-based trust denotes similarity at an institutional, or formal societal, rather than an individual level (Zucker, 1986). Finally, Jones and George (1998) postulate that while the presence of conditional trust will support a group to work towards a common goal, unconditional trust has the potential to increase the quality of the experience and can support the evolution of a group of individuals into a unified team.

Table 10: Typologies of trust (adapted from Newell et al., 2003)

\begin{tabular}{|l|l|}
\hline Typologies of Trust & Source \\
\hline $\begin{array}{l}\text { Thick Trust } \\
\text { Thin Trust }\end{array}$ & Putnam, 1995 \\
\hline $\begin{array}{l}\text { Fragile, easily broken trust } \\
\text { Resilient trust }\end{array}$ & Ring \& Van de Ven, 1994 \\
\hline $\begin{array}{l}\text { Contractual agreement binding parties } \\
\text { Competency based belief } \\
\text { Belief in goodwill }\end{array}$ & Sako, 1992 \\
\hline $\begin{array}{l}\text { Altruistic trust } \\
\text { Deterrence-based trust } \\
\text { Knowledge-based trust } \\
\text { Identification-based trust }\end{array}$ & Wu et al., 2009 \\
\hline $\begin{array}{l}\text { Process-based (reciprocal, recurring exchange) } \\
\text { Characteristic-based (social similarity) } \\
\text { Institutional-based (expectations based on social } \\
\text { similarity }\end{array}$ & Zucker, 1986 \\
\hline $\begin{array}{l}\text { Conditional trust } \\
\text { Unconditional trust }\end{array}$ & Shapiro et al., 1992 \\
\hline
\end{tabular}

High levels of trust are very important to facilitate the sharing of tacit knowledge among teams (Newell et al., 2002), and high levels of SC are identified as a mechanism to reduce the transaction costs of teamwork (Cohen \& Prusak, 2001). Within organisations, the building of trust is a mechanism through which intra- and inter-team 
relationships can be forged. Focussing on the development of trust within teams, Jones \& George (1998, in Newell et al., 2002) argue that unconditional trust is preferable in terms of building enduring synergistic team relationships, and will positively affect the creation and sharing of knowledge. However, trust does not automatically occur within relationships, it grows and develops over time, and requires a foundation for the initial trust to build from. Goodwill trust, as identified by Sako (1992) is based on one's belief that another individual will act in one's interests. It follows the principle, "treat others as you wish to be treated yourself'. Goodwill trust can be established through repeated informal networks. Davenport \& Prusak (1998) offer three suggestions to build trust in the workplace. Firstly, trust must be visible. If staff are actively engaging in knowledge sharing, then credit or acknowledgement of some kind must be given. This will strengthen reciprocity. Secondly, trust must be ubiquitous and encompass all individuals. Finally, trust flows downward; therefore trust must come from top management.

Trust can be particularly important where knowledge sharing occurs between individuals involved in inter-organisational relationships (Luna-Reyes, Creswell, \& Richardson, 2004), or where team members from individual organisations are brought together for the purposes of a specific collaborative venture. Each actor belongs to an individual organisation with its own set of values, norms, and beliefs, and the onus is on the newly formed inter-organisational team to develop its own SC, shared norms, trust, and belief in order to work together cohesively.

\section{Norms, obligations and expectations}

Studies have posited a relationship between knowledge sharing social norms, or a sense of obligation or expectation (Huysman \& de Wit, 2004; Liu \& Besser, 2003; Nahapiet \& Ghoshal, 1998). Norms, and obligations and expectations are commonly studied within the relational dimension as either a single integrated factor (Liu \& Besser, 2003) or as individual factors (Hoffman \& Michailova, 2004, cited in Chow \& Chan, 2008; Nahapiet \& Ghoshal, 1998).

Coleman (1990) defines norms as indicating a degree of consensus and as reflecting the values of the community. In this sense, norms are constructed through group agreement rather than through the power or control of any single individual. Obligations and 
expectations relate to the potential undertaking of some activity in a future context (Coleman, 1990; Nahapiet \& Ghoshal, 1998). This may manifest as commitment at the individual or organisational level (Scarbrough \& Carter, 2000). Coleman defines commitment as a duty or obligation that arises from frequent interaction and denotes an intention to engage in future action (Coleman 1990). Scarbrough \& Carter (2000) posit that the level of commitment to knowledge sharing is strongly related to successful KM outcomes. Meyer and Allan (1997, cited in van den Hooff \& Ridder, 2004) identify three commitment types: affective, continuance, and normative commitment. Affective commitment is related to an individual's identification with an organisation or feeling of emotional connection to the organisation. Continuance commitment relates to the high costs of exiting an organisation thereby discouraging an individual to leave the organisation. Normative commitment describes an individual's sense of obligation towards an organisation that results in the feeling that an individual is duty bound to stay with an organisation.

Other research has shown that individuals who engage in social interaction do so based on an expectation of social reward, such as enhanced reputation, approval, or respect (Blau, 1964, cited in Wasko \& Faraj, 2005). Rather than financial reward, it is the context of building SC that is more likely to facilitate the knowledge exchange. Similarly, an individual's reputation reflects SC which can be used to generate certain returns (Lin, 2001).

\section{Identity}

Affective commitment (described above) is also associated with an individual's sense of identity with a team, group or organisation. Identity is defined as the process whereby individuals see themselves "at one" with another person or a social group (Nahapiet \& Ghoshal, 1998), or the extent to which individuals perceive their connections to other individuals (Widen-Wulff \& Ginman, 2004).

Social Identity Theory (SIT) conceptualises identity as concerned with how individuals develop a sense of membership and belonging to different groups (Tajfel, 1982; Tajfel \& Turner, 1982). SIT comprises two distinct components: personal identity encompassing individual characteristics, and social identity relating to an individual's group classification. Ashforth and Mael (1989) contend that together, these two 
components define social identification as the perception of oneness with, or the sense of belonging to, some human collective. Drawing on SIT, Mueller, Renzl \& Kaar (2008) posit that a lack of common identity keeps employees from actively contributing to and exchanging knowledge.

Though identity is referenced briefly by Huysman and de Wit (2004), it is more commonly associated with studies of knowledge creation and exchange rather than knowledge sharing, (see Chua, 2001; Nahapiet \& Ghoshal, 1998).

\subsubsection{Cognitive social capital factors}

The cognitive dimension of SC relates to the ability to understand what is being shared, or as Huysman and Wulf (2004) describe it the "analysis of what" is shared. As shown in Figure 8, this dimension encompasses factors such as shared culture (see Inkpen \& Tsang, 2005; Kekale, Takala \& Ajmal, 2008; Kim \& Lee, 2006; Smith \& McKeen, 2003; Syed-Ikshan \& Rowland, 2004), shared purpose and goals (see Chen, Lin, Liou, \& Liu, 2009; Inkpen \& Tsang, 2005; Kim, Lee, \& Kim., 2008; Nahapiet and Ghoshal, 1998); and shared language (see Nahapiet \& Ghoshal, 1998; Tsai \& Ghoshal, 1998).

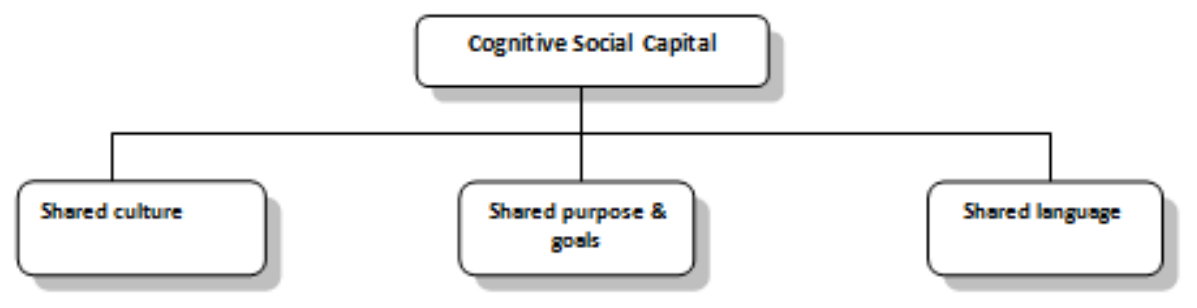

Figure 8: Cognitive social capital

Although these factors are often examined as discrete elements, other scholars encompass them into the overarching factor of culture (Kim \& Lee, 2006; Van den Hooff \& Huysman, 2009).

\section{Shared culture}

Issues of organisational culture and their relationship to knowledge sharing have been explored by several scholars (see Connelly \& Kelloway, 2003; DeLong \& Fahey, 2000; McDermott \& O’Dell, 2001; Parker \& Bradley, 2000; Smith \& McKeen, 2003; Van den Hooff \& Huysman, 2009). In the organisational setting, culture is described as the set of forms and values which collectively guide the behaviour of employees (Smith \& 
McKeen, 2003). Moreover, Smith \& McKeen point out that while organisational culture will set the predominant tone for the way in which people behave, multiple cultures at the business unit and individual levels may exist within the organisation. Sveiby and Simons (2002) identify the business unit, immediate superior, and coworkers as additional cultural influences.

Establishing a knowledge-friendly or shared culture has a beneficial effect on knowledge sharing (Van den Hooff \& Huysman, 2009). Davenport et al. (1998, cited in Van den Hooff \& Schipper, 2009) characterise such a culture as one where curiosity and a willingness to learn and explore create a positive orientation toward knowledge sharing. As detailed earlier in this chapter, Smith \& McKeen (2003) contend that a blend of organisational, managerial, social, and technological factors contribute to the development of a knowledge sharing culture.

The concept of a sharing culture between business teams in a single public sector organisation in Malaysia is explored by Syed-Ikshan \& Rowland (2004), who found a positive relationship between the existence of a knowledge sharing culture and the level of knowledge transfer.

\section{Shared purpose and goals}

Inkpen and Tsang (2005) posit that the presence of shared purpose and goals ${ }^{2}$ provides individuals with similar understandings of how to interact with each other. Further, they contend that this sense of shared purpose acts as a "bonding mechanism" that assists different parts of a network to integrate knowledge. In their 2008 study, Chow \& Chan found that shared goals directly influence the attitude and subjective norm about knowledge sharing, and also indirectly influence the intention to share.

With regard to the organisational level, Kanter, Stein, and Jock (1992, cited in Kim \& Lee, 2006) suggest that when the organisation's purpose is clear then this can assist in the achievement of organisational goals. Further, this can facilitate a sense of involvement and contribution among employees (Davenport, Jarvenpaa \& Beers, 1996; O’Dell \& Grayson, 1998; Popovich 1998, cited in Kim and Lee, 2006).

\footnotetext{
${ }^{2}$ Also referred to as shared vision (Tsai \& Ghoshal, 1998)
} 
At an inter-organisational level, conflict may arise from collaboration partners having contradictory goals or goals inconsistent with those of other partners (Anderson, 1990, cited in Inkpen \& Tsang, 2005).

\section{Shared language}

Nahapiet \& Ghoshal (1998) present an analysis of the way in which shared language can influence the conditions for knowledge exchange. Firstly, they posit that language provides the fundamental mechanism by which social interaction can take place, "It is the means by which people discuss and exchange information, ask questions, and conduct business in society" (Nahapiet \& Ghoshal, 1998, p. 253). Secondly, language provides a filter through which individuals perceive and make sense of the environment. Thirdly, shared language supports the development of new concepts and ideas by enhancing combination capability. Thus, the bringing together of knowledge, aided by shared language, assists with the creation and exchange of new knowledge.

\subsubsection{Structural social capital factors}

Huysman and Wulf (2003) describe the structural dimensional of SC as the analysis of who shares knowledge and how it is shared. In the context of social networks, studies of structural SC have focused on several factors, including network configuration (Chow \& Chan, 2008; Huysman and de Wit, 2004; Inkpen \& Tsang, 2005; Nahapiet \& Ghoshal, 1998), and network ties (Huysman and de Wit, 2004; Inkpen \& Tsang, 2005; Liu \& Besser, 2003; Nahapiet \& Ghoshal, 1998; Seibert \& Liden, 2001) as shown in Figure 9.

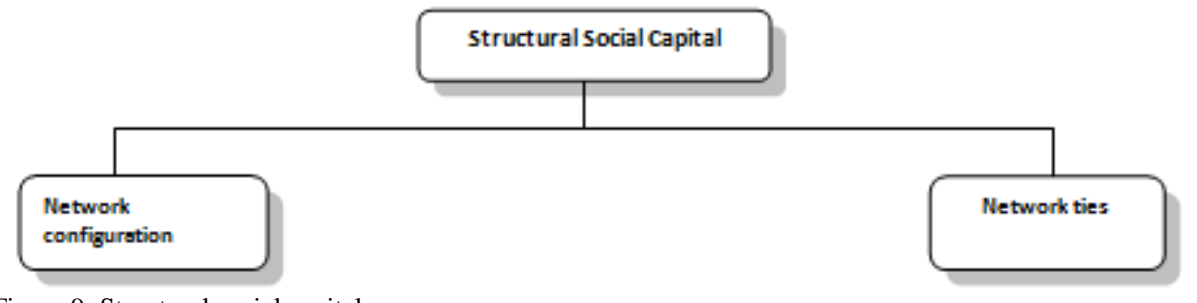

Figure 9: Structural social capital

\section{Network configuration}

Network configuration represents the pattern of linkages among network members (Inkpen \& Tsang, 2005). Configuration is constituted by a number of factors including hierarchy, density and connectivity (Krackhardt, 1992, cited in Inkpen \& Tsang, 2005; 
Nahapiet \& Ghoshal, 1998). Studies of network configuration have been used to compare the formal structure of teams and organisations to the informal networks that underlie that structure (Hansen, 2002; Mead, 2001).

\section{Network ties}

A network or relational tie refers to actors linked by social ties. Although there is an extensive range of social ties, essentially a tie establishes a link between a pair of actors. It is through these relational ties that actors may exchange resources such as information, goods and services, or financial support (Haythornthwaite, 1999). Hansen (1999) studied 120 new product development projects and showed that weak and strong ties between organisational subunits provided difference advantages and disadvantages in terms of the search and transfer of knowledge.

\subsubsection{Social networks}

A key area of interest between SC research and knowledge sharing research lies in the study of social networks. A social network is described by Haythornthwaite (1999) as a set of social entities, for example individuals, groups, or organisations that are connected to each other in order to exchange information or other resources. A social network consists of a finite set or sets of actors and the relation or relations defined between them (Wasserman \& Faust, 1994). In order to better understand the influence between SC and knowledge sharing, a body of research has begun to grow that explores SC and knowledge sharing through the lens of social networks.

In a survey of individuals involved in $\mathrm{KM}$ work, $\mathrm{SC}$ and networking were identified as being amongst the most important factors to be considered (Murray \& Myers, 1997). Some companies recognise the value that the presence of SC between workers can provide in terms of knowledge sharing, and are attempting to facilitate social networks by providing employees with opportunities to interact more frequently (Flaherty, 2000, cited in Connolly \& Kelloway, 2001). These opportunities can serve to boost morale within an organisation as well as enable workers to relax and get to know each other without the pressure of getting work done. Castells (1996) states that through knowledgeable people being better connected with other similarly knowledgeable people there has been an increase in the degree to which knowledge is distributed in dense networks. Introducing the concept of 'knowledge networks', Johnson (2009) 
posits that understanding knowledge networks is fundamental to moving beyond IT to understanding the deeper social aspects of knowledge management.

Fukuyama (2000, p. 199) defines a network not in terms of a formal organisation, but in terms of SC and states that "a network is a group of individual agents who share informal norms or values beyond those necessary for ordinary market transactions." Thus, it is the capability of competent participation within the complex webs of relationships among people and activities that means much more than the simple possession of a store of knowledge (Gerardi et al., 1998, cited in Jorgenson, 2004). Further, Fukuyama contends that organisations that maintain centralised management structures with authoritarian hierarchies experience increasing challenges because this structural form cannot cope with today's complex informational needs. This view is supported by Tsai's study (2002) of knowledge sharing in intra-organisational business units which examined how formal hierarchical structures and informal lateral relations influence knowledge sharing. The results of the study confirmed that the centralised, formal hierarchical structure had a negative effect on knowledge sharing, while informal lateral sharing had a significant positive effect on the sharing of knowledge between business units. Thus, as Aldridge et al. (2002) conclude, such hierarchical or unequal structures do not provide a good foundation for the building of SC (Aldridge et al., 2002). Fukuyama (2000) explains that to operate successfully in an authoritarian environment, a leader must have total knowledge of all that he/she presides over. However, as organisations grow increasingly complex and rely more heavily on technology, maintaining complete knowledge over all aspects of a business is not possible. Therefore, organisations need to decentralise in terms of managerial authority. This move away from bureaucracy to more decentralised organisations opened the way for the evolution of social networks.

Traditionally, organisations have been analysed through the lens of organisational structure. Robbins \& Decenzo (2001) identified three fundamental elements denoting organisational structure; centralisation, formalisation, and integration. They define centralisation as the degree to which decision making is located in the higher levels of a hierarchical relationship. Formalisation refers to the degree to standardisation, and processes and rules that are in place to guide employee behaviour and conduct of activities (Robbins \& Decenzo, 2001). Integration describes the extent to which various 
organisational business units work in an inter-related fashion. Increasingly the focus of attention is moving towards gaining an understanding of the social or informal networks that are now considered to provide clearer understandings of how work "actually" gets done within organisations (see Inkpen \& Tsang, 2005; Kim \& Lee, 2006; Mu et al., 2008; Tsai, 2002).

Zack (2000, p. 1) identifies several network types when he defines organisational structure or form as "the pattern of connections and interdependencies among organisational members". These include the formal organisation structure (who reports to whom), the informal organisation structure (who actually communicates and exchanges information with whom), the structuring of work (who depends on whom), or the social relationships (who likes whom, who is similar to whom). Although Zack posits a distinction between informal and social networks, it is argued that within an informal relationship, there will be a degree of social connection to the extent that the motivating behaviour that drives the voluntary communication and exchange is likely to be one of mutual trust and reciprocity. Increasingly, it is through these informal networks, rather than through traditional organisational hierarchies, that knowledge is shared (Cross \& Prusak, 2002). Consequently, consideration of knowledge sharing from the viewpoint of informal networks may be seen as an inherently social process whereby knowledge will be shared predominantly through social networks and relationships rather than through a specific technology channel (Davidson \& Voss, 2003).

In order to understand and aid informal networks, Cross et al. (2002) identify four areas for consideration: (a) the formal structure of the organisation, (b) work management practices, (c) employee management practices, and (d) cultural values. They recommend that informal networks can be facilitated by organisational leaders by creating time and space for cross-unit collaboration, by focusing on developing relationships within the work context rather than through off-site specific team building exercises, by hiring individuals who can demonstrate a commitment to collaboration and rewarding that behaviour, and, finally, by recognising and rewarding individuals who involve others in problem solving. 


\subsubsection{Measuring social capital}

In their examination of the SC literature, Widen-Wulff \& Ginman (2004) identified several key studies and measures as shown in Figure 10. The authors posit that measurement of SC is difficult given that definitions of SC are not only multidimensional, but also include several levels of analysis. For the most part, measurement of SC has been concentrated at the macro level, where measures focus on quantifying SC and how it contributes to economic development. At the individual or at the group level, the most common measures relate to an individual's membership in informal and formal associations and networks and the trust, norms, and values that enable exchanges and lower transaction costs. Borgatti, Jones \& Everett (1998) contend that this variation has, in fact, hidden another difference in terms of outward and inward focus. In their view, research that focuses on the individual looks to ties outside the individual, whereas at group level the focus has been to identify only the ties within the group.

\begin{tabular}{ll}
\hline Examples of Social Capital Measures & \\
\hline Putnam [53] & $\begin{array}{l}\text { organization of society } \\
\text { citizens' involvement in society actions } \\
\text { voluntary actions } \\
\text { informal socializing } \\
\text { social trust } \\
\text { horizontal associations } \\
\text { social integration } \\
\text { membership in informal and formal associations and networks } \\
\text { norms, values that facilitate exchanges, lower transaction costs } \\
\text { Woolcock \& Narayan [50] } \\
\text { attitudes } \\
\text { values } \\
\text { membership, participation } \\
\text { trust } \\
\text { structural vs cognitive social capital (norms, values, attitudes, beliefs) } \\
\text { horizontal vs vertical organizations (horizontal networks contribute to SC, vertical } \\
\text { relationships inhibit it) } \\
\text { heterogeneous vs homogeneous organizations } \\
\text { formal vs informal organizations } \\
\text { emotional intensity care }\end{array}$ \\
Krishna \& Schrader [51] & \\
\hline
\end{tabular}

Figure 10: Examples of social capital measures (Widen-Wulff \& Ginman, 2004, p. 452)

Krishna \& Scrader (2002) developed a qualitative measurement tool based on the relational, cognitive, and structural dimensions of SC. In the structural dimension, they posit that the tool will show the infrastructure of the group and generate knowledge about the structural mechanism of knowledge sharing. For the content dimension, Krishna \& Scrader posit that four aspects of knowledge sharing must be considered: information exchange, problem identification, behaviour regulation and conflict management. For the relational dimension, Tyler \& Blader (2001, cited in WidenWulff \& Ginman, 2004) have designed a measurement tool to explore people's behaviour engagement within groups. 
Many researchers employ SNA to examine social networks (see Cross et al., 2002; Hansen, 1999; Haythornthwaite, 1996; Mead, 2001). SNA is the study of social relations among a set of individuals (actors) and seeks to understand beliefs and behaviours as a function of the structure of relationships in which they occur. SNA has been identified as a useful tool to explore SC aspects of knowledge sharing. SNA can be used to identify knowledge flows along existing pathways in organisations; to understand the knowledge flow and find out what the patterns are; and to apply interventions to create, reinforce, or change the patterns (Anklam, 2003). Within a team environment, SNA has been identified as particularly useful in analysing the unique set of network attributes that are in place, such as individuals' roles, their network linkages, and key network metrics such as the size and density of the networks (Mead, 2001). These attributes are of specific importance to a project team that characteristically combines a number of individuals from different units or organisations who are tasked with the completion of a specific project within a strict timeline. As individuals leave and join the project, SNA can be useful in quickly identifying the pattern of communication and potential knowledge sharing within the project team (Anklam, 2003).

Borgatti \& Cross (2003) use SNA to form a model of information seeking that is based on a relational view. They propose that the likelihood of actors seeking information from other actors is based on four factors: (a) knowing what the person knows, (b) the value they place on that person's knowledge, (c) whether they are able to gain access to that person's thinking within the necessary timeframe; and (d) their perception of the cost of seeking information from that person. They collected data from separate groups within two organisations, and conducted preliminary interviews to form an understanding of the background and purpose of the groups. Following this, data were collected through electronic surveys. The two sets of data were then analysed to form the relational model which was then tested using network correlation and regression. The findings of the study supported the model and the authors suggested that their work provides a platform for further work and suggest SC as an area of potential interest.

Haythornthwaite's 1999 study of the relationship between tie strength and media choice found that where strong ties existed, the organisationally-established communication channel would be the first choice. However, strongly tied pairs will also explore other 
communication channels in order to facilitate their need to communicate. By comparison, weakly tied pairs are more likely to depend on the organisationallyestablished communication channel and are less likely to deviate from that.

\subsubsection{Summary of this section}

This section introduced the concept of social capital and provided a range of definitions through which social capital has been characterised. The section identified and detailed several conceptualisations of social capital and identified the relational, cognitive and structural dimensions espoused by Nahapiet \& Ghoshal (1998) as a popular framework that has been widely used within the literature linking social capital and knowledge sharing. Specific factors relating to each of these dimensions are identified and discussed and the concept of social networks is introduced.

The section also identified social capital as an important aspect of knowledge sharing, and one that is gaining increasing importance in research into the phenomenon particularly in relation to corporate research.

The section also outlines a variety of approaches to measuring social capital. Though measurement has proved problematic, the use of social network analysis has provided scholars with a useful tool for providing both quantitative and qualitative data pertaining to knowledge sharing.

\subsection{A technological approach to knowledge sharing}

ICT and its use as a knowledge sharing tool has been explored in a number of studies (Hendriks, 1999; Huber, 2001; Kakabadse et al., 2003; Smith \& McKeen, 2003), and has been suggested as useful in supporting knowledge sharing skills and empowering individuals (Hendriks, 1999). More specifically, Hendriks (1999) postulates that ICT can be effective in lowering some of the barriers to knowledge sharing; by facilitating access to explicit information bases; by improving knowledge sharing processes; and, lastly, by helping to locate meta-knowledge relevant to the process of knowledge sharing. However, this stance is challenged by many authors (see Cross \& Baird, 2000; Ruggles, 1998; Smith \& McKeen, 2003) who identify ICT as an enabler of knowledge sharing, rather than a driver. 
More common uses of ICT-related initiatives focus on the coding and sharing of best practice, and the creation of both organisational knowledge directories and knowledge networks. ICT is also used in an attempt to capture and process the knowledge of individuals, so that it may be applied in new contexts (Newell et al., 2002). The most common ICT tools include databases, groupware, enterprise and web-based systems (McKinlay, 2000, cited in Kakabadse et al., 2003) as well as emails, the Internet, intranets, discussion boards, data mining tools and document and content management systems (Hendriks, 1999; Wiig, 1999, cited in Kim \& Lee, 2006). In recent years, a range of electronic collaboration tools have become available (Dalsgaard, 2006). These tools include virtual problem solving spaces, instant messaging, email applications and group support systems (Cross \& Parker, 2004), and weblogs and wikis (Dalsgaard, 2006).

Bloodgood and Salisbury (2001) argue that ICT can assist knowledge sharing, firstly, by making knowledge explicit through its codification into an information repository; secondly, by identifying individuals of specific expertise and acting as a communication tool to connect with them. Indeed, Alavi \& Leidner (2001) contend that ICT extends knowledge sharing by increasing an individual's reach.

Ackerman \& Halverson (2004) reject the notion that all individually held knowledge (i.e. tacit knowledge) can be accessed for storage in a repository, and refute the assumption that people will share that knowledge. So, while many organisations have been diligent in implementing ICT based knowledge repositories into which employees can input their knowledge, not only may these practices be ill suited to the situations for which they are used, but there may be considerable knowledge loss in the process (Huber, 2001). Repositories were often seen as a single information facility that would service an entire organisation but this was often not achievable due to a number of problems arising from political and technical difficulties. Ackerman \& Halverson (2004) also identified the inability of people to understand the material stored within the repository. Alavi \& Leidner (1999) support this finding by stating that an individual's knowledge can only be of use to another when it is communicated in a way that allows for successful interpretation and accessibility. Further, because tacit knowledge is embedded in institutional routines and is transferred directly from person to person (Blackler, 1995), it is almost impossible for it to be extracted into an explicit state, 
whereby it may be captured and codified by technology. In this respect, most ICT tools designed to facilitate knowledge sharing fail to become embedded or institutionalised within organisations (Huysman \& Wulf, 2006).

However, Huber (2001) contends that even tacit knowledge may be assisted by technology. For example, while the knowledge may not be available through a technological form, that is, a database, ICT may be the tool that locates the holder of the knowledge, for example through an expertise directory.

\subsubsection{ICT as a communication channel}

The advent of ICT increased the range of communication channels available to individuals and organisations. The richness of the knowledge sharing exchange can also be influenced by the channels through which sharing can take place (Daft \& Lengel, 1986). Rich communication channels facilitated by ICT enable face-to-face communication through a medium such as video-conferencing (Murray \& Peyrefitte, 2007). Through these channels, individuals are assisted by the presence of social cues and personalisation of use, and the ability to receive rapid feedback (Ngwenyama \& Lee, 1997, cited in Pauleen, 2003). Video-conferencing can assist individuals in sorting out complex knowledge by enabling face-to-face dialogue between individuals (Murray \& Peyrefitte, 2007). By contrast, lean communication channels include email, telephone, teleconferencing and databanks (Murray \& Peyrefitte, 2007).

Several theories have dominated the study of media choice. Early theorists, including original media richness theorists Daft \& Lengel (1986 cited in Webster \& Trevino, 1995), claim that media choice is a rational process that results from a match between the objective characteristics of a medium and the content of a message. Social influence theory places the attention on the social determinants of media choice (Fulk, Schmidtz \& Steinfield, 1990, cited in Webster \& Trevino, 1995). More recently, Miranda and Saunders (2003) have drawn on social presence theory and task closure theory to present a conceptual model that identifies information sharing as an interpretive activity (see Figure 11). 


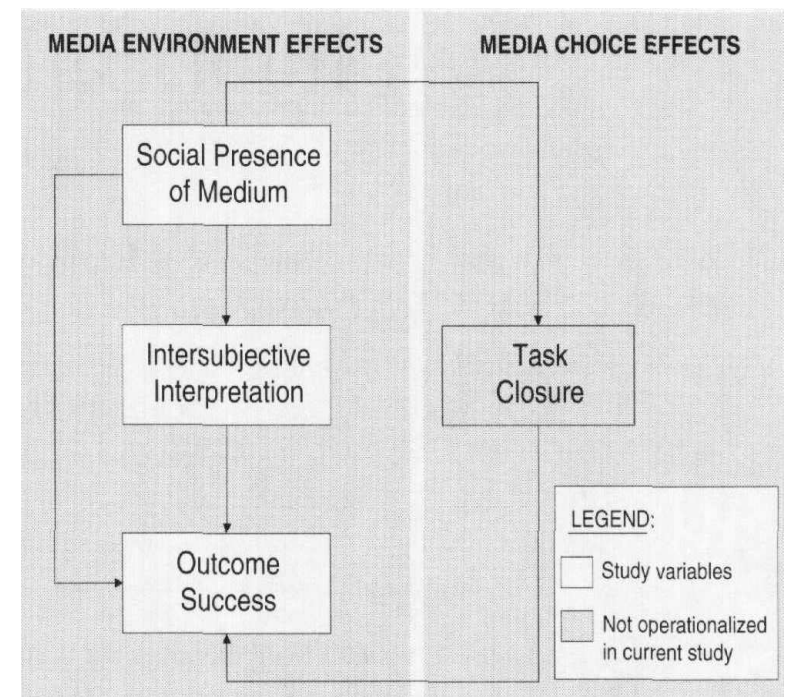

Figure 11 : Conceptual model of information sharing (Miranda and Saunders, 2003)

This model is based on the effects of the media environment in which the group is situated and the effects of media that group members actually chose for a specific communication. Social presence theory relates to the "degree to which the medium facilitates awareness of the other person and interpersonal relationships during the interaction" (Fulk et al., 1990, p. 118, cited in Miranda \& Saunders, 2003). Channels that are high in social presence include face-to-face communication and videoconferencing and are categorised by Lee \& Al-Hawamdeh (2002) as face-to-face unmediated. By contrast, electronic media and paper-based communication are described by Lee \& Al-Hawamdeh as face-to-face mediated, and are considered low in social presence. Miranda \& Saunders (2003) posit that when an individual is unavailable other than through media low in social presence, such as email, then, as task closure theory dictates, the use of this low media alternative can enable the sender to achieve closure.

\subsubsection{ICT and motivation to share}

While the primary focus of the ICT related knowledge sharing literature discusses the use of ICT as a tool for knowledge sharing, Hendriks (1999) claims that ICT may also act as an influence on an individual's motivation for knowledge sharing. Davenport (1994) challenges this assumption and posits that while ICT may provide connections that enable knowledge sharing, it does not motivate knowledge sharing behaviours.

In his research model (Figure 12), Hendriks (1999) identifies three factors on which knowledge sharing is contingent: person, context, and task. 


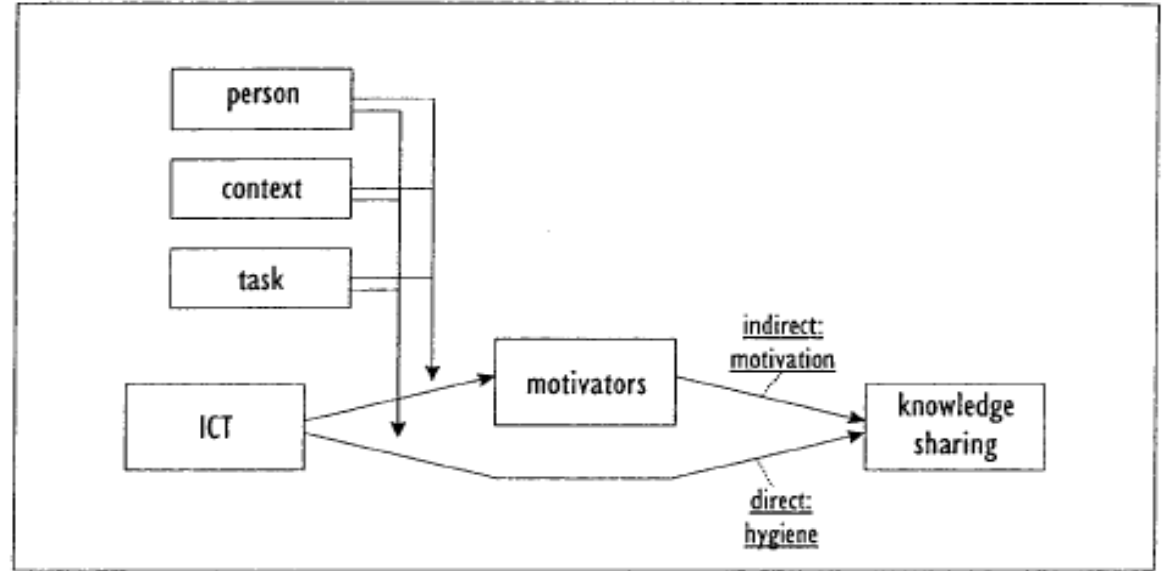

Figure 12 : Research model of the role of ICT in motivating knowledge sharing

Hendriks (1999) concludes that successful ICT applications in the field of KM must relate them to the motivations of specific workers and should reflect the particular knowledge process that the organisation is trying to achieve. Based on Herzberg's theory of motivation, Hendriks further concludes that it is the "motivation" factors rather than the "hygiene" factors which will encourage individuals to share knowledge. Therefore, it is imperative that the effectiveness of any IT implementation will occur when the individual's motivation to share is understood and the tool is developed with this is mind.

The degree to which technology is considered easy to use will influence individual's use of ICT as a knowledge sharing tool (Kim \& Lee, 2006). Ease of use of technology was first recognised through the TAM model developed by Davis, Bagozzi, \& Warshaw (1989), and extended by Venkatesh (2000). This explanatory model (see Figure 13) aimed to identify the factors that influenced the general use of ICT, and examine a range of behaviours related to ICT use (Davis, Bagozzi, \& Warshaw, 1989).

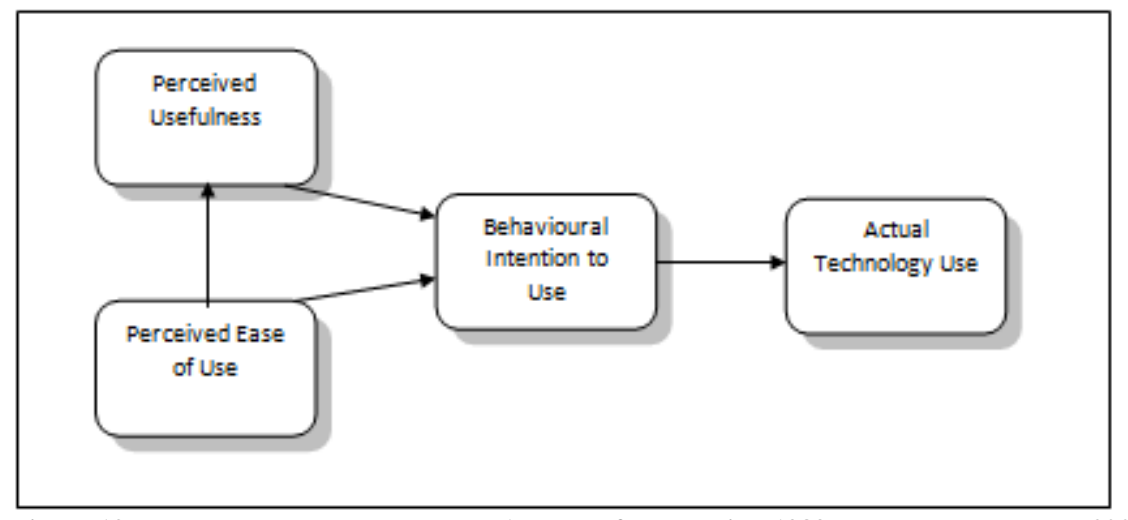

Figure 13: Technology acceptance model (adapted from Davies, 1989 and Ventakash et al., 2001) 
Perceived ease of ICT use is defined by Davis (1989) as the extent to which a person believes that using ICT will be free of effort. The perceived usefulness of ICT refers to the degree to which the use of the ICT will positively impact an individual's performance (Mathwick, Malhotra, \& Rigdon, 2001). These factors are considered to influence an individual's intention to use ICT and thus actual technology use.

In a knowledge sharing context, technology acceptance factors are considered by Hsu \& Lin (2008) in their investigation of factors that influence the use of blogs. While they found that perceived ease of use was an important factor in participation, perceived usefulness had no effect on blog usage. Similarly, Kim \& Lee (2006) found that individuals who perceived ICT systems to be user-friendly were more likely to report higher levels of knowledge sharing.

\subsubsection{Linking ICT and social capital}

While socio-technical approaches to knowledge sharing have evolved, Huysman \& Wulf (2006) believe that they still do not focus on SC as a specific requirement for knowledge sharing. Huysman \& Wulf contend that consideration of SC will contribute to the development of ICT applications that are better aligned to knowledge sharing needs, particularly in informal organisational settings such as online communities. Huber (2001) goes as far as to suggest that technology may, in fact, facilitate the development of social networks that may be used to share knowledge. Sproull \& Kiesler (1991) posit that ICT can positively affect knowledge sharing through efficiency effects, and through social effects. An efficiency effect concerns the way in which the introduction of ICT can enhance communication, which in turn, has a positive effect on the efficiency of the process by which knowledge is shared. Social effects relate to the way in which the social climate of a group is affected by the introduction of ICT, which again has an effect on knowledge sharing. In particular, the authors relate this to the development of a more collectivist norm within the group. Thus, it must be recognised that there is merit in the use of ICT and its associated tools and it can provide usefulness in relation to codification, storage, retrieval, and transfer of codified knowledge (Alavi \& Leidner, 2001; Clark \& Staunton, 1989; Kakabadse et al., 2003). 


\subsubsection{Summary of this Section}

This section introduces ICT and its role within the domain of knowledge sharing. The use of ICT as a communication channel is discussed from both a theoretical and practical perspective. The section identifies media richness theory (Daft \& Lengel, 1986); social influence theory (Fulk, Schmidtz \& Steinfield, 1990 in Webster \& Trevino, 1995); and social presence theory and task closure theory (Miranda \& Saunders, 2003), as a range of perspectives from which ICT as a communication tool has been studied.

The section describes the link between ICT and types of knowledge, and identifies issues relating to ICT and knowledge sharing. The section concludes by detailing the link between ICT and social capital.

\subsection{The public sector}

The previous sections focused on the concept of knowledge sharing and identified and discussed two distinct knowledge sharing approaches that are the focus of this study; the SC perspective, and the technological perspective. This section introduces and discusses the public sector as the specific context in which the current research will be conducted.

\subsubsection{The evolution of the public sector}

The public sector (sometimes referred to as the state sector) deals with either the production, delivery, or allocation of goods and services by and for the government or its citizens (Wikipedia, 2010).

The public administration literature details a reputed shift, over the last two decades, away from the traditional hierarchical bureaucracy toward a more commercial operational and management style (Brown, Ryan \& Parker, 2000). This new approach, known internationally as New Public Management (NPM), is portrayed as a style which will lead to a more efficient and effective public sector (Braddon \& Foster, 1996), and re-invigorate the sector to one that "is less bureaucratic, less hierarchical, and less reliant on central authority to mandate action" (Frederickson \& Smith, 2003, p. 208, cited in Hill \& Lynne, 2005). One of the key features of the transition to new public 
management necessitates a move away from the traditional hierarchical culture to one where developmental, rational, and group cultures are developed (Parker \& Bradley, 2000).

Recent analyses of NPM implementations have seen an emphasis on horizontal, hybridised, and networked aspects of governance (Kettl 2002; Salamon, 2002, cited in Hill \& Lynn, 2005). A change of this magnitude presupposes that the public sector can readily be conducted along the same lines as private industry; however confidence that this can be achieved is not shared by all (Wilson, 1994, cited in Ocampo, 1995). In their study of service delivery in the Australian public sector, Brown, Ryan \& Parker (2000) concluded that while there is scope for commercialisation to be brought into the public sector, it is necessary to redefine commercialisation to fit within the wider parameters of non-profit outcomes found in the public sector.

New Zealand is no stranger to the difficulties experienced in the public sector in other countries. Over twenty years ago, Roderick Deane, then Chairman of the New Zealand government's State Services Commission, reported that within New Zealand, "there appears to have been insufficient attention given to the potential benefits of decentralised decision making and flatter management structures. Extensive centralised rules and regulations have resulted in too many rigidities and inflexibilities" (Deane, 1986, p. 15) This statement was made two years after New Zealand commenced radical reform of its state sector (incorporating state owned entities, crown entities, and public sector organisations), through improved structures, systems, and processes aimed at achieving significant improvement of sector performance (OECD, 1999). Ensuing changes to the sector included a significant reduction in the size of the sector; the breakdown of the sector into a large number of self-contained Ministries and enterprises; commercialisation of government trading organisations; and funding reductions to organisations (Harris, 2005). One of the key features of this change was a transition from centralised regulation to decentralised management. This included the appointment of a Chief Executive Officer to each organisation, as well as a sustained effort to remove unnecessary rules and regulations from managers in an effort to make organisations more business-like, more attentive to government objectives, and more responsive to clients (OECD, 1999). 


\subsubsection{Knowledge sharing in the public sector}

One issue that is frequently debated in the literature is whether approaches that have been adopted in the private sector can be applied in the public sector context. Milner (2000) argues that while there will be some private sector organisations whose operational environments are similar to that of public sector organisations, the vast majority of private sector organisations are fundamentally different in terms of culture and outlook. Thus, despite the introduction of NPM, the private and public sectors remain fundamentally different (Cong \& Pandya, 2003). In the public sector, organisations work within government parameters, and there is the additional presence of a sector culture, as well as an individual organisation culture.

Despite researchers' predominantly private sector focus, knowledge sharing is an important issue for the public sector. As Willem \& Buelens (2007) identify, public sector organisations are knowledge intensive and require effective knowledge sharing to leverage and exploit their knowledge reserves. Yet, managing knowledge has historically been an area of difficulty for the sector (OECD, 2003). Although sharing and managing knowledge is not new, the public sector has been slow to realize its importance (Bundren, 2010; Taylor \& Wright, 2004). Consequently, Bate \& Robert (2002) report that there is little published public sector based knowledge management research. Of the limited research that has been conducted in the public sector context, most has been conducted within the confines of single organisations, and the primary focus has been on the need to measure and manage explicit and existing knowledge, rather than tacit knowledge sharing and development (Jorgensen, 2004). This preference may be the product of the hierarchical structures of the public service and its focus on the processing of information (Takeuchi, 1998). As a consequence, this focus has seen the sector invest heavily in ICT as a KM tool. In practice, Hackett (2000) suggests that more effective knowledge practices are to be attained through informal employee networks and modified workplace practices. Kleiner (1995, cited in Jorgensen, 2004) argues that tacit knowledge will be revealed through collaboration that is based on trust, openness, and reciprocity. Indeed, individuals will more effectively manage knowledge where human and SC have become fundamental principles of the organisation (Gavigan, Ottitsch, \& Mahroum, 1999). Thus, public sector organisations should advocate social and relationship skill building between staff (OECD, 2001). 
In addition to public sector organisations sharing knowledge internally, there is considerable pressure on organisations to work collaboratively across institutional boundaries. The drive for greater inter-organisational collaboration has been largely driven by the continued evolution of technology and the development of national knowledge economies. Indeed, public sector organisations play a key role in facilitating the economic, cultural, and technological conditions conducive for the development of knowledge economies (Hearn \& Rooney, 2002). In doing so, new challenges arise that cut across policy and services areas and cannot be solved by the single-organisation or silo approach (Mitchell \& Shortell, 2000; Pearson, 1999; Rhodes, 1998; Waddock, 1991, cited in Keast, Mandell, Myrna, Brown \& Woolcock, 2004). They require new approaches that see individual organisations working co-operatively and cohesively as a unified sector where knowledge sharing both within and between organisations is the norm. Bundren (2010) asserts that the radical improvement of public services requires the equally radical improvement of the management of knowledge across the sector. Yet, despite acknowledgement of the growing importance of inter-organisational knowledge sharing, research that explores the dynamics of knowledge sharing in this context remains limited (Mulgan, 2005; Tang, 2008).

\section{Joined-up government}

The drive for greater collaboration has been formalised through the concept of Joined Up Government (JUG). This concept proposes a move away from public sector organisations operating as silos, towards a co-ordinated sector where knowledge sharing between organisations is actively supported. Lips (2008) identified the benefits of JUG as the provision of better service to citizens; better coordination in government; more cost efficient work through sharing of resources and reduced duplication of effort and output; and, innovation through new ways of working. Johnson (2005) sums up three types of joined up arrangements:

1. Whole of Government Integration: characterised by a top down whole of government policy framework based on what government seeks to achieve followed by practical strategies to achieve whole of government integration

2. Service Delivery Integration: characterised by the collection together of information and services about a shared customer or common issue 
3. Integration around programmes: ongoing cooperation and collaboration by a community of problem solvers.

Therefore at an operational level, collaboration can be facilitated through a spectrum of approaches ranging from ongoing partnerships between organisations through to the formation of designated inter-organisational project teams. Traditionally, projects have been defined as unique tasks with predetermined start and end dates, a specified goal or outcomes, and a number of different activities (Packendorff, 1995 in Lofstrom, 2010). However, as Lofstrom (2010) points out projects in the public sector can differ from this traditional perspective, where they are commonly given identities, hierarchies and, in some instances, a distinct location (Lofstrom, 2010).

\subsubsection{Barriers to knowledge sharing in the public sector}

Taylor and Wright (2004) identify four challenges to knowledge sharing in the public sector context. Firstly, the inherent rule-based culture does not promote innovation or improvement, but rather seeks compliance; secondly, the sector is dominated by media and public scrutiny; thirdly, the nature of government policy imposes change on the sector and is often viewed as interference; and, lastly, the need for inter-organisational collaboration is at odds with the sector's focus on individual organisation performance.

Liebowitz and Chen (2003, cited in Holsapple, 2004) found that the bureaucratic nature of organisations and their accompanying hierarchies can hinder knowledge sharing in the public sector. In the traditional hierarchical model, decision making and information flow up and down the hierarchy, but not across organisations. This can be detrimental to an organisation and can significantly slow organisational processes (Huczynski, 1989), as well as adversely affect staff development and organisational growth (Banks \& Powell, 2002). Mintzberg (1978) identifies this type of mechanistic bureaucracy as more common in government organisations, and states it is characterised by the presence of standardised processes that guide the undertaking of tasks within the organisation. By contrast, an adhocracy is commonly seen as the opposite of the classic bureaucracy. In this model, the organisational structure is based on decentralised decision making and absence of formal rules and procedures, and is one which facilitates an intensity of knowledge work. Although the OECD (2003) states that there are no apparent moves towards the implementation of an adhocracy, they note that 
almost seventy-five percent of public sector organisations surveyed indicated that over the last five years they had taken initiatives to decentralise and delegate authority to lower hierarchical levels and create internal networks to share information. Indeed, networks and network structures are increasingly being seen as pivotal tools to develop innovative ways to tackle problems confronting the public sector (Keast, Mandell, Brown \& Woolcock, 2004).

In relation to the public sector, O'Toole (1997) defines networks as a pattern of two or more units, in which not all major components are encompassed within a single hierarchical array. There are several ways in which arrays may be joined; for example, they may include some combination of organisations (or parts of organisations) of the same government, links among units of different governments, ties between public organisations and for-profit companies, and public/nonprofit connections, as well as more complex arrangements including multiple types of connections in a larger pattern. Within these networks, staff may become connected with other actors outside of their own distinct hierarchy or organisation and successful interaction may be critical to success of the collaborative effort. O'Toole \& Meier (1997) present an in-depth study on the role of networks within the public sector and argue that despite the view of public sector organisations as hierarchical public bureaucracy, an increasing number of scholars are arguing that public management often takes place in and on networks of actors (O’Toole \& Meier, 2004).

While the barriers to knowledge sharing are distributed across social, cultural, organisational and technical dimensions, the predominant approach to facilitating knowledge sharing in the public sector has been technology focused. However, there is evidence of a shift to adopt broader approaches. For example, the Australian government has acknowledged that the provision of a technical and social infrastructure for collaboration and knowledge sharing would assist the development of collaborative knowledge networks that could improve networked government by:

- Helping to transfer best practices throughout the network

- Developing new knowledge and enhance learning

- Fleshing out solutions to daily problems

- Reducing misunderstanding and building trust between network partners 
- Helping the network partners learn from each other's successes and, more importantly, from their mistakes

- Helping government to better integrate and align the efforts of their partners with their strategic objectives (Goldsmith \& Eggars, 2004 p.16).

\subsubsection{Knowledge sharing in the New Zealand public sector ${ }^{3}$}

In New Zealand, acknowledgement of the need to collaborate and share information and knowledge across organisations has spawned an increasing level of inter-organisational collaboration (Walker, 2004). Three of the most significant initiatives to address the need for better inter-organisational collaboration and sharing are "Pathfinder", "Review of the Centre", and, most recently, the development of six key development goals that the government proposes will provide a system of world class State Services to serve both the needs of government and of New Zealanders (SSC, 2006).

\section{Pathfinder}

The purpose of the 2001 Pathfinder Project was to develop practical ways of improving results for departments across New Zealand's central government (Baehler, 2003), and to develop mechanisms for successful planning and management based on outcomes. It identified a number of factors necessary for successful collaboration within the sector, and a number of obstacles that should be avoided (see Table 11).

\footnotetext{
${ }^{3}$ Section 2.6.4 provides a synopsis of knowledge sharing in the New Zealand context. The majority of the content has been derived from information publicly available from organisational websites and is supplemented by research literature sources. As such, it does not constitute a formal review of the literature but has been included for its relevance to the specific context of the study.
} 
Table 11: Factors for successful collaboration (Pathfinder, 2001)

\begin{tabular}{|l|}
\hline Success factors \\
\hline $\begin{array}{l}\text { Governance structure encouraging co-production and balancing central organisation commitment with } \\
\text { department ownership of results }\end{array}$ \\
\hline Dedicated staff with high levels of experience and expertise \\
\hline Firm commitment of dedicated resources, including time \\
\hline Firm commitment from participants to contribute substantive examples and material to the project stocks \\
\hline $\begin{array}{l}\text { Participants must be actively engaged in the business at hand but also have a broader view of their } \\
\text { departments and of the sector in general }\end{array}$ \\
\hline Diversity of participants \\
\hline Establishment of ground rules for group interaction and protocols for managing conflict and disagreement \\
\hline Clear expectations of an extensive draft-comment-redraft cycle of working \\
\hline Strong participant commitment to providing feedback on material and introducing new material \\
\hline $\begin{array}{l}\text { A project management style that keeps participants focused on tasks but caters for diverse approaches to } \\
\text { undertaking those tasks }\end{array}$ \\
\hline Clarity around the model of collaboration being embraced \\
\hline Adequate timing to conduct the project \\
\hline Correct scale with eight organisations as a maximum \\
\hline Obstacles \\
\hline Endemic suspicion of central organisations intentions to assist versus assess \\
\hline Departmental political agendas \\
\hline Departmental resource constraints \\
\hline Language differences departments, sectors and functions \\
\hline $\begin{array}{l}\text { Fundamental disagreements about the feasibility of measuring government's impact in certain areas of } \\
\text { policy }\end{array}$ \\
\hline Fundamental differences in the types of business conducted in the public sector. \\
\hline Fundamental differences across professions in their approaches to outcomes \\
\hline The "eureka" all-at-once nature of learning \\
\hline
\end{tabular}

\section{Review of the Centre}

Review of the Centre aimed to determine how well the public management system responded to the needs and expectations of New Zealand ministers and citizens. The project delivered a number of key findings including the need for integrated service delivery and attacking fragmentation within the sector. As a result, the government instigated the Integrated Service Delivery: Regional Co-ordination Workstream, with a mandate to determine how government organisations could work better together. While these reviews identified specific conditions required for collaboration, they did not identify any requirements that specifically relate to knowledge sharing.

\section{Key development goals}

In 2005, the New Zealand government revisited the need for better inter-organisational collaboration and began development of six development goals. The goal of "Coordinated State Services" aimed to support New Zealand public sector organisations to work together to achieve defined outcomes by sharing information, resources, and responsibilities (SSC, 2008). To clarify the use of the term coordination rather than collaboration, the SSC states: 
In a State Services context, coordination means the sharing of information, resources and responsibilities to achieve a particular outcome. Organisations coordinating can mean that they elect to share decision-making authority, provided existing accountabilities are not modified... In contrast, collaboration refers to arrangements that encourage joint decision-making with regard to directionsetting, planning, implementation and review. It is more than just the sharing of work; it is the sharing of responsibilities and, in some instances, of mandated authority. Therefore, it usually entails Ministerial involvement. (SSC, 2008).

The SSC purports one of the benefits of co-ordination to be the ability to exploit opportunities such as the sharing of data and information (SSC, 2008). The main output the work undertaken in respect of the Co-ordinated State Services goal has been the development of a three dimensional framework identifying nine success factors for successful co-ordination (see Table 12 below).

Table 12: Factors for Success Co-ordination (SSC, 2008)

\begin{tabular}{|l|l|}
\hline Dimension & Factors \\
\hline Mandate & Leadership Commitment \\
\hline & Ministers' and stakeholders' buy in \\
\hline & Defined and agreed joint outcomes \\
\hline Systems & $\begin{array}{l}\text { Appropriate and documented governance and accountability } \\
\text { frameworks }\end{array}$ \\
\hline & Sufficient and appropriate resources \\
\hline Behaviours & Process to measure performance form established baselines \\
\hline & Right representation, skills, and team leadership \\
\hline & Organisational cultures that support co-ordination \\
\hline & Shared culture, language, and values \\
\hline
\end{tabular}

A comparative analysis of the factors outlined in Table 12 above and those originating from the findings of the Pathfinder Project (see Table 11) clearly shows areas of consistency between the recommendations. For example, both projects recognise the importance of agreed outcomes, governance, appropriate resources, and people related factors such as skills and expertise, as well as shared culture, language, and values. However, while this consistency can be viewed positively, and as encouraging in terms of its closer alignment with recommendations from the knowledge sharing literature, it also suggests that relatively little has changed within the sector during the time that has elapsed since the release of the "Pathfinder" recommendations in 2001 and the development of the "Factors for Successful Co-ordination" in 2008. The analysis of the findings from the two projects highlights the gap between the recommendations from 
these two projects, and the predominantly ICT-based approach adopted by the public sector.

The drive to share knowledge within and across public sector agencies has been centred on the New Zealand e-government strategy. First developed in 2000, the strategy contained several initiatives aimed at improving sharing across organisations in the sector including the development of a new high-speed network, the Government Shared Network (GSN), which would enable information to be shared more quickly and effectively by all government organisations.

A further e-govt initiative, the Public Sector Intranet (PSI) aimed to provide public servants with a single point to share and find information. The goals of the PSI were somewhat broader than simple information finding and the New Zealand e-govt website discerns that the PSI "enables a sense of community, shared interests, and crossorganisation cooperation" (E-Government, 2006a).

Other initiatives included the development of shared workspaces designed specifically to support collaboration. Similarly to the GSN, the use of shared workspace was not compulsory, and individual public sector organisations were still able to implement individual organisational workspaces. The government's business case to support shared workspaces identified the primary goal as supporting project work and policy development between organisations and stakeholders (SSC, 2003). Shared workspaces provide online tools to enable information sharing and working between government organisations, including access to meeting and events calendars, document sharing, notifications and discussions, announcements, and discussion lists. Shared tools such as these were seen as a specific aid to cross-organisational collaboration (see Figure 14). 


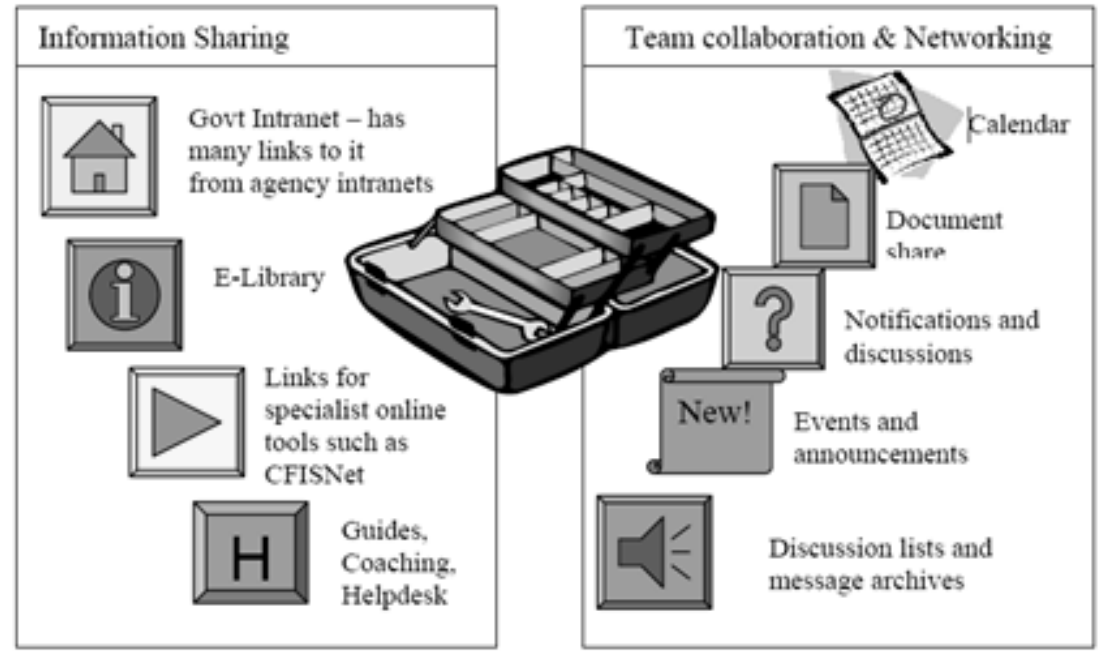

Figure 14: Toolbox Vision of Shared Workspace (SSC, 2003)

Social networking tools - for example, weblogs, wikis, online forums - are also being considered and used in some New Zealand government organisations; these include use in a number of cross-government initiatives (E-government, 2009). While these tools are seen as useful in supporting knowledge sharing, there is still concern about the use of external social networking tools such as MySpace, FaceBook, and Flickr and these have not yet been approved for use within public sector organisations.

\subsubsection{Summary of this section}

This section introduced the public sector as the specific context for this study. The section began with a brief overview of the shift from the traditionally highly centralised and bureaucratic structure and approach of the public sector, to one that is more commercial and cohesive in approach. The section continued by detailing the approaches and barriers to knowledge sharing in the public sector, and identifies the need for more research to be undertaken within the public sector context. The section concludes with an overview of knowledge sharing in the New Zealand public sector.

\subsection{The conceptual framework}

\subsubsection{The purpose of a conceptual framework}

Research can be conducted without the development of an explicit conceptual framework (Creswell, 2003). However, the development of such a framework can serve the researcher in multiple ways. A conceptual framework can be used to explain, "either graphically or in narrative form, the main things to be studied - the key factors, 
constructs or variables - and the presumed relationships among them" (Miles and Huberman, 1994, p.18). A framework can enable the researcher to organise ideas and concepts into a coherent manner that makes them easy to communicate to others. Frameworks can also be used as an explanation for behaviour and attitudes, or to provide an underlying theoretical lens to guide the study.

Miles \& Huberman (1994) state that the amount of initial structure used to guide research is an important issue. They suggest that there is benefit in developing a framework at the outset of a research study as it may particularly assist less experienced researchers to more clearly focus on the study's key issues. It is likely that the framework will develop in line with the direction and findings of the study as it progresses. Miles \& Huberman (1994) determine that a conceptual framework may be viewed as a "researcher's first cut at making some explicit theoretical statements" (p. 91).

The major focus of this research is the exploration of knowledge sharing, and the way that knowledge is shared in inter-organisational collaborative endeavours in the New Zealand public sector. In this study, the conceptual framework is used to draw together the researcher's thoughts about the phenomenon of knowledge sharing, and to connect these to the key themes of interest from the literature review. This initial conceptual framework will help to guide the research process. At the conclusion of the research process, the conceptual model will be reviewed and revised to reflect the specific findings of the study.

\subsubsection{Development of the conceptual framework in this study}

The conceptual framework encapsulates the main concepts drawn from the literature and identifies the potential relationships between these concepts (see Figure 15).

The framework posits that, based on the extant literature, both SC and ICT play a role in the way in which knowledge is shared within inter-organisational teams. The framework also identifies a possible relationship between SC and ICT. This relationship has been alluded to within the literature review, but has not been adequately understood due to the lack of studies that have integrated the technical and the social dimensions ( $\mathrm{Fu} \& \mathrm{Lee}, 2005$ ). This research aims to determine the nature of those roles within the context of the study, and to provide empirical evidence to support the claims. 


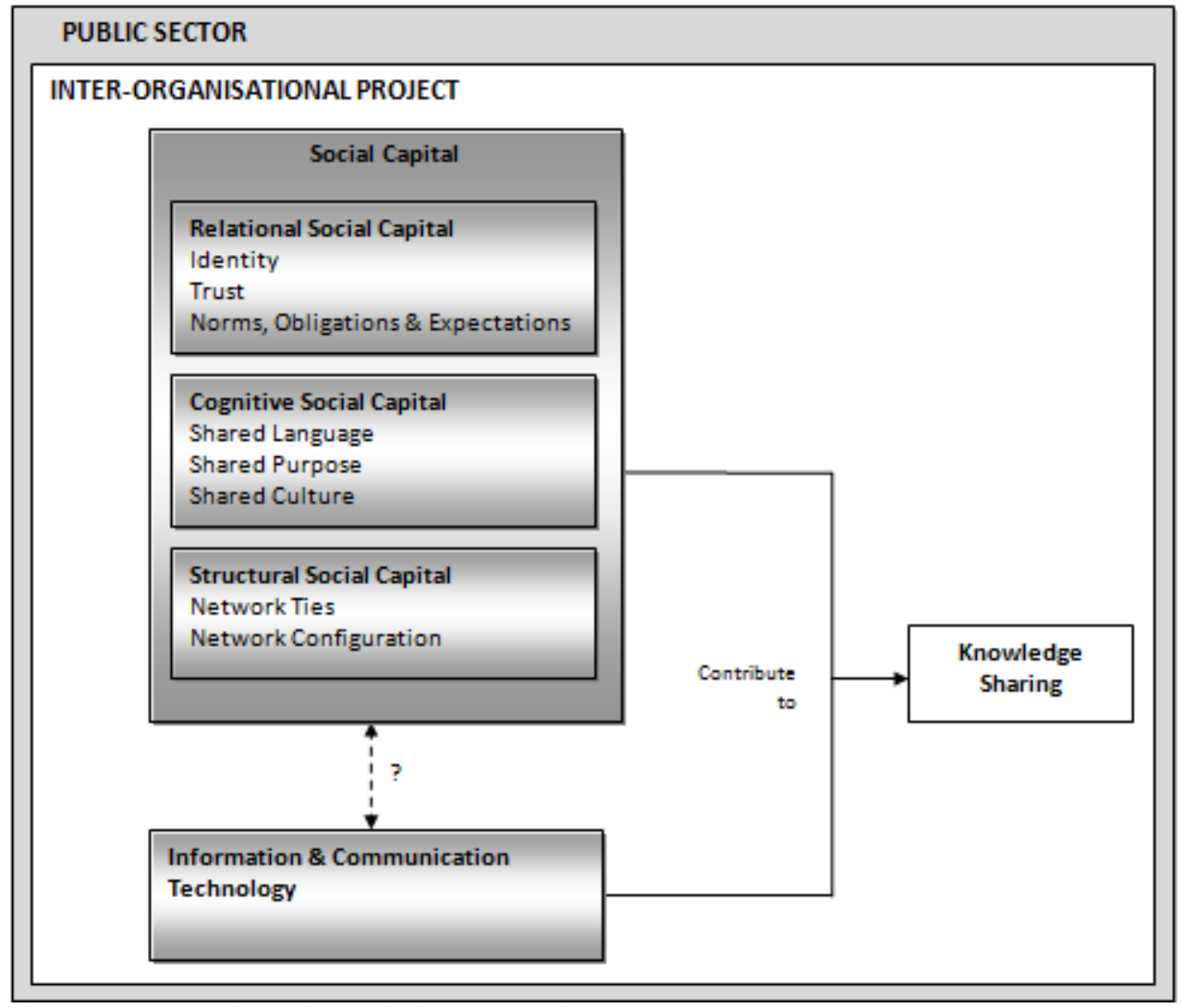

Figure 15: Conceptual Knowledge Sharing Framework

The framework identifies the public sector as the over-arching context for the study. The review of knowledge sharing in the public sector (see Section 2.6) suggests that the mechanistic nature of the sector in terms of bureaucratic process and multi-layered hierarchy is one that is not conducive to knowledge sharing. Consequently, knowledge sharing has historically proved to be difficult to undertake within the sector.

The boundaries of the study are further distinguished by the identification of public sector inter-organisational collaboration as the specific domain of interest. Collaboration denotes specific initiatives undertaken between two or more public sector organisations, and represents a structure through which inter-organisational knowledge sharing can take place. Inter-organisational teams are characterised by the multiple perspectives, beliefs, values, and cultures that individuals from different organisations bring to the collaboration and, as such, are more complex than sharing within a single organisation or business unit. While the literature shows that instances of interorganisational collaboration are increasing (Walker, 2004), surprisingly little research attention has focused on inter-organisational knowledge sharing within the sector (Bundren, 2010; Taylor \& Wright, 2004). 
As illustrated in the literature review (Section 2.5), early studies identified ICT as an important consideration of knowledge sharing. In later studies, the role of ICT has been questioned and many scholars have come to recognise the role of ICT as an enabler and supporter of knowledge sharing, rather than a driver. Despite this recognition, many organisations continue to direct significant financial resources into ICT infrastructure and tools in a bid to drive knowledge sharing. The literature review also notes that, in the NZ public sector, ICT continues to be a key consideration in the government's approach to knowledge sharing, particularly in regard to supporting interorganisational sharing.

The more recent knowledge sharing literature focuses on understanding how SC, and specific aspects of SC - for example, trust - influences knowledge sharing (see Section 2.4). A common approach to studies that explore SC and its relationship to knowledge sharing has been to focus on one or more of SC's three key dimensions espoused by Nahapiet \& Ghoshal (1998); that is, relational, cognitive, and structural SC. A predominant theme of the literature posits that networks facilitated by SC will be more enduring than formally structured teams, such as organisational teams (Huysman \& Wulf, 2004). Though research evidence identifies SC as a key influencer of knowledge sharing, there has been little research that explores knowledge sharing in the public sector context.

The researcher also acknowledges that other factors such as leadership, work management practices (Smith \& McKeen, 2003), and team design (Hoegl et al., 2003), leadership and organisational support (Fedor et al., 2003) have been identified as influential in the practice of knowledge sharing. These factors have been included in the literature review to indicate that they are considered important to the field of knowledge sharing, and to ensure a thorough review of the literature was undertaken. However, they are considered to be outside the main focus of this research and as such are omitted from the conceptual framework.

\subsubsection{Confirmation of the research questions}

The understandings gained from the literature review confirmed the need for empirical research that explores knowledge sharing in the public sector, and supported the development of the formal research questions. 
The primary focus of the research is to better understand the phenomenon of knowledge sharing in the context of inter-organisational collaboration in the New Zealand public sector, and is encompassed in the primary research question:

\section{Research Question One: How is knowledge shared in inter-organisational collaborations in the public sector?}

The first research question seeks to develop a broad understanding of how knowledge is shared in inter-organisational teams, and to identify the factors that play a role in that knowledge sharing. The research will seek to answer the question by providing a comprehensive understanding of the underlying characteristics of each of the cases, for instance the formal structure of the teams, and any processes or procedures that are in place to guide the work undertaken by the team. These characteristics will define the "formal" team structure defined by Zack (2000) as "who reports to whom", and will provide a basis on which to compare the findings of the structural SC data that represents "who shares with whom."

The research will also explore the range of knowledge sharing activities that are available within each of the Cases and investigate how and why these activities are utilised.

The primary research question is supported by two secondary questions that seek to provide a more in-depth understanding of the two predominant knowledge sharing approaches identified in the literature.

\section{Research Question Two: What is the role of social capital in inter-organisational knowledge sharing in the public sector?}

The research will seek to answer question two by identifying the specific role of SC in inter-organisational knowledge sharing. It focuses on drilling down into the research findings to identify how particular aspects of the relational, cognitive, and structural SC dimensions manifest within each case, and will look to identify the role of these factors in relation to knowledge sharing. In addressing this question, the research will also explore relationships within and between the dimensions within each case, and compare these roles across the cases. 
The social network data derived from the structural SC dimension will identify interactions and ties between participants, and the flow of knowledge within each case. This data represents "who interacts with and shares information with whom", referred to as the "informal structure" (Zack, 2000), and will enable the research to compare the formal and informal team structures.

Research Question Three: What is the role of ICT in inter-organisational knowledge sharing in the public sector?

The third research question focuses on the role of ICT within each case. It requires exploration of how ICT manifests within the inter-organisational teams and the way in which it supports knowledge sharing between team members. This research seeks to identify the availability and use of ICT within each Case, as well as individuals' perceptions and use of ICT. These findings will be discussed in the context of the government's commitment to ICT as a knowledge sharing tools approach. The research will also explore any potential relationship between ICT and SC.

\subsection{Summary of Chapter 2}

The chapter has identified and summarised the key aspects of the literature relating to this research, and presented a conceptual model of the research factors to be explored in this study.

The first section of the review introduced the philosophical foundations of knowledge and presented a selection of the many knowledge definitions and typologies. The concept of $\mathrm{KM}$ is presented incorporating an overview of the various approaches and frameworks that have been developed to guide scholars and practitioners in KM research and practice. The section continued by identifying the key knowledge processes as determined by a range of scholars. Of these processes, knowledge sharing forms the basis of this research and further elucidation was provided through a more indepth view of this single process. The review has presented several important approaches to knowledge sharing and discussed a range of factors that have been identified by scholars as pertinent to the knowledge sharing domain.

The concept of SC is introduced and discussed in relation to its theoretical application to knowledge sharing. The relational, cognitive, and structural dimensions are identified 
as those most often explored in studies and knowledge sharing, and specific factors within these dimensions are identified and discussed. Social networks are introduced as a key theme of interest to research that investigates knowledge sharing and SC, and several approaches to network types have been presented.

The review continues with a section discussing the role of ICT in sharing knowledge. It identifies the predominant ICT tools that have been considered and factors that impact on the use of them - for example, perceived ease of use and perceived usefulness. This section also presents the role of ICT as a communication channel.

The final section of the literature review presents the public sector as the specific context in which this research is focused. A brief history of the evolution of the sector is presented and the importance of knowledge sharing discussed, with specific reference to the concept of Joined Up Government and its requirement for public sector organisations to collaborate and share knowledge.

The literature review provides the key points of focus pertaining to this research and presents evidence of the three literature gaps identified in Section 1.1 of the thesis introduction. It identifies the need for a greater research focus on knowledge sharing practices within the public sector (Bate \& Robert, 2002; Bundred 2010; Jorgensen, 2004; Taylor \& Wright, 2004). This need is particularly important at the interorganisational level where only limited research exists (Bundred, 2010; Mulgan, 2005; Tang, 2008). The review also shows that, despite a greater emphasis on the SC aspects of knowledge sharing in the private sector, this remains a concept that the public sector is yet to explore (Gavigan, Ottitsch, \& Mahroum, 1999; Jorgensen, 2004; OECD, 2001; Takeuchi, 1998). While the review has confirmed the lack of any one dominant theoretical approach to knowledge sharing, it discusses the contributions that have been made through both social network theory and various communication theories including media richness theory, social influence theory, and, more recently, social presence theory and task closure theory.

The final section of this chapter presents a conceptual framework that encapsulates the key factors of interest to this study, and identifies the potential relationships between these factors. The framework has enabled the researcher to identify the main themes of 
the research, posit high-level relationships between the themes, and distinguish the boundaries of the research. 


\section{THE RESEARCH PROCESS}

The previous chapter presented the literature review and conceptual model that guides this study. A range of factors from the knowledge sharing literature were identified and considered in respect to the objectives of this research. Selected factors were then conceptualised into a framework.

This chapter guides the reader through the research methodology and design. The chapter is organised into four main sections:

- Section 1: Summarises three philosophical approaches to research, and identifies and justifies the approach adopted in this study.

- Section 2: Describes the research methodology adopted for this research and discusses specific considerations of the approach selected.

- Section 3: Describes the mixed methods approach, and the specific mixed methods approach adopted within this study. The unit of analysis for the study is presented. An overview of SNA and its use as a research method is also provided. The section concludes by describing how issues related to the validity of qualitative data are addressed within the study.

- Section 4: Details the specific processes and procedures undertaken during data collection and analysis.

\subsection{Philosophical paradigms in research}

The researcher's choice of paradigm, or worldview, is critical to the conduct and the outcomes of the research study. Bassey (1990, p. 37) defines a paradigm as "a network of coherent ideas about the nature of the world and the functions of researchers which, adhered to by a group of researchers, conditions their thinking and underpins their research actions." Paradigms are worldviews or belief systems that are a reflection of and guide the decisions that researchers make (Tashakkori and Teddlie, 1998). The underlying research paradigm reflects the epistemological and ontological beliefs of the researcher and directly influences the study and its findings. For the novice researcher, determining an epistemological stance can be one of the most difficult, but critical, research decisions that must be made. 
Guba and Lincoln (1994) posit that paradigms consist of three dimensions that respectively seek to answer the ontological, epistemological, and methodical questions:

1. What is the form and nature of reality?

2. What is the relationship between the researcher and what can be known?

3. How can the researcher find out if whatever they believe can be known?

The researcher's ontological position relates to "that which can be known". In philosophy, ontology is often referred to as metaphysics, the discipline of inquiry into the most basic and general features of reality such as the nature of existence and identity (Bera \& Wand, 2004). Ontology describes the reality that a scientist/scholar holds to exist, and can be exemplified by questions akin to "What exists", "What am I?".

Epistemology refers to our theory of knowledge; more specifically how we acquire knowledge. The philosopher Socrates determined that knowledge occurs when true belief is accompanied by rational account, thus it must be accompanied by propositions or statements that justify the certainty of the belief. Hirscheim (1985, cited in Mumford, Hirschheim, Fitzgerald, \& Wood-Harper, 1985) states that knowledge is therefore fallible and conditional and that, in any society, knowledge claims are an agreed best understanding at a particular point in time. These claims may, in the future, become invalid when additional information is made available. Therefore one's epistemological stance refers to the "nature of the relationship between the knower and the would-be knower" (Guba \& Lincoln, 1994, p. 108), and should support and reflect the ideological position of the researcher.

The methodological dimension relates to the way in which the researcher pursues the area of interest, or as Creswell (2003) describes, the processes for studying a given phenomenon.

To elucidate scholars' differing perspectives, a number of frameworks or typologies have been introduced. One of the most prominent and widely cited is that of Burrell \& Morgan (1979 cited in Clegg, Hardy \& Nord, 1996) who follow Kuhn's concept that each paradigm is indeed mutually exclusive, and that researchers must adopt an individual paradigm to guide their work. Their model posits four exclusive paradigms: 
functionalism, interpretivism, radical structuralism, and radical humanism (see Figure 16).

The regulation quadrant is dominated by the status quo and social order and is the natural home of the interpretivist and functionalist paradigms. These two paradigms are clearly exclusive of one another and this is denoted by the clear delineation of their subjective or objective natures. By contrast, the upper two paradigms relate to radical change as denoted by first order change, structural conflict or modes of domination.

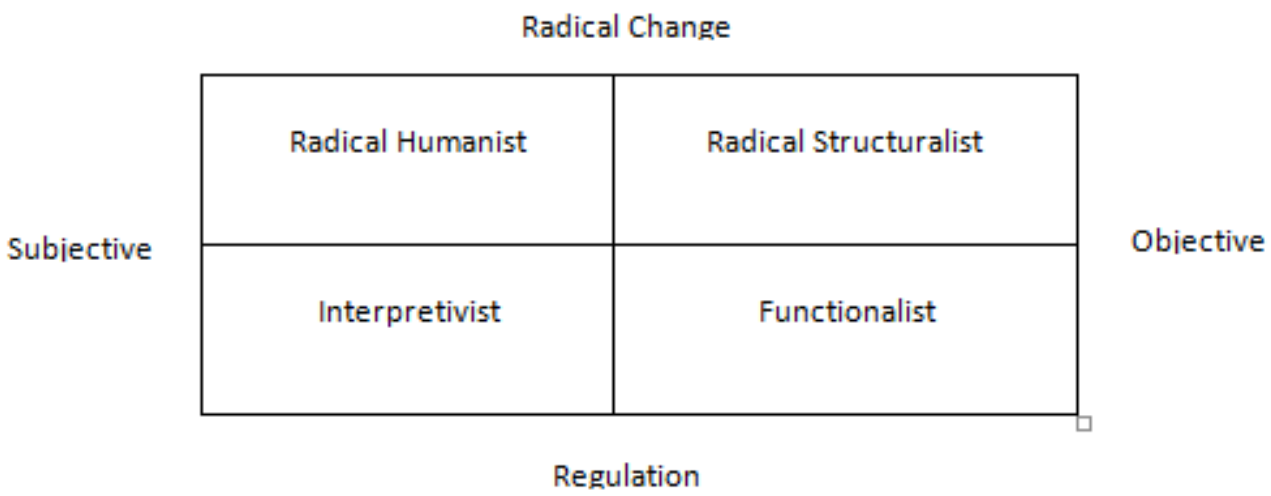

Figure 16: The four paradigms (adapted from Burrell \& Morgan, 1979)

The strict exclusivity of the Burrell \& Morgan framework opposes the concept of pluralism, whereby a simultaneous application of multiple paradigms is accepted. This dissatisfaction has provoked much discussion within the Information Systems (IS) literature (Walsham, 1995), and, as a result, several different perspectives have emerged. Guba \& Lincoln (1994) suggest four underlying research paradigms: positivism, post-positivism, critical theory, and constructivism. Orlikowski \& Baroudi (1991) posit the three dimensions of positivism, interpretative, and critical science. Creswell (2003) puts forward four paradigms or worldviews: post-positivism; constructivism; advocacy/participatory, and pragmatism. In their review of philosophical paradigms, McKenzie \& Knipe (2006) draw from Creswell (2003) and Mertens (2005) to develop an overview of the main concepts and languages associated with each of the paradigms as shown in Figure 17. 


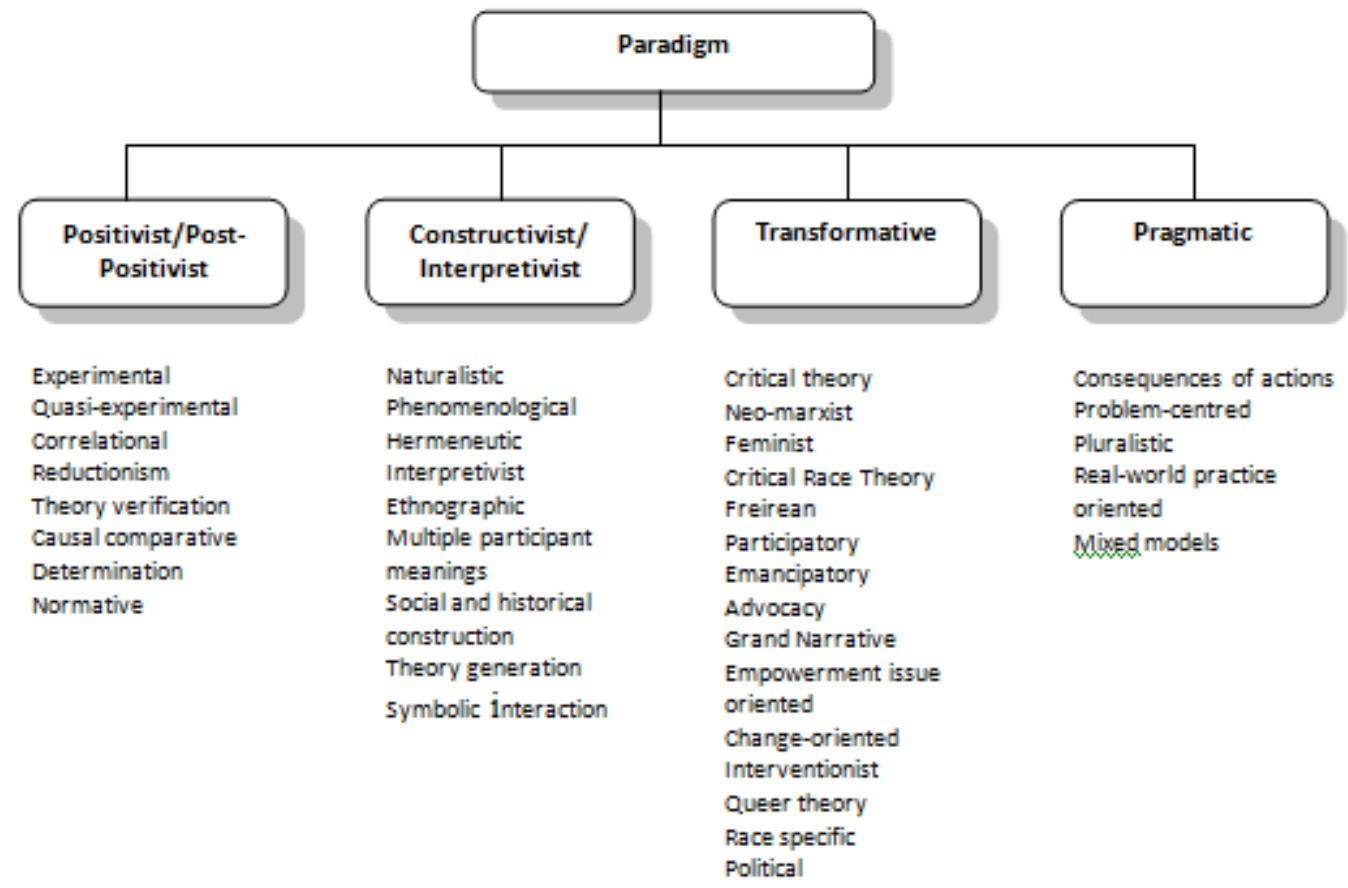

Figure 17: Paradigms: Language commonly associated with major research paradigms (adapted from McKenzie \& Snipe, 2006)

\subsubsection{Positivist and post-positivist paradigms}

\section{Positivism}

The positivist perspective is defined by Burrell and Morgan (1979) as an epistemology which seeks to explain and predict what happens in the social world by searching for regularities and causal relationships between its constituent elements, and which, from an ontological viewpoint, adopts a position of realism, whereby the universe is comprised of objectively given, immutable objects and structures that exist as empirical entities, independent of the observer (Hirschheim \& Klein, 1989). Epistemologically, positivists are concerned with the hypothetic-deductive testability of theories; ontologically, reality exists objectively and independently from human experiences; and methodologically research must be conducted by a value-free method (Chen \& Hirschheim, 2004). Mingers (2001) reported that positivist studies are more likely to collect data through passive observation, measurement/statistical analysis, survey/questionnaire, experiment, simulation, and case study. IS research can be classified as positivist if the research has evidence of formal propositions, involves the quantifiable use of variables, and seeks to test hypotheses and/or draw inferences from a sample to general population (Orlikowski \& Baroudi, 1991, p. 5). 
Post-positivism

Post-positivism challenges the concept of positivism and suggests that when studying human behaviour and actions, researchers cannot be certain about claims of knowledge (Creswell, 2003). Post-positivist researchers recognise limitations within the positivist stance, and talk about probability rather than certainty (Crotty, 1998). In the postpositivism paradigm, researchers must be able to see the whole picture, and rely not just on facts but also on the context within which those facts occur (Ryan, 2006). So, while post-positivist researchers continue to focus on reductionism, they also take into account a broader picture than that of the traditional positivist.

According to (Richie \& Rigano, 2001, in Ryan, 2006), post-positivist researchers consider that truth is constructed through a dialogue from which conflicting interpretations may be raised and discussed amongst the members of a community. Further, they claim that the purpose of data gathering is to talk about the issues raised, to consider the reactions of participants, and develop an understanding of the way in which these issues are interwoven.

\subsubsection{Constructivist and interpretivist paradigms}

Creswell (2003) purports that in the paradigm of social constructivism, individuals develop subjective meanings of their experiences in the world that are both varied and multiple. Culture and context are important issues in social constructivism that enable the researcher to understand and construct knowledge based on this understanding (Derry, 1999; McMahon, 1997 in Kim, 2001). Creswell (2003) suggests that individuals develop these subjective meanings through interaction with others, as well as through an individual's historical and cultural norms.

Socially constructed knowledge claims are often combined with the interpretative paradigm (Creswell, 2003) in which the social context is identified as critical in understanding the phenomena under investigation. Within the context of Burrell and Morgan's typology (1979), the interpretative paradigm can be seen to reflect a subjective ontology. Epistemologically, interpretivist research focuses on the full complexity of human sense making as the situation emerges (Kaplan and Maxwell, 1994), and allows participants to use their own words and images, and to draw on their own experiences and beliefs. Ontologically, it is the construction and reconstruction of 
human and social interaction that gives the subjective meaning to reality (Chen \& Hirscheim, 2004). In this way, the interpretive researcher attempts to gain a deep understanding of the phenomena being investigated, and acknowledges their own subjectivity as a part of this process (Darke, Shanks, \& Broadbent, 1998).

\subsubsection{Transformative paradigm}

McKenzie and Knipe (2006) draw together the perspectives of critical research (Guba \& Lincoln, 1994; Orlikowski \& Baroudi, 1991) and the advocacy/participatory worldview (Creswell, 2006) and encapsulate these within the "transformative" paradigm.

Critical research perspectives are reflected in the "radical humanist" and "radical structuralist" dimensions of Burrell and Morgan's framework (1979). These paradigms concentrate on the creation of change; they focus on the oppositions, conflicts, and contradictions in society, and seek to undertake an advocacy or emancipatory role (Myers, 1997). Creswell (2003) identifies similar elements in his definition of advocacy and/or participatory knowledge claims. In this definition, Creswell posits that research should incorporate reform that may result in changes to the everyday lives of participants.

\subsubsection{Pragmatist paradigm}

Pragmatists link the choice of approach directly to the purpose the nature of the research questions posed (Creswell, 2003). In this paradigm, researchers are less concerned with antecedent conditions, and rather focus on the resulting actions, situations, and consequences of an inquiry (Creswell, 2003). The pragmatic approach is often adopted in mixed method studies where the use of pluralistic approaches is seen as advantageous to the research outcomes and thus, researchers may adopt both qualitative and quantitative approaches to research (Creswell, 2003). Tashakkori and Teddlie (1998) posit that the pragmatic paradigm both supports the use of mixed methods research and is applicable to both social and behavioural research. In essence, the pragmatist's view of the world is such that while they accept the positivist and post-positivist belief that there is an external reality (Creswell, 2003; Crotty, 1998), they contest the existence of an absolute truth (Tashakkori \& Teddlie, 1998). 


\subsubsection{Justification of the philosophical stance adopted in this study}

The adoption of a post-positivist philosophical stance for this research has been guided by two considerations; the intended goals of the research and the worldview of the researcher.

\section{Intended goals of the research}

The purpose of this research is to develop a better understanding of the phenomenon of knowledge sharing as it applies to the context of the public sector. Research has revealed that a range of perspectives and factors influence the practice of knowledge sharing. Thus, in keeping with the post-positivist stance of commencing a study with some element of established theory (Creswell, 2003), the researcher has been able to identify a broad range of a priori factors that are applicable to studies of knowledge sharing, and that form the basis of this exploratory study. The adoption of a postpositivist stance is also supported through the exploration and analysis of the study findings which will enable the researcher to deduce what role, if any, these a priori factors play within the specific context of the study (Creswell, 2003).

\section{The researcher's worldview}

The second factor that must be considered in adopting a philosophical stance is the researcher's own world view and recognising the assumptions that enable the researcher to make sense of the world. Eagleton (2003, in Ryan 2006) asserts that the postpositivist worldview, though still objective and believing in a single reality, considers not just the factors, but also recognises the importance of context in deriving meaning and understanding of a situation. Thus, context is an important factor in making sense of the world (Crotty, 1998). Ryan (2006) argues that post-positivists "learn from", rather than "test" reality. This fits comfortably with the both the aims of this research and the way in which this researcher acts within the world.

Accordingly, it is the combination of the a priori factors, and the context within which they are studied that will form the basis of the study findings. In addition, the patterns observed in this research will be reflected upon to draw inferences that can be generalised from the sample to a stated population (Orlikowski \& Baroudi, 1991). 


\subsection{Research methodology}

All research is guided by a defined research methodology. The methodology prescribes the methods by which research data are gathered and analysed. The overarching research strategy adopted in this study is case research. This section provides an overview of case research, and describes the reasons for the selection of multiple case research as the research strategy employed within this study.

\subsubsection{Case study research}

A case study is an empirical enquiry that investigates a contemporary phenomenon within its real-life context, especially where the boundaries between phenomenon and context are not clearly defined (Yin, 1989). Creswell (2003, p. 15) defines case study as one where the research "explores an event, activity, process or one or more individuals in depth".

Case research has been identified as a particularly suitable research method for exploratory studies (Trauth, 1987), for investigating phenomena within their natural settings (Benbasat, Goldstein \& Meads, 1987). Case research has been widely used for exploration and hypothesis generation (Benbasat et al., 1987). Table 13 presents a comprehensive synopsis of the key characteristics of case research as identified by Benbasat et al. (1987).

Case research enables rich data to be collected, and provides an "intensive, holistic description and analysis" (Merriam, 1998, p. 193). Although Yin (1993) opines that case study can benefit from the prior development of theoretical propositions, development of these theoretical propositions may be more difficult when there is little a priori knowledge. Indeed, it is this lack of a priori knowledge that is proposed as justification for a case study (Benbasat et al., 1987). Case research is particularly appropriate for IS research where the focus has moved from a study of technical issues to one that one that regularly focuses on issues of an organisational nature (Benbasat et al., 1987). Case research enables IS researchers to understand the interactions that arise from the complex interplay between people, organisations and technology (Dube \& Pare, 2003). 
Table 13: Key characteristics of case research (Benbasat et al., 1987)

\begin{tabular}{|l|}
\hline Key Characteristics of Case Research \\
\hline Phenomenon is examined in a natural setting \\
\hline Data are collected by multiple means \\
\hline One or few entities (person, group, or organisation) are examined \\
\hline The complexity of the unit is studied intensively. \\
\hline $\begin{array}{l}\text { Case studies are more suitable for the exploration, classification and hypothesis development stages of } \\
\text { the knowledge building process; the investigator should have a receptive attitude towards exploration }\end{array}$ \\
\hline The investigator may not specify the set of independent and dependent variables in advance. \\
\hline The results derived depend heavily on the integrative powers of the investigator \\
\hline $\begin{array}{l}\text { Changes in site selection and data collection methods could take place as the investigator develops new } \\
\text { hypotheses }\end{array}$ \\
\hline $\begin{array}{l}\text { Case research is useful in the study of "why" and "how" questions because these deal with operational } \\
\text { links to be traced over time rather than with frequency or incidence }\end{array}$ \\
\hline The focus is on contemporary events
\end{tabular}

The choice of philosophical stance through which a case study is conducted is dependent on the epistemological and ontological stances adopted within the study. While case studies most commonly use qualitative research methods, quantitative methods may also be incorporated (Merriam, 1998). Creswell (2003) posits that a mixed methods approach, incorporating both qualitative and quantitative methods, can help overcome weaknesses of a single methodological approach (see Section 3.3.1). There are several methods that may be used to gather data in case research, including documentation, interviews, direct observation, archival records, participant observation and physical artefacts (Yin, 2003).

Case study research can involve either single or multiple cases. Single case study is appropriate for studies where the case is unique; or where the research problem is of an exploratory nature (Yin, 1989). This view is supported by Remenyi, Williams, Money, \& Swartz (1998) who state that a case study allows the researcher to obtain a more holistic perspective because the focus is on learning about the organisation's process. Merriam (1998) states that policy and practice can be directly influenced by case study research because of the rich and holistic insight it provides into the phenomenon. However, single case study research has been criticised as an inadequate methodology in terms of inferring generalisability from the sample to a general population (Lee, 1989; Tellis, 1997). To mitigate the limitations of case research, researchers can extend the research to incorporate multiple case studies.

\subsubsection{Multiple case research}

Conducting multiple case research better supports generalising the research findings and helps to strengthen the precision, validity, and stability of the research (Benbasat et al., 
1987; Miles \& Huberman, 1994; Yin, 2003). These benefits can outweigh the additional time and resource considerations that conducting multiple case research can generate. Benbasat et al. (1987, p. 373) advise that multiple case design "is desirable when the intent of the research is description, theory building, or theory testing."

Multiple case studies enable the comparison of evidence, better data triangulation (Yin, 1989), and the use of procedures for coding and analysis (Lincoln \& Guba, 1985; Miles \& Huberman, 1994). The richness of the data collection also allows the development of a strong base for cross-case analysis (Lincoln \& Guba, 1985; Miles \& Huberman, 1994). Stake (1995) concurs and argues that multiple case research can produce valid modification and naturalistic generalisations.

Selection of case study sites is a key consideration in multiple case research. Yin (1994) advises researchers to select either sites where similar results can be predicted, thereby adopting a literal replication strategy, or sites where contradictory results are predicted thus facilitating theoretical replication. Ensuring the cases represent an appropriate population helps to define the research limits and is effective in assisting the researcher to control irrelevant variations (Eisenhardt, 1987).

\subsubsection{Justification of case research methodology}

Several factors have contributed to the selection of multiple case research as the methodology of choice for this study.

Firstly, the exploratory nature of the study fits within the recommended uses of case research (Benbasat et al., 1987). While research into the phenomenon of knowledge sharing continues to grow, there has been very little research that investigates the phenomenon in the context of the public sector. This research aims to provide scholars with a better understanding of knowledge sharing within the public sector. The research questions are concerned with how and why knowledge is shared, and are therefore exploratory in nature.

Secondly, the context of the research is an important aspect of the research study. The extant literature questions whether knowledge sharing approaches developed in the private sector are appropriate or suitable for use in the public sector. Therefore study of 
the phenomenon within its natural setting is required in order to better understand the phenomena in this context.

Thirdly, Yin (2003) posits that exploration of a contemporary phenomenon can be effectively addressed by case research methodology. In this study, the interorganisational context and focus on SC both represent examples of contemporary phenomenon that have not been fully explored.

Finally, Dube \& Pare (2003) identify multiple case research as particularly suited to research where there is little established theory. Though the conceptual framework has been established to guide the study and identifies a priori factors from the extant knowledge sharing literature, there is little established theory relating to interorganisational knowledge sharing in the context of the public sector.

The researcher's decision to conduct multiple case research was made to specifically address the lack of generalisability arising from single case research. The findings derived from multiple case research enable the comparison of evidence across cases, support better data triangulation, and address issues related to the validity of qualitative research.

\subsection{Mixed method research}

This section discusses the concept of mixed methods design and the justification for its use in this research. The section concludes by presenting an overview of SNA as a research method embedded within the case research methodology.

\subsubsection{An overview of mixed methods design}

The first step toward credible findings from a research study lies in the careful planning of the study itself, and can reduce or limit the criticisms stemming from lack of rigour (Yin, 1993). A mixed method design includes quantitative and qualitative data in the same study (Creswell, 2003). Quantitative data is data in numerical form, often derived from surveys or structured interviews. Taylor et al. (1995) describe qualitative data as "descriptive data from participant observation and unstructured interviews to information from written sources, such as diaries, autobiographies and novels" (p. 632). Collecting data through a combination of methods provides a fuller picture of the 
phenomenon (Bonoma, 1985). Case research supports multiple data collection methods, including interviewing, document analysis, participant observation, and use of surveys (Miles \& Huberman, 1994; Yin, 1994). In this study, quantitative data is gathered via survey and the use of SNA as a specific research method (see Section 3.3.4). Qualitative data is gathered through unstructured interviews and open-ended questions incorporated into the survey.

The advantages of conducting mixed methods research enable the phenomena to be studied through the use of multiple data collection techniques thus enabling the generation of multiple data sets (Sawyer, 2001). While the findings of single method research may be challenged, findings that are have been developed through a variety of methods may be more difficult to contest (Petter \& Gallivan, 2004). Thus, the use of a mixed methods approach can provide a more complete picture of a research problem (Creswell, 2003), can help to address issues of validity and rigour, and provide triangulation of data - often identified as weaknesses of the use of a single methodology.

Opponents to the mixing of quantitative and qualitative methods question the appropriateness of combining methods that have been developed under different paradigms, where each paradigm is based on different assumptions. Mingers \& Brocklesby (1997), however, argue that research methods are able to be separated from the paradigm from which they emerged.

The adoption of a mixed methods design can also impact on the time and expertise required of the researcher as it requires both qualitative and quantitative skills, and the collection and analysis of data by several methods is likely to require more time than data collected through single method.

Creswell (2003) proposes four decisions that must be taken in order to determine the mixed methods strategy of enquiry. Firstly, the research must decide on the implementation sequence of the data collection. The three main approaches to mixed methods research include the sequential, concurrent, and transformative approaches (Creswell, 2003). The sequential approach involves the use of qualitative and quantitative methods one after another. The concurrent approach uses both methods at the same time. The transformative approach adopts a theoretical lens "as an overarching perspective within a design that contains both quantitative and qualitative 
data" (Creswell, 2003, p. 16). The second decision concerns the priority that will be given to the quantitative and qualitative data collection and analysis. Thirdly, it must be decided at what stage the data and findings will be integrated. Integration can occur at collection, analysis, and integration stages. The final decision relates to whether the research will adopt an overall theoretical perspective that will be used in the study.

\subsubsection{The mixed methods design of this study}

\section{Implementation sequence}

This study employs a sequential exploratory design as shown in Figure 18. This design is appropriate for studies of an exploratory nature (Creswell, 2003), and will enable the researcher to expand the findings derived from each phase of the research.

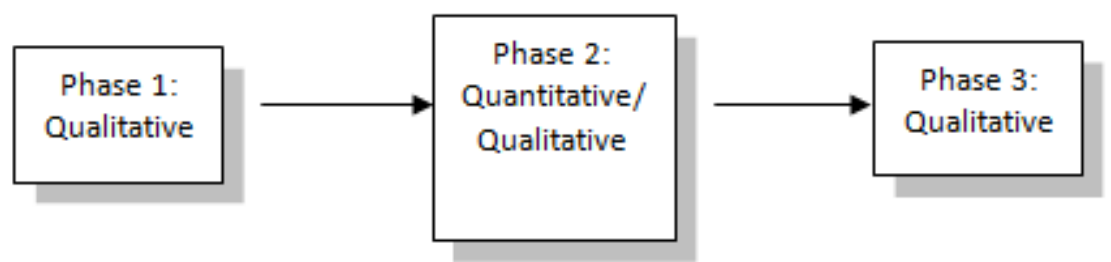

Figure 18: Overview of the research phases

Creswell (2003, p. 16) confirms the sequential approach as appropriate for a study in which the researcher "seeks to elaborate on or expand the findings of one method with another method". Phase 1 of the study provides the researcher with an opportunity to gather contextual data about each of the cases, to develop a high level overview of each case, and the opportunity to fine-tune the survey instrument that has been developed to gather data in Phase 2 of the study. The second phase of data collection seeks to generalise the data gathered in Phase 1 to the wider population. The Phase 2 survey gathers qualitative data through the use of open questions, as well as quantitative data through the collection of social network data based on the patterns of interaction in each case, and nominal data relating to the main themes of the study. The final phase of data collection will enable the researcher to discuss and confirm the data gathered in the initial phases of data gathering and to explore factors in greater depth. The specific research methods included semi-structured interviews and a survey incorporating open, closed, and social network related questions (see Table 14). 
Table 14: The research methods

\begin{tabular}{|l|l|l|}
\hline Phase & Type & Method \\
\hline One & Qualitative & Semi-structured interviews \\
\hline Two & Quantitative & $\begin{array}{l}\text { Social Network Analysis } \\
\text { Survey: closed questions }\end{array}$ \\
& Qualitative & Survey: open questions \\
\hline Three & Qualitative & Semi-structured interviews \\
\hline
\end{tabular}

\section{Prioritisation of data types}

The second research design decision relates to the priority or weighting that is applied to the qualitative and quantitative data. As shown in Figure 18, qualitative data is the predominant data gathered in the study. Qualitative data are gathered in each of the discrete phases of the research through the use of semi-structured interviews (Phases 1 and 3), and open ended survey questions in Phase 2. Quantitative data are gathered only during Phase 2 of the study and consists of SNA data and nominal data collected through closed questions. Accordingly, the majority of data collected in this study is categorised as qualitative data and greater priority is given to this aspect.

\section{Integration of data and findings}

Creswell's third decision point relates to the integration of data. Integration can occur at one or several points throughout a study during the collection, analysis, and interpretation stages. In this study, data are integrated at collection through use of a survey incorporating both quantitative and qualitative data. During analysis, the qualitative data from both Phases 1 and 3 are integrated through the use of NVIVO, and used to add context and meaning to the quantitative data. Though the social network data is not formally integrated with the qualitative data, direct comparisons are made between the two.

\section{Overarching philosophical perspective}

The final decision proposed by Creswell (2003) concerns the use of research methods that have been developed under different philosophical paradigms. A number of philosophical perspectives have been detailed in Section 3.1, and the researcher has adopted post-positivism as the overarching philosophical stance of the study (see Section 3.1.5). 


\subsubsection{Addressing qualitative data validity issues}

The predominant method of data collection and analysis focuses on qualitative data. This data is collected via semi-structured interviews in Phases One and Three, and through open-ended survey questions in Phase 2.

Issues of validity in qualitative research differ to those in the quantitative paradigm. This study adopts Guba and Lincoln's (1994) guidelines to establish the "soundness" of qualitative research. These guidelines offer an alternative to those undertaken in quantitative research and offer a more relevant approach to the epistemological perspective adopted by qualitative researchers. These alternatives are presented in Table 15 .

Table 15 : Criteria for assessing qualitative and quantitative research (Guba \& Lincoln, 1994)

\begin{tabular}{|l|l|}
\hline Qualitative Research & Quantitative Research \\
\hline Credibility & Internal Validity \\
\hline Transferability & External Validity \\
\hline Dependability & Reliability \\
\hline Confirmability & Objectivity \\
\hline
\end{tabular}

Scholars have argued that case study research presents limited scope for generalisability. Maxwell (1992) contends that there is a clear distinction between the degree to which qualitative and quantitative studies can be generalised to other contexts and settings. Several techniques which can be used to establish generalisability are employed in this study. As noted in Section 3.2.2, multiple case studies enable the comparison of evidence, better data triangulation (Yin, 1989), and the use of procedures for coding and analysis (Lincoln \& Guba, 1985; Miles \& Huberman, 1994). The richness of the data collection also allows the development of a strong base for crosscase analysis (Lincoln \& Guba, 1985; Miles \& Huberman, 1994). Stake (1995) concurs and argues that multiple case research can produce valid modification and naturalistic generalisations.

Multiple cases also enhance the credibility of the research (Lincoln \& Guba, 1995). Credibility in qualitative research, looks to the establishment of the case phenomenon in a reliable and believable manner (Miles \& Huberman, 1994; Yin, 1993), through the composition of a chain of evidence flowing from the research questions through to the final outcome of the study (Yin, 1993). As undertaken in this study, the prior development of an interview guide assists credibility (Yin, 1993) as well as careful 
selection of interviewees and structured processes for both interviewing and recording and transcribing of the interview (Lincoln \& Guba, 1995). All of these measures have been adopted in this study.

Dependability is defined as the extent to which the study, if replicated "with the same or similar respondents (subjects) in the same (or similar) context, its findings would be repeated" (Erlandson, Harris, Skipper, \& Allen, 1993, p. 33). To maintain dependability, the researcher must maintain a record or audit trail that details how the research has been carried out. This study accommodates dependability through the use of a research journal to record actions and decisions relating to the research, by setting out a clear research process, detailing aspects of the research such as the interview protocol and survey, and the creation of full interview transcripts.

Confirmability refers to the degree to which the research results can be confirmed or substantiated by others. Strategies to enhance confirmability include implementing a data audit or locating research cases that contradict prior observations. In this study, a data audit was initiated from the outset. This audit includes the raw data, personal notes and observations, memos, and clearly documented data collection and analysis procedures.

\subsubsection{Social network analysis}

The primary research strategy for this study is case research. SNA is an embedded research method within the overall case research. This section introduces SNA and outlines the key concepts including the ways in which data may be collected and analysed. The section concludes by discussing validity and ethical issues that should be considered when undertaking SNA

\section{An overview of social network analysis}

SNA is the study of social relations among a set of individuals (actors) and seeks to understand the actors' beliefs and behaviours as a function of the structure of relationships in which they occur. A social network consists of a finite set or sets of actors and the pattern of relational ties that exist among them (Wasserman \& Faust, 1994). Applications of SNA are wide-ranging and include organisational behaviour and development, epidemiology, terrorist networks, and KM. SNA is most commonly used 
as a quantitative method for mapping and visualising social network relations, however qualitative research on social networks has also been conducted, exploring topics ranging from community studies to SC (Edwards, 2010). In this research, the adoption of mixed methods research combines the quantitative SNA data with qualitative data that provides context and deeper meaning to the research findings.

SNA has also been used extensively in knowledge related studies and has been used in research streams including SC, knowledge embeddedness, knowledge transfer, network organisations, and KM (Borgatti \& Foster, 2003; Kilduff \& Tsai, 2003; Su, Huang \& Contractor, 2010). Within the sphere of KM, SNA is most often used to map and understand the flow of knowledge along pathways in an organisation. SNA allows for pivotal team members to be quickly identified, as well as determining which actors may be more isolated within the network. A network approach differs to that of a more traditional, non-network approach. In a non-network research approach, actors are assigned to a common group according to their titles, occupations, roles etcetera, after which interactions can be analysed (Haythornthwaite, 1996). By contrast, SNA allows the researcher to analyse the interactions in order to assign individuals to a group. Haythornthwaite (1996) asserts that this type of dichotomy underpins the concepts of formal and informal exchange of information within organisations.

SNA can be conducted at the individual, group, or organisational level and focuses on the patterns of relationships between actors as well as identifying available resources and their exchange (Scott, 1991). At an individual level of analysis, the focus is on an individual and the connections of that individual. At a group level, it is an excellent tool to describe the individual aspects of a project network (Mead, 2001), and is particularly useful for identifying and exploring important aspects of group interaction (Katz et al, 2004). At this level, it may also serve as the starting point for study of group structural properties (Pearce \& David, 1983). At an organisational level, SNA enables organisations to measure, analyse, and describe interaction patterns within the organisation (Cross \& Prusak, 2002). SNA is also relevant at the inter-organisational level whereby a clearer picture of direct and indirect inter-organisational relationships may be seen through the application of a network approach (Tichy, Tushman, \& Fombrun, 1979). 


\subsubsection{Fundamental concepts of social network analysis}

There are a number of fundamental concepts within SNA that are widely understood and which are essential to all studies.

\section{Unit of analysis}

Units of analysis within an SNA study are referred to as actors: an actor can be an individual, a group, an organisation, or some form of collective social unit. Depending on the number of actors involved in a relational tie, the tie will be denoted as a dyad, the tie between two actors; a triad, the ties between a subset of three actors; or a subgroup which refers to a larger number of actors than either a dyad or triad. A group is a finite collection of actors on which ties are to be measured (Wasserman \& Faust, 1994).

\section{Ties}

A relational tie links actors to each other. It is through these relational ties that actors may exchange resources such as information, goods and services, or financial support (Haythornthwaite, 1999). The relational ties that occur between actors may vary in content, direction, and strength (Garton, Haythornthwaite, \& Wellman, 1997). In terms of content, relationships can include the sharing, delivery, or exchange of a wide variety of resources, including information. Direction denotes the flow of information from one person to another. This flow can be uni-directional (one-way) or bidirectional (reciprocal). Tie strength is indicative of the intensity of the relationship (Haythornthwaite, 1996), and is often signified by the amount of exchange or the frequency of exchange between actors.

The concept of tie strength is a key aspect of SNA research. Haythornthwaite (1999) defines tie strength between actors by the extent to which the strength of a tie is dependent on the number and types of relationships which a pair maintains, and on the strength of each individual relationship. Granovetter (1973) points to the combination of time, emotional intensity, the level of mutual confidence and the reciprocal services that combine to represent the strength of a tie. Tie strength is important in the assessment of the overall connectedness of actors in an environment and the likelihood that information will flow from one actor to another. Tie strength varies along a continuum from weak to strong. Traditionally strong ties have been understood to be 
particularly helpful in the exchange of information as a strong tie denotes a more intimate relationship. There is also a higher likelihood that these ties will be reciprocal. Actors with strong ties are more likely to show similarities in attitudes, background, experiences, and access to resources (McPherson, Smith-Lovin, \& Cook, 2001). By contrast, weak ties denote relationships that are infrequent and distant, but often enable actors to access more diverse information (Hansen, 1999). Granovetter (1973) argues that in some instances, weak ties can be superior to strong ties as they require less investment in terms of time and emotional intensity.

\subsubsection{Quantitative social network analysis metrics}

SNA provides the researcher with a range of metrics that can be employed to measure and assess the characteristics of an entire network, a subgroup, or individual.

\section{Network level}

At a network level, typical measures include network size, density and distance, and network centralisation. These can be important aides in enabling the researcher to determine how well a network is connected, or how easily knowledge flows through the network (Durland, 2003). Network size simply denotes the total number of actors within the network. Hanneman \& Riddle (2005) identify size as critical for the structure of social relations based on an actor's ability to build and maintain ties. Measuring the density of a network describes the overall connectedness of the network and gives a general measure of the health of the network. Density indicates the overall connectedness of the network by measuring how many connections there are between actors (team members) compared to the maximum possible number of connections. Higher density measures indicate a higher level of connectedness across the network. Distance measures indicate how long it will take to access information if an actor is not directly connected to another. Network centralisation is concerned with the extent to which the network has a centralised structure and is therefore an indicator of the overall cohesion or integration of the network (Scott, 1991). While a general level of network cohesion is identified by the network's density measure, network centralisation describes the degree to which that cohesion is focused around particular actors. These network level measures are summarised in Table 16. 
Table 16: Quantitative SNA measures at the network level

\begin{tabular}{|l|l|l|}
\hline Measure & Level & Description \\
\hline Density & Network & $\begin{array}{l}\text { Measures the overall connectedness of the network by measuring how } \\
\text { many connections there are between actors (team members) compared } \\
\text { to the maximum possible number of connections. Higher density } \\
\text { measures indicated a higher level of connectedness across the network. }\end{array}$ \\
\hline Distance & Network & $\begin{array}{l}\text { Distance measures the shortest path to each actor to whom a direct path } \\
\text { does not exist. High distance measures indicate that information will take } \\
\text { longer to travel through the network. Low distance measures indicate } \\
\text { that information is more easily accessible. }\end{array}$ \\
\hline $\begin{array}{l}\text { Network } \\
\text { Centralisation }\end{array}$ & Network & $\begin{array}{l}\text { The most centralised network is a star shape, where all the people at the } \\
\text { edge of the network are directly tied to one person at the centre, but not } \\
\text { to each other. Network degree centralisation compares the observed } \\
\text { centralisation in a network (how focused it is around particular people) } \\
\text { with this perfect star-shaped scenario (i.e. with the maximum amount it } \\
\text { possibly could be) for a network of the same size and expresses this as a } \\
\text { percentage. }\end{array}$ \\
\hline
\end{tabular}

Subgroup level

Pearce \& David (1983) suggest that SNA may also serve as the starting point for study of group structural properties as shown in Table 17. These measures can be used to define how communication flows in organisations (Pearce \& David, 1983).

Table 17: Research of group structural properties (adapted from Pearce \& David, 1983)

\begin{tabular}{|c|c|c|}
\hline Structural Property & Description & Original Source \\
\hline Connectedness & $\begin{array}{l}\text { Extent to which groups members } \\
\text { identify with the goals of other } \\
\text { members of their groups; a measure } \\
\text { of group cohesiveness }\end{array}$ & O'Reilly \& Roberts, 1977 \\
\hline Reciprocity & $\begin{array}{l}\text { Degree to which there is two way } \\
\text { communication in a workgroup }\end{array}$ & Newcomb, 1979 \\
\hline Vertical Differentiation & $\begin{array}{l}\text { Degree to which different } \\
\text { organisational hierarchylevels are } \\
\text { represented in a given work group } \\
\text { network }\end{array}$ & O'Reilly \& Roberts, 1977 \\
\hline $\begin{array}{l}\text { Horizontal } \\
\text { differentiation }\end{array}$ & $\begin{array}{l}\text { Degree to which different job areas } \\
\text { are represented in a given work group }\end{array}$ & Mohr, 1979 \\
\hline Coalitions & $\begin{array}{l}\text { Perceived linkages among several } \\
\text { individuals who believe that their } \\
\text { ability to dominate organisational } \\
\text { relationship is greater as a group than } \\
\text { an individuals }\end{array}$ & Thibaut \& Kelley, 1959 \\
\hline
\end{tabular}


Individual level

At an individual level, SNA can be used to define the roles and position of individual actors within the network (Cross \& Prusak, 2002). By employing a range of centrality measures, SNA can ascertain how well an individual is embedded within a network, as well as the level of power or influence they are likely to hold within the network.

Degree centrality defines how well connected an actor is within the network (Scott, 1991) and is calculated by counting the number of other actors to whom they are connected regardless of whether that tie is one-way or reciprocal. Degree centrality can be further broken down through the calculation of in-degree and out-degree measures. In-degree measures calculate the number of incoming connections the actor receives. For example, in an advice seeking network, an actor who receives requests for advice from five other actors would be represented by an in-degree score of 5. An out-degree score refers to the number of request the actor makes of other actors. So, if an actor seeks advice from three others, then their out-degree score is 3.

Betweenness centrality measures the degree to which an actor lies between other actors in the network (Scott, 1991). This measure indicates an actor's position within a network and relates to their ability to control information by acting as an intermediary between other actors (Wasserman \& Faust, 1994). Betweenness is often described as a measure of gate-keeping, and is therefore considered to denote a position of strategic advantage and information control (Hawe \& Ghali, 2007).

Closeness centrality measures the degree to which an actor is near all other actors in a network (Hanneman \& Riddle, 2005). Actors with high closeness centrality are able to reach lots of other actors within the network (Kilduff \& Tsai, 2003).

Individual level SNA measures are summarised in Table $\mathbf{1 8 .}$ 
Table 18: Quantitative SNA measures at the individual level

\begin{tabular}{|l|l|l|}
\hline Measure & Level & Description \\
\hline Degree Centrality & Actor & $\begin{array}{l}\text { Measures the number of direct connections an actor has. Connections } \\
\text { are broken down into: } \\
\text { In-degrees: The total number of incoming connections (i.e. those } \\
\text { seeking information for this actor) } \\
\text { Out-degrees: The total number of outgoing connections (i.e. the } \\
\text { number of information seeking requests this actor makes of others) }\end{array}$ \\
\hline Betweenness & Actor & $\begin{array}{l}\text { The degree to which an actor lies between other individuals in the } \\
\text { network, and may therefore act as a broker or bridge between actors }\end{array}$ \\
\hline Closentrality & & The degree to which an actor is near all other actors in a network. \\
\hline
\end{tabular}

\section{SNA sociograms}

SNA findings are depicted in sociograms as shown in Figure 19. Each circle (node) represents a team member who was nominated by another team member as someone they would go to for work-related help or advice. The colour of the node may depict a characteristic of that person, such as their gender, role, or the team they belong to. The direction of the arrows indicates the flow of information from that node. For example, in the network below, red nodes represent males, and blue nodes represent females. Joe seeks information from Kevin, Kevin seeks information from Jill, and Jill seeks information from Kevin. Therefore Kevin and Jill have a reciprocal information seeking relationship. Sandra has no relationships with Jill, Kevin, or Joe and is therefore isolated from the network. Joe can also be considered a peripheral player in the network as no-one seeks information from him.

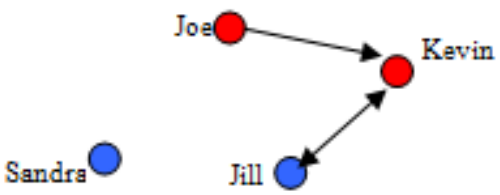

Figure 19: SNA sociogram

\subsubsection{Data collection techniques}

Depending on the purpose of the research, there are several ways in which data may be collected including questionnaires, interviews, observation, experiments, and archival records (Wasserman \& Faust, 1994). Of these, the questionnaire is most commonly used to collect data in network analysis studies and will be the instrument of choice in this study. Wasserman \& Faust (1994) identify three different question formats: 
Roster versus Free Recall: The roster format can be used when all the members of a set are known to the researcher prior to commencement of a study. These members are included on the questionnaire given to each participant. By contrast, a free recall format does not list members of a set, but requests each participant to identify actors with whom they have ties.

Free versus Fixed Choice: Free format places no limit on the number of people the participants can identify. By contrast, fixed choice formats require participants to name a specific number of other actors.

Ratings versus Complete Ranking: As the title indicates, complete ranking requires participants to rank a particular thing from top to bottom, least to most, etcetera, whereas a rating requires that they rate a particular item/person/relationship etc.

\subsubsection{Issues for consideration in social network analysis use}

Borgatti \& Molina (2003) raise some interesting issues that must be considered when undertaking network analysis. Unlike the majority of conventional studies, the focus of network studies is to map relationships among research participants - therefore it is vital that participants be identified. Thus, anonymity of research participants at the data collection stage is not possible (Borgatti \& Molina, 2003). The researcher must therefore consider how this is likely to influence the data gathered during the study. One obvious solution, as suggested by Borgatti \& Molina, is to ensure confidentiality through the use of disguised names or untraceable identification numbers in regard to all analyses and reports generated from the data.

Difficulties may also arise if any organisational members opt out of the study, leading to important gaps in the resulting network analysis. Further, active study participants may still refer to these participants. This raises complex issues for the researcher in terms of their inclusion or non-inclusion. Should the researcher opt to exclude all references to the non-consenting participant, the analysis may be considered incomplete and potentially flawed. Although Borgatti \& Molina (2003) argue that an individual's perceptions of their fellows and their relationships with them are essentially their own opinions, they caution that consideration should be given to overcoming this difficulty during the ethical consent process. 
Validity, reliability, accuracy or error

With all research, it is important to examine the validity, reliability, and accuracy or error of a study. These concepts are of particular importance in the domain of SNA as, in many cases, data is gathered through actors' self-reporting. Wasserman \& Faust (1994) point out that there appears to have been little research carried out on these issues.

There are two major areas of potential inaccuracy in SNA. Bernard, Killworth, and Sailor's (1980, cited in Wasserman \& Faust, 1994) concluded that when reporting on their own interactions, about half of what is reported is incorrect in some way; secondly, when the actor is an organisation, rather than an individual, it is important to ascertain that the provider of information on behalf of the organisation, is able to provide accurate information.

Similarly, there is little research on the construct validity of measures of network concepts, the extent to which it actually measures what it is intended to measure. A variable or concept is presumed to be reliable when the same results are achieved through repeated measurements. Wasserman \& Faust (1994) report that questions using ratings or full rank orders are more reliable than fixed choice designs.

The difference between the true and observed values is denoted by measurement error. Wasserman \& Faust (1994) point to the error that can arise where data is collected in a fixed choice format. The error may occur because participants are required to list a particular number of responses, although they may have less or more "true" answers to the questions.

In this study, the issue of potential social network data inaccuracy has been addressed through the adoption of a mixed methods study design. This enabled the researcher to collect data through multiple methods and thus increase accuracy through triangulation of the data. Further, the survey design incorporated the use of ratings as recommended by Wasserman \& Faust (1994). 


\subsubsection{Unit of analysis}

Selecting the unit of analysis for case research should involve consideration of the purpose of the research and the associated research questions, as well as the types of generalisations the study aims to develop (Benbasat et al., 1987). The context in which the phenomenon is studied should also be considered (Yin, 2003).

This research explores the phenomenon of inter-organisational knowledge sharing in the public sector. Each case consists of a number of individuals who represent a number of individual public sector organisations. The main focus of the researcher is to better understand how knowledge is shared within each of the cases. The study will contrast the formal team structure with the informal structure revealed in the network analysis, and will explore the role that ICT and SC play within each of the cases. The use of multiple cases will enable the findings to be cross-analysed. Therefore the unit of analysis in this study is the team. However, it is anticipated that the study will also generate findings and implications at the individual and inter-organisational level.

\subsection{Research design}

This section presents the research design used in this study. The section explains how case study sites were selected, and describes the methods and tools used to collect and analyse data within each phase of the study.

\subsubsection{Selection of the case study sites}

This study is based on the use of multiple cases. There are several aspects that must be considered when making decisions about which case sites which will be most suitable to the study.

\section{Establishing the selection criteria}

Yin (1984) advises that the selection of cases in multiple case research should focus on either sites where similar results can be predicted, thereby adopting a literal replication strategy; or sites where contradictory results are predicted thus facilitating theoretical replication. 
The broad criteria for case study selection were:

1. Organisations operating within the New Zealand public sector, including Public Service Departments; Non-Public Service Departments; Crown Entities; Public Finance Act Fourth Schedule Organisations; the Reserve Bank of New Zealand; Offices of Parliament; and State Owned Enterprises.

2. Each organisation must be involved with one or more public sector organisations in the context of an inter-organisational collaboration venture.

3. Each participating organisation must have one or more staff members participating in the inter-organisational collaborative venture.

4. All organisations involved in the collaboration must consent to participate.

5. Individual members of the inter-organisational teams must consent to participate.

These criteria allowed for the identification of sites of a similar nature and that would most likely produce similar results, as required in literal replication strategy (Yin, 1994). In addition, the nature of the SNA necessitated the participation of all team members - otherwise incomplete network data would be gathered. This added a significant level of complexity to the identification and selection of potential case study sites.

Yin (1994) states that the number of cases selected for a research purpose depends on the certainty of the results the researcher wants to achieve. However, practical considerations often form part of the decision regarding the number of cases (Pare \& Elam, 1997). In this study, time and resource restrictions together with the desire to adopt a literal replication strategy resulted in the selection of four case study sites.

\section{Identification of specific case sites}

The researcher commenced the search for case study sites in November 2005. To identify specific case sites the researcher analysed the organisations listed on the New Zealand Government's State Services Commission website to make a shortlist, based on 
the researcher's knowledge of public sector organisations ${ }^{4}$, of those most likely to be engaged in inter-organisational collaboration. An introductory letter was sent to the Chief Executives of each of the 44 identified organisations seeking expressions of interest in participating in the research (see Appendix 1). The researcher followed up each letter with a telephone call one week later. In most cases, a message was left and a note made to call again after one week if no response was received. This process took longer than anticipated, in part due to the Christmas period and also due to the fact that a general election had recently been held following which, due to New Zealand's system of MMP (Mixed Member Proportional), it had taken some time for the government to be determined. Twenty-two organisations did not respond to either the letter or the telephone contact. In twelve instances, letters of response were received declining to participate in the research. Four letters were receiving indicating interest in participation, and a further six organisations indicated interest through telephone conversations with the researcher.

By the end of February 2006, the researcher had identified six organisations that had indicated they were involved in an inter-organisational collaborative endeavour which would be suitable for the purposes of the study. In each case, a single individual who had responsibility for the collaborative team was identified. A preliminary email was sent to each of these individuals and this was followed up with a face-to-face meeting with each to discuss the research purpose and requirements in more detail. As a result of these meetings, one of the cases was identified as unsuitable for the research due to the specific nature of the case, and another withdrew. Four organisations confirmed they would be happy to proceed further.

At this stage, the securing of cases became particularly complex. Although individual organisations had indicated an interest in participating, in order to qualify as a case study, and to be able to collect meaningful data, all organisations participating in each collaboration needed to consent to participate. Initial contact with members of other participating organisations was made through the consenting organisation. In one instance, the researcher was required to meet with one of the organisations to supply

\footnotetext{
${ }^{4}$ The researcher has been employed in a variety of roles within public sector organisations and has also provided consultancy services to the sector through a private consultancy firm.
} 
further detail and answer the organisation's specific questions. By April 2006, all organisations had agreed their participation and had supplied the researcher with contact details for each member of each organisation participating in the research. In total a list of 61 contacts was received. Each participant was sent an introductory email containing an outline of the study and details of how they could be involved (see Appendix 2). Each of these participants was able to decide for themselves if they wished to be part of the research. In total, five of the originally identified participants did not take part in the research. Of these, one declined to participate, two were on parental leave during the course of the research, one left the team prior to the commencement of data collection, and one did not respond. This affected three of the cases. In one case, discussion with the team leader identified the non-participant as somewhat removed from the day-to-day operations of the collaboration and consequently were rarely involved in day-today interactions within the team. Thus, omission of this individual was unlikely to reflect in the study results. In the case of the participants who were on parental leave, a decision was taken to move forward without these individuals. This decision had impact on the collection of social network data and this is discussed in the data collection section (see Section 3.4.2).

\subsubsection{The value of a pilot study}

Several scholars recommend conducting a pilot case prior to embarking on the full research study (Stake, 1995; Yin, 1994). A pilot study allows the testing of research instruments and identifies areas for potential refinement (Yin, 1994, Benbasat et al., 1987). In this research, Case 1 was identified as the pilot case study and data collection was commenced at the end of April 2006. Phases 1 and 2 of the pilot case were completed, and the analysis of the Phase 2 data identified several minor changes that were made to the Phase 2 survey instrument. The final three cases were confirmed during the collection of Phase 2 data from the pilot case. Consequently Phase 3 of the pilot case was completed concurrently with collection of Phase 1 data from the three remaining cases (see Figure 20). 


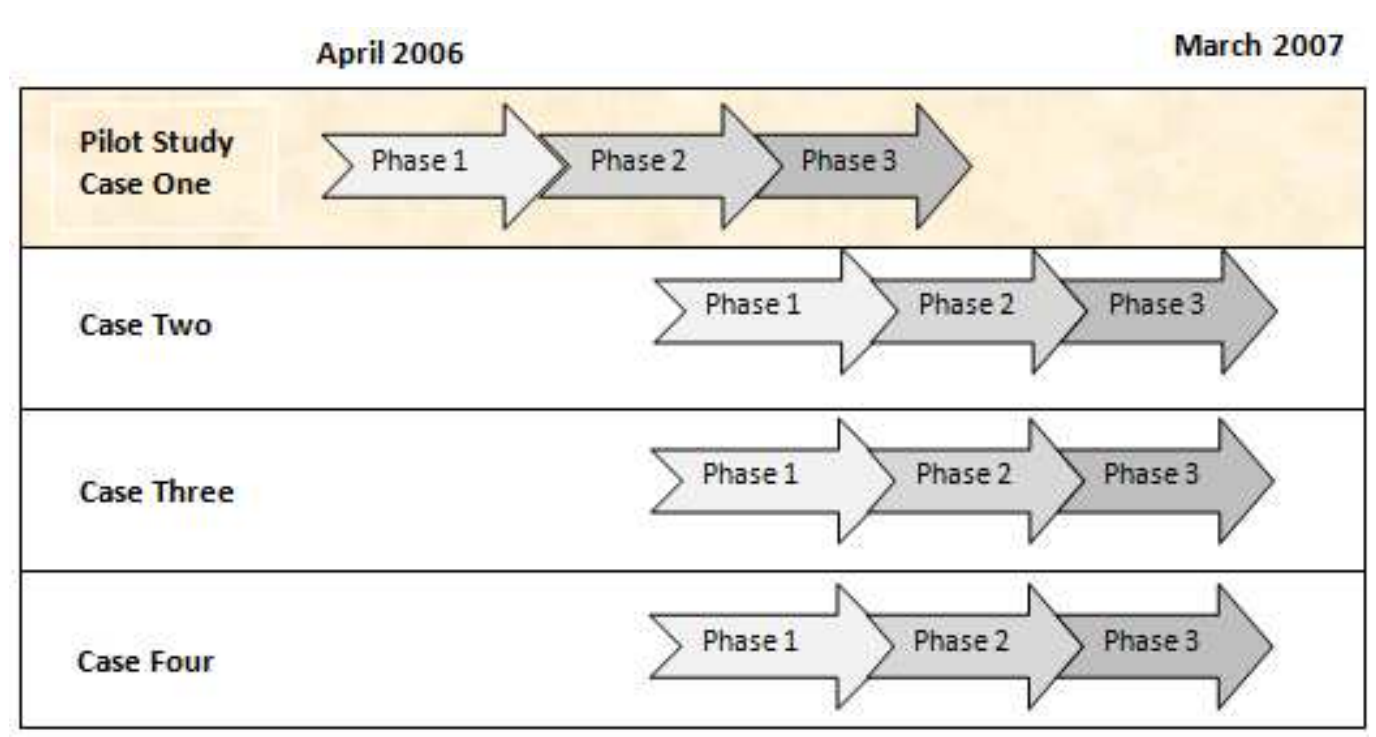

Figure 20: Research data collection \& analysis timeline

This meant that although the pilot case was not able to be completed in entirety before commencing data collection with the other cases, the Phase 2 survey instrument had been tested prior to its use in the other cases. The lack of opportunity to complete Phase 3 of the pilot study did not affect the design of the semi-structured interviews used in Phase 3 of the other cases. Phase 3 questions were based on the Phase 2 findings of each case, and as such were likely to differ slightly depending on the individual case findings.

\subsubsection{Data collection and analysis}

Due to the multi-phased, multi-method approach of this study, the researcher was cognisant that initial organisation of the data would be an important aspect in assisting the analysis process. Yin (1994) recommends the use of a database or repository for the storage of data as this will help to increase reliability of the findings. In multiple case studies, ensuring proper administration of case data is of particular importance because the details of individual sites may run together as time elapses (Benbasat et al., 1987). The nature of the data collected in this study necessitated the use of two data repositories. Qualitative data was stored and analysed using NVIVO (version 8), and the social network data collected from the survey was stored and analysed using UCINET. These tools are explained further in the following sections. 


\subsubsection{Phase 1: Qualitative data collection and analysis}

This section describes the collection and analysis of data collected during Phase 1 of the research.

\section{Data collection}

The purpose of Phase 1 data collection was to identify general data relating to the general conduct of inter-organisational collaboration as well as data relating to the specific case study. The interviews provided information regarding the structure of the team, and the use of any mandated processes or procedures and the locus of decision making. This data was also used in development of the Phase 2 survey. The design of the survey was consistent across all four cases.

Phase 1 qualitative data were collected through face-to-face, semi-structured interviews conducted with a selected number of participants across each of the four case studies as shown in Table 19.

Table 19: Phase 1 Interviews
\begin{tabular}{|l|r|l|l|}
\hline & $\begin{array}{l}\text { Total No of } \\
\text { Organisations }\end{array}$ & $\begin{array}{l}\text { Total Number of } \\
\text { Participants }\end{array}$ & $\begin{array}{l}\text { Interviews carried } \\
\text { out in Phase 1 }\end{array}$ \\
\hline Case 1 & 2 & 12 & 2 \\
\hline Case 2 & 5 & 10 & 2 \\
\hline Case 3 & 7 & 14 & 3 \\
\hline Case 4 & 8 & 20 & 2 \\
\hline & 22 & 56 & 9 \\
\hline
\end{tabular}

Interviews can be carried out with individuals or groups and can be conducted either face-to-face or by telephone or other communication means (Creswell, 2003). Face-toface semi-structured interviews are the most common case data collection method (Pare \& Elam, 1997), and enable the research to elicit the views and experiences of participants in their own words (Kaplan \& Maxwell, 1994). Creswell (2003) notes one of the advantages of interviews is that they allow the researcher control over the questioning.

These initial interviews always included the leader/s of the collaboration and, where possible, at least one other key staff member. The semi-structured nature of the interviews allowed for the prior identification of factors of interest to be explored in the 
study, but also allowed for unanticipated issues to emerge during the course of the interview process.

\section{Before the interview}

Each interview participant was contacted by email and invited to participate in the interview process. The email included an outline of the research; a consent form approved by the University's Human Ethics Committee; and, an interview protocol. (see Appendix 2). Participants were then contacted individually by telephone one week later to confirm participation and arrange an interview time and location. In each case, participants chose to be interviewed within their place of work, except for one participant in Case 2 who requested a meeting off-site at a local coffee shop. During the telephone conversation the participants were asked if they had any questions about the research or the documents they had received,

\section{During the interview}

At the commencement of each interview signed consents were collected and permission was sought (and granted) to record the interview. On average the interviews took between 60 and 90 minutes, although in three instances, interviews extended to 2 hours. The researcher took notes throughout the interview regarding any points of note and perceptions about the participant and interview process. In two instances, interviews were carried out in close proximity to other members of the teams and the researcher was able to informally observe interaction between team members. At the conclusion of the interview participants were offered the opportunity to add any additional information they thought relevant to the conversation.

\section{After the interview}

Following each interview, notes and observations were written into the contact sheets developed for each interview participant (see Appendix 3). These types of field notes provide an ongoing commentary about the research (Van Maanen, 1988 in Eisenhardt, 1989). In all but three instances, interview tapes were transcribed within one week of the interview taking place. The three remaining tapes were transcribed within two weeks. Special notations were made to denote emphasis, humour, pauses, and other such events made during the interviews. The researcher also noted any questions or 
required clarification arising from the transcripts. A transcript of the interview was sent to the participant by email, requesting that the transcript be checked for accuracy and asking for any questions or issues to be forwarded to the researcher. Three responses confirmed the transcript was accurate and another indicated that a small part of the transcript did not correctly portray the participant's opinion about the particular topic. In this instance, a note was made on the transcript and the email was retained and added to the material for analysis. No responses were received from the other participants.

In addition to the interview data gathered in Phase 1, secondary data was collected from web-based sources, and other documentation obtained through the participants.

\section{Data analysis}

Data analysis was acknowledged as a critical stage for the researcher and was complex due to several factors. Firstly, the research involved several cases which were being undertaken concurrently. Due to the availability of individuals involved in each case and time required for analysis, the researcher was often involved in collecting data from one case whilst undertaking preliminary analysis of data already collected from other cases. Secondly, the sequential phasing of the data collection introduced interdependency between each of the phases. For example, in order to confirm the design of the Phase 2 survey, the researcher needed to have completed Phase 1 data collection in at least one of the cases. This level of complexity required the researcher to observe strict procedures to guide data analysis.

The process of analysis enables the researcher to make sense out of the data (Creswell, 2003). Creswell states that during the first stage of analysis the researcher must prepare the data for analysis by reading through the data to gain a general understanding of what is contained there before undertaking detailed analysis and coding. Miles \& Huberman (1994) discuss the problems associated with data overload and data retrieval and the challenge that a researcher faces in making sense of the data. In this respect, they identify the prior development of conceptual frameworks and research questions as the best form of defence (Miles \& Huberman, 1994). The conceptual framework developed in the early stages of this study provided the researcher with an overarching pathway through the research process, but also allowed emergent issues to be explored as they arose. 
Early analysis can enable the researcher to traverse between thinking about the data and the development of new collection strategies; to identify and correct "built in blind spots"; and to generate interim reports (Miles and Huberman, 1994). Early analysis also enables a researcher to make any required adjustments to data collection instruments. Eisenhardt (1989) states that the adjustment of data collection instruments can enable the researcher to probe emergent themes.

\section{Early analysis techniques}

In this research, contact summary sheets were completed for all interview participants. The contact summary sheet is a useful tool to enable the researcher to consider and make sense of the contact with a participant. Miles \& Huberman (1994) recommend completion of the contact summary sheet no later than the day following the interview. While the researcher endeavoured to complete these on the same day or the day following the research, this was not always possible and in some instances time restrictions meant that the contact summary sheet was not completed until several days after the interview was conducted. However, the notes that had been taken during the interview were always re-read and additional thoughts added immediately following the interview and, although not formalised immediately through the contact summary sheet, this initial review helped to ensure that important points were not lost. The summary sheet incorporated points and ideas relating to each of the key areas of the research, as well as identifying emergent themes and general conclusions. The summary sheet was stored in the case study database held in NVIVO and reviewed after the full interview transcript had been completed.

Document summary forms were used to summarise secondary data (see Appendix 4). The amount of secondary data collected in this study was limited, in some cases due to the confidentiality of the projects in question. The use of the summary form helps to put documents in context and enables the researcher to consider its significance (Miles \& Huberman, 1994).

The penultimate stage of early analysis involves the coding of data. Coding represents a key data management tool for researchers (Pare \& Elam, 1997). Coding involves taking data in its various textual or visual forms and organising it into chunks (e.g. words, sentences, phrases, and paragraphs) that can be categorised (Creswell, 2003). Miles and 
Huberman (1994) define codes as tags or labels that can be applied to the data chunks. The coding process used in this study was supported through the use of NVIVO software (version 8), a software package specifically designed by QSR International for the analysis of qualitative data.

Coding was applied to all the qualitative data gathered in the study: interview transcripts, contact summary sheets, secondary data, and document summaries. Initial coding was developed prior to data collection. This coding was derived from the conceptual framework developed at the outset of the study. The framework identified a number of factors from the extant literature that were considered to be of potential importance to this research. These codes were then used to undertake initial analysis of the data. Further analysis of the data identified that a greater range of codes were required to further break down factors identified in the conceptual framework, and to accommodate emergent themes and ideas identified through data collection. The analysis process also identified that data was often relevant to several codes, and in these cases, the data were appended to multiple codes.

During the coding process, the researcher was frequently struck by potential relationships between data and themes, as well as by ideas about various aspects of the research that might be useful to consider during the analysis process. To avoid a proliferation of paper notes and to ensure that all thoughts were captured inside the research databases, these were added as memos and a relationship was made to the interview or document to which they referred. Memos are essentially conceptual in nature in that they can be used to tie together discrete chunks of data and are of the most powerful sense-making tools available to the researcher (Miles \& Huberman, 1994).

The conclusion of the analysis of Phase 1 data enabled the researcher to complete two significant tasks. Firstly, the researcher produced a high-level, descriptive summary of each of the case studies. The summaries contained the key themes pertinent to each study, and displayed both narrative data and the integration of data through tables, matrices, and bar-charts. This enabled the data to become easily accessible in terms of presentation (Miles \& Huberman, 1994). Secondly, the researcher used the analysis from Case 1 to refine the design and content of the survey that was to be used in Phase 2 of the research. 


\subsubsection{Phase 2: Quantitative and qualitative data collection and analysis}

The purpose of Phase 2 of the research was to gather more in-depth data relating to the main themes of the study, to generalise the findings across the study population, and to gather social network data. To facilitate these aims, data were gathered through the use of a survey (see Appendix 5). Initial development of the survey was undertaken prior to commencing data collection in the pilot study, based on factors identified from the literature and encapsulated within the conceptual framework. The survey was adjusted following conclusion of the collection and analysis of Phase 1 data from Case 1. The adjustments included minor formatting changes to the survey's presentation. Two questions were removed from the survey as they had proved difficult for respondents to complete.

The survey was designed to maintain the confidentiality of each participant and a code was assigned to each survey sheet prior to dissemination. This code was recorded against the participant's name in the central database of participant information developed by the researcher.

At this stage, the researcher had only limited knowledge of participants' access to technology and little understanding of their technical expertise. Accordingly, the survey was printed into an A4, 4 page format, and sent to each participant by post. A stamped addressed envelope was included with the survey in order for participants to easily return their completed documents. The survey included a return-by date and one week prior to this date a reminder email was sent to all participants who had not yet responded. Approximately half the surveys were received by the stated return date. Two days after the stated return date, phone calls were made to all participants who had not responded. In most instances the participant was available and promised to complete and return the form. In one instance, a participant in Case Four indicated he would not be completing the form as he did not wish to participate in the research. Further phone calls or emails were sent to individuals who still did not respond. Although timeframes varied between projects, it took an average of about two months to 
receive completed surveys. The overall response rate to the survey was $97 \%{ }^{5}$. Individual case study site response rates are shown in Table 20.

Table 20: Phase 2 survey response

\begin{tabular}{|c|c|c|c|}
\hline Case & No. of surveys issued & No. of surveys received & Response rate \\
\hline Case 1 & 12 & 12 & $100 \%$ \\
\hline Case 2 & 10 & 10 & $100 \%$ \\
\hline Case 3 & 15 & 14 & $93 \%$ \\
\hline Case 4 & 21 & 20 & $95 \%$ \\
\hline Total & 58 & 56 & $97 \%$ \\
\hline
\end{tabular}

\section{Data collection}

The survey collected several types of data including a small amount of demographic information and data collected through open and closed questions. The survey also included questions relating to the SNA aspects of the study. Although the overall contents of the survey remained the same for all cases, the network-related questions were case specific in that they required the participants to identify the team members with whom they interacted. Accordingly, a separate survey was created for each case incorporating the names of each of the team members participating in the case. Use of the same format (apart from names of individuals) enabled cross analysis of the research data and assisted generalisability of the study.

\section{Demographic information}

The researcher had already received some demographic information when the participants had been identified by team leaders; therefore a limited amount of demographic information was requested from participants. Information requested in the survey included an individual's tenure in their parent organisations; position title; whether they had been part of their respective collaboration for the entire lifetime of the endeavour and, if not, when they had joined their team.

\footnotetext{
5 It should be noted that while this appears to be a very high response rate, participants had in fact, agreed to participate in the study at the outset. This was largely to ensure that comprehensive network data could be collected. Had participants declined to participate at the outcome, then the project in which they were involved could not have formed part of this study.
} 
Responses to the demographic information contained several errors: some participants had omitted details; others included the name of their parent organisation, rather than their tenure within the organisation.

\section{Social network data}

Wasserman and Faust (1994) identify the survey as the most common method of social network data collection. Surveys provide quantitative SNA data that measures the structural properties of social networks (Carrington, Scott, \& Wasserman, 2005). In this study, the social network questions gathered data relating to both network configuration and interactions (ties) within the network. This resulted in the collection of data about the individual interactions between team members as well as data relating to the overall network structure. The data provided both visual and quantitative measure of how knowledge is actually shared within each case. The resulting networks can be directly compared to the formal structure of each case.

The survey adopted a roster format whereby all case participants are known to the researcher and their names are included on the survey to enable participants to select those individuals with whom they interact. The roster format was chosen as it helps to ensure that all ties are documented, and is less reliant on the recall of the participant. Specific network questions were developed to identify information flows and communication within the case and to determine the existence of multiple ties between participants.

\section{Closed questions}

The second part of the survey asked participants to respond to a number of questions using a scale of 1-5, where 1 represents "Strongly Disagree" through to 5, "Strongly Agree". Questions were loosely grouped into four sections based on the key themes of the study. The first section requested information relating to the formal structure of the collaboration, the existence of mandated processes and procedures, and decision making; the second section focused on factors relating to SC; the third section explored individual knowledge sharing behaviours; and the final section related to ICT. The purpose of the sections was to indicate to participants the underlying purpose of the questions, rather than to act as formal constructs. 


\section{Open-ended questions}

The survey also contained three open-ended questions. These questions required participants to respond using their own words. The first question asked participants to describe how they defined knowledge. The second question asked participants to list the three greatest challenges that, in their opinion, were faced by participants engaged in inter-organisational collaboration. The third question required participants to identify their perceptions of the three key barriers to knowledge sharing in inter-organisational collaboration.

\section{Data analysis}

Both qualitative and quantitative data was collected in Phase 2 and, accordingly, analysis was undertaken separately.

\section{Quantitative data: Social network data}

Analysis of the SNA data was undertaken using two specialist software applications, UCINET $^{\odot}$ and Netdraw ${ }^{\odot}$ developed by Analytic Technologies. These are two of the most commonly used applications for the analysis of SNA data. UCINET $^{\odot}$ enables quantitative analysis of the data to be undertaken, while Netdraw ${ }^{\odot}$ supports the visualisation of the data into sociograms, often referred to as network maps (see Figure 19, p. 96). Quantitative analysis of the network data is fundamentally different to that undertaken in standard quantitative analysis (Wasserman \& Faust, 1994). SNA data analysis focuses on ties between actors and underlying measures pertaining to the network structure such as cohesion and centrality.

The data was entered into UCINET $^{\odot}$ using text files in the form of nodelists and VNA files (see Appendix 6). The edgelists comprised data relating to the participant (actor) and the VNA files supplied attribute data such as organisation and seniority. This data was then converted to sociograms using NETDRAW ${ }^{\odot}$. A number of measures were calculated on the data including density and distance and network centralisation. A number of analyses were also conducted at the actor level, including the centrality measures for actors. In addition to these quantitative measures, sociograms of each case network were created. These sociograms enable the researcher to quickly identify 
specific aspects of a network that can be further explored through the quantitative measures outlined above.

\section{Quantitative data: Closed questions}

The second stage of analysis focused on the closed questions contained in the survey. The data obtained from these questions was analysed using SPSS@ 14.0 for Windows. SPSS $($ ) was used to obtain descriptive statistics, and the ordinal data entered was transformed to nominal data by recoding participants responses. The categories 5 (Strongly Disagree) and 4 (Disagree) were recoded to 2. The neutral response indicated by 3 was recoded to a 0 , and responses 1 and 2 (Agree and Strongly Agree) were recoded to 1. Data was presented graphically using bar charts that show the response rate, mode, and variance for each question ${ }^{6}$.

\section{Qualitative data: Open questions}

The final stage of analysis of the survey involved the open-ended questions. The responses for each question were organised into matrices. Thematic analysis was then conducted on each matrix using keywords derived from the conceptual framework and analysis conducted in Phase 1.

\subsubsection{Phase 3: Qualitative data collection and analysis}

The purpose of Phase 3 data collection was to further explore the largely quantitative data gathered during Phase 2, and to drill down more deeply into this data. Phase 3 enabled the researcher to explore the specific experiences and beliefs of individuals and to derive a more in-depth understanding of their behaviours and perceptions, particularly in relation to their identified positions within the case networks.

\footnotetext{
6 The researcher acknowledges that more extension analysis of this data could have been undertaken. However, the analysis undertaken met the needs of the study as described in the research questions and objectives. Coupled with the extensive nature of data collection, the complexity of the SNA and time spent to conduct both within case and cross analysis precluded more complex analysis of the data collected in this section. Further, more extensive analysis is expected to be undertaken at the completion of this thesis.
} 


\section{Data collection}

Data were collected through a series of one-on-one semi-structured interviews similar to those carried out in Phase 1. In Phase 3, participants were purposively selected based on three factors: (a) their placement within the network map (for example, did an individual play a very central role in the network that required further exploration); (b) that they had not been part of the interviews conducted in Phase 1; and (c) where possible they represented an organisation that had not been part of the Phase 1 data collection process. These criteria were met for Cases 1, 2 and 3, but were not able to be achieved in Case 4. Participants in Case 4 were located throughout New Zealand and due to participant availability and time and cost considerations, interviews were only able to be conducted with Wellington-based participants. In total, Phase 1 and 3 interviews produced 208 pages of single spaced transcripts.

The collection of data followed the same format outlined in Phase 1, including the signing of a consent form, the recording of the interview and return of the transcript for checking by the participant. Table 21 summarises the key details of each case including the total interviews undertaken in Phase 3 of the study.

Table 21: Phase 3 Data Collection

\begin{tabular}{|l|r|r|r|}
\hline Case & $\begin{array}{r}\text { Total number of } \\
\text { organisations }\end{array}$ & $\begin{array}{r}\text { Total number of } \\
\text { participants }\end{array}$ & $\begin{array}{r}\text { Interviews carried out } \\
\text { in Phase 3 }\end{array}$ \\
\hline Case 1 & 2 & 12 & 3 \\
\hline Case 2 & 5 & 10 & 2 \\
\hline Case 3 & 7 & 14 & 3 \\
\hline Case 4 & 8 & 20 & 2 \\
\hline Total & $\mathbf{2 2}$ & 56 & $\mathbf{1 0}$ \\
\hline
\end{tabular}

\section{Data analysis}

Interview transcripts were entered into $\mathrm{NVIVO}^{\odot}$ and coded in alignment with the codes identified during the analysis of Phase 1 data. Analysis of the data confirmed some of the emergent themes that been identified in earlier stages, and also introduced some new elements. Accordingly, some original codes were expanded in line with the additional data, whilst a small number of new nodes were added. The completion of this analysis enabled the development of the final coding tree shown (see extract in Appendix 7). 


\subsubsection{Integration of the data analysis}

To complete the within-case analysis, it was necessary to consider the findings within each phase of the study. For example, how did participants' comments (derived from interviews), their position in the network (derived through SNA centrality measures), and their perceptions about trust levels (derived from closed questions within the survey) combine to provide an integrated view of the influence of trust in each case.

Although some integration of the data had been undertaken throughout the course of the analysis - for example, qualitative data had been integrated through the use of NVIVO ${ }^{\odot}$ data integration at an overall level was still to be undertaken. It was not possible to import social network data or the data derived through the closed question section of the survey into $\mathrm{NVIVO}^{\odot}$, neither was it possible to import the qualitative data in UCINET $^{\odot}$. In some instances, memos were created in $\mathrm{NVIVO}^{\odot}$ to hold textual summaries of the findings from the SNA data and these were coded to alert the researcher to re-examine data collected in different phases before final conclusions were drawn. Therefore, to a great extent, this was an iterative manual process and the continual cross-referencing of the data collected in each phase helped the researcher to draw conclusions about the research.

\subsubsection{Cross-case analysis}

The use of multiple cases adds to the validity and generalisability of research findings (Miles and Huberman, 1994). The adoption of a case selection strategy, that is, replication logic, is also advantageous (Eisenhardt, 1989; Yin 1994). Miles and Huberman (1994) define cross-case analysis as the process through which patterns and relationships between different cases are identified. The purpose of the cross-case analysis was to identify similarities and differences between the cases through the application of pattern matching. Pattern matching was also used at the earlier stage of analysis where each case was analysed against the preliminary codes identified from the conceptual framework. The cross-case analysis adopted in this research enabled a systematic review and comparison across the four cases.

Miles \& Huberman (1994) suggest three methods of conducting cross-case analysis: (1) by identifying categories or particular areas of interest and comparing within-case similarities with cross-case differences, (2) by selecting pairs of cases and listing the 
similarities and differences between each pair, and (3) by dividing the data according to the nature of data source. The researcher adopted the first strategy by categorising the characteristics of each case, for example type of approach, and then identifying the differences across the four cases. Secondly, the cases were compared based on the factors identified through the conceptual framework and emergent factors derived through the within-case analysis. The researcher also adopted the third strategy and divided data into qualitative and quantitative sets on which cross-analysis was then conducted. The nominal data gathered through the survey was converted to bar-charts during the within-case analysis and these charts were extended across the four cases. Aspects of the qualitative data had been conceptualised through within-case matrices on which thematic analysis was conducted; these matrices were extended to incorporate findings across all cases. Miles \& Huberman (1987) note that this latter strategy enables the researcher to gain unique insights into the data both through corroboration of findings and the ability to reconcile conflicting findings. In addition to the use of bar-charts and matrices, the researcher also developed scatterplots to map relationships between factors. For example, a scatterplot was used to identify the relationship between "frequency of interaction" and the "physical proximity" of case participants.

Cross-case analysis of the social network data was undertaken to identify patterns of the networks and actors and to consider these against the characteristics of each network. These patterns were depicted through the use of sociograms. The characteristics of the within-case networks were compared across each of the cases to identify patterns of similarity and to identify differences. These findings were considered in the broader context of the qualitative data relating to the main themes of the study.

\subsection{Summary of Chapter 3}

This chapter has presented epistemological and methodological considerations of the research process, and the specific research design aspects of this study. The adoption of the post-positivist paradigm supports the objectives of the research, and reflects the researcher's view of the world.

The research methodology section includes discussion of the benefits of case study research and the use of multiple case study. A multiple case approach is justified as an appropriate methodology for undertaking this research. 
This chapter also discusses mixed methods design, and outlines the researcher's choice of a mixed methods approach incorporating the use of qualitative and quantitative research methods, with a predominantly qualitative focus. The concept of SNA was introduced.

The chapter outlines the specific research design guiding this research and the data collection and analysis undertaken in each phase of the research. The chapter closes by outlining the cross-case analysis strategy employed by the researcher.

The next chapter presents the first Case in the study. 


\section{CASE 1 RESULTS}

This chapter presents Case 1, the first of the four cases in this study. The results of the data have been analysed through the development of tables, figures, and matrices. Some of these are included in the Case; others are included in the Appendices.

The chapter is divided into five main sections:

- The first section presents an introduction to each Case; it outlines the Case's purpose, the structure of the inter-organisational team, and details of the Case organisations and participants as well as any formal processes that pertain to the Case.

- The second section focuses on participants' perceptions of knowledge.

- The third section discusses the knowledge sharing activities of the interorganisational team.

- The fourth section discusses the availability and use of ICT tools within the Case.

- The fifth section focuses on SC. It presents the results pertaining to the individual factors relating to the relational and cognitive dimensions of SC. The structural SC results, obtained using SNA, are also presented.

The chapter concludes with a summary and discussion of the key findings of this case.

\subsection{Overview of Case 1}

This section provides an overview of structure, purpose, and activities of Case 1. The section also documents the number of participating organisations, a breakdown of the members from each organisation, and the phases of the research in which they participated. 
Case 1 is based in the New Zealand (NZ) education sector, whose services are provided through seven key organisations, two of which participated in this case; for reasons of confidentiality they are referred to Edu1 and Edu2 $2^{7}$.

Case 1 represents an inter-organisational collaboration that is undertaken within a broader work programme between Edu1 and Edu2. The broader work programme focuses on the collection, analysis, and release of specific aspects of New Zealand educational data. Each participating organisation is responsible for the collection of particular aspects of the educational data. These details must then be shared in order for each organisation to have a complete understanding of the segment of the New Zealand educational environment in which they are engaged. The purpose of Case 1 is to deal with a subset of the overall data, and to share and co-ordinate the release of this data to the general public. The nature of the data is such that it attracts significant media interest and, because of this, both Edu1 and Edu2 view the release of this information as a high risk exercise.

In Case 1, the details of the educational data change each year, therefore the collaboration is an annual occurrence that generally lasts for approximately two months, and follows the same process as shown in Figure 21.

Each year, Edu1 collects a range of educational data from a number of educational institutions throughout New Zealand. Edu1 collates the data and, using a statistical analysis software application, performs a number of analyses to derive the specific information they require. The data is then shared with Edu2 who performs further analysis on it, based on their organisation's requirements. Edu2's analysis is performed using a different statistical analysis software application. During this time, individuals from each organisation collaborate to discuss and agree to aspects of the data prior to its release to the public.

\footnotetext{
${ }^{7}$ Due to the small size of the New Zealand public sector, specific details relating to each organisation have been omitted in order to protect the confidentiality of both organisations and participants
} 


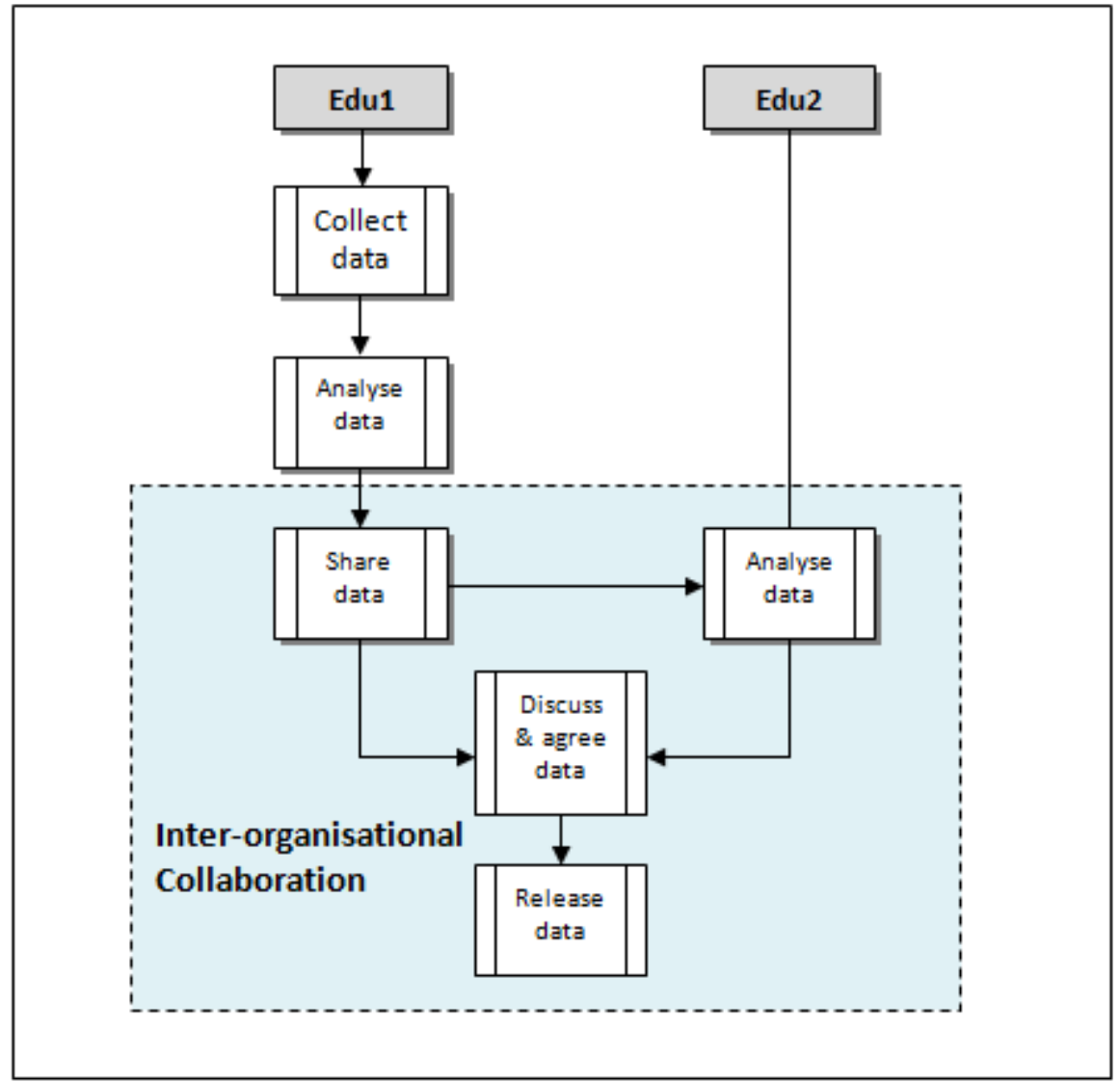

Figure 21: Case 1 Process Diagram

This collaboration is conducted under the auspices of a Memorandum of Understanding (MOU) that exists between the two organisations. This MOU is a high level agreement that sets out the individual roles and responsibilities of each organisation, and outlines guidelines relating to interaction between them. The MOU provides direction at a strategic rather than an operational level and, in relation to this case specifically, there are no formal guidelines, project charter, written agreements, or processes and procedures that define how operational-level work will be carried out.

All of the activities undertaken within the collaboration are considered to be "businessas-usual" activities. As such, there is no formal funding or resource allocation assigned to the collaboration and all activity is conducted as part of the day-to-day activities of individuals.

A total of 12 individuals across the two organisations are involved in this Case. Nine of the team members are employed by Edu1, and the remaining three by Edu2. Although both organisations categorise this as an inter-organisational collaboration, there is no formal structure that brings Edu1 staff and Edu2 staff together into a single inter- 
organisational team. Rather, the two organisations maintain their existing team structures and operate independently within the Case, as shown in Figure 22.

The inter-organisational team does not have a designated leader. The members of Edu1 report to Matthew ${ }^{8}$, who is the most senior member of the Edul team engaged in the collaboration. The exceptions to this direct reporting line are Andy and James, who sit outside of Matthew's direct team, but do become involved in the collaboration.

In Edu2, the two team members report to Barbara, the senior member of the team. The structures within each organisation reflect the normal reporting structure for day-to-day activities outside of this case. In the inter-organisational context, communication between the two organisations is conducted through Matthew and Barbara.

Both Matthew and Barbara have extensive experience and tenure of service within the education sector. Prior to taking up his role at Edu1, Matthew worked at Edu2 for ten years in a research-focused position. He has extensive experience managing projects with multi-disciplinary teams and has been involved in several inter-organisational collaborative endeavours. Barbara has been with Edu2 since 2004, prior to which she spent six months at Edu1. As a result, both Matthew and Barbara have considerable experience within the sector as well as within and across both organisations.

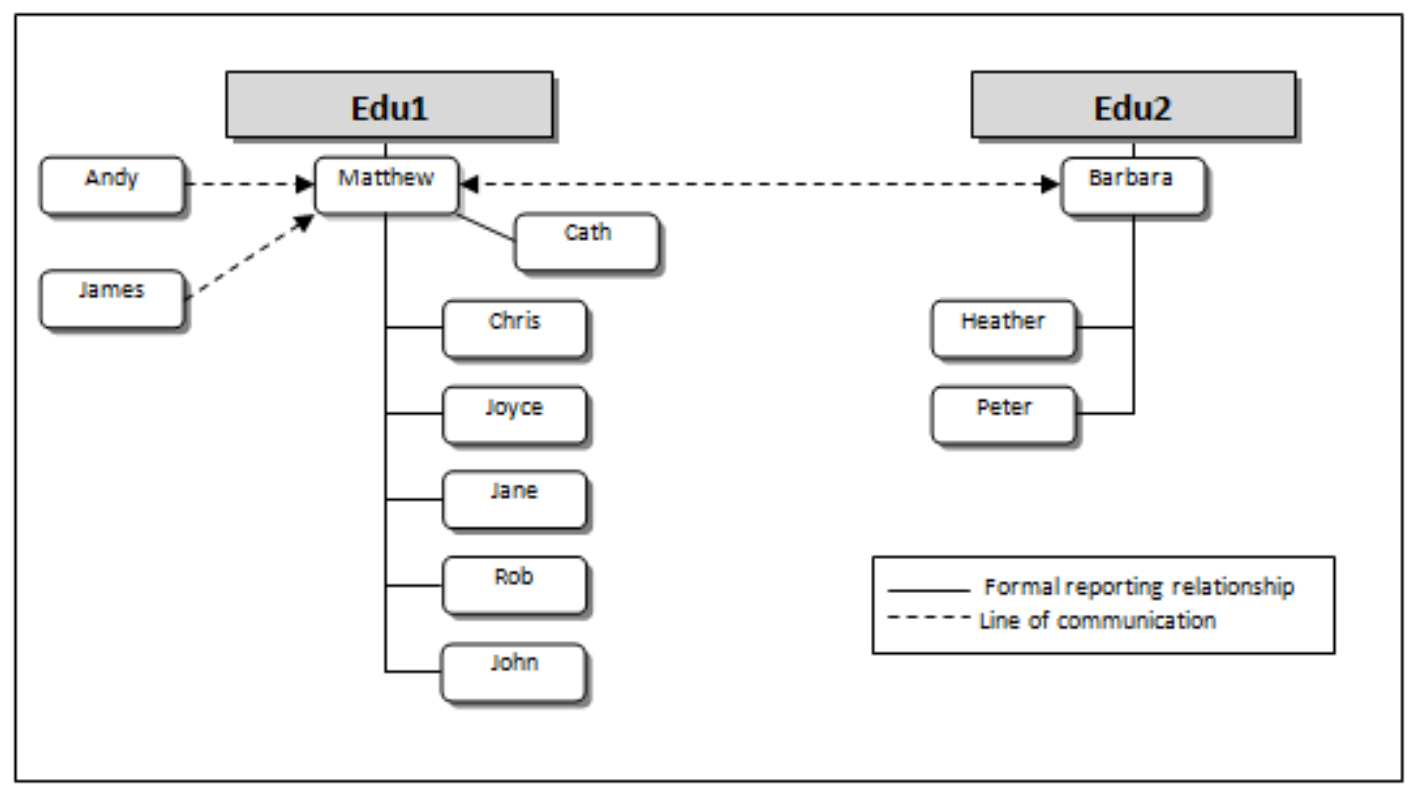

Figure 22: Formal collaboration structure

${ }^{8}$ The use of pseudonyms has been employed to maintain the anonymity of participants. 
Other participants are employed in a range of policy, research, and information-related roles. They occupy a range of levels within their respective organisational hierarchies, from administrative to senior roles. Participants who have been employed in their current roles for more than twelve months have been involved in this Case before, due to the fact that it is an annually recurring collaboration; therefore they have multiple experiences of involvement in the case. Participants' average tenure within their individual organisations is approximately $5.21^{9}$ years. The average tenure of staff across the public sector as a whole is 8.2 years as at 2009 (SSC, 2009). A breakdown of the roles and tenure of Case participants, and their participation in specific phases of the research is included in Appendix 8.

The nature of the collaboration is such that although members of the group do meet face-to-face, these meetings are generally outside the context of this Case and are more often concerned with the wider programme of work that is being undertaken by the two organisations. Thus, this Case is characterised as a virtual collaboration whereby the participants rarely or never meet face-to-face for the specific purpose of the collaboration. The specific characteristics of Case 1 as detailed above are summarised in Table 22.

Table 22: Case 1 Characteristics
\begin{tabular}{|l|l|}
\hline Characteristic & Case 1 \\
\hline Physical Type & Virtual \\
\hline Number of Organisations & 2 \\
\hline Number of Individual Participants & 12 \\
\hline Duration of Collaboration & 2 months \\
\hline Frequency of Collaboration & Annual \\
\hline Allocation of staff resource & Part-time \\
\hline
\end{tabular}

\subsection{Perceptions of knowledge}

At the outset of the Case, participants were asked to define knowledge, and its role within the Case. The purpose of this request was to encourage individuals to actively consider knowledge and enable the researcher to identify similarities and differences

\footnotetext{
${ }^{9}$ This figure is based on the tenure of 7 participants who responded to this question, and is skewed by a single participant with a tenure of 16 years.
} 
between individuals' perceptions, and to help determine the value and role that knowledge plays in the Case. A full list of participants' responses is included as Appendix 9.

Some participants, particularly those who were engaged in the analysis of raw data, refer to the knowledge hierarchy of data, information, and knowledge:

The insights and conclusions gained from analysing information. A continuum of data, information and knowledge.

Building on this perception, other Case 1 participants extend this thinking to refer to knowledge as information that has been further processed to contain meaning and context, and that could be applied or used:

A set of understandings or strategies about the meaningfulness of information, and the context in which it can be used which are dynamic and mutually reinforcing and lead to better understandings or application of practices to solve significant issues or learn about a particular phenomenon.

Information of which someone is aware, has an understanding of and acts on for specific purposes - such as performing their job.

Some participants, notably those in less senior roles, suggest that knowledge is something that is discussed and used at more senior levels, and in fact, their role is to supply the information that would lead to that knowledge:

We [analysts] supply the data, the information. Then they [management] discuss it and ask questions. All we do is supply the raw facts.

Case 1 participants confirm that use of the term "knowledge" is not common within the inter-organisational team, or within their respective organisational teams. They are more comfortable discussing information, and are more easily able to identify the role of explicit knowledge in the inter-organisational collaboration. Participants place considerable emphasis on ensuring that the data and information is correct. This data forms the basis of what is derived and released as information to the Minister and to the general public, therefore any errors in the data can lead to errors in the information that is released. 


\subsection{Knowledge sharing activities}

At its most basic level, participants regard this collaboration as a relatively simple transfer of information whereby the explicit data collected by Edu1 is forwarded to Edu2. To facilitate this transfer, the data are copied onto a CD and manually delivered to the team at Edu2. However, there are a number of activities that occur over the lifetime of the collaboration that support the data transfer. These activities relate to the analysis of the data; issues relating to the use of specific terminology; interpretation of the data; and how the data will be used and shared by the organisations.

The majority of sharing occurs around information. Knowledge is considered to be almost a by-product of the collaboration and knowledge sharing is something that occurs if team members have difficulties understanding the data. As Heather states:

Quite often what we do is in the first instance we will share information ... and go back to them [Edul] if we don't understand ... [knowledge] it's all stuff that you know somewhere but I suspect most of it is in their heads.

Matthew proposes information sharing as an "operational" action, whereas knowledge sharing is something that is done at a more "strategic" or "higher" level:

We draft a report and do an analysis [and] we will share it with other people in the team and we will look at the implications and that knowledge sharing is done at a higher level like at a management level or through a specific internal group.

This confirms other participants' views of knowledge sharing as a more tactical or strategic activity. So, in most instances, data and information is determined by operational level staff, and value is derived and added at a senior level.

Members from each organisation (i.e. within Edu1, and within Edu2) report that sharing is actively encouraged within their individual organisational team, and is a regular occurrence. Within these teams, sharing takes place through scheduled and/or informal meetings, through the exchange of email, and through telephone conversations.

Sharing is less evident across the inter-organisational team. At the inter-organisational level, it is not routine practice to share knowledge across the team, particularly knowledge that is more tacit in nature. Rather, inter-organisational sharing is more 
likely to be driven when an aspect of the information, or the sharing process, requires further clarification or assistance. That is not to say that knowledge is withheld; but that knowledge sharing occurs on an ad hoc, as-required, basis rather than a matter of everyday practice.

Other factors that impact on the degree of knowledge sharing include individuals' proximity to other team members, and lack of time and human resources. In relation to physical proximity, individuals from the two organisations are housed in different buildings; therefore there is little opportunity for casual, ad hoc, meetings between participants to occur. Physical proximity is also associated with how frequently individuals interact. The highest number of interactions takes place between individuals located on the same floor of the same building (i.e. within the same organisation) and these individuals are most likely to interact once a day or more. Individuals located in different buildings (i.e. different organisations) indicate they are less likely to interact with individuals in other buildings, and of those individuals with whom they do interact the frequency of the interaction reduced to less than once a week.

Time constraints also influence knowledge sharing activities. Individuals may be working on multiple projects concurrently and these activities can restrict time for sharing. An Edu1 participant states that multi-tasking is an everyday practice for team members:

The team can be working on 15 or 16 projects.

One participant views the pressures caused by lack of resource as a positive influence on sharing:

We're both under-resourced and if we're able to work together we actually increase our resource that way.

Participants confirm that in many cases knowledge sharing activities take place in the context of the wider work programme, through which individuals take the opportunity to liaise specifically on this collaboration. This has resulted in the development of networks between team members that, while not necessarily focussing on the specific aspects of this Case, do contribute to the overall development of relationships between participants. These networks have provided participants with informal opportunities to 
discuss the Case and these opportunities often enable individuals to learn more than through formal meetings. Two-thirds of participants note that they often learn more from an informal chat than through a formal meeting (see Appendix 10). This preference for informal meetings is reflected in the findings relating to participants' choice of communication channel.

\subsubsection{Choice of communication channel for knowledge sharing}

Participants' choice of communication channel for sharing information and knowledge is impacted by three factors: channel availability, the relationship with the recipient, and the nature of the communication.

In Case 1, participants have access to basic communication channels such as face-toface communication, telephone, and email as shown in Table 23. All participants indicate that, where possible, they try to meet face-to-face with others. However, this is not always possible, particularly when communicating with a participant from outside the organisation, where physical proximity is an issue. In these instances, email is most often used to communicate. Only four participants are likely to use the telephone for communication purposes. ICT tools, such as instant messaging, shared workspaces, or other technology collaboration tools are not used by any participants. (The use of ICT tools is explored in the following section).

Table 23: Choice of communication channel

\begin{tabular}{|l|l|l|}
\hline Channel & $\begin{array}{l}\text { Total } \\
\text { Interactions }\end{array}$ & $\begin{array}{l}\text { No of } \\
\text { Individuals }\end{array}$ \\
\hline Face-to-face & 22 & 8 \\
\hline Email & 19 & 6 \\
\hline Telephone & 7 & 4 \\
\hline Shared technology (i.e. shared workspace, electronic collaboration tool) & 0 & 0 \\
\hline Other & 0 & 0 \\
\hline
\end{tabular}

The majority of face-to-face communication takes place between individuals from the same organisation and consists of scheduled and/or informal meetings between two or more individuals. In Case 1, formal meetings for the inter-organisational team are rarely scheduled. There are however, instances of opportunistic meetings, and these often come about through participants' involvement in the wider work programme. For example, the two team leaders may be in a meeting for other work, and take the opportunity to talk about aspects of this collaboration (Case 1) informally: 
We do have regular face-to-face meetings. I mean they happen quite regularly rather than they are regularly scheduled.

Face-to-face communication between other Case participants is also largely informal and opportunistic, unless they work in close proximity to each other. Edu1 team members confirm that they do have team meetings but these are generally in relation to all the work the team is engaged in, rather than just aspects of this Case. Similar meetings occur in Edu2.

The previous section identified that the lack of face-to-face interaction across the interorganisational team is influenced by physical proximity. However, in the Case of Edu2 participants, this also seems to be something of a conscious decision about how knowledge will be shared. Edu2 participants report that, in a formal respect, they are reliant on Barbara (Edu2 team leader) to share information sourced from Edu1. Barbara confirms that she is the official communication link between the two organisations. However, this has not precluded the establishment of informal networks between individual participants within the two organisations. As Edu2 leader, Barbara, points out:

One of the people in my team has very strong linkages and made sure that she keeps in contact with Chris at Edu.

This informal networking is confirmed by Heather (Edu2):

He [Chris] used to come over here and meetings [non-collaboration related] ....and he was quite similar with his line of thinking and stuff.

Discussion reveals that these networks focus on the wider aspects of the work in which individuals are engaged, rather than on Case-specific aspects, but still provide opportunities for participants to communicate about case-related issues.

The nature of the relationship between individuals also impacts on their choice of communication channel. Sixty-seven percent of participants indicate that the better they know an individual, the more likely they are to communicate with them face-to-face (see Appendix 10). In this Case, most participants have already established a relationship, or know of each other through their involvement in the wider work 
programme, therefore in the majority of cases participants have had previous contact with, or are aware of, other participants. However, the relationship with the participant becomes a secondary consideration when proximity is also an issue.

\subsection{The use of Information and Communication Technology}

There is currently no shared ICT infrastructure between Edu1 and Edu2. Each organisation has individual information systems and there are no connections or integration between them.

The results of the exploration of ICT focus on the main uses of ICT in the cases, and a combination of individual, organisational, and sector factors that influence individuals' awareness and use of ICT for knowledge sharing.

The first use of ICT relates to the analysis of the data collected by Edu1. The data is analysed using a statistical software analysis application, SPSS. Once the analysis is complete, the data is shared with Edu2 who conduct their own analysis using alternative statistical analysis software, SAS. The transfer of the data between the two organisations is facilitated through the copying of the data onto a CD which is then hand delivered to Edu2. One participant notes that this manual transfer is due to the extensive size of the data files that are shared, and which cannot be facilitated through any other means.

The second use of ICT in Case 1 is for communication purposes, through the use of both the telephone and email. Email is the most frequently used channel when face-toface communication is not an option, and email is rated the most common method of communication. Participants regard the existence of an audit trail as an advantage of email communication. This is seen as helpful in reducing risk by being able to have clear records of discussions and decision-making. Fifty-eight percent of participants also believe that email communications help build trust between individuals (see Appendix 10).

Several participants prefer email to the telephone, especially if the choice is between leaving a voice message and sending an email:

I'll generally go to email rather than leave a message on the phone. 
I very rarely just email people unless they weren't there, and/or unless it was a complicated and I found it quicker to deal with, you know... like if I needed to write it down, then I would email it.

Therefore if an individual feels it is important to have documentation relating to a communication, then email is viewed as the most appropriate method for facilitating this. It also appears to enable participants to action the item and allow them to move on to other tasks.

Several factors were found to influence an individual's awareness and use of ICT tools. Within this Case, there are no collaborative ICT tools such as bulletin boards or shared drives or networks available to individuals. Case participants confirm that in most cases they do not have any experience of using these types of collaboration tools. In some cases, participants are unaware of what these tools are, or if they are available to them, or if tools of these types are being used in any other part of the two organisations.

We do document sharing and things like that but we don't have any shared access to anything apart from our mutual websites.

Despite the fact that few ICT tools are available in this collaboration, participants believe that the available technology is effective, and are undecided about whether there is any benefit from having access to a greater range of ICT tools (see Appendix 10). The participants demonstrate limited knowledge about initiatives such as the Government Shared Network (GSN) and Shared Workspaces, and how these might be used by their organisation. As members of Edu1 and Edu2 point out:

There is work towards getting something like that [ability to share data via ICT]. It would be a mechanism to share data and be consistent amongst all organisations...but it's not at a point where I've heard enough about it.

"I'm not trying to be disparaging of the technical solution or anything at all, I'm just saying that I wouldn't want to see technology as the answer to something where it is a bit more about engagement between organisations which is not just in our team, it's an enabler for that rather than the driver for that." 
Despite participants' lack of ICT knowledge and know-how, they believe that ICT assists information sharing and agree that both of the organisations collaborating in this endeavour place a strong emphasis on using ICT to share information (see Appendix 10). This suggests that participants acknowledge the value of ICT to share information, and are used to using it within their parent organisation. Consequently, it could be expected that these opinions would positively influence ICT use within the interorganisational team. However, this was not the case and participants were unaware of ICT initiatives both within their organisation and across the sector.

They [Edu2] are pretty slow about getting information like that out.....A lot of people don't know it exists.

In summary, the reasons for the low use of technology in the Case appear to be a combination of individual, organisational, and sector factors. Firstly, individuals demonstrate low levels of personal awareness of ICT tools and how these might be useful within the collaboration. Though proficient in the use of analysis applications and basic communication tools such as email, participants appear to lack knowledge, and in some cases, confidence about wider uses of technology. At an organisational level, few tools are available and a lack of communication from senior leadership about ICT initiatives and tools means that participants are not actively aware of ICT-related advances that might be occurring within the organisation. At a sector level, initiatives such those relating to e-government are not well known to Case participants. While some know about these at a general level, they do not see how they could be linked to the work in which they are involved and view it as something outside the sphere of their own roles.

\subsection{Social capital}

This section outlines the aspects of Case 1 that relate to SC and how it influences knowledge sharing in this Case.

\subsubsection{Relational social capital}

The relational dimension of SC is concerned with the "why" and "when" of knowledge sharing (Huysman \& Wulf, 2004), and studies most often focus on factors such as identity, trust, norms, and obligations and expectations. 


\section{Identity}

Identity is defined as the process whereby individuals identify with other individuals or a social group (Nahapiet \& Ghoshal, 1998), or the extent to which individuals feel a connection to other individuals (Widen-Wulff \& Ginman, 2004).

In this case, the most prevalent identity-related findings were associated with the wider work programme, and the education sector as a whole, rather than in relation to this specific collaboration.

Participants' comments regarding the nature of the collaboration between the two organisations indicate that there is little sense of group identity in relation to this specific Case. In fact, several participants comment that, in their view, there is not a "formal" collaboration as such. As Barbara points out:

The project [collaboration] is more part of an ongoing relationship between [Edu2] and [Edu1] ... the release of the data is just a small part of that.

This is compounded by a lack of formal title, or name, for the collaboration, which means that participants are unsure how to refer to the collaboration, and as a consequence, often refer to the wider work programme and the activities that occur within the wider programme. This raises questions regarding the legitimacy of this venture as a representation of an inter-organisational collaboration. However, discussion with Matthew (Edu1) confirms that, despite some participants' perceptions, the senior leadership of both organisations recognise this Case as a valid instance of an inter-organisational collaboration.

However, while the collaboration suffers from a lack of identity, there is some evidence of a sense of group identity in relation to the wider work programme in which members of Case 1 participants are engaged. This often involves individuals participating in several working groups responsible for specific aspects of the work programme. One participant points out that though the nature of the collaboration is somewhat undefined, overall individuals "seem to be able to work with each other which is a plus rather than a minus". Another notes the value of recognition that is built up when an individual is involved in a number of different groups focused on related matters, for example being a part of a wider team working on related matters. 
The analysis also suggests an association between identity and the nature of the work in which participants are engaged. During the interview process, several participants made reference to their commitment to the education sector and the importance and value of education. For example, Cliff notes that:

You do this [type of work] because you really believe in it....it makes a difference.

Therefore, a sense of identity also appears to be associated with the education sector itself and that participation within the sector reflects members' commitment to the field of education.

Trust

All Case 1 participants agree that trust is an important factor in inter-organisational collaboration and fifty percent of participants believe that trust levels within the interorganisational team are above average (see Appendix 10). Trust is developed through demonstrations of individual competency and through participants' commitment to the collaboration and its value in the wider sector. Trust is influenced by factors at the individual, team, and organisational levels.

In this Case, trust is based on how competent participants perceive the individual to be, rather than how well they know the individual. Competence can be assessed through an individual's demonstration of their capability and expertise observed through interaction with the individual, or an individual's contribution to, or delivery of, case-related outcomes, as noted by an Edu1 participant:

You build up trust over time...by working alongside them you get to know what they do well.

Given that the majority of interaction appears to take place between individuals from the same organisation (rather than across the inter-organisational team), participants find it more difficult to assess the competency of individuals outside their own organisation, and this can affect trust across the team:

I trust everybody here [Edu1] ... but to be honest, the [Edu2] team I don't well enough to say. I don't know how competent they are. 
Edu1 participants confirms that achieving higher levels of inter-organisational trust requires an increased level of interaction between participants from the different organisations:

Having people work together more. Having more [interaction].

I would want people to be contributing ideas, and encouraging people to consult with one another over their work....Getting to know what one another do, what one another are good at, what one another can help with.

Email is viewed by the majority of participants as one method of helping to build trust between individuals, particularly in the absence of face-to-face interaction.

Trust between Case participants is also influenced by an individual's sense of commitment to the field of education. Participants' perceptions of the value of the work mean that they are more likely to trust an individual, than not to trust them. This finding was reaffirmed through participants' identification of knowledge sharing barriers where trust was not considered to be a barrier to sharing.

Participants' trust in an individual can be affected by the level of trust in the individual's parent organisation. Participants from both Edu1 and Edu2 express reservations about the way in which the two organisations sometimes handle information and knowledge that has been shared between the two. One participant relates a story where information had been shared between the two organisations (outside of this collaboration) and the information had been used by the recipient organisation without the permission of the organisation from which the information had originated. In instances such as this, a lack of trust in the organisation can over-ride a participant's trust in an individual. As Matthew notes:

When [Edul] staff saw the fact sheet they came zooming into my office and said what's happening here because they were highly allergic to any information going out before our proper procedures were followed.

Participants confirm that in such instances, while they may trust an individual, concerns about the organisation may lead them to be less open than they would normally be with the individual. 
Trust in the organisation appears primarily focused on risk associated with the release of information to the general public, and to the Minister. The interviews reveal that participants in both Edu1 and Edu2 perceive there to be high risk in the information that they deal with, and that this risk leads them to be vigilant and more likely to display risk-averse sharing behaviour.

It is simply about... trying to manage what information goes out from the organisation, how the public perceives us, the relationship with the Minister.... We don't want the Minister ... to hear things through another party ... without having had first the chance to explore those issues with the Minister [ourselves].

Timing and issues relating to the wider context are also considerations that the two organisations take into account:

The rules of engagement about information that we have been producing have tended to vary depending on the product, the timing and how it sits into what's going in the wider environment.

There was also some evidence of trust issues at an intra-organisational level. Participants from both organisations recount how internal knowledge sharing is sometimes limited or inhibited by decisions made higher in the organisational hierarchy.

I was told that it has to be checked, or the time isn't right to share that information.

There was an incident where there was a high level agreement that they didn't tell anybody about until we were about to push something and it turned out they'd agreed that nothing would be shared.

This finding is confirmed through participants' identification of knowledge sharing barriers, where issues relating to management and leadership decisions are noted by several individuals.

In summary, Case 1 findings relating to trust show that at an individual level trust is primarily based on competency, but also relates to an individual's belief in or commitment to the work in which they are engaged. There are issues of trust between the two participating organisations that can affect sharing within the collaboration 
although, to a large extent, individuals attempt to overcome these limitations. Trust is also an issue at the intra-organisational level, where sharing can sometimes be inhibited by directives from senior leadership.

\section{Norms, obligations, and expectations}

Scarbrough and Carter (2000) posit that obligations and expectations can manifest as commitment at the individual or organisational level. In Case 1, the influence of norms, obligations and expectations is most clearly demonstrated through individuals' identification of factors that motivate them to share knowledge with other team members. Participants were asked to identify the main motivation for sharing knowledge with each of the individuals in the team with whom they had indicated a knowledge sharing relationship (see Table 24).

\begin{tabular}{|c|c|c|}
\hline Motivation & $\begin{array}{l}\text { Total } \\
\text { Interactions }\end{array}$ & $\begin{array}{l}\text { No of } \\
\text { Individuals }\end{array}$ \\
\hline Commitment to the collaboration & 20 & 4 \\
\hline The belief that the recipient will share their knowledge with me in return & 17 & 7 \\
\hline The feeling I am able to help that person & 16 & 6 \\
\hline Trust in the individual & 6 & 4 \\
\hline Recognition of my knowledge and expertise & 5 & 1 \\
\hline \multirow[t]{2}{*}{ Presence of technology that makes sharing easy } & 0 & 0 \\
\hline & 64 & \\
\hline
\end{tabular}

In total, participants identify 64 sharing interactions. The three most common motivations are individuals' commitment to the collaboration (although this was later determined to be their commitment to education in general); the belief that knowledge sharing will be reciprocal; and the feeling of being able to help an individual.

This chapter has already identified the strong sense of commitment that individuals feel towards the importance of the education sector and, by association, their role in the work in which they are engaged. Commitment to the collaboration is driven by a participant's sense of professionalism and dedication to the subject domain as evidenced through the enthusiasm and deep regard for the New Zealand education system as vocalised by an Edu1 member during the interviews:

You choose to [work in education] because you have a particular way of thinking about people and your place in the world. 
This sense of commitment to the field of education (and by default to the collaboration in which they are engaged) influences individuals' activity with other individuals and can assist the development of trust between participants. It also reflects the finding relating to "Identity" which shows that participants' sense of identity is based more on their connection and commitment to the field of education than on the collaboration itself. This type of affective commitment has previously been linked to an individual's identification with an organisation or feeling of emotional connection to the organisation (van den Hooff \& Ridder, 2004). In this Case, it manifests at a sectoral level, and is demonstrated through an individual's commitment to the field of education.

A further example of this aspect of relational SC is in the expectation that knowledge sharing will be reciprocal. In this way, individuals are motivated to share knowledge based on the expectation that they will receive knowledge in return.

Finally, some participants identified that being able to help a fellow team member is influential in their decision to share knowledge. While this can be described as an example of altruistic sharing, it also signifies a subconscious obligation on the behalf of an individual to share with another. This appears linked to the sense of identity and commitment that relates to the work in which the participants are engaged and which provides the shared context within which the Case takes place.

\subsubsection{Cognitive social capital}

Cognitive SC relates to "what" is being shared, for example the purpose and goals of the inter-organisational collaboration. Research in this area has commonly focused on shared language, shared vision, and a sense of shared culture (see Chow \& Chan, 2008).

\section{Shared language}

A lack of shared language both at the team and organisational levels is raised by several Case 1 participants, and cited as a key barrier to knowledge sharing.

The two organisations use different terminology to describe aspects of educational data, which can cause difficulties in communication and sharing: 
We had to spend a bit of time learning what each other means, and because we already had a set of jargon and definitions, we had to sort of invent new ones when working with [Edu1].

These differences relate to very basic language elements. For example, the term "student" is defined and analysed differently within each organisation and this can lead to confusion.

The lack of shared language causes difficulties for case participants and has a negative impact on the time available for interaction between team members. Rather than spending time discussing the outcomes of the results, team members must focus on ensuring that errors that are due to language inconsistencies are avoided and eliminated:

They [Edul] use language we don't and we use language they don't. So that has probably been the most time consuming and most necessary piece of work.

Discussion with participants who have been involved in this collaboration over several years indicates that, despite the acknowledged difficulties that a lack of shared language has caused, there has been no action to address the issue.

The lack of shared language indicates a degree of disjointedness across both the organisations and the education sector as a whole; a single shared language would facilitate ease of information and knowledge sharing between organisations.

\section{Shared purpose and goals}

The purpose of Case 1 is to share and release specific educational data. Though some participants suggest that Case 1 is more an activity within the wider work programme than a discrete collaborative endeavour, they are clear about the goal of the interaction. Therefore, in the context of this case, there is a sense of shared purpose about what is to be achieved, particularly in the case of the respective team leaders of Edu1 and Edu2.

The notion of shared purpose and goals becomes particularly problematic at the organisational level. Though the two organisations are both engaged in activity in the education sector, an Edu2 participants points out there is a clear difference in their respective purposes: 
We have different aims, different focuses, and it is sometimes difficult to understand each other.

Matthew notes that these differences affect sharing within the collaboration and across the greater work programme.

"There's a definite sort of tensions about [the fact that] we have different ways of working and the different things that we end up doing, and sometimes these conflict.

These differences are evidenced through the earlier example whereby the term "student" is interpreted differently by the two organisations and, as such, can lead to confusion including the way in which the data is collected and analysed. Each organisation approaches this differently, and as an Edu2 participant states:

We think our methodology is better and they think their methodology is better.

Though a single example, this reflects a lack of consistency and level of difference that one would not expect to be evident where two organisations are engaged in working within a single educational system, and where data is drawn from the same population. These differences reflect the cultural differences between the two organisations as detailed in the follow section.

\section{Shared culture}

At a team level, neutral or non-response to the culture-related survey questions (see Appendix 10) indicates that fifty percent of Case 1 participants are unsure about culture and its role within the collaboration. Discussion with participants revealed two potential reasons for this lack of clarity. Firstly, other than at a leadership level there is little inter-organisational interaction between participants. Secondly, the fact that nine of the inter-organisational team members are from the same organisation means that in many respects, participants do not feel like they are part of a larger inter-organisational team, and are unlikely to experience specific cultural differences.

However, exploration of culture issues with the team leaders of Edu1 and Edu2 does identify cultural differences at an organisational level, and these are perceived by the team leaders to influence the nature of inter-organisational sharing. These participants 
have worked within both organisations, and are therefore able to contrast the differences in organisational culture; their thoughts and comments on the differences between the two organisations are based on in-depth experience. They perceive the two organisational cultures as quite different, and a significant challenge to overcome, particularly when it comes to sharing knowledge. Edu2 is seen as more open to sharing, whereas Edu1 has traditionally been less open, except at senior leadership levels:

I've been at national meetings where people have said to me it would be really good if [Edul] could be a wee bit more transparent with processes and so on... but I think in the last six months we have been ten times more transparent.

Edu2 is a much more open culture ...whereas here the culture is open in the management team but in terms of going beyond that ... not so much.

Referring to general collaboration and sharing between the two organisations, one participant states that:

Our biggest challenge is language and the different cultures.

However, both participants believe that in respect to this case, the way in which individual participants approach sharing and inter-organisational collaboration does alleviate some of the issues that can arise from cultural differences, and helps to ensure that cultural differences between the two organisations are not a predominant issue for the inter-organisational team.

\subsubsection{Structural social capital}

Structural SC denotes the "who and how" of knowledge sharing. In this research, structural SC has been approached from two perspectives: the formal structure (who reports to whom), and the informal structure (who interacts with whom). The purpose of exploring these formal and informal structures was to compare and contrast the two.

The formal structure was detailed in Section 4.1. It showed that participants have not adopted a formal inter-organisational structure and continue to work within the boundaries of their individual teams, with the respective team leaders providing formal communication links. 
The informal structure was explored using SNA. Participants were asked to identify those individuals from whom they sought collaboration-related help and advice. The data was explored to:

$>$ Provide a sociogram of the informal sharing network in place within the collaboration

$>$ Identify characteristics relating to the configuration of the informal network (network level results)

Identify characteristics relating to the relationships between individuals engaged in the Case (individual level results)

Figure 23 presents the visual network of Case 1. The sociogram depicts the structure of the informal network and the ties between individual actors. Each actor is depicted as a circular node, and the colour of the node denotes the parent organisation to which they belong. Line colour depicts the direction of the tie. One-way ties are shown in blue and reciprocal ties are depicted by red lines. Tie strength is indicated by the thickness of the line, and the associated values which indicate the frequency of contact from both the initiator and the receiver of the tie. A simple example taken from the map shows that Rob and Matthew enjoy a reciprocal tie (thin red line) with each seeking information and advice from the other. The values show that Rob usually seeks advice from Matthew once a week (2.0), however Matthew goes to Rob less frequently at less than once a week (1.0). By contrast, Matthew and Andy indicate a strong reciprocal tie (thick red line); with each indicating that they are likely to consult the other more than once a day (5.0).

The results show that information and knowledge is more commonly shared among participants from the same organisation than across the inter-organisational team. This suggests that individuals within each organisational team work independently, or within their own organisational silos, rather than as a unified collaborative team. In this respect, the informal network reflects the formal structure of the team described by the respective team leaders of Edu1 and Edu2 and shown earlier in Figure 22. The results also show the presence of 10 reciprocal relationships. In reciprocal relationships participants both seek information from, and provide information to, each other. These 
relationships are considered to be an indicator of tie strength between individuals (Hanneman \& Riddle, 2005). Tie strength is discussed later in this chapter.

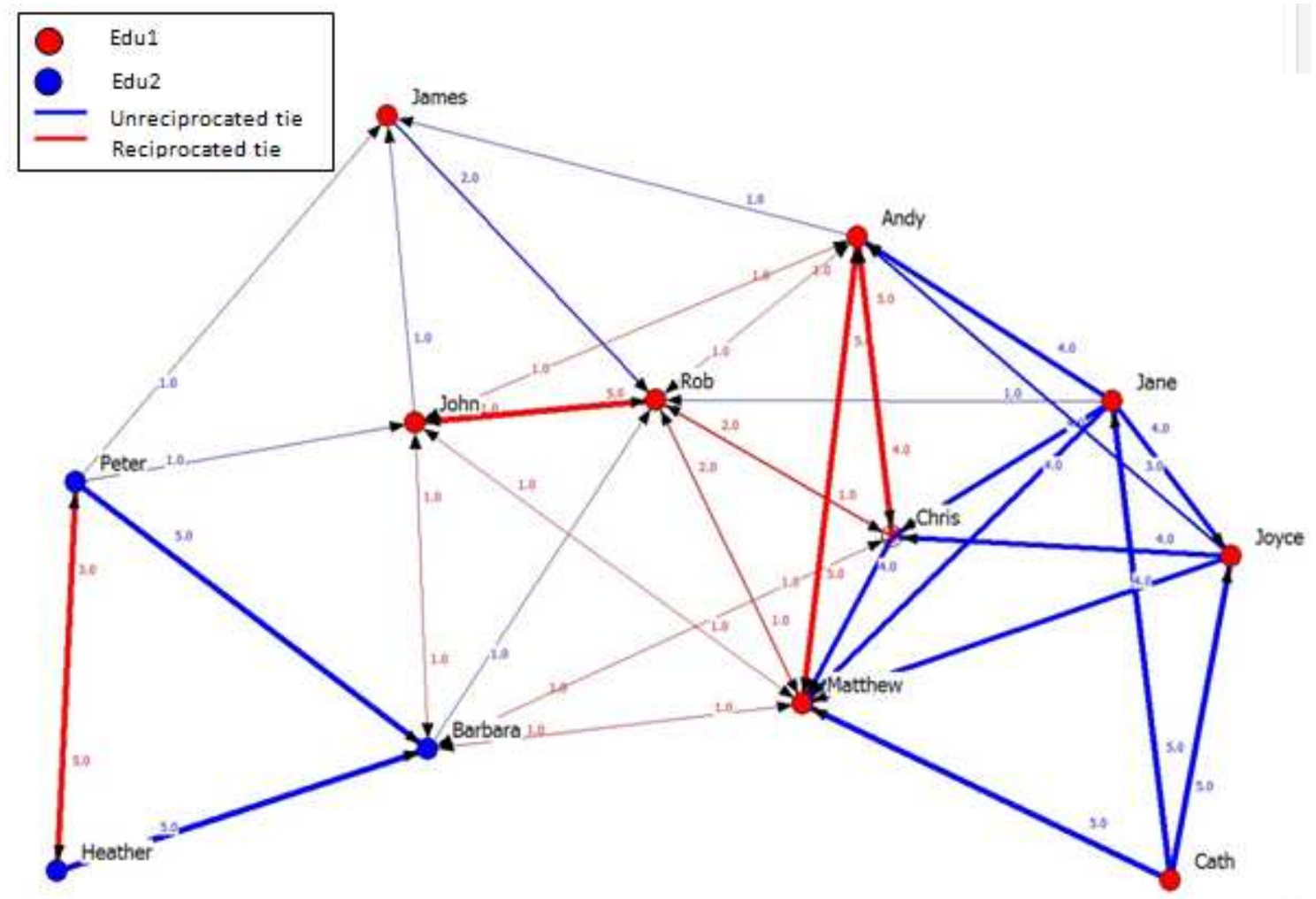

Figure 23: Case 1 informal network

\subsubsection{Network level results}

Network level measures provide an indication of the health and overall connectivity of the network. The network data gathered in Case 1 included the size, density, and distance measures as shown in Table 25. (Extended descriptions of these measures can be found in Chapter Three.) Also included are several descriptive statistical measures derived from the univariate statistics calculated for Case 1.

Table 25: Network level measures

\begin{tabular}{|l|l|}
\hline Measure & Result \\
\hline Size & 12 \\
\hline Total Ties Present & 44.000 \\
\hline Density (Mean) & $33.33 \%$ \\
\hline Distance & 1.5 \\
\hline Std Deviation & 0.471 \\
\hline Variance & 0.222 \\
\hline
\end{tabular}

The network size of 12 indicates the number of participants within the interorganisational team. In networks of this size, it is easier for individuals to get to know 
others than in networks that include a greater number of participants. The network density, or overall connectedness, for Case 1 is $33 \%$. This means that approximately one-third (or a total of 44 ties) of the total possible ties are in place across the network. This is a relatively low density for the network given the small size of the network; the fact that participants indicate that they are familiar with other team members at least by name, but many by sight; and, that this is an annually recurring collaboration, therefore several team members have participated in the collaboration before. For these reasons, it might be expected that individuals would have greater interaction with others and this would be reflected by a higher density measure. The lower density measure might also suggest that task allocation within the collaboration is clearly defined such that in some instances there may not be a need for certain sets of individuals to interact.

The average distance between individuals, or the number of people they would need to go through to gain information if they were not directly connected to that individual, is 1.5. This suggests that despite the low density measure, individuals are easily able to seek information via others when required. Due to the size of the network, and the fact that only two organisations participate in the collaboration, it would be unusual for a higher distance measure to be in place.

\subsubsection{Individual level results}

Three centrality measures were selected to analyse the ties between individuals in the network. The first measure, "degree centrality", was used to measure the number of direct connections an actor has. The second measure, "closeness centrality", was used to measure how close the actor is to all other actors within the network, irrespective of direct connections. The third measure, "betweenness centrality" was used to measure an actor's strategic position within the network. Fuller descriptions of these measures can be found in Chapter 3 .

\section{Degree centrality}

Degree centrality measures the number of direct connections an actor has. A full list of degree centrality degree scores for Case 1 is in Table $\mathbf{2 6 .}$ 
Table 26: Highest and lowest degree centrality scores

\begin{tabular}{|l|l|l|l|r|r|r|}
\hline $\begin{array}{l}\text { Degree } \\
\text { Centrality }\end{array}$ & Actor & $\begin{array}{l}\text { Formal } \\
\text { Role }\end{array}$ & Organisation & NrmOutdegree & NrmIndegree & NrmDegree \\
\hline+ & Matthew & Leader & Edu1 & 36.364 & 72.727 & 72.727 \\
\cline { 2 - 7 } & Andy & Member & Edu1 & 45.455 & 54.545 & 63.636 \\
\cline { 2 - 7 } & Rob & Member & Edu1 & 36.364 & 63.636 & 63.636 \\
\cline { 2 - 7 } & John & Member & Edu1 & 45.455 & 45.455 & 54.545 \\
\cline { 2 - 7 } & Jane & Member & Edu1 & 45.455 & 9.091 & 54.545 \\
\cline { 2 - 7 } & Barbara & Leader & Edu2 & 36.364 & 45.455 & 54.545 \\
\cline { 2 - 7 } & Chris & Member & Edu1 & 36.364 & 45.455 & 54.545 \\
\cline { 2 - 7 } & Joyce & Member & Edu1 & 27.273 & 18.182 & 45.455 \\
\cline { 2 - 7 } & Peter & Member & Edu2 & 36.364 & 9.091 & 36.364 \\
\cline { 2 - 7 } & James & Member & Edu1 & 9.091 & 27.273 & 36.364 \\
\cline { 2 - 7 } & Cath & Member & Edu1 & 27.273 & 27.273 \\
\cline { 2 - 7 } & Heather & Member & Edu2 & 18.182 & 9.091 & 18.182 \\
\hline
\end{tabular}

These measures show a wide variance between the highest and lowest scores of actors, and indicate that some individuals may find it difficult to source information within the Case. To derive greater certainty and clarity about individual measures, degree centrality was analysed further to determine the measure of in-degrees and out-degrees. In-degrees denote the total number of incoming connections, or information-seeking requests made of the individual. Out-degrees denote the number of information seeking requests that the individual makes of others.

Matthew, Rob, and Andy received higher in-degree scores than any other individuals in Case 1. This indicates that their advice is sought more often than other individuals' within the network, and that they may be considered to be experts and/or more knowledgeable than other individuals. Alternatively, they may act as gatekeepers within the network, controlling the flow of information. In social network terms, these individuals are more prominent or prestigious than other individuals. Conversely, Cath, Jane, Peter, and Heather received very low in-degree scores, indicating they are seldom sought out for information and advice. Indeed, Cath was not sought out by any other members.

The highest out-degree scores were achieved by Andy, John, and Jane. Although high out-degree scores can represent an individual's influence within a network, analysis of the Case 1 out-degree scores suggest that in Jane's case job role and tenure may also play a part in the scores attained. As Jane is relatively new to the team, it is surmised that her high out-degree rate is due to the need to gather information to which others may already have access. At the opposite end of the scale, Heather and James scored 
lower out-degree scores than any other members of the team. Further, despite Barbara's leadership role within Edu2, both her in-degree and out-degree scores signify that she does not occupy a key role within the overall network.

From the overall degree scores, it is clear that Matthew and Andy play powerful roles within the network, while Heather and Cath are less well-connected. In Heather's case, this is particularly significant as both her in-degree and out-degree scores show that she has less interaction with others and is somewhat isolated from other participants. Cath's low degree score is most likely due to her role as the sole administrative person within the collaboration. While she does need to seek information from others, Cath's indegree score reflects the fact that it is unlikely that others will go to her for collaboration-related help and advice, and also suggests that the type of help and advice others seek is technically-orientated.

It is also notable that the highest scoring actors are all members of Edu1 and, of these, each has significant experience within the sector.

Network centralisation was also measured as an additional point of reference for the social network data. Network centralisation measures the global or macro level centralisation of the network and is indicative of how unequal the distribution of centrality is in a network or how much variance there is in the distribution of centrality in a network. In this Case, network centralisation was derived from the degree centrality scores as shown in Table 27.

Table 27: Degree Centralisation Index

\begin{tabular}{|l|l|}
\hline Centralisation Index & Result \\
\hline In-degree & $42.975 \%$ \\
\hline Out-degree & $13.223 \%$ \\
\hline Total & $56.20 \%$ \\
\hline
\end{tabular}

The overall degree centralisation index of 56.20 indicates that the network is centralised across a number of individuals. Hanneman \& Riddle (2005) states that the star network with a centralisation index of $100 \%$ is representative of the most highly centralised network. Analysis of these results by in-degree and out-degree shows that the number of information requests that are made is directed at a small group of individuals and is therefore highly centralised. There is less centralisation of those seeking requests and these requests tend to come from across the network, rather than a selected group of 
individuals. Taken as a whole, this indicates that the power of individual actors differs significantly and that those in more central positions are likely to be more positively positioned than others.

\section{Closeness centrality}

Closeness centrality measures the degree to which an actor is close to all other actors in a network (Hanneman \& Riddle, 2005). While degree centrality considers only the immediate ties of an individual, closeness centrality also takes into account indirect ties. Actors with high closeness centrality are able to reach lots of other actors within the network (Kilduff \& Tsai, 2003). Degree centrality represents an individual's local position within a network, whereas closeness centrality indicates an individual's global position. A full list of closeness centrality scores is included in Table 28.

The closeness scores indicate that Matthew holds the strongest global network position, and is closer to other actors than any other actor in the network. The closeness scores also focus on Edu1 team members, and reflect the high degree scores achieved by Matthew and Rob. Once again, Edu2 team members Heather and Peter are distanced from other actors; however, Joyce holds the weakest position in the global network and is somewhat isolated from colleagues. As the calculations are done on directed data (indicating the direction of ties), an overall measure of network centralisation cannot be calculated.

Table 28: Closeness centrality scores

\begin{tabular}{|l|l|l|l|r|r|r|}
\hline $\begin{array}{l}\text { Closeness } \\
\text { Centrality }\end{array}$ & Actor & $\begin{array}{l}\text { Formal } \\
\text { Role }\end{array}$ & Organisation & inCloseness & outCloseness & TotalCloseness \\
\hline+ & Matthew & Leader & Edu1 & 78.571 & 16.176 & 94.747 \\
\cline { 2 - 7 } & Rob & Member & Edu1 & 73.333 & 16.176 & 89.509 \\
\cline { 2 - 7 } & John & Member & Edu1 & 64.706 & 16.418 & 81.124 \\
\cline { 2 - 7 } & Andy & Member & Edu1 & 64.706 & 16.418 & 81.124 \\
\cline { 2 - 7 } & Chris & Member & Edu1 & 64.706 & 16.176 & 80.882 \\
\cline { 2 - 7 } & Barbara & Leader & Edu2 & 61.111 & 16.176 & 77.287 \\
\cline { 2 - 7 } & James & Member & Edu1 & 55 & 15.278 & 70.278 \\
\cline { 2 - 7 } & Cath & Member & Edu1 & 8.333 & 27.5 & 35.833 \\
\cline { 2 - 7 } & Jane & Member & Edu1 & 9.091 & 23.404 & 32.495 \\
\cline { 2 - 7 } & Peter & Member & Edu2 & 9.091 & 22.917 & 32.008 \\
\cline { 2 - 7 } & Heather & Member & Edu2 & 9.091 & 21.569 & 30.66 \\
\cline { 2 - 7 } & Joyce & Member & Edu1 & 10 & 18.644 & 28.644 \\
\hline
\end{tabular}




\section{Betweenness centrality}

Betweenness centrality is often described as a measure of gate-keeping, and is considered to denote a position of strategic advantage and information control (Hawe \& Ghali, 2007). A complete output of betweennness scores for Case 1 is shown in Table 29.

Table 29: Betweenness centrality scores

\begin{tabular}{|l|l|l|l|r|}
\hline $\begin{array}{l}\text { Betweenness } \\
\text { Centrality }\end{array}$ & Actor & $\begin{array}{l}\text { Formal } \\
\text { Role }\end{array}$ & Organisation & BetweennessN \\
\hline+ & Matthew & Leader & Edu1 & 6.545 \\
\cline { 2 - 5 } & Barbara & Leader & Edu2 & 6.409 \\
\cline { 2 - 5 } & Andy & Member & Edu1 & 6.212 \\
\cline { 2 - 5 } & Rob & Member & Edu1 & 6.091 \\
\cline { 2 - 5 } & John & Member & Edu1 & 5.212 \\
\cline { 2 - 5 } & Chris & Member & Edu1 & 2.53 \\
\cline { 2 - 5 } & Peter & Member & Edu2 & 1.545 \\
\cline { 2 - 5 } & Jane & Member & Edu1 & 1.439 \\
\cline { 2 - 5 } & Joyce & Member & Edu1 & 0.985 \\
\cline { 2 - 5 } & James & Member & Edu1 & 0.303 \\
\cline { 2 - 5 } & Heather & Member & Edu2 & 0 \\
\cline { 2 - 5 } & Cath & Member & Edu1 & 0 \\
\hline
\end{tabular}

The results show that there is variation between the low and high betweenness scores. The highest scores are achieved by Matthew and Barbara, and this indicates they may exert a degree of control over information within the network; this is supported by comments received during the interview process which indicated that all information into and out of Edu2 is controlled by Barbara:

I am the contact, everything's supposed to come through me [Barbara].

Yes, everything is channelled through Barbara - we don't really have much contact with them [Edu1] at all.

Andy's high degree score, together with his high betweenness centrality suggests that he is placed in a strategic position within the network and is likely to be highly influential in the flow of information and knowledge. However, unlike Matthew and Barbara who appear to be formal gatekeepers of information, it is likely that Andy plays more of a brokerage role. His high tenure (16 years) also indicates that he has accumulated a wealth of experience that will be highly sought after by others, but that he is unlikely to have to seek assistance from other team members. 


\section{Cliques}

The data were also investigated for evidence of cliques within the network. Cliques represent subsets of the network in which actors are more intensely linked (Hanneman \& Riddle, 2005). A minimum number of three actors is required for a clique to be identified. Three cliques are identified in the network as shown in Table 30. Statistics relating to these cliques are included in Appendix 11.

Table 30: Case 1 cliques

\begin{tabular}{|l|c|c|c|c|}
\hline Cliques & \multicolumn{4}{|c|}{ Members } \\
\hline Clique 1 & Andy & John & Matthew & Rob \\
\hline Clique 2 & Andy & Chris & Rob & \\
\hline Clique 3 & Barbara & John & Matthew & \\
\hline
\end{tabular}

In common with other individual measures analysed in this research, the cliques in Case 1 are dominated by participants from Edu1. The single exception to this occurs in Clique 3 where Barbara (Edu2) was identified as a Clique member.

Clique 1 represents the largest and most dominant subgroup of actors within the Case, and the members of this clique are also notable for their high centrality scores. Each of the two smaller cliques overlaps with Clique 1, through the membership of Andy, Rob, John, and Matthew, who are each members of at least two cliques. Although Barbara and Chris are identified as members of only one of the three cliques, their high betweenness scores mean that they are strategically positioned to the other cliques and are likely to have access to the knowledge shared between members of the other cliques. By contrast, five team members are isolated from all three cliques and this is likely to impact on the extent to which they receive information and knowledge from within the network.

There is some evidence that clique membership may be related to gender, tenure, and organisation. Though almost $50 \%$ of the overall inter-organisational team are female, Barbara is the only clique member who is female, and is the most senior female participant within the Case. In relation to tenure, with the exception of Chris, all other clique members have in excess of 3 years tenure with their respective organisations. All clique members, other than Barbara, are members of Edu1. 
Tie strength

The strength of ties between actors is dependent on the number and types of relationships which a pair maintains, and on the strength of each individual relationship (Haythornthwaite, 1999). Tie strength is important in the assessment of the overall connectedness of actors in an environment and the likelihood that information will flow from one actor to another.

Analysis of the strength of ties between actors in Case 1 ties was based on two factors:

1. Whether the tie was reciprocal

2. The frequency of interaction between the individuals.

The strongest ties are represented by thick red lines (reciprocal with high frequency of interaction), while the weakest relationships are depicted as thin blue lines (one way with low frequency of interaction). Frequency was measured through individuals' reporting of the number of interactions with other team members.

The overall measure of reciprocity for the network is $37.5 \%$. This means that approximately one third of total possible ties are reciprocal, with individuals enjoying a mutual exchange of information and knowledge. However, the remaining two-thirds of the network is characterised by non-reciprocated ties.

The two strongest ties extend between actors in Edu1 (Andy and Matthew; Andy and Chris). These reflect these actors' strong centrality scores and membership within cliques and endorse the dominant role of these actors in the network. Matthew has the highest scores in each centrality category, and can be seen as a powerful member of the inter-organisational team. He is strategically placed within the network and has a high degree of control over the flow of information across the network. Advice-seeking is centred on Matthew, and it is likely that this might result in a degree of overload.

The data also reveals that the tie between Barbara and Matthew is a weak tie. Although these actors enjoy a reciprocal relationship, the frequency of interaction was indicated by both actors as being less than once a week. Thus, their relationship is not as strong as other actors such as Matthew and Andy, who also enjoy a reciprocal tie but have a higher frequency of interaction. This suggests that Matthew and Barbara are likely to 
share more diverse information (Hansen, 1999), and that the relationship does not require the amount of time or emotional intensity that denotes strong ties (Granovetter, 1973). This result also supports comments made by Matthew that while he and Barbara lead their respective teams, Matthew's role is most senior in relative terms. As such, he commands a stronger individual role within the team, suggesting that tie strength may be linked to hierarchy:

I am the Manager of ... here [Edu1], but there [Edu2] that would probably be the equivalent of two management positions up.

A strong tie also exists between Heather and Peter from Edu2. This tie indicates that in their isolation from other members of the network, these two actors have forged a strong reliance on each other. This may have eventuated from the surprisingly weak tie between Barbara and Matthew. Despite the fact that Peter seeks information from others, the majority of these attempts represent weak ties.

\subsection{Summary of Chapter 4}

This chapter has presented the key results and analysis of Case 1 of this research. The first section presents the findings related to ICT within the Case. The chapter continues with the findings related to SC and specific aspects of relational, cognitive, and structural SC that play an influence within the Case. The chapter also identifies the informal network evident within the Case and presents both visual and quantitative measures to describe the configuration of the network and roles of individuals within the network. The chapter concludes with a summary and conclusions drawn from the main findings from Case 1.

Analysis of the case results indicates that knowledge sharing in Case 1 typically occurs between members of the same organisation rather than across the inter-organisational team. This was reflected in participants' interview comments and through the informal network mapped using SNA. This organisational division characterises all interaction in the collaboration and, in many respects, limits the potential benefits that could be derived from collaboration between members of each organisation. Participants' length of tenure and range of experience in the education field are extensive and provide a valuable network of expertise. However, the informal network shows that this expertise and experience is mostly limited to interaction within the respective teams. 
The exchange of educational data is the key focus of the collaboration, and this dominates the interaction between participants. Knowledge sharing is most likely to occur through face-to-face communication (between members of the same organisation) and via email (across the inter-organisational team). The focus of interactions between participants in less senior roles relates mainly to clarifying aspects of the data exchange, whereas senior level interactions reflect issues related to deriving meaning and understanding from the data, and are more closely aligned with the sharing of knowledge. The findings suggest that information is controlled by the respective team leaders of the two participating organisations and that this inhibits the flow of information to some members of the team, particularly in Edu2.

Although the Case recurs annually and some participants, including the team leaders, have been involved in several instances of the collaboration, there appears to have been little change in the way the collaboration is conducted, despite the issues revealed in this research. The development of a shared terminology and the ability to integrate ICT systems would clearly facilitate improved information and knowledge sharing. The fact that this has not happened suggests that either the collaboration is not deemed sufficiently important to warrant this investment, or that individuals in this collaboration are not significantly positioned within their organisations to instigate change.

The lack of ICT tools available to participants is a surprising outcome of this Case, given the government's historic commitment to ICT through its e-government project, and related initiatives. The Case has no access to shared drives, shared workspaces, or other collaborative tools, and this limits participants' options for communication. However, this is not an issue for participants who expressed a preference for greater face-to-face communication rather than access to a greater range of ICT tools.

The main barriers faced by participants in Case 1 derive from organisational level issues, such as a lack of a common language between the two organisations; differences in the overall purpose and goals of the organisations; and decisions made at a senior management level. The majority of these barriers relate to aspects of SC, which plays a key role in Case 1. The findings suggest there are several opportunities whereby a greater emphasis on SC at an organisational perspective would enhance the interorganisational collaboration. For example, focussing on the development of a shared language across the organisations, and ideally across the sector, would provide a solid 
foundation for the capture, analysis, and sharing of data between the organisations. It would help to alleviate much of the time-related pressure that participants currently face, and would provide more opportunity for participants to derive greater value from the collaboration, rather than focussing on ensuring the correctness of the raw data.

It is to the credit of individuals and their commitment to their work that the issues encountered by participants in this Case do not present a greater obstacle than they do. There is a belief that working within the sector denotes a sense of value through individuals' contribution to the development of the New Zealand education system. This commitment to the education sector (and consequently to the collaboration) underlies motivation for individuals to share knowledge, and provides the basis of the sense of identity that is associated with both the wider work programme and participation within the education sector. However, while these findings indicate that issues of SC are considered important to individuals and to the inter-organisational team, organisational-level issues means that the onus is on individuals to foster SC, and that this is not always supported from individual organisations. 


\section{CASE 2 RESULTS}

This chapter presents Case 2, the second of the four cases explored in this study. The presentation of the findings follows the structure presented in Case 1.

\subsection{Overview of Case 2}

This section provides an overview of structure, purpose and activities of Case 2. The section also documents the number of participating organisations, a breakdown of the members from each organisation, and the phases of the research in which they participated.

Initiated in 1996, this Case is based in the New Zealand Justice Sector. The sector is comprised of six organisations that form the core of the justice sector system (MOJ, 2010). This Case represents a single collaborative venture within a broader programme of work relating to the Justice Sector's information strategy.

This Case involves five organisations and is led by Jus1 who were the instigators of the collaboration and who are responsible for overseeing and co-ordinating it on a day-today basis. Each of the participating organisations operates in locations throughout New Zealand.

The purpose of this Case is to support the sector's information strategy through the development and maintenance of a shared data dictionary. The dictionary enables all organisations participating in the sector to adopt the same terminology. Due to the ongoing nature of this collaboration, the Case has no finite lifetime.

The impetus for the collaboration arose from a move away from a sector-wide shared information system, to an approach where each organisation implements and maintains individual systems, thus risking an inconsistency of terminologies used between systems.

The dictionary defines all the data shared across the common information systems within the sector, but is limited to those parts of the data model that are common to two or more organisations. Therefore, if an individual organisation implements a new 
information system in, for example, the human resources department, they are not obliged to use the standard terms defined within the data dictionary.

Initially, the business of the inter-organisational team was conducted on a relatively informal basis, with no formal structure, shared processes, or recordkeeping. Over time this changed and the collaboration adopted a more formal approach. This includes a formal terms of reference, and standardised processes and procedures that guide the activities of the collaboration. There are also regular meetings that team members can attend, and papers and discussion points are disseminated prior to the meeting. Minutes of meetings are recorded and made available to participants.

No formal reporting structure exists within the inter-organisational team, and the collaboration is structured as a committee with a nominal Chairperson. The Case operates on a consensus basis with co-ordination of the group undertaken by the Chairperson who, for the last three years, has been the most senior of the Jus1 representatives. The need for consensus ensures that each participant has an opportunity to represent their organisation's view on suggested changes to the dictionary. In many cases, this is actioned through a simple agreement to the suggested change. In other instances, where participants do not agree or need to debate the change, a more in-depth interaction with other participants is required and represents a more extensive or comprehensive exchange of opinions. Decisions that have an impact in the wider government environment or on specific groups within the sector are often referred to other committees comprising individuals in more senior roles than those within this team.

A total of ten individuals participate in this Case. Five of the participants are employed in Jus1, the lead organisation in the collaboration, but work in different business units within the organisation. As such, there is no formal reporting line between them. Other organisations are represented by either one or two individuals (see Figure 24). A breakdown of the roles and tenure of Case participants, and the role in specific phases of the research is included in Appendix 8.

Participants in Case 2 are geographically dispersed. Jus1 participants are located across two locations in central Wellington; Jus2 participants are located within the Wellington region; the single Jus3 participant is also located in central Wellington; participants 
from Jus4 and Jus5 are both located outside the Wellington region (see Figure 24). Consequently, due to distance, it is more difficult for participants from Jus4 and Jus5 to meet with other participants.

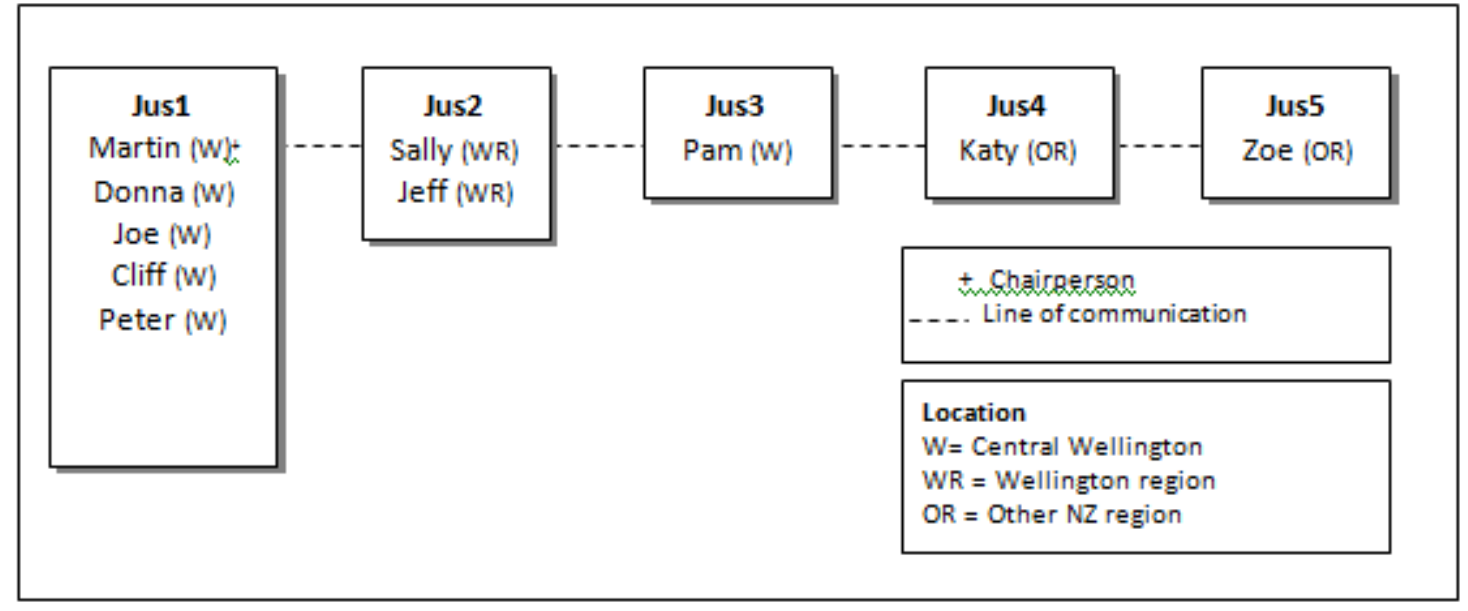

Figure 24: Case 2 Team Structure

Each of the Case participants has an information management or technical background, and holds a related role within their respective organisation. These roles vary but include data architect, applications support manager, and information manager. Despite the difference in roles, there is little difference in the levels of seniority within the group. Participants' average tenure within their individual organisations is approximately $5.6^{10}$ years.

Case activities are conducted as part of the participants' normal day-to-day responsibilities; thus there is no formal resource or funding allocation assigned to the collaboration. Despite the geographic distribution of Case participants, face-to-face meetings are scheduled on a monthly basis, although it is rare for all members of the team to be present. This is particularly the case for participants who are based outside the Wellington region. In this respect, the Case is predominantly conducted as a virtual collaboration, and is categorised as such. The specific characteristics of the Case are noted in Table 31.

\footnotetext{
${ }^{10}$ This figure is based on the tenure of 9 participants who responded to this question, and is skewed by a single participant with a tenure of 19 years.
} 


Table 31: Case 2 characteristics
\begin{tabular}{|l|l|}
\hline Characteristic & Case 1 \\
\hline Physical Type & Virtual \\
\hline Number of Organisations & 5 \\
\hline Number of Individual Participants & 10 \\
\hline Duration of Collaboration & Ongoing \\
\hline Allocation of Staff Resource & Part-time \\
\hline
\end{tabular}

\subsection{Perceptions of Knowledge}

The Phase 2 survey asked participants to define knowledge using their own words. The purpose of this request was to encourage individuals to actively consider knowledge and enable the researcher to identify similarities and differences between individual's perceptions, and to help to determine the value and role that knowledge plays in the Case. A full list of the participants' responses is included at Appendix 8.

The core of this Case is concerned with the way in which information is described and categorised within the Justice Sector. Consequently, participants contend that information rather than knowledge forms the basis of sharing activity within the collaboration, and this is reflected in their consideration of the concept of knowledge. In this respect, most participants use information as the basis for their knowledge definitions.

Information is described as the basis from which knowledge is built or developed, through a combination of its use and application in conjunction with an individual's experience and expertise:

Information, of which someone is aware, has an understanding of and acts on for specific purposes - such as performing their job.

Participants also perceive knowledge as tacit in nature and note that, in some instances, knowledge cannot be written down:

Sometimes things that can't be written down because they are difficult to describe - intuition. 
Participants were familiar with the term "KM", although opinions about the value of the concept are mixed. Some individuals indicate that, in their opinion, KM is the current "buzzword". By contrast, a Jus1 participant observes:

In this building, which is more policy, they totally understand KM and that team has done a very, very good job selling good KM practice.

At an individual level, discussion revealed that there are two very distinct knowledge types in evidence within this collaboration. Firstly, participants exhibit strong technical knowledge. Technical knowledge is related to the extent and depth of participants' understanding of subject domain knowledge, and which is demonstrated through the ease with which team members make decisions to proposed changes to the data dictionary. Secondly, discussion with interview participants reveals the extent of institutional knowledge that individuals have acquired. Both the depth and range of this technical and institutional expertise have been formed through the participants' long tenure within the public sector, in a variety of different organisations. These knowledge types manifest in different ways. Technical expertise is most commonly demonstrated through the discussions that are conducted in relation to proposed changes to the data dictionary. By contrast, when the members convene for a meeting, there is likely to be much deeper debate on issues other than those relevant to this specific collaboration, thus reflecting the breadth and depth of participants' institutional and sector knowledge.

\subsection{Knowledge Sharing Activities}

An initial evaluation of the Case interview data suggests that knowledge sharing in the collaboration is relatively straight-forward. As noted in the previous section, pparticipants demonstrate a shared understanding of knowledge through their use of similar descriptors (see Appendix 9). They also agree that knowledge sharing is actively encouraged across the inter-organisational team (see Appendix 12). However, further analysis reveals several participants are frustrated with the collaboration. This has impacted on individuals' attitudes to knowledge sharing within the venture, and several participants consider that the overall operation of the collaboration could be vastly simplified.

Knowledge sharing activities in this Case are initiated by a proposed change to the data dictionary. This is undertaken using one of several ICT-based tools available to 
participants. Subsequent activities involve the exchange of opinion and discussion relating to the proposed changes, and are undertaken through a combination of face-toface and ICT-facilitated communication. An overview of these activities is depicted in Figure 25.

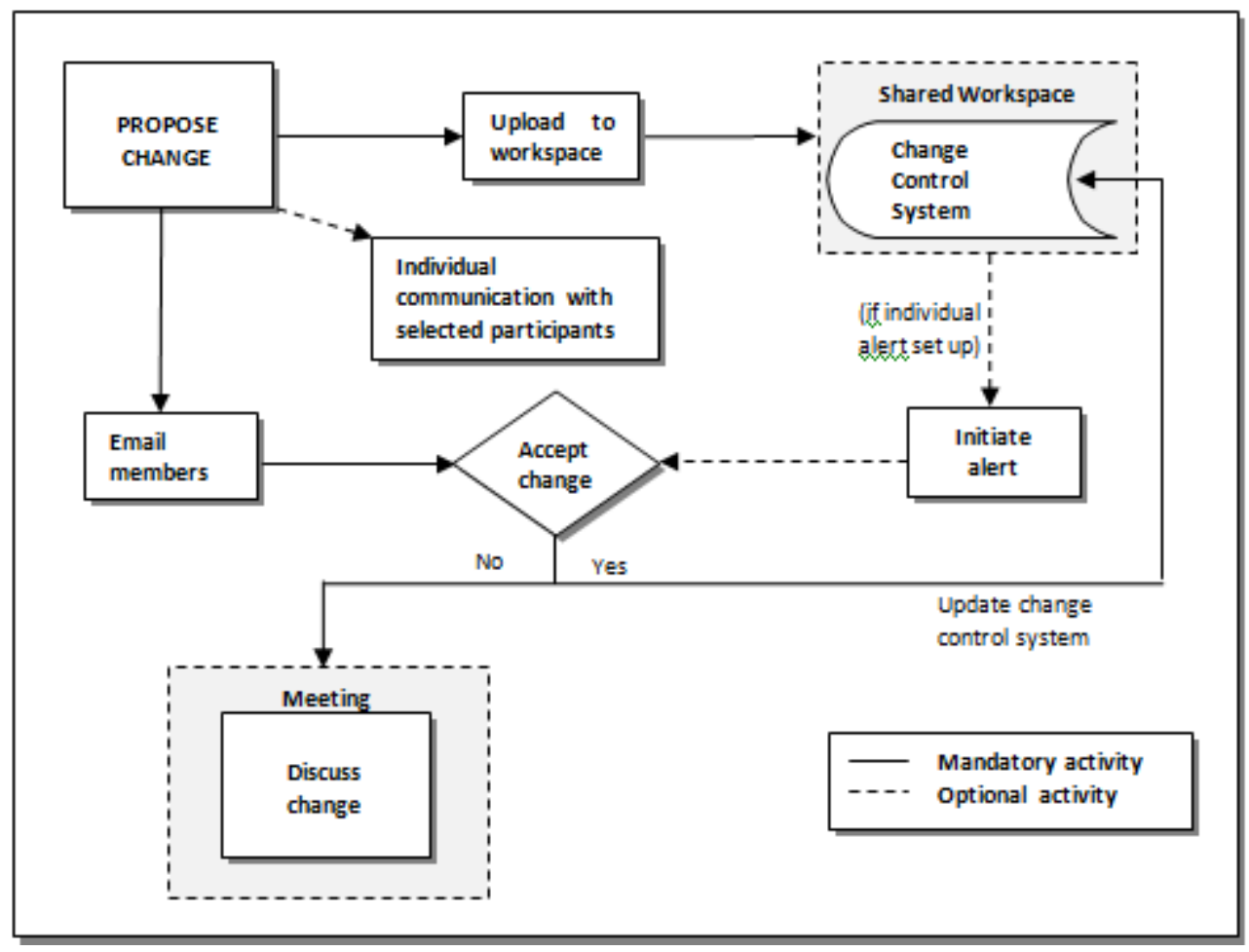

Figure 25: Knowledge Sharing Activities

As Figure 25 shows, a participant uploads a proposed change to the change control system that is located on the shared workspace (discussed further in Section 5.4). The shared workspace has the ability for individuals to be automatically alerted if any new documents are loaded, or changes to the dictionary are proposed. However, the alerts are user-driven so each member is responsible for setting up the individual parameters for alerts to be sent to them. To compensate for any members who have not set up alerts, emails are sent from the initiator of the change to members each time a document or change is posted. If no-one opposes the changes they are deemed accepted and actioned. If the proposed changes are opposed, or generate significant email discussion, they are scheduled for discussion in more detail at the next monthly meeting. In some cases, team members will discuss the proposed change via telephone, email, or face-toface communication with other Case members before posting it on the workspace. One participant comments that this is seen "as a more effective way of getting things done". 
Initial activity most often focuses on sharing information relating to the proposed changes. Tacit knowledge is most likely to be shared when changes are not accepted and there is a need for more in-depth discussion. The majority of tacit knowledge sharing takes place through formal rather than informal activities, specifically through the monthly meetings. These meetings are mostly attended by members in the Wellington $\mathrm{CBD}$ and greater Wellington region. Participants located outside of Wellington sometimes join the meeting via teleconference.

More than two-thirds of participants state that they learn more from an informal chat than a formal meeting (see Appendix 12). However, opportunities for informal interaction are limited due to the geographic dispersion of Case participants which precludes the likelihood of opportunistic meetings. Discussion with Case participants indicates that several participants have different opinions about the frequency of team meetings. Some believe they occur monthly, others consider them to occur on a two monthly basis:

Sometimes we meet every two months, sometimes it's once a month.

We meet monthly.

We've been meeting monthly, but they're over in half an hour, nothing really happens so we now meet two-monthly.

These comments show that participants are unsure about the actual frequency of meetings, and suggest potential issues with information flow within the team. It also suggests that participants do not attend meetings regularly. In fact, most participants had reservations about the value of meetings, and these reservations extended to the value of the collaboration in general:

A lot of people who were members of the group didn't turn up an awful lot and couldn't understand why they were there because it ran so slowly.

We don't actually have to meet for all the changes to be made; that can occur virtually but we do continue to meet monthly just to discuss the changes.

To be honest, nothing comes out of this collaboration. 
Only two participants suggested that there are positive aspects to the meetings, particularly in enabling members to get to know each other better and as a mechanism for individuals to assess others' honesty or credibility:

I think there is value in the group meeting ... because they meet each other and actually what they do is bring their own knowledge and their background to the table.

You get a sense of whether people tell you the whole truth and nothing but the truth, or manufacture something, or whatever.

They build up credibility amongst one another, so they go back and they get an email the next week saying so and so in [Jus2] is questioning this change to the data table. They respect one another; they know who that person is.

In summary, several participants consider that the monthly meetings, and other formal aspects of the venture have "killed the real collaboration", and that the rigidity of the venture serves as a reminder to individuals about their accountabilities within their parent organisations, and leads to more cautious interaction.

\subsubsection{Choice of communication channel for knowledge sharing}

Case 2 participants have access to a range of mechanisms, including face-to-face meetings, email, telephone, and communication tools provided through the shared workspace.

Email is the most commonly used communication channel, and is the preference of the majority of participants (see Table 32). Only two participants are likely to use the telephone for communication purposes. Although no participants indicated that they make use of the collaboration tools available via the shared workspace (email, shared calendar), the interview process did reveal that a small number of users access the workspace from time-to-time. (ICT use is discussed in Section 5.4). 
Table 32: Choice of communication channel

\begin{tabular}{|l|l|l|}
\hline & \multicolumn{1}{|c|}{$\begin{array}{l}\text { Total } \\
\text { Channel }\end{array}$} & $\begin{array}{l}\text { Interactions } \\
\text { Individuals }\end{array}$ \\
\hline Face-to-face meetings & 12 & 4 \\
\hline Telephone & 2 & 2 \\
\hline Email & 17 & 8 \\
\hline Web-based technology(i.e.shared workspace, electronic collaboration tool) & 0 & 0 \\
\hline Other & 0 & 0 \\
\hline
\end{tabular}

In this Case, the predominant influence on a participant's choice of communication channel for knowledge sharing is concerned with the nature of the knowledge. More specifically, different channels are selected based on the strategic or operational nature of the knowledge, or the complexity of the knowledge. Operational level knowledge is easily discussed face-to-face or via the telephone because it will generally be easy to explain or resolve:

The lower level the piece of work is the more inclined you are to just talk to someone about it.

For knowledge that is more strategic in nature, and consequently more complex, it is unlikely that a conversation will suffice:

If it's a really high level piece of work, for example a strategic plan, they are designed to be very, very accessible and pick them up and spend half an hour reading them and get a huge return from them. In actual fact if you phone the person who wrote it they won't talk to you because it's just too risky to try and unpack it all verbally.

Participants indicate that, in general, the better they know an individual, the more likely they will be to communicate with them face-to-face (see Appendix 12); however fifty percent of Case members also indicate that good working relationships can be developed without face-to-face contact (see Appendix 12). Analysis of the relationship between Case participants shows that, in the majority of instances, participants met for the first time on this collaboration, and were therefore unfamiliar with each other. Therefore, while participants indicate a positive association between familiarity with an individual and choice of communication channel, this was not evidenced in this Case 
due to the fact that a priori relationships were not apparent in the inter-organisational team.

\subsection{The use of Information and Communication Technology}

In Case 2, ICT is used both as a storage mechanism or information repository and a communication tool. These activities are facilitated through the use of three main ICT tools that are used to support the Case, including a change control system, shared workspace, and the use of email and telephone for communication purposes.

\section{Change Control System}

The change control system, although referred to by all team members as a database, is an excel spreadsheet ${ }^{11}$. This forms the basis of the data dictionary and is the mechanism by which proposed changes to the dictionary can be initiated. The spreadsheet maintains a list of the agreed terminology between organisations, and is viewed as a good method of facilitating the proposed changes:

It's easy to use...I use it often.

The other system which we use, which is a straight change control system, is just terrific.

One drawback of the change control system is its limited mandate within the participating organisations. Though the system ensures consistency of terminology, these terms are only mandatory in the use of information systems that are common to two or more organisations. It is not compulsory for information systems that are relevant only to individual organisations, such as access databases that may be used within individual business units:

The dictionary only applies to data that is shared between the agencies. So for example if somebody comes into the Ministry of Justice, into the Department of Corrections to build a new human resource information system, then the data dictionary does not apply.

\footnotetext{
${ }^{11}$ There is currently discussion on using Microsoft Access to store the data in a database format.
} 
Overall, the change control system represents a simple tool and does not require users to have a high degree of technical knowledge to use it.

\section{Shared workspace}

All participants have access to a shared workspace that is administered by Jus1. In addition to the change control system, the workspace stores and makes available meeting agendas, minutes, and other documents that are relevant to the Case, as well as a list of contact details for participants in this Case as well as those in the wider work programme.

The impetus to initiate the shared workspace arose from Jus1's past experience in collaborative projects. As Matthew notes:

We used to host information using a crude website that very much gave a sense of the organisation telling the sector what to do....so we used the e-government environment ${ }^{12}$....it sort of seems neutral and independent.

The workspace is part of the e-government environment and this is perceived as removing ownership from any individual organisation, and increasing a collective sense of ownership or involvement in a collaborative endeavour. The majority of participants are aware of initiatives such as the Government Shared Network (GSN) and shared workspaces, and one participant points out that Jus 1 has been an early adopter of the use of shared workspaces:

I would say generally that I think our [Jus1] use of it [shared workspace] is still fairly rudimentary but if you talk to e-government they will say that we are one of the highest sector users of the shared workspace.

However, the experience of individuals in this Case suggests that the use of egovernment does not influence use; participants report limited use of the workspace, due mainly to difficulties interacting with it. For example, because some members have

\footnotetext{
12 The e-government environment relates to the e-government strategy and related initiatives initiated in 2000 (see the literature review in Chapter Three for more details).
} 
not initiated alerts, the individual proposing a change must ensure that each participant receives an email about it. This results in individuals needing to duplicate work:

I put it up on the shared workspace but then I still have an accountability to ensure our own recordkeeping requirements, so I then post it within our internal document management system. So I do it twice, it's an extra piece of work.

It's user driven so what we still do is post the material up [on the workspace] but we're still sending the emails to say we've just posted up the agenda, because we can't be certain that every person has set up an alert.

Participants reveal other reservations about the workspace:

I find the shared work space difficult. I always forget my password and it's just a really busy page and you've got to find your own group and then you've got to stick in the right thing and if you don't put in the right thing then you're off somewhere else.

I personally don't use it very often. It's not easy.

A further difficulty arises from the maintenance of the Case contact list on the workspace. While changes to the group (replacement or addition of members) are noted on the workspace, those who do not use it will not be alerted to these changes. This is a concern for some members who are unsure whether some individuals were still members or had left the collaboration, and who they had been replaced by.

These findings indicate that even when a workspace is available in a collaboration, this does not necessarily lead to individuals using it, or using it consistently. In this Case, several participants choose not to use it for any tasks other than to access the change control system, citing difficulties relating to access and page layout. So, the success of workspaces cannot be measured simply by the number of workspaces that have been implemented; rather analysis of actual use of the workspace must be undertaken to determine if the workspace is regularly and consistently used. 


\section{Email and Telephone Communication}

Finally, ICT plays a role in facilitating communication between participants. As shown in Table 32 above, email is the most frequently used channel, and sixty percent of participants consider that email communications can help build trust between individuals (see Appendix 12). However, as indicated above, some participants believe that email is most effective once a face-to-face relationship has been formed:

They go back [after the meeting] and they get an email the next week saying so and so in [Jus2] is questioning this change to the data table. They respect one another, they know who that person is.

Email is used for two main purposes: firstly as a tool to notify participants of proposed changes to the data dictionary; and, secondly, as a general tool for discussion about aspects of the Case. There are some difficulties associated with the use of email as a notification system. For example, participants who have signed up to email alerts via the shared workspace, receive duplicated notifications alerting them to proposed changes. Participants must also ensure that they maintain their organisational email contact list to reflect any changes to membership of the Case. These changes are maintained through the workspace but, where automatic alerts are not in place, these changes are not routinely updated to members.

Participants also use email to discuss changes that may be more contentious than others. Though the monthly meetings were developed to enable this debate, participants are more likely to action this through an electronic communication channel. Most participants prefer email to the telephone, especially if the choice is between leaving a voice message and sending an email:

Sometimes I will try and ring someone but if they're not there then I'll put it in an email because I may forget to ring them back or giving them a message when they ring you back and you're not there and you can play phone tag for three days.

This suggests that email communication benefits individuals by enabling them take action on a particular task and move on to other activities and is, therefore, viewed as more efficient than other channels such as the telephone. 
Overall, participants agree that ICT makes it easier to share information and confirm that within their parent organisations there is a strong emphasis on using ICT to share. Eighty percent of participants think that the ICT tools available to them in this Case are effective.

\subsection{Social capital}

This section explores SC and how it influences knowledge sharing in Case 2.

\subsubsection{Relational social capital}

Findings relating to relational SC were derived from the interviews conducted with Case 2 participants and survey responses. During the interview process, some participants appeared reluctant or unsure about how to discuss some aspects relating to relational SC. The basis of this reluctance and uncertainty appear to be related to two considerations: firstly, whether their comments would identify them in any way; and, secondly, a perception that the collaboration does not necessarily require relational SC to function and that, and comprise an additional overhead (additional demands on the individual's time) that is not required.

\section{Identity}

The majority of Case 2 participants do not consider that the inter-organisational team demonstrates any sense of group identity. Indeed, one participant believes that the ability to generate a sense of group identity in public sector inter-organisational projects per se is negatively impacted by the sector's approach to collaboration:

The accountabilities are very much on agencies, so when you get a collection of organisations together, although there may well be a mandated leadership role, [individual's] accountabilities are to the agencies.

This places members of inter-organisational teams in the position of needing to ensure that any information or knowledge that is shared with other team members is in alignment with internal organisational policy. They must feel confident that use of the information by other participants or their parent organisations will not place them or their organisation at any risk. 
The absence of a sense of a group identity stems from three main factors: the nature of individual's involvement in the collaboration; concerns about the purpose of the collaboration and the way in which it is undertaken; and allegiance of the participants to their parent organisations.

Participants have joined the collaboration at various stages of its development, and their involvement is driven solely by their role within their parent organisation. So, as people change roles the membership of the group also changes.

People tend to join this group and stay as long as they're in the sector; one of the things that was hard to get going with the group was that people tended not to want to join.

Participation, then, is regarded as an obligatory duty rather than a voluntary commitment. This has impacts on both the individual in terms of their commitment, and the ability of the inter-organisational team to develop a sense of connection between members.

This is compounded by the fact that there is no formal introduction to the interorganisational team, other than a change to the contact list maintained on the shared workspace. It is feasible that if a participant agrees with all proposed changes, and does not attend the team meetings, they may have no interaction with other team members. Such instances are likely to further impact the team's lack of identity.

Only Martin indicates a clear sense of alignment, or identity, with the interorganisational team. He states:

It [data dictionary] is widely regarded as being a very significant piece of work....So people have a real sense of value of it. People have got a desire to be around the table and to be associated with it.

Martin's involvement with the collaboration differs quite significantly from that of the other Case participants. He was heavily involved with the inception of the data dictionary, at a more strategic level, and feels a strong sense of commitment and identity with the maintenance of it. This suggests that involvement at a strategic level, or from 
the outset of the development of a collaborative endeavour, may increase an individual's sense of identity with a collaboration.

Identity is also adversely affected by individuals' frustrations about the collaboration, particularly in relation to the processes and activities. As a result, participants perceive some of the activities of the collaboration, particularly attendance at meetings, as unnecessary interruptions to their working day, and this impacts on the sense of connection that individuals feel toward the group.

Discussion relating to identity indicates that participants are most closely aligned to the identity of their parent organisation, and that this can impact both their ability and willingness to share knowledge with other Case participants. Several individuals note that sharing information can sometimes expose both individuals and their organisations to risk:

If I share information with a colleague, then it's my accountability and I am answerable within my organisation - not to any of the other agencies.

This appears to reinforce the participants' concern about protecting both themselves and the organisation. This manifests through interaction with other participants. In some instances, interaction is driven more by individuals' concern about "being seen to do the right thing", than by a sense of identity or commitment to the inter-organisational team.

\section{Trust}

Case 2 participants consider trust an important aspect of collaboration. Despite this, participants perceive actual trust levels in this Case to be average or below average (see Appendix 12).

Analysis identifies several factors that contribute to trust levels in this Case including a lack of interaction between team members and the potential for conflict arising from organisational accountabilities.

Survey responses indicate that while forty percent of participants base their decision to trust a team member on the competency level of the individual, twenty percent believe that trust is based on the relationship with other participants and how well they know them. This suggests that eighty percent of participants build trust through some kind of 
interaction, whether that is based on getting to know an individual, or through an interaction that enables competence to be demonstrated. This is an issue for this collaboration, in which, theoretically, members can participate without interacting with others. In these instances, individuals lack the foundation elements on which a trust relationship can be built. Therefore this lack of overall interaction may contribute to participants' perception of less than optimum trust levels within the Case.

The type of interaction does not appear to influence trust levels. Although several participants indicate that, in their experience, trust is strengthened through face-to-face communication, survey responses indicate that fifty percent of participants consider that good working relationships can be established without face-to-face communication. This finding is reflected in participants' lack of support for team meetings, which are not considered conducive to building trust:

The first three times I turned up I just couldn't figure out why it was so slow, why people weren't simply doing the job and I then realised that it was really a trust issue.

I mean there are people around who believe that information is power and knowledge is power and so on, but I haven't got any time for them.

However, one participant believes the meetings provide a good opportunity to build trust by getting to know other people and become familiar with their experience and expertise.

They [the meetings] can help people get to know each...they are an opportunity to build relationships.

Further, this building of trust can change the way that individuals respond to ensuing electronic communication that they receive from others:

They've got an understanding that that person actually has got twenty years experience... so [they] are not going to log a frivolous or unnecessary request.

Issues related to organisational accountability (as detailed in the previous section), also impact knowledge sharing in inter-organisational projects. Participants note the 
potential risk arising from sharing information and knowledge with other team members, and believe that this inhibits the development of trust in the team:

The risk [of information sharing] all resides with the individuals in the agency.

Therefore, without a clear mandate to share from their respective organisations, it is likely that individuals will be less inclined to demonstrate open sharing behaviours.

\section{Norms, Obligations and Expectations}

Group norms represent an agreement or consensus among participants about the way in which the group operates. In this Case, factors relating to norms, obligations, and expectations act to both support and inhibit knowledge sharing among members of the inter-organisational team.

The formal nature of the collaboration, and the way in which documented processes and procedures are in place to guide participants, mean that there has been little opportunity for Case participants to establish their own set of norms. This is ironic given that this level of formality was introduced to aid collaboration but has, in most participants' opinions, effectively inhibited a natural sense of collaboration that could be built through the opportunity for the group to develop its own norms.

The obligatory nature of individuals' participation in the Case also affects knowledge sharing within the collaboration. Case members are obliged to participate in the collaboration as a responsibility stemming from their role within their parent organisation. While this in itself is not an issue, concern about the value and purpose of the collaboration has led to some resentment regarding participation, and some members indicate that if it were not for their obligation to contribute, they would be unlikely to do so. This is also evident from the analysis of individuals' motivation to share knowledge which shows only three of the ten participants are motivated to share through their commitment to the collaboration (see Table 33). It is likely that this sense of obligation also negatively impacts participants' identification with the Case, and thus inhibits the development of a sense of group identity. 


\begin{tabular}{l} 
Table 33: Motivation to Share Knowledge \\
\begin{tabular}{|l|c|c|}
\hline Motivation & $\begin{array}{l}\text { Total } \\
\text { Count }\end{array}$ & $\begin{array}{l}\text { No of } \\
\text { Individuals }\end{array}$ \\
\hline Trust in the individual & 2 & 2 \\
\hline The feeling I am able to help that person & 10 & 2 \\
\hline The belief that the recipient will share their knowledge with me in return & 5 & 3 \\
\hline Commitment to the collaboration & 10 & 3 \\
\hline Presence of technology that makes sharing easy & 0 & \\
\hline Recognition of my own knowledge and expertise & 0 & \\
\hline
\end{tabular} \\
\hline
\end{tabular}

During the interview process, two participants suggested that the monthly meetings provide an opportunity for individuals to build recognition and respect amongst team members, through the demonstration of their experience and expertise. This suggests that the expectation of respect or reputation building could facilitate knowledge sharing between participants; however this was not found to be an important issue for the majority of Case participants who appear more concerned with the functional aspects of the collaboration than potential enhancement to their professional reputation.

\subsubsection{Cognitive social capital}

Cognitive SC focuses on issues of shared language, shared purpose and goals, and a sense of shared culture.

\section{Shared language}

The development and maintenance of a shared language across the sector is the primary purpose of this Case. In this respect, participants are clear about the importance of shared language and believe that it provides an important asset for the sector; an asset that supports sector-wide communication enables individuals to use terminology that is consistent regardless of what organisation an individual belongs to.

However, despite best intentions, the existence of the data dictionary does not ensure that terminology is consistently applied in all instances:

There will also be examples when it [data dictionary] is not used...you just can't control everything.

Evidence of this can be seen at an organisational level, where mandated use of the data dictionary applies only to ICT systems that hold information that is shared across the sector, that is, between two or more organisations. This means that information systems 
developed solely for internal organisational use are not formally bound to use terminology consistent with the data dictionary. While this may not be important for systems that hold information relating to employees (i.e. Human Resource Information Systems), or finance (e.g. Financial Management Information Systems), it is inevitable that other systems will be developed in-house that, while not formally intended for use across the sector, will contain information that relates to the subject domain. For example, development of Microsoft Access databases to store subject matter information within teams is common in organisations. Not applying the mandated terms set out in the data dictionary is likely to cause confusion and information redundancy.

\section{Shared Purpose and Goals}

The early stages of data gathering (Phase 1 interviews) indicated that the purpose of the Case was clearly set out in the documentation relating to the collaboration, and that this is intended to create a sense of shared purpose among participants. This is not the case. Participants report that they and their organisations are supportive of a shared data dictionary for the sector, and to this extent participants understand the project's purpose. However participants' actual experiences have led them to question the the way in which the collaboration seeks to achieve this purpose and the value of the project. Some participants believe the venture is over-engineered and could be significantly simplified:

I couldn't understand why we were making it so hard... the reason that people are there is to give the process legitimacy.

It's very hard because I'm never really sure what the purpose of the working party is. I mean I know theoretically what it is, but I've been involved 18 months now and to be honest nothing's come of this ... particular group.

These concerns are also reflected in participants' low level of commitment to the collaboration (see Table 33 above), which is, in turn, associated with trust levels and the level of interaction between participants.

In addition to the issues outlined above, participation in the collaboration must be incorporated into everyday activities and, at times, it is difficult to allocate time within 
busy workloads, particularly to attend monthly meetings. Several participants believe that the collaboration could occur without such a high level of formalisation, and without any need for meetings. This opinion is endorsed by survey responses which indicate that Case participants believe good working relationships can be established without face-to-face contact. This suggests that there may be scope to redefine how the collaboration operates, and that this could ultimately lead to a better sense of shared purpose and higher levels of commitment toward the venture.

\section{Shared Culture}

Participants observe that the culture of the justice sector is one that operates conservatively, and has a regard for hierarchy:

The Justice sector generally is a very conservative group of agencies which you know you might expect. Hierarchical.

However, some participants note that sub-cultures are evident within the overall justice sector, and that this can bring diversity to inter-organisational projects, even those that contain a majority of organisations from a single sub-sector:

Even within that general framework [the Justice Sector] there are very strong cultures. So each person brings quite a, a different feel to a working group.

Participants who are not Jus1 members believe that Jus1 is characterised by a level of bureaucracy and process that is less evident within their own organisations:

We [Jus 3] don't have so many levels of authority.

They [Jus1] are very cautious...there's a lot of bureaucracy.

This is supported by survey responses which show that sixty percent of the team consider that the culture of the inter-organisational team differs from the culture within their own organisation (see Appendix 12). Further, survey responses show that fifty percent of Case participants also believe that a small subset of the inter-organisational team determines the dynamics of this inter-organisational team (see Appendix 12). Analysis shows that the majority of these responses are attributable to non Jus1 members. This implies that Jus1 members have a higher level of influence on the way 
in which the collaboration operates. This finding reflects the role of the Jus 1 within the collaboration, holding the informal leadership mandate and comprising half the interorganisational team.

One participant suggests that a mechanism for dealing with culture differences is to be aware of what issues other organisations may be facing, and how this could be affecting the current cultural climate within their organisation. This can help to ensure that participants understand the pressures that other individuals in the team may be facing:

We [Jus1] try to be very, very conscious about issues that are affecting the organisations that we are dealing with. I mean it's very hard to be really aux fait with culture if you're not part of it; but we do make very strong efforts to be at least aware of what current issues are being faced in an organisation.

Although notionally a positive approach, it does not appear to succeed in practice. Jus1 members, as the majority group and the informal lead organisation, clearly have a higher level of influence on the way in which the collaboration operates. This may lead other participants to feel disenfranchised, lead to lower levels of commitment from non Jus1 participants, and influence the levels of trust between team members.

\subsubsection{Structural social capital}

Structural SC focuses on the formal structure (who reports to whom) and the informal structure (who interacts with whom). The formal structure detailed in Section 5.1, identified that the collaboration operates as a committee where decisions are made by consensus, and is guided by a nominal Chairperson. The collaboration is also guided by a formal terms of reference, and are documented processes and procedures that guide how work will be carried out.

Figure 26 presents the informal network depicted in the visual network of Case 2 . The results show that while interaction does occur between Case participants, the frequency of interaction is limited, and most interactions occur less than once a week. The results also show the presence of eight reciprocal relationships, where participants both seek information from, and provide information to, each other. There is only 1 clique evident with the network (Joe, Cliff, and Martin) indicating that, in general, information flows through the network based on need rather than because some actors are more 
intensely linked. However, Martin provides the single cutpoint in the network; he provides the only connection between Peter and the rest of the network.

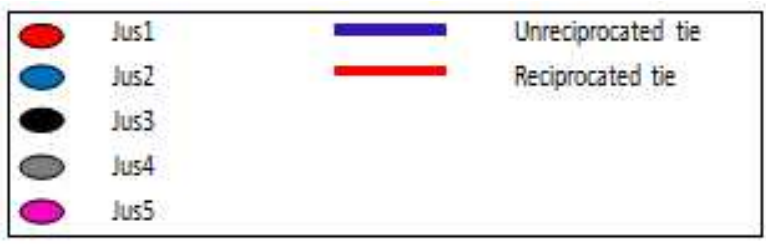

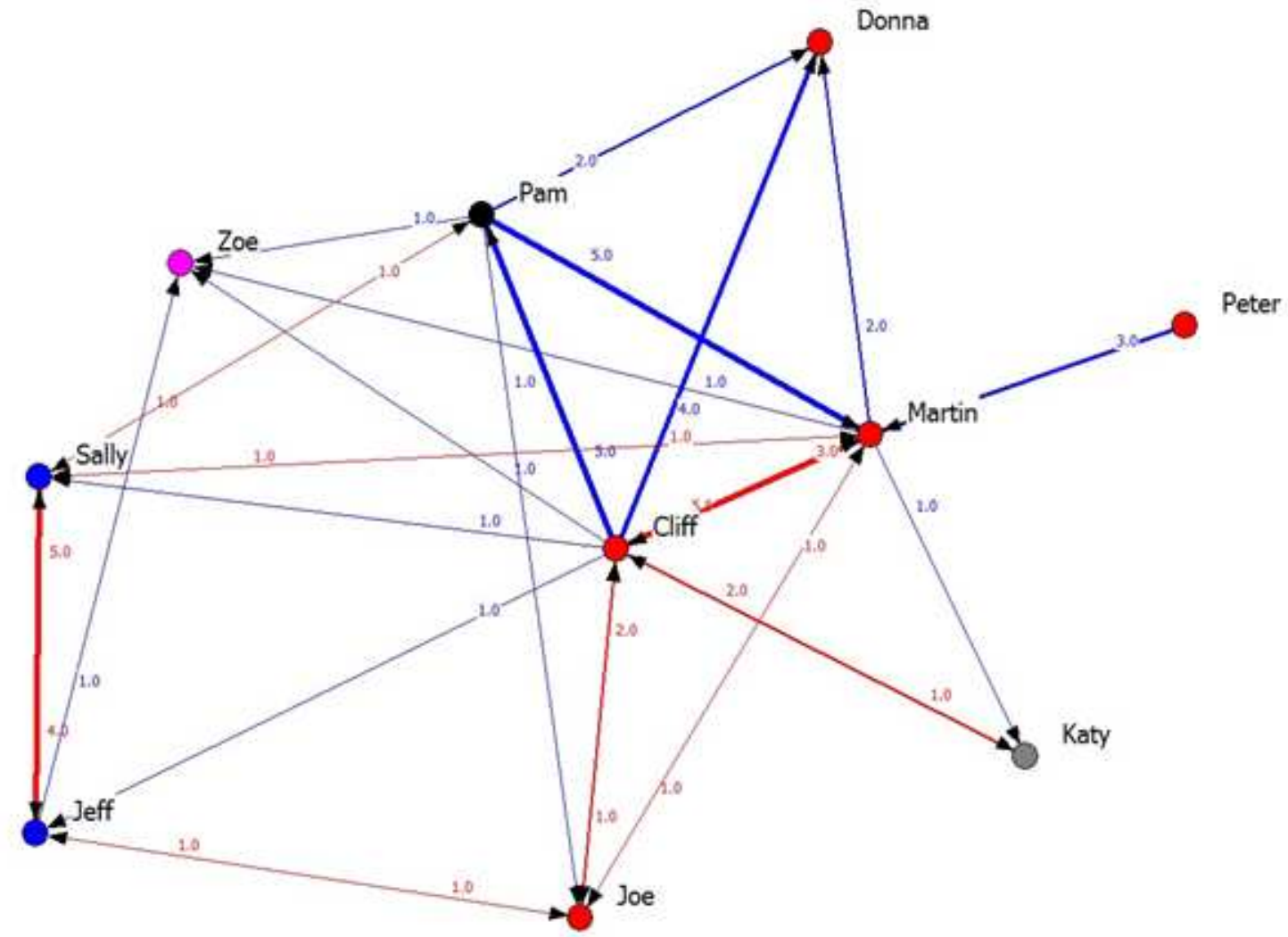

Figure 26: Case 2 Informal Network

Analysis of the network identifies findings at both the network and individual levels, and these are presented in the following sections.

\subsubsection{Network level results}

Table 34 provides an indication of the health and overall cohesion of the network. (Extended descriptions of these measures can be found in Chapter 3, Section 3.3.4.2.) 
Table 34: Case 2 network level measures

\begin{tabular}{|l|l|}
\hline Measure & Result \\
\hline Size & 10 \\
\hline Total Ties Present & 29.0000 \\
\hline Density (Mean) & 0.3222 \\
\hline Distance & 1.692 \\
\hline Std Deviation & 0.467 \\
\hline Variance & 0.218 \\
\hline
\end{tabular}

The network size of 10 indicates the number of participants within the interorganisational team, and identifies as the smallest network in this study. In networks of this size, it is easier for individuals to get to know others than in networks that include a greater number of participants. The network density, or overall connectedness, for Case 2 is $32 \%$. This means that approximately one-third, or a total of 29 ties, of the total possible ties (100) are in place across the network. Given that there are only 10 participants in the network, and that five of these participants belong to the same organisation, this density is lower than would be expected for a network of this size. The density level supports the earlier finding that, in this Case, it is totally feasible for individuals to have no direct interaction with other participants. For example, if an individual accepts all proposed changes then they can do so simply by indicating acceptance through the change control system, eliminating any need for interaction with other team members.

The average distance between individuals, or the degree of separation, is 1.692. This suggests that should individuals require information or assistance that is not available from actors to whom they are directly connected, they can generally find this information with relative ease. So, despite the low level of cohesion across the network, individuals are able to quickly access collaboration-related help and advice when needed.

\subsubsection{Individual level results}

As in Case 1 the three measures - degree, closeness, and betweenness centrality were applied to Case 2. (Fuller descriptions of these measures can be found in Chapter 3, Section 3.3.4.2.) 


\section{Degree Centrality}

A full list of normalised degree centrality degree ${ }^{13}$ scores for Case 2 is shown in Table 35. These measures show a large variance between the highest (88.889) and lowest (11.111) scores of actors, and indicate that some individuals are likely to find it more difficult to source information within the Case. It is interesting to note that both the highest and lowest scoring members of the inter-organisational team (Martin and Peter) are members of Jus1, but work in different locations. This suggests that co-location is more influential than organisational identity in terms of network ties.

Table 35: Case 2 degree centrality measures

\begin{tabular}{|c|c|c|c|c|c|c|}
\hline $\begin{array}{l}\text { Degree } \\
\text { Centrality }\end{array}$ & Actor & $\begin{array}{l}\text { Formal } \\
\text { Role }\end{array}$ & Organisation & NrmOutdegree & NrmIndegree & NrmDegree \\
\hline \multirow[t]{9}{*}{+} & Cliff & Member & Jus1 & 88.889 & 33.333 & 88.889 \\
\hline & Martin & Chairperson & Jus1 & 66.667 & 55.556 & 88.889 \\
\hline & Pam & Member & Jus3 & 55.556 & 11.111 & 66.667 \\
\hline & Jeff & Member & Jus2 & 33.333 & 33.333 & 44.444 \\
\hline & Joe & Member & Jus1 & 33.333 & 44.444 & 44.444 \\
\hline & Sally & Member & Jus1 & 22.222 & 44.444 & 44.444 \\
\hline & Zoe & Member & Jus5 & 0 & 44.444 & 44.444 \\
\hline & Donna & Member & Jus2 & 0 & 33.333 & 33.333 \\
\hline & Katy & Member & Jus 4 & 11.111 & 22.222 & 22.222 \\
\hline- & Peter & Member & Jus1 & 11.111 & 0 & 11.111 \\
\hline
\end{tabular}

Martin and Peter's positions in the informal network also reflect the level of commitment and enthusiasm that these participants indicate toward the collaboration during the interviews. While Martin is very positive, Peter indicates a high level of frustration about the collaboration and its workings. These findings suggest that individuals' attitudes towards aspects of relational SC may influence their interaction with other group members and affect their role in the informal network.

To derive greater certainty and clarity about degree centrality, measures were analysed further to determine the measure of in-degrees and out-degrees. In-degrees denote the total number of incoming connections, or information seeking requests received by an individual. Out-degrees denote the number of the number of information seeking requests that the individual makes of others.

\footnotetext{
${ }^{13}$ Normalised scores divide simple degree by the maximum degree possible and enable cross case analysis to be undertaken on networks of different sizes.
} 
There was very little variance (2.09) between the in-degree scores of individuals. This is representative of the qualitative data findings that note the equal status of individuals and the evenly balanced nature of their skills and expertise. It suggests that interactions are needs-based. For example, changes may be proposed by any actor, therefore queries and discussion regarding a particular change are most likely to be directed at the individual proposing it. As such, information seeking was evenly distributed within the network. One obvious exception to this finding relates to Peter, from whom no participant sought information or advice. This provides support for the earlier supposition that, where participants demonstrate lower levels of relational SC, their interaction with other Case participants may be limited or inhibited.

There was a higher variance between out-degree scores (6.49) indicating that some individuals seek information more often than others. In Case 2, Martin, Cliff, and Pam's out-degree scores are significantly higher than most other members. Out-degree scores are often indicative of an individual's influence within a network; analysis of these out-degree scores, and consideration of comments about these participants, suggests they are highly respected within the collaboration. It should be noted that both Donna and Zoe indicate that they do not seek information from others within the collaboration; it is likely that these members are generally accepting of most proposed changes and do not therefore need to seek information or assistance from others.

Degree centrality was also used to measure network centralisation. Network centralisation measures the global or macro level centralisation of the network and is indicative of how unequal the distribution of centrality is in it. For example, high degree centralisation scores indicate that networks are dominated by one or a few central actors. Case 2 network centralisation measures are shown in Table 36.

Table 36: Case 2 network centralisation index
\begin{tabular}{|l|l|}
\hline Centralisation Index & Result \\
\hline In-degree & $25.93 \%$ \\
\hline Out-degree & $62.96 \%$ \\
\hline Total degree centralisation & $50.00 \%$ \\
\hline
\end{tabular}

The overall degree centralisation index $(50.00 \%)$ indicates that although no single actor plays an overall controlling role within the network, the network shows some reliance on a small subset of actors. This is likely to reflect the fact that some participants (e.g. Martin and Cliff) reflect a higher degree of identity with the inter-organisational team 
and a greater sense of commitment to the work of the inter-organisational team than others. Thus, they interact more frequently within the network and play more influential roles. Overall, the degree centralisation score is representative of a network of this type that operates as a committee and operates on a consensus basis. It denotes the equal status and the high degree of equality between Case participants.

\section{Closeness centrality}

A full list of the actors' closeness centrality measures are shown in Table 37. As evidenced in the distribution of degree scores, there is relatively little variance between the closeness scores of actors. Although both Martin and Cliff are situated in closest proximity to other actors, the overall measures suggest that the majority of actors occupy favourable positions within the network.

Table 37: Case 2 closeness centrality measures

\begin{tabular}{|l|l|l|l|l|}
\hline $\begin{array}{l}\text { Closeness } \\
\text { Centrality }\end{array}$ & Actor & $\begin{array}{l}\text { Formal } \\
\text { Role }\end{array}$ & Organisation & $\begin{array}{l}\text { Total } \\
\text { Closeness }\end{array}$ \\
\hline+ & Martin & Chairperson & Jus1 & 74.032 \\
\cline { 2 - 5 } & Cliff & Member & Jus1 & 74.032 \\
\cline { 2 - 5 } & Pam & Member & Jus3 & 68.684 \\
\cline { 2 - 5 } & Joe & Member & Jus1 & 67.5 \\
\cline { 2 - 5 } & Sally & Member & Jus2 & 67.255 \\
\cline { 2 - 5 } & Jeff & Member & Jus2 & 61.458 \\
\cline { 2 - 5 } & Katy & Member & Jus4 & 61.458 \\
\cline { 2 - 5 } & Peter & Member & Jus1 & 57.368 \\
\cline { 2 - 5 } & Donna & Member & Jus1 & 50.909 \\
\cline { 2 - 5 } & Zoe & Member & Jus5 & 50.909 \\
\hline
\end{tabular}

\section{Betweenness centrality}

The results shown in Table 38 identify that Martin holds the most strategic position in the network, and is able to exert a degree of control over the information that flows through the network. Although Martin refers to his nominal leadership role during the interview process, he confirms that this is not the way in which the collaboration operates and that it is his belief that consensus based decision-making is a more useful approach to collaboration and sharing:

We have a formal mandate but we don't lean on that mandate very much. We prefer and we believe that it achieves a better level of buy-in to lead through consensus agreement. 
Five members of inter-organisational team report zero betweenness scores. This indicates that their positions in the network have no strategic advantage and it is unlikely that other actors depend on these actors to make strategic connections for them.

Table 38: Case 2 betweenness centrality measures

\begin{tabular}{|l|l|l|l|r|}
\hline $\begin{array}{l}\text { Betweenness } \\
\text { Centrality }\end{array}$ & Actor & $\begin{array}{l}\text { Formal } \\
\text { Role }\end{array}$ & Organisation & nBetweenness \\
\hline+ & Martin & Chairperson & Jus1 & 24.306 \\
\cline { 2 - 5 } & Cliff & Member & Jus1 & 20.833 \\
\cline { 2 - 5 } & Joe & Member & Jus1 & 7.639 \\
\cline { 2 - 5 } & Sally & Member & Jus2 & 3.241 \\
\cline { 2 - 5 } & Jeff & Member & Jus2 & 2.315 \\
\cline { 2 - 5 } & Donna & Member & Jus1 & 0 \\
\cline { 2 - 5 } & Katy & Member & Jus4 & 0 \\
\cline { 2 - 5 } & Pam & Member & Jus5 & 0 \\
\cline { 2 - 5 } & Peter & Member & Jus1 & 0 \\
\cline { 2 - 5 } & Zoe & Member & Jus3 & \\
\hline
\end{tabular}

The centrality measures detailed above indicate that Martin, Cliff, and Pam hold more central roles in the network than other case participants. To a large extent these positional advantages can be explained by the roles that individuals play, or have played, within the network. Martin acts as the nominal chairperson of the group and is responsible for its overall co-ordination; it is to be expected that he is positioned in a central position within the network. Cliff also plays a co-ordination role and holds responsibility for the technical co-ordination of the change control system, and thus is likely to have a somewhat higher degree of interaction with other actors. Finally, prior to Martin taking up the Chairperson role, Pam was the nominal chair of the group and, as such, is seen as an experienced member who is still influential within the network. So, despite the potential positional advantages of these actors, deeper understanding of their roles, together with the level of variance between actors supports the conclusion that the informal network is largely reflective of the formal team structure that operates as a consensus-based committee. Finally, the centrality measures indicate that both members of the team who are outside the Wellington region (the location of the majority of actors), are within the lowest four scores in each centrality measure. It is therefore surmised that physical proximity of actors influences their role within the informal network. 


\section{Cliques}

One clique, comprising Martin, Joe, and Cliff, is evident within the network. Cliques represent subsets of the network in which actors are more intensely linked (Hanneman \& Riddle, 2005). These three actors all belong to Jus 1 and occupy roles within the organisation whereby it is likely that they will come into contact for matters other than just the inter-organisational team.

\section{Tie Strength}

In general, the network is characterised by weak ties; interactions that are infrequent (less than once a week), and non-reciprocal. This result suggests that, for the most part, interactions are informational-based and relate to a particular change that is proposed; thus unless further debate is required, they are likely to be discrete events rather than an ongoing sharing of information. It also suggest that participants do not regard this collaboration as one that holds long term benefits for them as individuals, and so place limited emphasis on investing in SC and the building of enduring relationships with other team members. The overall measure of reciprocity for the network is $31.82 \%$; approximately one-third of individuals enjoy a mutual exchange of information and knowledge.

The sharing of complex or tacit knowledge is more likely to be undertaken where ties are strong, and the nature of the tie is reciprocal. The two strongest ties extend between Sally and Jeff, and Cliff and Martin. In both instances, actors come from the same organisations (Jus2 and Jus1 respectively); suggesting that tie strength may be related to organisational membership, although this does not hold true for all Jus1 members.

\subsection{Summary of Chapter 5}

This chapter has presented the key results and analysis of Case 2. The first section presents the findings related to ICT within the Case. The chapter continues by presenting the findings related to SC and specific aspects of relational, cognitive, and structural SC that have an influence within the Case. The chapter also presents the informal network evident within the Case, and both visual and quantitative measures to describe the configuration of the network and roles of individuals within the network. 
This inter-organisational collaboration arose from a decision within the sector to move from a single shared information system, to individual information systems within each of the sector organisations. This change exposed the sector to the risk of inconsistent terminology being used by individual organisations. To mitigate this risk, the collaboration was initiated to standardise language across the sector and ensure that data remained consistent across each organisation and could be easily shared between them.

ICT tools available to participants are generally considered effective, although availability does not necessarily lead to use as shown by participants' perceptions and use of the shared workspace. The results show that knowledge sharing is most often conducted through email, and is likely to occur less than once a week. Other than the monthly meetings, there is little ad hoc interaction between group members and face-toface communication is not considered to be an important element of this collaboration. In some instances, participants operate within the collaboration without direct interaction with other members. This is reflected in various participants' interview comments and through the informal network mapped using SNA.

The findings show that SC within the group is not a key consideration of Case members, and this is particularly apparent in the findings relating to both relational and cognitive SC. In general, there appears to be confusion about the purpose of the group and the way in which it operates. Some team members believe trust is the key issue, while others consider that the formality of the venture has "killed the real collaboration", and that the rigidity of the venture serves as a reminder to individuals about their accountabilities within their parent organisations which leads to often cautious interaction. 


\section{CASE 3 RESULTS}

This chapter presents the third case in the study. The results are presented using the same format as for Cases 1 and 2.

\subsection{Overview of Case 3}

This section provides an overview of the structure, purpose and activities of Case 3. The section also documents the number of participating organisations, a breakdown of the members from each organisation, and the phases of the research in which they participated.

Case 3 is based in the New Zealand justice sector. The sector is comprised of six organisations that form the core of the justice sector system (MOJ, 2010). This core sector group also fosters linkages with other organisations who are not part of the core justice sector but who have a shared interest in the sector.

This Case involves four of the core justice sector organisations, three organisations from the wider sector, and two independent external advisors from the justice field. The collaboration was initiated, and is led, by Justice1. It commenced in 2005, and takes place over a six month period ${ }^{14}$.

The collaboration is concerned with a critical criminal justice issue in New Zealand. Current approaches to the issue are not considered to be effective, and the purpose of the collaboration is to make recommendations about alternative approaches to address the problem. The collaboration is considered to be very high profile: the team leader reports directly to the Prime Minister who is particularly interested in the subject area. The nature of the issue is such that it receives high media attention, and it is often the focus of negative publicity.

The key driver for the collaboration, and the reason for the tight timeframe, is the government's intention to make decisions about the issue at a pre-designated time. In

\footnotetext{
14 To ensure consistency across the case studies, Case 3 is reported in the present tense, despite the fact that, unlike the other Cases in the study, it is not an ongoing project and has been completed.
} 
order to make those decisions, the government has asked for a range of alternative approaches to be researched and developed, and has stipulated that this must be done within the current timeframe. In other circumstances, work of this nature would probably be undertaken over a 2-3 year period, thus this adds to the pressure placed on the inter-organisational team.

The team comprises fourteen members. Eight of the members represent Justice1, the lead organisation in the Case; three members are employed within other core justice sector organisations (Justice2, Justice3, and Justice 4); one team member represents an organisation from the wider public sector; and the remaining two participants are external advisors who provide independent advice to the team. Figure 27 depicts the structure of the inter-organisational team.

All members of the team, except the independent external advisors, are co-located in a single, centrally located, office. The office is not housed within the lead organisation's building as is often the case with inter-organisational teams, and as such, is seen to be independent of any particular organisation.

Rebecca (Justice1) leads the collaboration and all members of the inter-organisational team report directly to her. Rebecca was selected based on her prior experience of running large, multi-disciplinary collaborative ventures. She acknowledges that this collaboration will attract much more media scrutiny and has tighter deadlines than any she has worked on previously. Thus she notes the pressure is formidable.

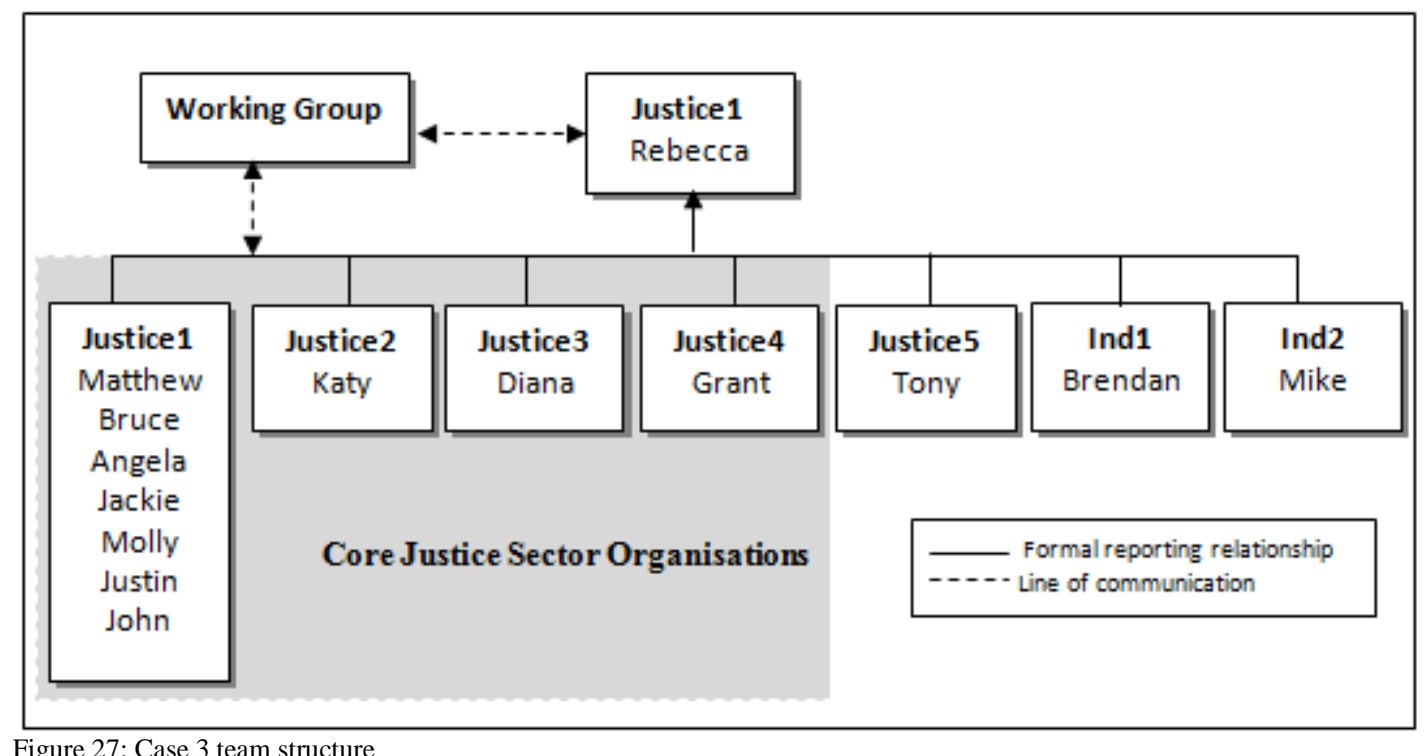

Figure 27: Case 3 team structure 
Other participants within the team are employed in a range of policy and advisoryrelated roles in their respective organisations. Some participants hold more senior positions than others, but in this Case, other than the leadership role, no formal hierarchy is in place. The average tenure of participants in their parent organisations is 6.64 years. Four participants indicated tenures in excess of 10 years within their organisations.

Team members were purposively selected based on their experience and expertise. Rebecca had worked with some individuals previously. In other instances, individuals were recommended or Rebecca was aware of their work through contacts in her wider networks. The majority of participants are engaged full-time in the collaboration, although at least two individuals have only been able to participate on a part-time basis due to pre-existing commitments ${ }^{15}$.

The inter-organisational team also has linkages to a working group comprised of members from a range of organisations associated with aspects of the Case. The working group provides a liaison role between the inter-organisational team and participating organisations. For example, if an organisation wants specific information about the collaboration they can access this through their working group member. The working group member can also connect team members to other specialists within their organisations. The nature of the relationship between the core collaboration team and working group is reciprocal, and the working group provides a key brokerage facility to Case members.

The collaboration is guided by formal terms of reference but, other than this, there is no official documentation that directs how work on the collaboration should be undertaken. The onus is on individuals to take responsibility for their roles in the collaboration and to undertake associated activities based on their experience and expertise. The specific characteristics of the Case are listed in Table 39.

\footnotetext{
${ }^{15}$ Identity of these participants was not revealed.
} 


Table 39: Case 3 characteristics
\begin{tabular}{|l|l|}
\hline Characteristic & Case 3 \\
\hline Physical Type & Co-located \\
\hline Number of Organisations & $5+2$ independent advisors \\
\hline Number of Individual Participants & 14 \\
\hline Duration of Collaboration & Six months \\
\hline Allocation of Staff Resource & Full-time \\
\hline
\end{tabular}

\subsection{Perceptions of knowledge}

Case 3 participants presented definitions of knowledge that were similar and consistent across the team. Textual analysis of the definitions identifies that participants perceive knowledge as contextual, to be associated with individuals' experience and understanding, and for value to be derived from its application.

The contextual nature of knowledge provides individuals with a specific reference point or connection to a specific body of knowledge that enables them to participate in an activity such as an inter-organisational collaboration:

Knowledge is the ability to understand, use and contextualise information.

The set of understandings I have about the Ministry, government processes, the law - the things that give me a context for this collaboration.

Context is also associated with the application or use of knowledge, and where this is able to be communicated effectively, can lead to others' being able to use, apply, or understand that knowledge:

A combination of information and experience not necessarily documented in one place. Knowledge requires ability of a person to communicate relevant information in context in a meaningful way so that others may use it or be able to understand it.

Experience is also considered a key aspect of knowledge, and participants identify that receipt of knowledge can expand an individual's experience, while an individual's experience can contribute to bodies of knowledge that, in turn, are shared with others.

The ability to transfer data into information relevant for decision and the ability to make decisions that guide future actions, using learning and experience. 
A detailed understanding derived from information and experience.

Participants in this collaboration have been purposively selected based on the knowledge, experience, and expertise that they contribute to the Case. In several cases, participants have acquired a vast range of knowledge in terms of the justice sector and its operations, as well as specialist knowledge pertinent to their particular area of expertise. This knowledge is considered critical to the collaboration, particularly because the six month timeframe means that there is no time for participants to learn onthe-job; rather they must be fully operational and able to contribute from the outset.

\subsection{Knowledge sharing activities}

Case 3 identifies as knowledge intensive, where a high degree of sharing is necessary to achieve the collaboration goals. Knowledge sharing is influenced by organisational factors such as the timeframe of the collaboration and the physical proximity of participants. Individual factors such as familiarity with others and preferred communication styles also influence knowledge sharing in Case 3.

The main knowledge sharing activities support the requirement for the team to provide the government with recommendations regarding a key justice issue. This is enacted through the development of specific papers for Cabinet that detail potential new approaches to the issue. The development of these papers is dependent on both the individual areas of expertise that participants bring to the collaboration, and the collective knowledge that is developed from the combination of this expertise.

A majority of participants agree that they are actively encouraged to share knowledge with other team members, and eighty percent of participants expect that knowledge sharing will be reciprocal. It is considered that knowledge is readily shared with others, although a third of Case members noted that some participants share knowledge more readily than others.

Specific organisational factors such as the timeframe of the collaboration and the physical proximity of participants influence the way in which Case 3 members share knowledge. The tight timeframe imposed on the collaboration means that there is little time for non-essential sharing. As a result even informal or casual conversation is likely 
to focus on aspects of the Case, and participants are inclined to focus sharing activity on individuals who will be able to make use of their knowledge. As one participant notes:

I'm not going to spend a lot of time discussing my knowledge on a topic with someone who isn't going to be able to use it.

The physical proximity of the inter-organisational team also plays a role in sharing among team members. The single team space enables all participants to have line of sight with each other. It has access to several small meeting spaces, as well as a larger meeting room. Participants believe that the physical space in which the team is located facilitates spontaneous communication, and this is conducive to knowledge sharing:

I think having everyone around you means you can just lean over a divider and ask for help, or just call out and say 'what do you think about it'.

Participants' familiarity with other Case members and individual communication preference also influence knowledge sharing activity. Most participants indicate that they are more comfortable sharing knowledge with those with whom they are more familiar, although this would not preclude them from sharing with others. Participants express no clear preference regarding the benefits of formal and informal knowledge sharing; although some note that they are more comfortable sharing in informal, rather than formal, situations.

\section{I prefer informal smaller groups than big meetings.}

Knowledge sharing also occurs between the inter-organisational team and the working group that is in place to support the collaboration. Regular face-to-face meetings are scheduled with the working group, and ad hoc, informal, sharing also takes place as and when required. These meetings are held in the inter-organisational team's meeting room and this is seen as helping to establish the identity and purpose of the group:

So we're the team here - come for a meeting here.

The "noise" that occurs in ensuring that all organisations have an opportunity to contribute and communicate their position is seen as a potential downside of knowledge sharing in this and other inter-organisational collaborations: 
Each agency has to have its point of view heard and incorporated and that makes it enormously time consuming and often it is not productive.

There is a high level of interaction, with most participants communicating once a day or more. Much of the driving force for consultation arises from the need for transparency and accountability, and is a particular feature of the public sector where organisations and the government have a high level of accountability to the general public.

\subsubsection{Choice of communication channel for knowledge sharing}

In Case 3, participants have access to a range of communication channels, and knowledge sharing among team members is undertaken through a variety of formal and informal mechanisms including face-to-face communication, email, and telephone. Participants also have access to a shared information repository and a shared workspace. The choice of communication channel is influenced by the physical proximity of case participants, the nature of the communication, and the availability of individuals.

Participants were asked to identify the communication channels they most commonly use to communicate with each of the other participants with whom they had indicated a knowledge sharing relationship. The results show that face-to-face communication is the preference of the majority of participants (see Table 40). The two external advisors, both of whom are located in different buildings to the inter-organisational team, indicate that they most commonly use email for communication purposes. Email is also used by other participants if face-to-face communication is not possible.

Table 40: Choice of communication channel

\begin{tabular}{|l|l|l|}
\hline Channel & $\begin{array}{l}\text { Total } \\
\text { Interactions }\end{array}$ & $\begin{array}{l}\text { No of } \\
\text { Individuals }\end{array}$ \\
\hline Face-to-face meetings & 68 & 12 \\
\hline Telephone & 0 & 0 \\
\hline Email & 19 & 3 \\
\hline Web-based technology(i.e.shared workspace, electronic collaborationtool) & 0 & 0 \\
\hline Other & 0 & 0 \\
\hline & 87 & \\
\cline { 1 - 3 } & & 0
\end{tabular}

Eighty percent of participants consider that face-to-face communication is an important factor in developing good working relationships. In this Case, there is a high level of informal face-to-face communication and this is largely due to the co-located nature of 
the team. Participants report a high degree of ad hoc, opportunistic sharing and this is supplemented by weekly meetings for the entire team. In addition, Rebecca has one-onone fortnightly meetings with each participant.

Although face-to-face communication is most commonly used, there are instances where the nature of the communication influences individuals' choice of channel. For example, email is particularly beneficial because it provides a mechanism to go back and check details, and enables participants to make progress on a task and move on to other activities:

You can go back to confirm what deadlines where agreed, or whatever.

If they look busy, I'll drop them an email .... I move on to the next issue.

Although participants indicate that, in general, the better they know an individual, the more likely they will be to communicate with them face-to-face (see Appendix 13), this does not appear to influence interactions in this case. While the majority of participants met for the first time on the collaboration, eight individuals indicate that they knew between one and three participants prior to their involvement in this endeavour. In these instances, the established connection resulted from having worked together previously, or from participating in the same business networks. Two participants also indicate that they knew each other through non-work related connections. An analysis of frequency of interaction and familiarity with others shows no evidence of higher levels of interaction between those who had known each other prior to the collaboration. There is a high level of interaction, with most participants communicating once a day or more.

\subsection{The use of information and communication technology (ICT)}

In Case 3, ICT is used for information storage and for communication purposes. These activities are facilitated through the use of a shared drive that serves as an information repository for collaboration-related information, and the use of email and telephone for communication purposes. Participants also have access to a Shared Workspace that is administered by Justice1, but no participants utilise the workspace. 
Seventy-one percent of participants are satisfied with the range of tools available for use in this collaboration, with only seven percent indicating that it would benefit from a greater range of tools (see Appendix 13). However, only fifty percent of the participants consider that the existing tools are effective. This suggests that for participants the effectiveness of ICT tools is more important than increased variety of tools. This is supported by participants' use of ICT tools on the collaboration and opinions (detailed later in this section) regarding their usefulness.

At the outset of the collaboration, it was intended that the shared workspace would be used to support the team as both an information repository and communication tool. The workspace is available to Case participants, but is not used by any individual. This is due to initial difficulties associated with making the workspace operational and has resulted in participants' reliance on the shared drive as an information repository. Rebecca believes that the initial difficulties in setting up the shared workspace led to participants expressing cynicism about it. Rebecca states:

It [shared workspace] wasn't ready on time and we got absolutely no training from anybody in-house or anything like that; it was 'you're on your own' and with this project I just haven't had time to nurse people into using it and saying it's there, it's absolutely fantastic, it would be really useful.

Discussion with other participants reveals that, for some, this is not their first introduction to the concept of Shared Workspaces, but previous experiences have resulted in a lack of enthusiasm and doubts about the value of this tool:

I've worked on other projects where we've had shared workspaces. I never went into them because there was this big procedure to login and I had to remember and go and check for updates.

We do have a shared workspace...but it hasn't been used much, and isn't working particularly well I think.

Workspaces are perceived by participants as difficult to use and this has led to participants choosing not to use them. However, this contrasts with Rebecca's positive experience and her enthusiasm for workspaces. One reason for these contrasting views 
may relate to individuals' levels of technical prowess, as well as a lack of formal instruction about the workspace. As one participant notes:

I didn't actually have the time to learn how to use it [the workspace] properly, so while it could be useful, it can create short term barriers. Then you find peopleto-people workarounds and you don't need the technology so much.

The shared drive is managed and maintained by Justice 1 and is used by all participants to store Case related information - although one participant notes that she also keeps information on her personal drive as it is quicker to find and still available if the shared drive crashes.

ICT also plays a role in facilitating communication between participants, and is particularly useful for communication between members of the team who are not located in the same physical location. Access to email is facilitated through Justice1, which has provided email addresses for each participant. Participants indicate that, in most cases, they still have access to their email accounts in their parent organisations, but that collaboration-related communications are directed through the Justice1 email system. Email is most often used by the external advisory members of the Case. For other participants, email use is mostly limited to when participants are not available for face-to-face communication, or when the nature of the interaction requires written communication. Fifty percent of participants do not consider that emails help to establish trust between individuals. One participant believes that it could be useful in building relationships that have already been established:

I think technology [email] can be used to build relationships that exist.

Another participant believes that email can assist relationship building, but cannot provide the depth of communication that establishes strong working relationships, and it is easy for emails to be misinterpreted:

I'm very conscious that you can dash off an email because you're in a hurry and you don't stop and think about how you said something and it can be taken the wrong way. 


\subsection{Social capital}

This section explores SC and how it influences knowledge sharing in Case 3.

\subsubsection{Relational social capital}

Participants place a high degree of importance on aspects of relational SC, as evidenced through both the interview data and the data collected in the Phase 2 survey.

\section{Identity}

Case 3 participants demonstrate a strong sense of identity both in relation to the interorganisational team, and to their individual organisations:

We share a goal...I think making the effort is worth doing...people [in this team] really respond to it [connecting with others].

We're doing [this collaborative] work and everything else comes second.

This sense of identity appears to be connected to the nature of the work in which they are involved, and is a consequence of participants' strong sense of commitment to the collaboration, the intrinsic value of the work that it is being undertaken, and purposeful action on behalf of Rebecca, the team leader.

Table 41 details the factors that motivate Case participants to share knowledge. The high levels of commitment to the collaboration act as the primary motivator for knowledge sharing, as indicated by eleven of the fourteen participants.

Table 41: Motivation to share knowledge
\begin{tabular}{|l|c|c|}
\hline Motivation & $\begin{array}{l}\text { Total } \\
\text { Count }\end{array}$ & Individuals \\
\hline Trust in the individual & 18 & 5 \\
\hline The feeling I am able to help that person & 3 & 2 \\
\hline The belief that the recipient will share their knowledge with me in return & 5 & 2 \\
\hline Commitment to the collaboration & 56 & 11 \\
\hline Presence of technology that makes sharing easy & 0 & 0 \\
\hline Recognition of my own knowledge and expertise & 0 & 0 \\
\hline
\end{tabular}


Rebecca's leadership, and decisions taken by her, have also contributed to the strong sense of group identity. At the outset of the collaboration, Rebecca took several decisions that have assisted the development of each individual's sense of belonging and commitment to the venture. The first decision concerns the co-location of team members. Rebecca considers this to be a critical aspect of the collaboration, enhanced by the fact that the shared team space does not belong to Justice1, the lead organisation:

Essentially it's an independent area, independent of the influence of Justice and their way of doing things...it breaks down any of those inter-agency things.

The second decision relates to the selection of team members. Although Rebecca was not able to get all the participants she would have ideally selected, she was clear about the importance of getting the right people with the right experience and expertise. She considers it is important that participants are individuals who are able to focus on the goals of the collaboration, rather than act as the voice of their parent organisation:

It's about having people on board to focus on the problem at hand and try to avoid them representing their organisation. So they're here for their own expertise rather than as representatives of their organisations.

In the majority of cases, individuals' inclusion in the collaboration has arisen from voluntary commitment in response to organisations calling for volunteers for the interorganisational team. In one instance, a member was co-opted onto the team having returned from leave. At the outset, this individual considered that this was because the organisation was unsure what to do with her, but as the collaboration progressed she came to the conclusion that her inclusion in the team was based on the contribution that she was able to make to the group, and that this contribution differed to the contributions that other team members make. Participants noted:

I wouldn't say I'm representing Justice2. I'm on the team to help the project [collaboration].

Ifeel like I'm a making a real contribution to what's being achieved here.

Only one participant voiced a different opinion. In Diana's case, the loss of organisational identity was a key concern, to the extent that she requested to be lent to 
the collaboration rather than formally seconded. She believes that this results in a different experience:

The difference is maintaining my identity as representing Justice3's interests as against being taken over by the over-riding membership of this project, which is Justice1.

Considering that the Case is not only led by Justice1 and also involves several members of Justice1, it seems reasonable for Diana to assume that the collaboration would reflect the identity and influence of Justice1. However, as this analysis identifies, participants from Justice1 were employed across a number of different business units and most did not have previous knowledge of each other. This suggests that, despite the presence of group norms and individuals' sense of obligation and expectations, the intellectual capability of the group ensures robust debate and discussion that avoids the type of groupthink that can occur when a strong sense of identity is forged between team members. It is also likely that Rebecca's short tenure with Justice1 means she has not yet taken on the identity and culture of Justice1, and is unfamiliar with other team members. As a consequence, no one organisation appears to influence the identity of the group.

Rebecca's focus on team building, and support for the initial development of relationships between individuals has also helped the team to forge a sense of identity. At the outset of the collaboration Rebecca held an informal social function at her home to enable participants to socialise and get to know each other. As the collaboration has progressed, some participants choose to get together for drinks on Friday evenings, and Rebecca provides lunch when the team needs to meet during a lunchtime period, which sometimes happens when there is no other time available.

A strong sense of self, as exhibited by the individuals interviewed in this Case, has also positively influenced the strong sense of group identity. Interview participants are aware of how they, as individuals, can contribute to the collaboration, and believe that the combination of their individual expertise provides an advantage in ensuring that each paper developed on the collaboration benefits from the different strengths of individuals. While a strong sense of group identity can lead to groupthink which can inhibit innovation (Nahapiet \& Ghoshal, 1998), in Case 3 the specific depth and breadth 
of experience has proved to be advantageous to the group by promoting rigorous debate that has been beneficial to the work.

Participants also indicated that they closely identify with their individual organisations. In Diana's case (outlined above) this led her participation in the collaboration to be structured such that she maintained strong links to her parent organisation despite fulltime engagement in the inter-organisational team. Other participants have worked within the parent organisation, or the justice sector, for periods in excess of 10 years during which they have built up a high degree of specialist knowledge. The work they undertake within their parent organisations relates to specific aspects of the justice system and has enabled them to become experts in these areas. This suggests that it is the particular justice-related aspects of the work to which they are connected, rather than the generic area, research, that drives this sense of identity and retains them in the respective organisations.

\section{Trust}

Trust is an important issue for Case 3, and is related to other aspects of SC, such as the sense of group identity, and agreement about the purpose and goals of the collaboration and how these can be achieved. Participants indicate that trust has the potential to be the greatest barrier to knowledge sharing in public sector inter-organisational collaboration (see Appendix 13). In this Case, trust is a key motivational factor in encouraging individuals to share their knowledge with others (see Table 41 above).

All participants consider trust to be an important aspect of collaboration, and sixty-four percent of participants perceive the level of trust between Case participants to be above average. (see Appendix 13). This level of trust appears to derive from the sense of commitment that individuals feel toward the collaboration and the value of the work it is undertaking, as well as the fact that they are trusted to do their job and are not constantly monitored:

I've found that the best policy is where you test your ideas out on your workmates and others, because the more minds you get around a problem, the better the solution. 
She [Rebecca] just lets you get on with it. She is an incredibly strong leader; she's also remarkably effective and really got the best out of everybody.

Given the strong sense of identity shared by participants, the researcher expected that a greater proportion of participants would consider trust levels to be above average. Further analysis of the survey data indicate that while Justice1 participants perceive trust levels to be above average, for the most part, this sense of trust was not shared by participants from other organisations. This suggests that individuals from the same organisation are more likely to trust each other, even if they do not work within the same business units.

Case participants indicate that trust can be built in several ways such as through individual competence, but do not identify any one method as better than others. Interviews with participants reveal that individual competency is important in this Case because, as one team member notes, individuals need to "hit the ground running". However, some participants also believe that getting to know other team members is important. Relationship building is something that Case members consider can occur during the course of the working day, and does not need to be specifically engineered through social functions or events outside of the workplace. However, it does require an investment on behalf of the individual, and is assisted when individuals are colocated:

It's like an investment really. You invest a bit and it pays off.

We spend a lot of time just chatting and to me that's not wasted time. It's developing the group and often the chat is work-related, and I think from that point of view this team's moulded well. There's not clear exclusion of people.

There is value in sitting round the tea table because generally talk is about work and it develops.

Some participants suggest that Rebecca trusts some team members more than others, and that those who are more highly trusted are assigned specific activities that other team members are not involved in: 
I picked the two or three people that she just had complete trust in and those people tended to pick up a lot of the more difficult parts of the project or the external relationship management that wasn't being handled by her.

There may be several reasons for the perception that Rebecca trusts some more than others, including pressure to deliver collaboration outcomes within a short timeframe, and previous experience working with others:

In this project I couldn't afford to hand hold.... You just have to have a certain trust in their [member's] professional ability that they will get the job done.

Given the pressure on Rebecca and the team to deliver the required outcomes within a short period of time, it is possible that she will be more confident that those with whom she has previous experience of working will be able to accomplish a specific task. Her purposeful selection of these participants indicates an a priori level of trust in these individuals.

Several participants agreed that there is a degree of "noise" that occurs at the outset of a collaboration, while individuals are working out where they fit in the interorganisational team and whether they are able to trust participants from other organisations. However, the accumulated experience of working in several multiagency collaborations can also help to build trust between individuals from different organisations:

With multi-agency projects such as this, you do get to a position after you've been operating for a while with the organisations, to trust one and other and that makes it easier.

In summary, trust is an important issue in this Case, and has been contributed to by a range of factors including the combination of the specific decisions and actions taken by Rebecca in relation to the co-location of team members, team member selection and team building activity; the collaboration timeframe; the strong sense of group identity; and the perceived value of the work in which they are engaged. 
Norms, obligations and expectations

Collaboration constraints (specifically, the timeframe); expectations of individual competence; and levels of commitment to the collaboration all contribute to the development of behavioural norms, and obligations and expectations that influence knowledge sharing in this Case.

The non-negotiable conditions of the collaboration, such as time constraints, have lead participants to develop unwritten norms and expectations regarding the way in which work is undertaken, and has influenced individual's propensity to share knowledge among team members. As previously mentioned, Rebecca's expectation is that individuals can operate effectively with minimal guidance; this is confirmed by team members who expect that other participants will deliver the tasks they have been assigned.

There are no guidelines that outline how work in the collaboration should be undertaken, and Rebecca does not consider that hands-on project management is necessary when working with experienced individuals. This has resulted in Case participants accepting responsibility for tasks with the expectation that they will complete the task by the most effective and efficient method, and has established a strong sense of co-operation.

"There is a bit of reliance on individuals and the backgrounds they bring in terms of working in these sorts of areas and the day to day decisions they make. So it's about counting on what we have rather than a manual of do's and don'ts."

Individuals' levels of commitment to the collaboration also influence knowledge sharing in the Case and act as an implicit obligation on participants to contribute to the collaboration, and the expectation that others will do the same.

\subsubsection{Cognitive social capital}

The analysis of cognitive SC focuses on issues of shared language, shared purpose and goals, and a sense of shared culture. 
Shared language

In this Case, analysis of the research data does not reveal any significant issues, either positive or negative, that relate to shared language. Although participants note that language can be an issue for inter-organisational collaboration, they do not believe that it is an issue for this Case:

You get other agencies who don't like what you're saying to them...I think you can do a certain amount by being neutral with the language that you use.

They all want to be completely consistent with everything else and with their own ministers. So there's quite a lot of wording things and it's not, it's often not about the core substance.

In Case 3, possible reasons for the lack of concern regarding language are the nature of the work that is being undertaken, and the ability of individuals to easily consult with others as required. As most participants are employed within policy roles, it is likely that while their specific areas of expertise may vary, the generic language of policy does not. On occasions where differences in terminology may be present, the co-located nature of the team enables these differences to be discussed at the time that they arise, and so are not identified as issues with the Case.

\section{Shared purpose and goals}

The purpose of Case 3 is to develop a range of papers that inform and make recommendations for new approaches to an existing justice issue. This purpose was clearly understood and supported by participants:

\section{I'm on the project to help the project}

We have a shared goal.

This sense of shared purpose is supported by the earlier findings relating to the strong sense of group identity and commitment to the work of the collaboration (see Table 41). In addition, the purposeful selection of team members at the outset of the collaboration helped to ensure they were likely to be predisposed to the goals of the venture, and this careful preparation has contributed to the collective sense of purpose. In most instances, 
participants believe that the collaboration is addressing an important issue and they indicate that they want to contribute to finding better solutions:

There was a remarkable level of gelling between people and I think a lot of that was down to people just being willing to get down and do the job which I think says a lot about the team.

This suggests that specific attention to the selection of team membership may help agreement of shared goals and limit the presence of individual agendas.

\section{Shared culture}

Fifty-seven percent of Case participants consider the culture of the inter-organisational team to be different to the culture in their own organisations (see Appendix 13). Analysis of this response indicates that the differences between group culture and individual organisational culture are predominantly observed by participants who are not members of Justice1. In fact, the majority of Justice1 members chose not to respond to this question.

Participants also show a high level of optimism in regard to the ability to blend different organisational cultures within an inter-organisational team. More than fifty percent of participants considered that blending cultures is not an issue for inter-organisational teams. However, analysis identified that the majority of Justice1 participants disagree with other participants and consider that blending cultures in to a single interorganisational team can be problematic.

The responses of Justice1 members to questions relating to culture suggest that this may be an area of difficulty, although it is not clear whether this relates to issues within the inter-organisational team, or is indicative of organisational level issues.

Consideration of individual comments relating to this issue suggests that the culture within Justice 1 is different to the other participating organisations, and may be more formal than other cultures:

Justice5 has a pretty strong intellectual drive...collegiality, and an open working environment and relationships. 
I find that Justicel are very cautious; they're very slow to act .... And they're very hierarchical.

Although the differences in organisational culture are acknowledged, they do not seem to have an influence on the working of the inter-organisational tam. In fact, the findings from this Case demonstrate a tangible absence of hierarchy and process:

I think there is a view of ad hoc to it [the collaboration]; I don't see that as a bad thing. I don't know that a manual is something that would add a lot but that's a judgment on the skills of the people and the somewhat informal mechanisms and mechanisms that we have within the project.

To be frank, I deliver...so a lot of projects like this end up with me.

Though it is difficult to derive specific meaning from these results, together they show a pattern of difference between perceptions of participants from Justice1 and participants from other organisations in regard to the issue of shared culture. This pattern has not been noticeable in other aspects of the Case. However, given that Justice1 is the lead organisation in the collaboration, and the overall management of the team falls to Justice1 team member Rebecca, it might be expected that Justice1 members would perceive the inter-organisational team culture to be reflective of their organisational culture. However, this is not the case. One reason for this may relate directly to Rebecca and her leadership of the collaboration. Rebecca has only recently joined Justice1, and was identified to lead the inter-organisational team only two weeks into her tenure. Thus, it is reasonable to surmise that she had not yet had sufficient time to become embedded in the organisational culture of Justice1.

\subsubsection{Structural social capital}

Structural SC focuses on the formal structure (who reports to whom) and the informal structure (who interacts with whom). The formal structure of the inter-organisational team was detailed in Section 6.1. The collaboration is characterised by a lack of formal processes and procedures, and relies more on the experience and expertise of individuals to deliver to the collaboration goals. Despite the involvement of individuals with different levels of experience and at different steps on their respective hierarchical ladders, there is no formal reporting hierarchy within the collaboration other than to the 
team leader. These findings indicate that, overall, the collaboration operates on a relatively informal basis.

Figure 28 presents the informal network identified in Case 3. The data shows a highly cohesive network with high levels of interaction between individuals. There is a strong degree of reciprocity within the network and interaction between individuals is frequent. Despite the large proportion of Justice1 participants in this Case, the visual network shows that while three Justice1 members hold strong places within the network, several are located in more peripheral positions and, as such, Justice1 members do not dominate the network. Eight cliques exist in the network, with several overlaps between memberships of these cliques. There are no cutpoints within the network indicating that no individual is wholly dependent on another member for connecting to other actors. A full analysis of the meaning of the cliques with the Case 3 networks is detailed later in this chapter. 


\begin{tabular}{|lll|}
\hline & Justicel & Unreciprocated tie \\
Justice2 & Reciprocated tie \\
Justice3 & Justice4 \\
Justice5 & Ind1 \\
Ind2 & \\
\hline
\end{tabular}

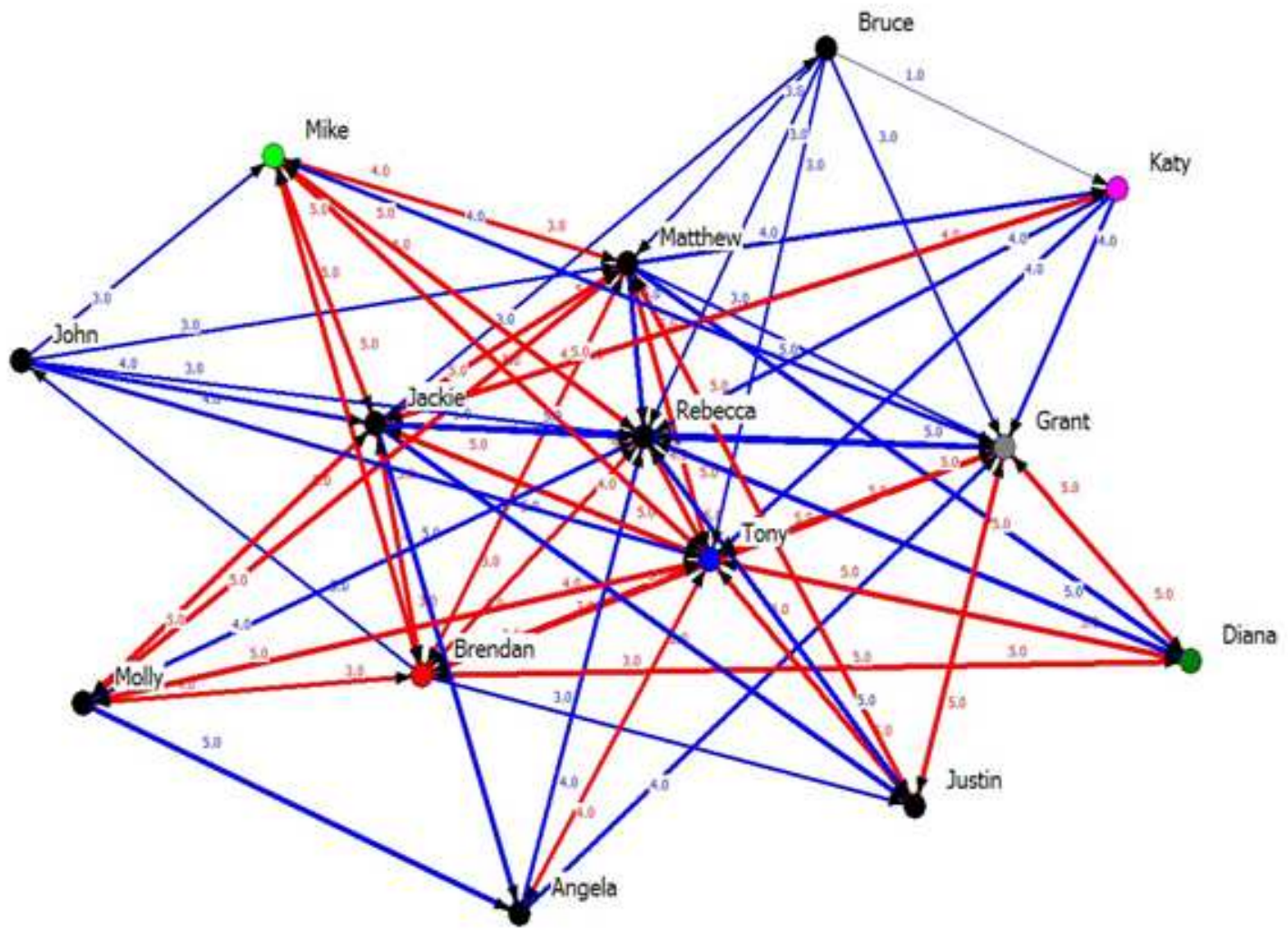

Figure 28: Case 3 informal network

\subsubsection{Network level results}

Table 42 provides an indication of the health and overall cohesion of the network.

(Extended descriptions of these measures can be found in Chapter 3, Section 3.3.4.2.)

Table 42: Case 3 network level measures

\begin{tabular}{|l|l|}
\hline Measure & Result \\
\hline Size & 14 \\
\hline Total Ties Present & 84.0000 \\
\hline Density (Mean) & 0.4615 \\
\hline Distance & 1.610 \\
\hline Std Deviation & 0.499 \\
\hline Variance & 0.249 \\
\hline
\end{tabular}

The statistics presented in Table 42 provide an indication of the health and overall cohesion of the network. (Extended descriptions of these measures can be found in Chapter 3, Section 3.3.4.2.) 
These statistics represent a highly cohesive network where information flows quickly to network members and there is a high level of SC between Case participants. Both the network density (46\%) and distance measures (1.610) suggest this is a highly effective network and there are likely to be few bottlenecks or impediments to information flow. The network density shows a high level of connections among team members with 84 ties, (of the 182 possible ties) in place across the network. This is facilitated by the relatively low number of participants within the network, and it is likely that network cohesion would decrease if more members were to join the inter-organisational team.

\subsubsection{Individual level results}

Degree, closeness and betweenness centrality measures were selected to analyse the ties between individuals in the network. (Full descriptions of these measures can be found in Chapter 3, Section 3.3.4.2.)

\section{Degree centrality}

Degree centrality measures the number of direct connections an actor has. A full list of normalised degree centrality degree ${ }^{16}$ scores for Case 3 is shown in Table 43.

Table 43: Case 3 degree centrality measures

\begin{tabular}{|l|l|l|l|r|r|r|}
\hline $\begin{array}{l}\text { Centrality } \\
\text { Measure }\end{array}$ & Actor & $\begin{array}{l}\text { Formal } \\
\text { Role }\end{array}$ & Organisation & NrmOutDeg & NrmInDeg & NrmDegree \\
\hline+ & Rebecca & leader & Justice1 & 23.077 & 100 & 100 \\
\cline { 2 - 7 } & Tony & member & Justice5 & 76.923 & 84.615 & 100 \\
\cline { 2 - 7 } & Jackie & member & Justice1 & 84.615 & 53.846 & 92.308 \\
\cline { 2 - 7 } & Matthew & member & Justice1 & 69.231 & 61.538 & 92.308 \\
\cline { 2 - 7 } & Grant & member & Justice4 & 38.462 & 76.923 & 84.615 \\
\cline { 2 - 7 } & Brendan & member & Ind1 & 61.538 & 69.231 & 76.923 \\
\cline { 2 - 7 } & Justin & member & Justice1 & 30.769 & 38.462 & 53.846 \\
\cline { 2 - 7 } & Mike & member & Ind2 & 46.154 & 46.154 & 53.846 \\
\cline { 2 - 7 } & Bruce & member & Justice1 & 38.462 & 7.692 & 46.154 \\
\cline { 2 - 7 } & Diana & member & Justice3 & 30.769 & 23.077 & 46.154 \\
\cline { 2 - 7 } & John & member & Justice1 & 46.154 & 7.692 & 46.154 \\
\cline { 2 - 7 } & Katy & member & Justice2 & 30.769 & 23.077 & 46.154 \\
\cline { 2 - 7 } & Molly & member & Justice1 & 46.154 & 30.769 & 46.154 \\
\cline { 2 - 6 } & Angela & member & Justice1 & 23.077 & 23.077 & 38.462 \\
\hline
\end{tabular}

\footnotetext{
${ }^{16}$ Normalised scores divide simple degree by the maximum degree possible and enable cross case analysis to be undertaken on networks of different sizes.
} 
The NrmDegree centrality measures indicate several interesting findings. Firstly, the measures show a large variance between the highest and lowest scores of actors. Justice1 members hold both the lowest and highest degree centrality scores. Angela has the lowest degree centrality score and is likely to find it more difficult to source information than the majority of actors in the Case. The two highest scores were recorded by Rebecca and Tony. This score reflects Rebecca's role as the team leader, within the formal network. The more interesting finding relates to the relationship between Rebecca and Tony who, until recently, worked together in Justice 5. The informal network reflects the strong tie between the two and also provides support for comments from participants who observed that Rebecca sometimes gives greater responsibility to team members whom she knew prior to the collaboration.

The degree measures also indicate that, despite the fact that both Brendan (Ind1) and Mike (Ind2) are not co-located within the team, this does not affect the flow of information to and from these individuals. This provides further support for the qualitative finding that, although Justice1 members comprise the greater component of the inter-organisational team, they do not dominate the network.

Analysis of actors' in-degree and out-degree measures provided more insight into the network. The variance between the in-degree scores of individuals was measured at 13.286 (see Appendix 14), showing the level of difference between the number of information requests received by actors as evidenced by the difference between the highest number of information requests received by Rebecca (100), and the lowest number of requests received by both John and Bruce (7.692).

Out-degree scores indicate a lower level of variance (6.143), and indicate that both Jackie and Tony provide key support to Rebecca's leadership role. In contrast to the number of information requests received by Rebecca, she rarely seeks information from Case participants other than Jackie and Tony who act as brokers on her behalf. The greatest number of information requests are made by Jackie, who is the sole administrator for the team and who also operates as Rebecca's personal assistant. In this respect, she is often charged with obtaining information from Case participants for Rebecca, and this is evidenced through her high out-degree score. Tony also made a high number of information requests (76.923). In addition to his role as a participant within the collaboration, Tony describes his role as: 
Being involved in co-ordinating the whole shooting box...to organise people so that everyone knows who's doing what paper and that sort of thing.

Tony's high in-degree and out-degree scores suggest that he occupies a senior role in the team whereby he is the unofficial deputy team leader. This is probably due both to Tony's seniority within his parent organisation, and the fact that he and Rebecca have previously worked together. Prior to her appointment at Justice1 she also worked at Justice5, the organisation to which Tony belongs.

Network centralisation was also measured as an additional point of reference for the social network data. Network centralisation measures the global or macro level centralisation of the network and shows whether or not it is centred on specific individuals.

In this Case, network centralisation was derived from the degree centralisation measures (see Table 44). The overall degree centralisation index (41\%) indicates a highly centralised network where activity is largely focused around a subset of network actors.

Table 44: Case 3 network centralisation index

\begin{tabular}{|l|l|}
\hline Centralisation Index & Result \\
\hline In-degree & $57.988 \%$ \\
\hline Out-degree & $41.420 \%$ \\
\hline Total degree centralisation & $41.03 \%$ \\
\hline
\end{tabular}

Specific analysis of in-degree and out-degree centralisation indicates a greater centralisation on incoming information requests, suggesting that some individuals occupy positional advantages in the network, and that power amongst individuals varies substantially. This correlates with the qualitative findings, presented earlier in the study, that certain individuals within the team are assigned more complex tasks, or tasks that require a higher degree of trust. This can also be seen in Figure 28 which shows a small number of actors who appear more centrally within the network and who receive a greater number of requests for information than other actors.

\section{Closeness centrality}

The closeness measures for Case 3 are listed in Table 45 . 
Table 45: Case 3 closeness centrality measures

\begin{tabular}{|l|l|l|l|r|}
\hline $\begin{array}{l}\text { Centrality } \\
\text { Measure }\end{array}$ & Actor & $\begin{array}{l}\text { Formal } \\
\text { Role }\end{array}$ & Organisation & TotalCloseness \\
\hline+ & Tony & member & Justice5 & 167.917 \\
\cline { 2 - 5 } & Jackie & member & Justice1 & 155.088 \\
\cline { 2 - 5 } & Rebecca & leader & Justice1 & 152 \\
\cline { 2 - 5 } & Brendan & member & Ind1 & 148.693 \\
\cline { 2 - 5 } & Matthew & member & Justice1 & 148.693 \\
\cline { 2 - 5 } & Grant & member & Justice4 & 137.772 \\
\cline { 2 - 5 } & Mike & member & Ind2 & 130 \\
\cline { 2 - 5 } & Molly & member & Justice1 & 124.091 \\
\cline { 2 - 5 } & Justin & member & Justice1 & 116.072 \\
\cline { 2 - 5 } & Diana & member & Justice3 & 110.689 \\
\cline { 2 - 5 } & John & member & Justice1 & 109.828 \\
\cline { 2 - 5 } & Angela & member & Justice1 & 106.522 \\
\cline { 2 - 5 } & Katy & member & Justice2 & 104.67 \\
\cline { 2 - 5 } & Bruce & member & Justice1 & 102.424 \\
\hline
\end{tabular}

These results reinforce the strength of the roles of Tony, Jackie, and Rebecca within the network and show that their positions enable them to reach other actors with relative ease. By comparison, actors such as Bruce and Katy are likely to find it more difficult to reach actors to whom they are not directly connected. However, it should be noted that this network demonstrates high closeness measures indicating that even those with lower closeness measures have the ability to reach other actors with ease. In this Case, physical proximity does not make a difference to actors' closeness scores, as the two independent advisors received higher closeness scores than certain other co-located team members. As seen earlier in the analysis of degree centrality scores, Justice1 participants represent both the highest and lowest scoring actors, providing further evidence of their lack of dominance with the network.

\section{Betweenness Centrality}

Betweenness centrality measures for Case 3 are listed in Table 46. 
Table 46: Case 3 betweenness centrality measures

\begin{tabular}{|c|c|c|c|c|}
\hline $\begin{array}{l}\text { Centrality } \\
\text { Measure }\end{array}$ & Actor & Formal Role & Organisation & nBetweenness \\
\hline \multirow[t]{14}{*}{+} & Tony & member & Justice 5 & 21.923 \\
\hline & Jackie & member & Justice 1 & 14.83 \\
\hline & Brendan & member & Ind1 & 14.231 \\
\hline & Matthew & member & Justice 1 & 9.338 \\
\hline & Grant & member & Justice 4 & 4.467 \\
\hline & Rebecca & leader & Justice 1 & 3.078 \\
\hline & Mike & member & Ind 2 & 1.368 \\
\hline & Molly & member & Justice 1 & 0.813 \\
\hline & Justin & member & Justice 1 & 0.514 \\
\hline & Katy & member & Justice 2 & 0.294 \\
\hline & Angela & member & Justice 1 & 0.128 \\
\hline & John & member & Justice 1 & 0.092 \\
\hline & Diana & member & Justice 3 & 0.08 \\
\hline & Bruce & member & Justice 1 & $\underline{0}$ \\
\hline
\end{tabular}

The results identify that Tony holds the most strategic position in the network. He is highly sought out by team members, and has a positional advantage over other actors that enables him to apply some degree of control of the flow of information in the network should he so choose. This is further confirmation of the role that Tony plays in supporting Rebecca and as the central co-ordinator of the work that is carried out by the team. Tony's role as intermediary also allows Rebecca to undertake other responsibilities of her role that require her to dedicate a large amount of time to meeting with parties outside the immediate team, and to provide a direct line of reporting to the Prime Minister and others.

She [Rebecca] does a lot of the networking, presenting to the high up and the hierarchy stuff; she does all that communication and relationship management stuff.

As has been observed in other centrality measures, several members of the interorganisational team (Molly, Justin, Katy, Angela, John, Diana, and Bruce) occupy more peripheral roles in the network. Their outlying positions suggest that these individuals would find it more difficult to exert influence within the network and reinforces the finding that, in this Case, organisational membership does not positively influence ties between actors. 


\section{Cliques}

Eight cliques were identified in the network as shown in Table 47. Cliques represent subsets of the network in which actors are more intensely linked (Hanneman \& Riddle, 2005). Statistics relating to these cliques are included in Appendix 14.

Table 47: Case 3 cliques

\begin{tabular}{|l|c|c|c|c|}
\hline Cliques & \multicolumn{4}{|c|}{ Members } \\
\hline Clique 1 & Diana & Grant & Tony & \\
\hline Clique 2 & Grant & Justin & Tony & \\
\hline Clique 3 & Jackie & Mike & Tony & \\
\hline Clique 4 & Jackie & Molly & Tony & \\
\hline Clique 5 & Mike & Rebecca & Tony & \\
\hline Clique 6 & Brendan & Jackie & Matthew & Mike \\
\hline Clique 7 & Brendan & Jackie & Matthew & Molly \\
\hline Clique 8 & Brendan & Mike & Rebecca & \\
\hline
\end{tabular}

The presence of these cliques was an unexpected finding as high density networks are often found to have few subgroups (Hanneman \& Riddle, 2005). There is considerable overlap in clique membership and Tony is central to five of the eight cliques. A further interesting aspect of these cliques is that they do not represent Justice1 subgroups, as might be expected where there is a dominance of participants from a single organisation. Rather, these cliques are dominated by the lesser represented organisations, and the two independent external advisors to the team.

\section{Tie Strength}

Case 3 is characterised by strong ties. Interaction is frequent, typically more than once a day, and often reciprocal. The overall measure of reciprocity for the network is $42 \%$, indicating that almost half the team enjoys reciprocal ties resulting in a mutual exchange of information and knowledge.

As previously detailed, Case 3 participants are engaged in producing a number of papers related to their specific areas of expertise, as well as an overarching paper that ties together the recommendations put forward by the team. In order to provide a cohesive set of outputs, it is necessary for the team to work together and to ensure that each paper fits within the overall suite of papers that will be delivered through the collaboration as well as the overarching recommendations of the team. The levels of tie strength suggest that these needs are met through a high degree of reciprocal knowledge sharing activity 
between participants. Thus, the inter-organisational team enjoys a high level of robust discussion and debate, and that this is likely to increase the potential value of its outputs.

\subsection{Summary of Chapter 6}

This chapter has presented the key results and analysis of Case 3 of this research. The first section of this chapter presents an overview of the Case including the purpose of the collaboration, and details of the organisations and individuals involved in the venture. Section two presents the findings related to the specific focus of the collaboration - SC and ICT. It details individuals' perceptions of knowledge and the knowledge sharing activities that take place within the collaboration; the way in which ICT is used to support the team; and provides evidence of the way in which aspects of SC manifest within the collaboration. The chapter also identifies the informal network evident within the Case and presents both visual and quantitative measures to describe the configuration of the network and roles of individuals within it.

This Case represents the single instance of a co-located collaboration within this study. The collaboration arose from the need to find solutions to issues in the criminal justice system. The nature of the venture, both in terms of its subject matter, and its strict timeframe, necessitated the recruitment of highly experienced individuals who could be trusted to produce the required results, while working largely unsupervised.

Participants have access to a range of ICT tools, but report some dissatisfaction with tools such as the shared workspace, which they perceive as difficult to use. Overall, the close proximity of team members and the complex nature of the work in which they are engaged means that knowledge sharing and communication is most frequently undertaken through face-to-face activities, such as formal and informal meetings.

The findings indicate a high degree of SC among participants. The inter-organisational team exhibits a strong sense of identity derived from deliberate decisions regarding the formation of the team, as well as a strong sense of individual and organisational identity, and a belief in the value of the work being undertaken.

Comparison of the formal structure derived from the qualitative data and the informal network (derived from the social network data) suggests that the collaboration is more 
reliant on a subset of individuals than is evident from the qualitative data alone. Though, in many instances, strong ties have been found to inhibit innovation, in this Case they have enabled robust debate and discussion that is seen to benefit the Case. 


\section{CASE 4 RESULTS}

This chapter presents the final case in the study. The results are presented following the same format as has been used in previous cases.

\subsection{Overview of Case 4}

This section provides an overview of the structure, purpose, and activities of Case 4. The section also provides details of the organisations and individuals that comprise Case 4.

Case 4 represents a joint health initiative that focuses on improving health outcomes for New Zealanders ${ }^{17}$. The collaboration is commonly referred to as a programme, and is part of a wider stream of work being undertaken in the health sector. The over-arching purpose of the programme is to improve the well-being of people at greatest health risk more quickly than those at lesser risk. This is a large undertaking and the programme has no specific end date; theoretically it could continue indefinitely.

The collaboration is managed and funded by two main organisations (Health1 and Health2). Health1 represent the government interests in the health sector. Health2 is an industry association body that facilitates and coordinates strategic activity across a range of health organisations (i.e. Health3 and Health4) through collaboration and collective activity. The Case also involves also four shared support agencies (Support1, Support2, Support3, Support4) who are owned by their local regional health organisations (i.e. Health3 and Health4), and who support the over-arching role of Health2. Both Health1 and Health2 report directly to the Minister of Health. A full breakdown of the organisations and individuals participating in this Case are depicted in Figure 29.

\footnotetext{
${ }^{17}$ To ensure the anonymity of project team members, specific details relating to the project's purposes are excluded.
} 


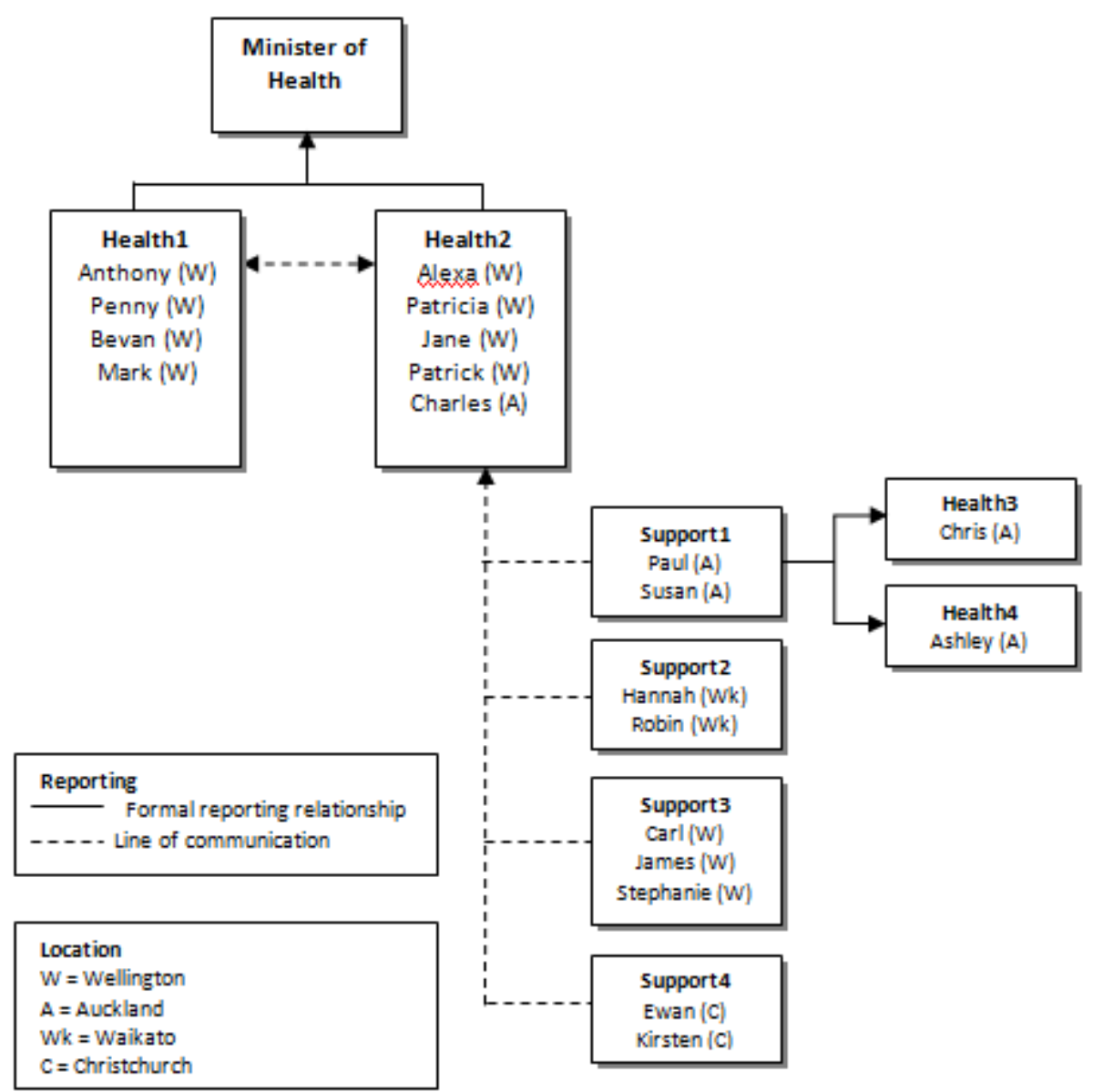

Figure 29: Case 4 formal structure

Both Health1 and Health2 have a programme sponsor whose main responsibilities are to attend to the strategic direction of the collaboration, to maintain a level of pace, and to manage programme related risk. The Health1 sponsor has delegated the day-to-day management of the programme and this is mainly handled by a staff member, Jane. Recent changes mean that Jane is now employed by Health2; however an agreement has been reached by the two organisations to enable Jane to continue to manage the programme on behalf of Health1. The two organisations often differ about aspects of the collaboration, so this continued relationship demonstrates the level of ability and trust that Jane holds within both organisations.

The implementation and coordination of the collaboration is managed by the four Health2 members who are located in Wellington. This team is led by Alexa and is located in a centrally located office in Health2's Wellington premises. Apart from this co-located four person programme team, all other participants work in organisations 
across New Zealand, therefore the majority of contact is through email and telephone. Thus, Case 4 is categorised as a virtual collaboration.

The majority of Case participants are employed in a range of analysis and information related roles such as data and performance analysts. One participant is a clinical leader and two occupy managerial positions. The average tenure of participants in their parent organisations is 3.92 years. Two participants indicated tenures in excess of 10 years within their organisations.

Team members' participation in the Case is related to their roles in their parent organisations. This means that when an individual leaves an organisation, their place on the inter-organisational team is taken by the incumbent employee, or another existing employee. Other than Alexa and Jane who are engaged full-time on the collaboration, all members undertake their activities in conjunction with the other day-to-day tasks and responsibilities of their roles.

Initial funding for the programme was provided jointly by Health1 and Health2 with Health2 accountable for the day-to-day management of the funds. This changed in 2007, when Health1's provision of operational costs was replaced by funding from the four support organisations. Health2 expect that this will mean that Health1 will have less influence in how the programme is managed, although this has not been formally discussed.

The programme has experienced a number of challenges, particularly in the early stages. Initially Health1 and Health2 disagreed about who had decision making powers. As a result, the collaboration now operates with mandated terms of reference and a range of formal processes and procedures that denote decision making powers and guide activities.

The specific characteristics of the Case are listed in Table 48. 
Table 48: Case 4 characteristics

\begin{tabular}{|l|l|}
\hline Characteristic & Case 4 \\
\hline Physical Type & Virtual \\
\hline Number of Organisations & 8 \\
\hline Number of Individual Participants & 20 \\
\hline Duration of Collaboration & Indefinite \\
\hline Allocation of Staff Resource & Part-time $^{18}$ \\
\hline
\end{tabular}

A breakdown of participants, their organisations, and the phase/s of the research in which they participated are shown in Appendix 8.

\subsection{Perceptions of Knowledge}

Many of the knowledge definitions proffered by Case 4 participants focused on learning, application, experience, and understanding. A full list of participants' definitions is detailed in Appendix 9.

Participants consider knowledge to be a personal asset that is accumulated through experience and learning and that can be applied to situations to extend understanding and value of those situations:

Information that I possess on the subject as an individual or the ability to know where it exists and find it. Some of it comes with experience and from reasoning skills.

Knowledge is personal, in that no two people will ever have the same degree of knowledge. It is more than just formal learning; experience and life should be taken into account. Knowledge is for sharing.

These definitions suggest that people acquire knowledge through personal analysis and interpretation of events. In this way, individuals apply their own understanding to develop personal knowledge. In turn, this knowledge can then be externalised or shared with others. This can increase understanding on both an individual and collective basis:

\footnotetext{
${ }^{18}$ With the exception of the two Health2 staff allocated to the project on a full time basis.
} 
Anything you can contribute which will develop someone else's understanding or skills.

Having learnt the end-to-end process of a specific workstream and then being able to share those learnings with others to improve a process, solution of system.

Knowledge is also associated with its ability to contribute to, or enhance decisionmaking. In this respect, it is the way in which knowledge is used and applied that is key:

How information is used to understand problems and inform decision, change behaviours.

Individuals in the collaboration have a range of areas of skill and expertise including knowledge of data and information analysis, knowledge of the health sector, expertise in the development of performance measures, and communication expertise.

\subsection{Knowledge Sharing Activities}

In this Case, knowledge sharing supported a wide range of activities within the interorganisational team and across the health sector. Though this Case focuses on the work of a single team, the collaboration itself forms part of an extensive programme of work across the sector and with private health organisations; therefore many of the knowledge sharing activities undertaken by the team have an impact on the wider health community.

The main focus of the collaboration is on the collection and analysis of data gathered from health organisations across New Zealand, and the development of specific indicators that enable the data to be measured and interpreted:

It is a lot more about information sharing than it is about knowledge sharing. As we get smarter we start to maybe transition into the knowledge sharing than the information.

Therefore, information is considered a critical element of the collaboration, and individuals from outside the inter-organisational team often perceive this as technical- 
information related work. However, this is not regarded by participants as a technical endeavour:

It's not a technical project, but people get caught up in the technical details...they can show their knowledge [about the technical detail], but it actually doesn't change the way they practice medicine and the way that organisations work.

So, while the development of accurate indicators is an essential feature of the collaboration, the accurate interpretation of the data, and the sharing of the data with the wider community also relies on the specific knowledge and expertise of Case members. In this way, the diverse range of participants' skills and expertise supports the array of activities that are undertaken. For example, the extensive interaction with health organisations members such as doctors, clinicians, and health managers requires strong communication skills and generic understanding of the health sector.

The outcomes of the collaboration are also used to inform, and contribute to, the development of policy within the health sector. While not all of these activities are undertaken by the core inter-organisational team, the work in which they are engaged often contributes to the wider picture, and requires interaction and knowledge sharing with a wide range of individuals and organisations. As two participants note:

A lot of what I do is talking to people within the health sector.

My key responsibilities are relationships, communications...

The majority of participants agree that they are actively encouraged to share knowledge with other team members, and eighty percent of participants do so with the expectation that the recipient will share knowledge with them in return. There is little variation among individuals in terms of their willingness to share knowledge; individuals believe that, overall, knowledge is willingly shared by inter-organisational team members.

Eighty percent of participants indicate that they are more comfortable sharing knowledge with participants with whom they are more familiar. Analysis of individuals' relationships with other Case participants indicates that while most of them "met" for the first time after being assigned to the collaboration, in a small number of 
instances, participants had previously worked with other individuals or were aware of them through their involvement in the same business networks.

\subsubsection{Choice of communication channel for knowledge sharing}

In Case 4, participants have access to a limited range of communication channels. All participants have access to email and telephone, but face-to-face communication is limited to those who work in close proximity to each other. However, due to the nature of their co-ordination roles, both Jane and Alexa undertake frequent travel throughout New Zealand and are more likely to be able to communicate on a face-to-face basis with other participants. The choice of communication channel is influenced by the physical proximity of case participants, the nature of the communication, the purpose of the communication, and the relationship with the recipient.

Face-to-face communication and email are the most popular choices of communication channel (see Table 49). However, as indicated above, physical proximity influences the ability for face-to-face communication; therefore it is likely that the majority of those who select face-to-face meetings as their most common communication channel are from the core team who are centrally co-located, or are from the same parent organisation. Conversely, for participants who are located in different cities, knowledge sharing is more commonly undertaken via email or telephone.

Table 49: Choice of communication channel

\begin{tabular}{|l|l|l|}
\hline Channel & $\begin{array}{l}\text { Total } \\
\text { Interactions }\end{array}$ & $\begin{array}{l}\text { Individual } \\
\text { Count }\end{array}$ \\
\hline Face-to-face meetings & 28 & 12 \\
\hline Telephone & 10 & 5 \\
\hline Email & 25 & 13 \\
\hline Web-based technology(i.e. shared workspace, electronic collaborationtool) & 0 & 0 \\
\hline Other & 0 & 0 \\
\hline
\end{tabular}

Sixty percent of participants consider that face-to-face communication is an important factor in developing good working relationships. Given this preference, it is likely that participants might experience difficulties in building relationships with other participants, when there are issues of geographic distribution to contend with. 
The majority of communication, regardless of location, occurs less than once a week. Participants note that, other than for those who are co-located (i.e. the core team, and those located in the same organisations), geographic limitations inhibit spontaneous, or ad hoc, communication. This implies that when communication does occur, it is likely to be more formal in nature.

An individual's choice of communication channel is also influenced by the nature of the relationship with the recipient. The majority of participants indicate that, in general, the better they know an individual, the more likely that they are to communicate with them face-to-face (see Appendix 15); but seventy-nine percent also believe that it is difficult to build relationships and get to know others better without face-to-face communication. Several participants suggest that new relationships benefit from the ability to engage with an individual on a face-to-face basis. However, for established relationships, email can be a useful communication tool:

When you're trying to form a new relationship with somebody you want to make sure that they know you straight off the bat and get a fair idea of who you are...if it's a new relationship the best method for me is actually going and meeting them.

In addition, the choice of communication channel is influenced by the nature of the communication. This is seen as particularly important where knowledge is complex or where it is important to limit the possibility for misinterpretation:

I'd always prefer face-to-face [communication], but it depends on the issue.

If the topic's reasonably complex and there's differing views, I think email's counter-productive because people don't always express themselves well.

It's always good to be able to pinpoint what was said.

This suggests that, in this Case, a form of written communication, such as email, is more likely to be used in relation to complex knowledge, or where a written record of an interaction is required. 
Finally, the selection of communication channel is also influenced by the intention or purpose of the communication, and is considered particularly important if the purpose of the communication relates to change:

Face-to-face is probably the most important way we've found of actually changing people's perceptions.

We're requiring people to change the way they do business.

Several participants indicate that, in this Case, much of the work of the team focuses on facilitating change within the health sector. While much of this is dependent on the data itself, the way in which it is communicated can assist the change process. In this respect communication and sharing knowledge face-to-face is believed to significantly enhance the likelihood of change.

\subsection{The use of Information and Communication Technology}

In Case 4, ICT is used both as a communication tool, and as a means to store and analyse information. Participants' use of ICT in Case 4 is influenced by physical proximity, the availability of ICT tools, and the technical ability of individuals.

The main use of ICT in Case 4 is as a means of communication between members of the inter-organisational team. As detailed in the previous section, email is a key communication channel and is used to combat the lack of physical proximity between participants. It is also considered a helpful tool in ensuring that certain aspects of communication are formally recorded, as noted by a Health1 participant:

If I am seeking to get a very clear documentation of something that I'm about to escalate.

Survey responses indicate that participants are divided about whether email communication could help to build trust between team members (see Appendix 15), however individual interviews established that while email can help to build trust in existing relationships (i.e. where the basis of the relationship has already been formed through face to face contact), it is not useful for the initial establishment of relationships: 
I use a lot of email when I've already established the relationship but that's because they already know who I am.

Participants are also concerned about the opportunity for email communication to be misinterpreted, and the damage that could result from this:

It's an instrument that has to be used with a lot of care. Email is bereft of a lot of the subtle cues that are there in face-to-face communication.

ICT is also used to store and analysis the data relating to the collaboration. For the most part, this is managed at an organisational level, with each participating organisation storing its own data. There is no integration between the information systems used by each organisation, and the sharing of data is mainly co-ordinated through the emailing of data reports. Health2 also has a database that aggregates the collected data, but this is only available to Health2 participants and is not shared with other organisations participating in the Case:

We do have a database which generates all the reporting information and does what it needs to generate the reports.

Details of the Case can also be found on Health2's organisational website. There are plans to investigate the development of a website dedicated to the Case that all members and wider parties could access for simple queries. Advancing these plans is dependent on several factors including funding and a better awareness of the technical ability of prospective users. While most participants in the inter-organisational team are ICT savvy and are confident in using ICT tools, this is not the case for individuals in the wider health sector who often lack technical prowess. As two Health2 participants note:

If you're talking to a manager who doesn't even know how to use Excel very well, then giving them access to a set of data via the internet, or even through email can be quite a tedious process.

We send reports out by email and then we get phone calls asking if we can print them out...we've set up printing mechanisms for them because we know they want 
to print them out, even when they are 100 page reports, but they still ask us to do it for them.

The collaboration does not feature use of any e-government tools such as shared workspaces. Discussion with interview participants revealed that awareness of these tools is limited to Health1 participants who are aware of them but have had little direct experience in using workspaces.

Survey responses indicate that while forty percent of participants find the available technologies effective, forty percent are undecided, and twenty percent disagree (see Appendix 15). Fifty percent of participants indicate that there would be benefit to the collaboration from a wider range of ICT tools.

\subsection{Social Capital}

This section examines the aspects of Case 4 that relate to SC and how SC influences knowledge sharing in this Case.

\subsubsection{Relational social capital}

In Case 4, some participants place a higher value on aspects of relational SC than others. This is most evident in members of the team whose primary purpose is to manage and co-ordinate the day-to-day activities of the collaboration and who seem to have a greater awareness of these issues.

\section{Identity}

In Case 4, establishing the collaboration's identity within the wider health sector is regarded as more important than establishing a sense of identity among team members. It is perceived as an important aspect of the collaboration, and is one of a number of issues that the team leaders are dealing with:

We're still trying to establish our own identity amongst many other things that are happening.

The data indicate that members of the Core Health2 team place a great deal of importance on establishing a positive identity for the collaboration, and a number of 
actions have been taken to help establish this identity. For example, Health2 have established dedicated webpages to promote the collaboration and communicate its benefits. Members of the core team also spend much of their time travelling around the country to meet with organisations and attend health-related conferences. Details of the collaboration are also communicated through a national road show that was designed and delivered by the core team:

We actually went out and did a national road show that enabled us to find another way of getting people's attention.

This type of event raises awareness for the collaboration and confirms that establishing awareness of the venture within the wider health community is a higher priority than building relationships between team members. However, Jane believes that developing the identity of the venture is likely to contribute to participants' sense of belonging to the collaboration, and will help to establish a group identity:

It [identity] motivates you to be part of something. I think they all [participants] had a passion to be part of the project, which is always a bonus.

These comments suggests a link between identity and individual commitment; when group identity is strong, then an individual's commitment is likely to be higher.

Jane, who has worked in both Health1 and Health2, states that a feeling of belonging is easier to establish in less formal organisations.

At Health1, because there's so many level of process, you actually don't feel like you're a part of anything in particular. Whereas here, (Health2), I'm able to actually participant in the change and get some recognition where we need it on the change being successful.

Her comments reveal that being fully involved in a collaboration from its inception helps to build an individual's sense of commitment to, and identity with, the collaboration. In this way, participants are able to feel like they are making a difference. 
Trust

In Case 4, trust is an important motivator of knowledge sharing and is the second most significant factor in motivating individuals to share their knowledge with others (see Table $\mathbf{5 1}$ in the following section).

All participants consider trust to be an important aspect of collaboration, and indicate that lack of trust can be the most significant knowledge sharing barrier for interorganisational collaboration.

You can tell when someone has the same willingness to co-operate and trust and to me that's a keystone.

Assessing levels of trust within their own collaborative venture, forty-five percent of participants believe that trust levels are above average, however a further forty-five percent indicate a neutral response, and five percent believe trust levels are at or below average. Analysis of the distribution of neutral responses indicates that the majority of these responses are made by participants in three of the four support organisations (Support1, Support3, and Support4). This suggests that individuals in these organisations may experience less interaction with other participating organisations; thus it is difficult for them to assess to the levels of trust.

Trust building in this Case is related to individual competence, and this is perceived as something that develops over time and with experience:

It is based on proven results. The health sector is such a dynamic sector and it's forever evolving, but it also relies on past experiences within that specific area.

The geographic distribution of team members means that building trust between team members is a more difficult task than when individuals are co-located. Mechanisms that are considered useful in counteracting this barrier include demonstrating respect for other members and focussing on open and honest communication. This is a key concern for Alexa and Jane who hold the predominant responsibility for building relationships across the inter-organisational team: 
You have to treat them [participants] with respect, but I've learnt that through many years of being involved and watching relationships develop.

Most of my method [of working] is based on communication and that's how I build my trust with a lot of people.

At the outset of the collaboration, the formation of trust among inter-organisational team members was negatively impacted by issues at an organisational level:

One reason why people aren't open and honest up front is because a lot of people are dealing with the own environment and making sure they dot the I's and cross the T's.

Knowledge sharing in the health sector in some areas can be quite threatening because there's a business to protect or there's an intellectual property to protect and I think it is dependent on how well you form a safe environment for them, for them to feel comfortable.

This lack of trust was significant at the outset of the collaboration, and manifested itself in various ways including resistance to sharing information as well as issues relating to governance of the collaboration, and the need to maintain overall control of the data. As a Health2 participant notes:

They [Health1] were concerned about losing control of the data. I think we [Health2] actually demonstrated that we were working in a transparent manner, our system and things were strong and robust and they had nothing to worry about. Increasingly the angst around control has reduced and reduced and reduced from their perspective.

Data gathered from discussions with case participants indicates a symbiotic relationship between trust and the leadership and decision-making within the collaboration. When Health1 experienced a perceived lack of trust from Health2, they increased their focus on formal process. In turn, this led to further issues of trust between the organisations. Over the last year, the impact of organisational issues has lessened, due in most part to changes of personnel at both Health1 and Health2. These changes led to better 
documentation of processes and increased levels of communication across the collaboration which has resulted in greater openness within the inter-organisational team and an increase in the level of trust between the two lead organisations.

Norms, Obligations, and Expectations

In Case 4, the establishment of norms has been influenced by the power struggles between the two key organisations in the Case (Health1 and Health2).

The previous section identified that, at the outset of the collaboration, the group experienced difficulties related to trust and control between the two organisations who have overall responsibility for this Case (Health1 and Health2). These difficulties also impacted the establishment of norms within the inter-organisational team. Coleman (1990) defines norms as consensus based, and that reflect the values of the group or community. However, in this Case, the evidence indicates that initial power tussles saw Health1 attempting to impose levels of control over the group that inhibited the natural development of norms within the team. To a great extent, this appears to be a result of the different operational styles of the two organisations. Health1 operates on a formal basis and is guided by an established hierarchical structure and formal processes. Health2 is less concerned with formal processes and procedures and operates with a flatter, less hierarchical approach:

The bureaucracy of what happens at Health1...the different levels of sign-off that work has to go through. At Healthl, it's a more open environment.

These organisational differences meant that, at first, there was little opportunity for the team to move toward a consensus about the way in which the collaboration would operate:

There was quite a lot of power play between who would have more responsibility, Health1 or Health2.

However, as noted above, this situation changed with the appointment of new members to both the Health1 and Health2 teams. These changes heralded a new style of 
communication between the two organisations; one that was more open and was focused less on control and more on collaboration:

I think the clincher was when Healthl's formal responsibility and accountability was clearly and publicly vested in me...but it was actually the prior conversations that sort of prepared the way for that.

There's been change in the way the power has worked between the two organisations.

These changes have enabled the inter-organisational team to settle into a pattern of working that blends the needs of the two organisations. The initial norms imposed on the team have now become a blend of formal processes that guide work within the team and, to a lesser extent, norms that are established through discussion with the team. In addition to supporting the group to establish its own norms, these changes have also assisted the development of trust and contributed to the establishment of an identity for the collaboration.

Commitment to the collaboration and its outcomes provides the main motivation for the sharing of knowledge between team members and is a key facet in the development of individuals' expectations of others within the inter-organisational team. This commitment appears to be primarily driven by participants' collective belief and dedication to health outcomes. The collaboration is viewed by team members as an important step in improving health outcomes in New Zealand, and this contributes to the expectation that each participant will play their part. This is evidenced by the number of team members who indicate that they have been engaged in the health sector in a variety of roles in different organisations:

I've also spent at lot of time in other areas of the Ministry and health sector and I have built up a reputation of being able to deliver.

This comment suggests the expectation that participation in the collaboration is a reflection of an individual's reputation in the marketplace; the success of this endeavour can lead to further enhancement of this reputation. 


\subsubsection{Cognitive social capital}

The analysis of cognitive SC focuses on issues of shared language, shared purpose and goals, and a sense of shared culture.

\section{Shared language}

Shared language plays a role in Case 4 in respect to the development of sector-wide performance indicators; as a key mechanism by which knowledge can be shared to develop new and existing concepts; and finally as a tool to assist group cohesion and help build relationships.

A main focus of the inter-organisational team is the development of performance indicators that enable health data to be systematically analysed and interpreted regardless of how and where it is collected. In this respect, the performance indicators represent the development of a language or coding system that provides the sector with a consistent terminology and understanding in relation to measuring performance in the areas relating specifically to this Case. It also enables health professionals to understand the "big picture" in respect to the improvement of health outcomes for New Zealanders, and the way in which they, as individuals, contribute to these health outcomes.

Communication is considered a key aspect of the collaboration and there is a considerable emphasis on ensuring that communication is open, honest, and inclusive. This focus on communication is particularly important in this Case, where an initial lack of communication contributed to the difficulties experienced by the team in the early stages of the collaboration. This was particularly evident in regard to developing ways of working and determining who was responsible for making decisions within the collaboration.

When one starts unpicking one realises one is applying a different meaning to the same words and so forth. You know when you go quite deep into something and you think you've got a fundamental understanding and then something comes up that you realise actually you've meant different things and you aren't actually communicating in the way that you thought you were. 
Establishing a stronger, more positive focus on communication has helped to alleviate some of these issues and has also opened the way for other organisations to play a greater role within the collaboration. Thus, a focus on language and communication supports the discussion of ideas among team members, and assists the level of cohesion between participants.

\section{Shared Purpose and Goals}

The findings from Case 4 indicate that individuals are clear about the purpose of the collaboration and are committed to helping this purpose to be achieved:

Regardless of what side of the fence we're both on, we have an honest and open relationship to ensure that the outcome of what we're trying to achieve is successful.

However, though fifty-five percent of participants indicate that they are able to balance the interests of their own organisation with the interests of the group, other participants indicate that organisational needs can sometimes impact on their participation in the Case, particularly in relation to sharing information with other participants:

It is quite difficult working across agencies because at the end of the day you generally are looking for the same outcome but you may have been told by your master to do something in a different way to keep some information back from this group.

Dedication to the goals and outcomes of the collaboration is also evidenced by participants' identification of their commitment to the collaboration as the primary factor that motivates them to share their knowledge with other Case participants (see Table 50). 
Table 50: Motivation to share knowledge

\begin{tabular}{|l|c|c|}
\hline Motivation & $\begin{array}{c}\text { Total } \\
\text { Count }\end{array}$ & \begin{tabular}{c} 
Individuals \\
\hline Trust in the individual
\end{tabular} \\
\hline The feeling I am able to help that person & 4 & 9 \\
\hline The belief that the recipient will share their knowledge with me in return & 7 & 5 \\
\hline Commitment to the collaboration & 34 & 10 \\
\hline Presence of technology that makes sharing easy & 0 & 0 \\
\hline Recognition of my own knowledge and expertise & 1 & 1 \\
\hline
\end{tabular}

Participants indicate that, at the outset of the collaboration, the sense of shared purpose was more problematic. Although there was a clearly stated objective to develop performance indicators, deciding how this would be achieved took some time to finalise and conceptualise in a manner that was clear:

There wasn't necessarily a consensus even though we tried to build a consensus around indicators...it was difficult and people had their own view.

If you have to get to a common objective there probably will be some resistance and that's okay, that's just the way things happen.

To a large extent, initial difficulties associated with achieving "buy-in" to the shared purpose were due to organisational level issues that impacted on the ability of the group to develop a sense of shared purpose across the inter-organisational team, particularly in relation to the two main contributors, Health1 and Health2:

Health1 is a large organisation and from time to time there are competing objectives and there are people who have ingrained views of how things should work and they bring that to the table.

As has been previously detailed, tensions between the two organisations manifested primarily through control and governance issues between Health1 and Health2. This was due to the very different cultural styles of the two organisations (see the following section), and the way in which these differences manifested within the interorganisational team. These difficulties impacted on the ability to agree on the fundamental issue of shared purpose, and it was not until these issues were resolved that the shared purpose was clearly articulated. 


\section{Shared Culture}

Similarly to other aspects of this Case, the culture of the group appears to have changed over time. During the early stages of the collaboration, the establishment of a sense of shared culture within the inter-organisational team was inhibited by previously outlined issues between Health1 and Health2.

The majority of participants believe that difficulties can arise when organisations with different cultures come together for collaboration purposes, and this can have a negative impact on participants' ability and propensity to share information and knowledge with other Case participants.

Sixty percent of Case participants consider the culture of the inter-organisational team to be different to the culture within their own individual organisations (see Appendix 15). Further analysis of this result indicates that this belief is spread across the participating organisations. This suggests that, despite Health1 and Health2 playing more central roles within the Case, neither of these organisations' cultures significantly influences the culture of the inter-organisational team. This may be due to the very different cultures of the two organisations. Participants from Health2 note that:

The size of the organisation [Health1], and probably the diversity and silo [thinking] makes it difficult to achieve something that by its nature is cross cutting and requires collaboration.

It's [Health1] is very different from the culture here [Health2]. We don't have the multiple levels of hierarchy in terms of management...it's a very open environment; the job gets done a lot quicker than it does at [Health1].

These differences in culture are related to the very distinct roles of Health1 and Health2 which require different approaches to collaboration. For example, Health1 plays a lead role within the health sector and is responsible, in consultation with others, for the development of New Zealand's health-related policy. In this respect, Health1 holds a strong mandate within the sector. By contrast, Health2 was formed as a body to support and co-ordinate strategic activity of a group of health organisations within the sector. As such, their mandate is dependent on collaboration and collective activity. 


\subsubsection{Structural social capital}

Structural SC focuses on both the formal collaboration structure (who reports to whom) and the informal structure (who interacts with whom). The formal structure of this Case is detailed in Section 7.1. The collaboration is characterised by a lack of formal process and procedure, but rather is reliant on the experience and expertise of individuals to deliver to the collaboration goals. Despite the involvement of individuals with different levels of experience and at different steps on their respective hierarchical ladders, there is no formal reporting hierarchy within the collaboration, other than to the project leader. These results indicate that, overall, the collaboration operates on a relatively informal basis.

The informal structure was explored using SNA and is presented in Figure 30. This SNA data gathered in this Case portrays a highly centralised network that is characterised by weak ties. Participants of each organisation are positioned closely to other colleagues within the organisation and ties are present between each of the individuals. This suggests that organisational identity plays a role in determining ties within the network. The majority of relationships are uni-directional and there is a low level of reciprocity apparent within the network. Interactions are infrequent and most often take place less than once a week. Paul provides the single cutpoint within the network, and divides the network into two blocks, which sees Susan isolated from other network actors. There are 22 cliques evident with the network. 


\begin{tabular}{|llll|}
\hline & Health1 & Unreciprocated tie \\
Reciprocated tie \\
0 & Health2 & & \\
\hline & Support1 & & \\
\hline & Support2 & & \\
0 & Support3 & & \\
0 & Health3 & & \\
\hline & Health4 & & \\
\hline
\end{tabular}

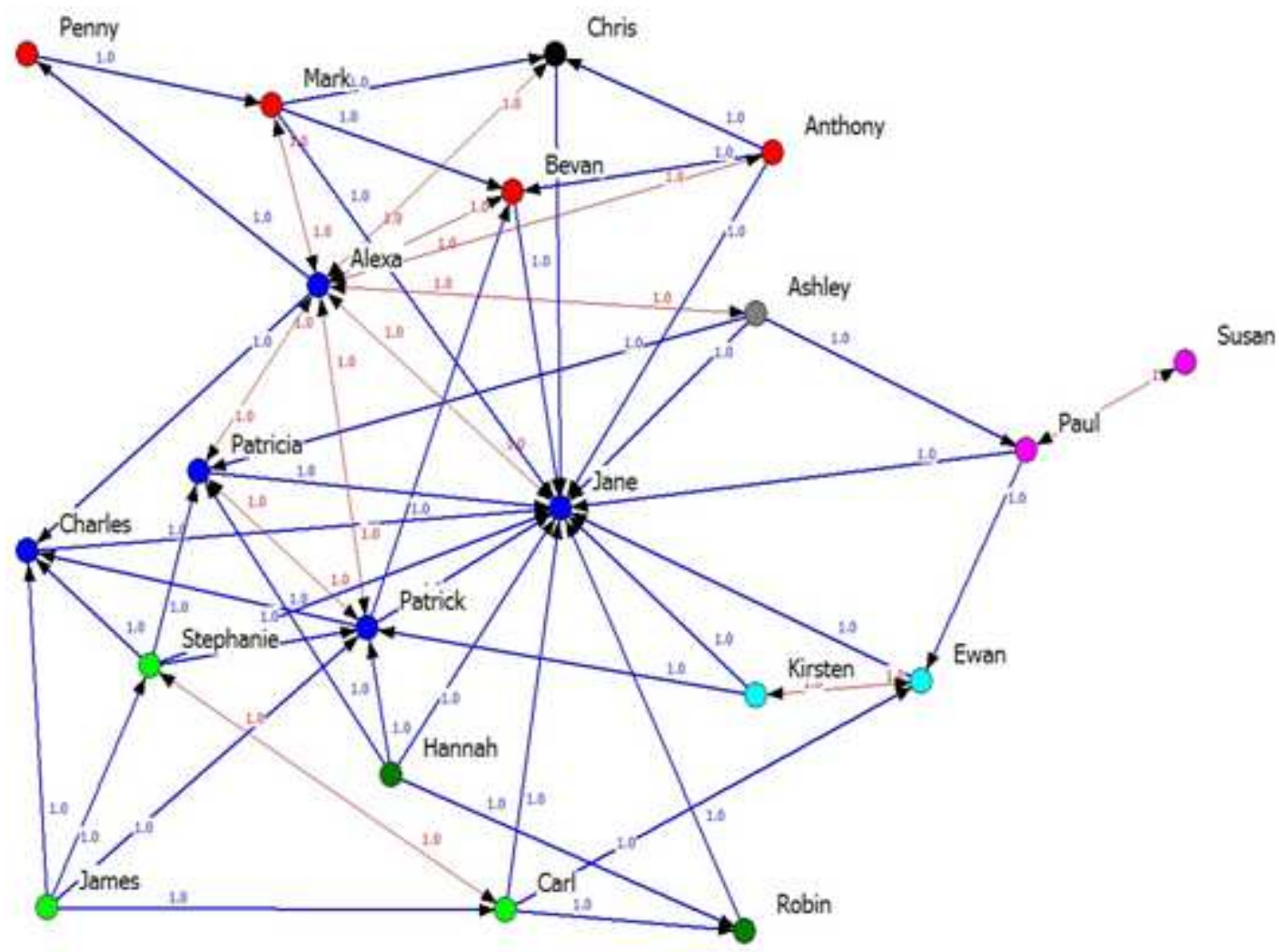

Figure 30: Informal network structure

Findings at both the network and individual levels are presented in the following sections.

\subsubsection{Network level results}

The network data gathered in Case 4 include the size, density, and distance measures as shown in Table 51. (Extended descriptions of these measures can be found in Chapter Three, p 96.) 
Table 51: Case 4 Network level measures

\begin{tabular}{|l|l|}
\hline Measure & Result \\
\hline Size & 20 \\
\hline Total ties present & 62.0000 \\
\hline Density (Mean) & 0.1632 \\
\hline Distance & 2.520 \\
\hline Std Deviation & 0.374 \\
\hline Variance & 0.140 \\
\hline Coefficient of variation (std dev/mean*100) & 222.619 \\
\hline
\end{tabular}

Case 4 represents the largest number of participants, and the greatest geographic distance of the cases in this research. The measures presented in Table $\mathbf{5 1}$ indicate a lack of cohesion in the knowledge sharing network. Both the network density (16\%) and distance measures (2.520) suggest that information moves slowly across the network, and that is it more difficult for participants to source information when they do not have a direct tie with another. The network density shows that there are low levels of connections among team members with only 62 of the total possible ties (380) in place across the network. Although a fully connected network would be highly unlikely and counter-productive to the flow of information in the network, the level of ties evident in Case 4 confirms the distributed nature of the network and the lack of interaction between some members of it.

\subsubsection{Individual level results}

Degree, closeness and betweenness centrality measures were selected to analyse the ties between individuals in the network. (Full descriptions of these measures can be found in Chapter 3, Section 3.3.4.2.).

\section{Degree Centrality}

Degree centrality measures the number of direct connections for each actor. A full list of normalised degree centrality degree ${ }^{19}$ scores for Case 4 is shown in Table $\mathbf{5 2}$.

\footnotetext{
19 Normalised scores divides simple degree by the maximum degree possible and enable the cross analysis of networks of different sizes.
} 
Table 52: Case 4 Degree Centrality Measures

\begin{tabular}{|c|c|c|c|c|c|c|}
\hline $\begin{array}{l}\text { Degree } \\
\text { Centrality }\end{array}$ & Actor & Formal Role & Organisation & NrmOutDeg & NrmInDeg & NrmDegree \\
\hline \multirow[t]{19}{*}{+} & Jane & Programme mgr & Health2 & 5.263 & 84.211 & 84.211 \\
\hline & Alexa & Programme $\mathrm{mgr}$ & Health2 & 52.632 & 42.105 & 52.632 \\
\hline & Patrick & member & Health2 & 26.316 & 31.579 & 47.368 \\
\hline & Patricia & member & Health2 & 15.789 & 26.316 & 31.579 \\
\hline & Stephanie & member & Support3 & 26.316 & 10.526 & 31.579 \\
\hline & Bevan & member & Health1 & 10.526 & 21.053 & 26.316 \\
\hline & Carl & member & Support3 & 21.053 & 10.526 & 26.316 \\
\hline & Charles & member & Health2 & 5.263 & 21.053 & 26.316 \\
\hline & Mark & member & Health1 & 21.053 & 10.526 & 26.316 \\
\hline & Anthony & sponsor & Health1 & 21.053 & 5.263 & 21.053 \\
\hline & Ashley & member & Health4 & 21.053 & 5.263 & 21.053 \\
\hline & Chris & member & Health3 & 10.526 & 15.789 & 21.053 \\
\hline & Ewan & member & Support4 & 10.526 & 15.789 & 21.053 \\
\hline & Hannah & member & Support2 & 21.053 & 0 & 21.053 \\
\hline & James & member & Support3 & 21.053 & 0 & 21.053 \\
\hline & Paul & member & Support1 & 15.789 & 10.526 & 21.053 \\
\hline & Kirsten & member & Support4 & 15.789 & 5.263 & 15.789 \\
\hline & Robin & member & Support2 & 5.263 & 10.526 & 15.789 \\
\hline & Penny & member & Health1 & 5.263 & 5.263 & 10.526 \\
\hline . & Susan & member & Support1 & 5.263 & 5.263 & 5.263 \\
\hline
\end{tabular}

The NrmDegree measures represent the total degree centrality of each actor, irrespective of the level of incoming and outgoing requests. This measure shows a large variance between the highest and lowest scores of actors. This can be seen in Figure 30, where Jane is positioned at the centre of the network and is frequently sought out for information and advice; whereas Susan is positioned at the periphery of the network. Without the tie to Paul, Susan would be completely isolated within the network.

Jane's high NrmDegree score reflects her formal role within the network where she is responsible for the day-to-day operations of the network. This requires Jane to interact with actors across the network to a greater extent than is required by other actors. This interaction enables her to be aware of the workings of the collaboration on a day-to-day basis. As evidenced by the qualitative data, Jane relies on frequent communication, particularly face-to-face communication where possible, to develop and maintain her relationships with individuals. Both the qualitative and quantitative data support Jane's view that communication is a key aspect of collaboration and is an important feature of her role. However, analysis of Jane's in-degree and out-degree scores show that while almost all members of the network seek her out for collaboration-related information and advice, Jane seeks advice from only one other individual in the network. The high 
level of demand on Jane indicates that she is perceived as a key knowledge source within the collaboration, but may also indicate that she is overloaded by the extent of the requests that are made of her, and this may also lead to disruptions or bottlenecks within the network

The NrmDegree measures also highlight the responsibility that Health2 members play in the day-to-day operations of the collaboration, particularly in relation to the four core members who are based in Wellington. It is interesting to note that the Auckland based member of Health2 is the only participant from this organisation to receive a lower NrmDegree score. This suggests that co-location of participants is more likely to influence knowledge sharing than organisational membership, and that participants are less likely to seek information from participants outside the core team.

Analysis of each actor's in-degree and out-degree measures reveals other interesting findings in relation to both individuals and organisations. The in-degree scores for Hannah and Robin (Support1) suggest that this organisation may play a less significant role in the collaboration than other support organisations, which enjoy better ties to a wider number of individuals. Indeed, the support organisations have limited interaction with each other, and their interaction with Health1 is dependent on members of Health2 to provide connecting ties. This confirms the qualitative evidence that showed the changing nature of the roles of Health1 and Health2 where the day-to-day management of the programme rests firmly with Health2, and confirms that initial governance issues have been resolved.

Network centralisation was also measured as an additional point of reference for the social network data. Network centralisation measures the global or macro level centralisation of the network and is indicative of how unequal the distribution of centrality is in a network, or how much variance there is in the distribution of centrality in a network. In this Case, network centralisation was derived from the the in-degree and out-degree measures shown in Table 53.

Table 53: Network centralisation index
\begin{tabular}{|l|l|}
\hline Centralisation Index & Result \\
\hline In-degree & $70.914 \%$ \\
\hline Out-degree & $37.637 \%$ \\
\hline Total degree centralisation & $63.1 \%$ \\
\hline
\end{tabular}


The overall degree centralisation index $(63.1 \%)$ indicates that activity in the network is highly centralised around a subset of individuals. This is evident in the sociogram (see Figure 30) where the majority of activity in the network is focused on Jane.

The centralisation measures also show a large degree of variance between in-degree and out-degree centralisation. This suggests that the majority of information requests are made of a very limited number of individuals; whereas the spread of requests (i.e. individuals seeking information) is less centralised indicating that many members of the network seek information. The high level of in-degree centralisation is significant and results in some individuals, such as Jane, occupying significant positional advantages in the network.

\section{Closeness centrality}

In Case 4, closeness centrality (see Table 54) is dominated by Health2 actors, and indicates that in addition to being the informational hub of the network, Health2 actors are also better positioned than other participants to seek information outside their direct sphere of influence. The analysis of closeness scores also suggests that support organisations are less influential than other organisations within the Case, due to the distance that separates them from other actors. 
Table 54: Case 4 closeness centrality measures

\begin{tabular}{|l|l|l|l|r|}
\hline $\begin{array}{c}\text { Closeness } \\
\text { Centrality }\end{array}$ & Actor & Role & Organisation & $\begin{array}{l}\text { Total } \\
\text { Closeness }\end{array}$ \\
\hline+ & Jane & Programme Mgr & Health2 & 100.438 \\
\cline { 2 - 5 } & Alexa & Programme Mgr & Health2 & 76.864 \\
\cline { 2 - 5 } & Patrick & member & Health2 & 64.504 \\
\cline { 2 - 5 } & Patricia & member & Health2 & 61.786 \\
\cline { 2 - 5 } & Bevan & member & Health1 & 60.52 \\
\hline & Charles & member & Health2 & 60.425 \\
\cline { 2 - 5 } & Chris & member & Health3 & 55.483 \\
\cline { 2 - 5 } & Mark & member & Health1 & 54.82 \\
\cline { 2 - 5 } & Ashley & member & Health4 & 53.976 \\
\cline { 2 - 5 } & Anthony & Sponsor & Health1 & 53.17 \\
\cline { 2 - 5 } & Penny & member & Health1 & 52.777 \\
\cline { 2 - 5 } & Paul & member & Support1 & 45.327 \\
\cline { 2 - 5 } & Ewan & member & Support4 & 44.314 \\
\cline { 2 - 5 } & Kirsten & member & Support4 & 37.824 \\
\cline { 2 - 5 } & Susan & member & Support1 & 36.976 \\
\cline { 2 - 5 } & James & member & Support3 & 35.159 \\
\cline { 2 - 5 } & Stephanie & member & Support3 & 29.306 \\
\cline { 2 - 5 } & Carl & member & Support3 & 29.013 \\
\cline { 2 - 5 } & Hannah & member & Support2 & 23.095 \\
\cline { 2 - 5 }- & Robin & member & Support2 & 20.824 \\
\hline
\end{tabular}

\section{Betweenness centrality}

The betweenness measures (see Table 55) show a large variance in the strategic positioning of individuals within the network, as shown by the overall variance measure of 152.408. These measures also confirm that Alexa and Jane, who occupy the leadership roles within Health2, occupy the most significant positions in the network and hold a high degree of power in relation to how information and knowledge are shared across the network. The betweenness scores take on further significance when one considers the variance between Jane's in-degree and out-degree scores which suggest her role as a gatekeeper within the network. Therefore, both Jane and Alexa enjoy significant positional advantages that enable them to apply some degree of control over the flow of information in the network should they so choose. 
Table 55: Case 4 betweenness centrality measures

\begin{tabular}{|c|c|c|c|c|}
\hline $\begin{array}{l}\text { Betweenness } \\
\text { Centrality }\end{array}$ & Actor & Formal Role & Organisation & Betweenness n \\
\hline \multirow[t]{20}{*}{+} & Alexa & Programme mgr & Health2 & 179.5 \\
\hline & Jane & Programme mgr & Health2 & 74 \\
\hline & Ashley & member & Health4 & 58 \\
\hline & Paul & member & Support1 & 57 \\
\hline & Patrick & member & Health2 & 28.75 \\
\hline & Ewan & member & Support4 & 19 \\
\hline & Mark & member & Health1 & 13 \\
\hline & Patricia & member & Health2 & 6.5 \\
\hline & Carl & member & Support3 & 6.25 \\
\hline & Stephanie & member & Support3 & 4.25 \\
\hline & Kirsten & member & Support4 & 3.5 \\
\hline & Charles & member & Health2 & 0.25 \\
\hline & Anthony & sponsor & Health1 & 0 \\
\hline & Bevan & member & Health1 & 0 \\
\hline & Chris & member & Health3 & 0 \\
\hline & Hannah & member & Support2 & 0 \\
\hline & James & member & Support3 & 0 \\
\hline & Penny & member & Health1 & 0 \\
\hline & Robin & member & Support2 & 0 \\
\hline & Susan & member & Support1 & 0 \\
\hline
\end{tabular}

At the other end of the scale, several individuals are at a significant positional disadvantage within the network. As Figure 30 shows, these individuals are located at the peripheries of the network and are highly reliant on other actors for information and knowledge. This might be of particular importance to organisations such as Support2, where both members of the team record zero betweenness scores.

\section{Cliques}

Analysis of the Case 4 network identifies 22 cliques, as shown in Table 56. This high level of cliques indicates the level of disconnection across the overall network, and is consistent with the low network density measure (16.32\%). 
Table 56: Case 4 cliques

\begin{tabular}{|l|l|l|l|l|}
\hline Clique & \multicolumn{5}{|c|}{ Actor } \\
\hline Clique 1 & Alexa & Anthony & Bevan & Jane \\
\hline Clique 2 & Alexa & Bevan & Jane & Mark \\
\hline Clique 3 & Alexa & Bevan & Jane & Patrick \\
\hline Clique 4 & Alexa & Ashley & Jane & Patricia \\
\hline Clique 5 & Alexa & Charles & Jane & Patrick \\
\hline Clique 6 & Alexa & Anthony & Chris & Jane \\
\hline Clique 7 & Alexa & Chris & Jane & Mark \\
\hline Clique 8 & Alexa & Jane & Patricia & Patrick \\
\hline Clique 9 & Carl & Ewan & Jane & \\
\hline Clique 10 & Carl & Jane & Robin & \\
\hline Clique 11 & Carl & Jane & Stephanie & \\
\hline Clique 12 & Ewan & Jane & Kirsten & \\
\hline Clique 13 & Ewan & Jane & Paul & \\
\hline Clique 14 & Hannah & Jane & Patricia & Patrick \\
\hline Clique 15 & Hannah & Jane & Robin & \\
\hline Clique 16 & Jane & Kirsten & Patrick & \\
\hline Clique 17 & Ashley & Jane & Paul & \\
\hline Clique 18 & Jane & Patricia & Patrick & Stephanie \\
\hline Clique 19 & Charles & Jane & Patrick & Stephanie \\
\hline Clique 20 & Charles & James & Patrick & Stephanie \\
\hline Clique 21 & Carl & James & Stephanie & \\
\hline Clique 22 & Alexa & Mark & Penny & \\
\hline
\end{tabular}

Twelve of the 22 cliques comprise four actors, the remaining cliques identify as triads (groups of three actors). Three is the minimum number of actors required for a clique. As these results show, there is an extensive degree of overlap between several of the cliques. Alexa (9) and Jane (8) belong to the most cliques and, as described above, these actors are focal points of the network. Their extensive clique membership is evidence of the level of informal networking they undertake, and is facilitated by their frequent travel to other Case organisations. The overlapping nature of their clique membership helps to diffuse information and knowledge through the network.

By comparison, Susan is not a member of any clique, and is the only actor in the network who is completely isolated. Susan's isolation is evidenced by the analysis of cut and blockpoints within the network. These measures identify the Case network's weakest areas, and indicate that should Paul be removed from the network, Susan would be totally disconnected from other actors within the network. 


\section{Tie Strength}

Case 4 is characterised by weak ties showing that, in general, interaction is infrequent and largely unreciprocated. The overall measure of reciprocity for the network is $19 \%$, indicating that less than one-fifth of the team enjoys reciprocal ties. This is consistent with earlier qualitative findings that suggest that Case 4 is, to a large extent, an information-based Case. As such, many of the interactions consist of data being shared with the central team - and these are likely to be largely one-way transactions - and there is limited need for interactions between organisations, other than between Health1 and Health2. Accordingly, ties between Health3, Health4 and the support organisations are limited with interactions more commonly directed at Health2 members.

The strongest ties in the network exist between individuals from the same organisation. Alexa, as one of the two programme managers, also enjoys strong ties with participants from Health1. This is reflective of her strategic role and, though she has lower centrality scores than Jane, the reciprocal nature of her relationships is likely to afford her a significant degree of influence across the network.

\subsection{Summary of Chapter 7}

This chapter has presented the key results and analysis of Case 4 of this research. The first section of this chapter presents the findings related to ICT within the Case. The chapter continues by presenting the findings related to SC and specific aspects of relational, cognitive, and structural SC that have an influence within the Case. The chapter also shows the informal network evident within the Case and presents both visual and quantitative measures to describe the configuration of the network and roles of individuals within the network.

This Case encompasses 20 participants across a range of health sector organisations. Initially, the collaboration experienced difficulties due to the relationship between the two main organisations in the Case, Health1 and Health2. These issues were largely due to the different cultures and operational approaches of the two organisations.

A focus on building the identity of the collaborative endeavour, and facilitating communication and trust across the inter-organisational team has enabled the team to 
operate more effectively. A combination of formalised processes and better communication has provided the group with greater autonomy to work together towards a shared purpose and to establish its own set of norms.

In this Case, ICT is used mostly for communication purposes. At an organisational level, ICT is also used for information analysis and storage, but there is no integration between disparate organisational systems. The nature of the Case is such that the information is relevant to a diverse range of individuals and groups within the sector, with a correspondingly diverse range of technical ability. This has inhibited the use of ICT and fostered the reliance on human-based interaction. 


\section{CROSS CASE ANALYSIS}

This chapter presents and discusses the key findings derived from the cross case analysis of the four Cases in this study. Systematic analysis was applied across the Cases to identify patterns of similarity and difference and, where possible, to determine the underlying factors contributing to these patterns. Understanding these patterns provides further insight into participants' knowledge sharing behaviours and the main themes of the study, and will enable the researcher to build a comprehensive picture of knowledge sharing in public sector inter-organisational collaboration.

The analysis is presented using the same structure previously used for the individual case studies. This enables a methodical pathway through the results that will identify the main aspects of interest of the study.

\subsection{Perceptions of knowledge}

As established in the literature review (see page 19), knowledge has been defined and interpreted from different perspectives. The purpose of asking Case participants to define knowledge, using their own words, was to stimulate their thinking and consideration of knowledge, and to encapsulate this thinking within their own knowledge definition. This enabled the researcher to better understand what knowledge means to individuals, the ways in which it manifests and is used by individuals, and to identify patterns that may contribute to participants' perceptions of knowledge. Discussions regarding knowledge and its meaning were also conducted throughout the interview process, and these deeper discussions helped to add clarity and understanding to the range of definitions proposed by participants.

Participants' definitions were amalgamated into a single meta-matrix through which textual analysis was undertaken using several characteristics derived from the literature, specifically from the aggregated terms proffered by Galliers and Newell (2001, see Chapter 2, p. 20). The meta-matrix is included in Appendix 8. Four inter-relating factors were most commonly associated with how participants perceive knowledge: (1) action, context, understanding, and experience. 
Firstly, several participants recognise a relationship between information and knowledge, where knowledge is perceived as the product or result of information that is used or applied in a situation. The focus here is on the "action" that is taken in relation to the information, and denotes an ability to move forward in a situation either through attaining understanding or by using the knowledge to enable and support decisionmaking. A Case 3 participant states that:

The ability to transfer data into information relevant for decision and the ability to make decisions that guide future actions, using learning and experience.

This aspect is closely related to the second factor of knowledge: context. Knowledge was commonly understood as contextual in nature. Thus, knowledge is context specific and relates to a particular situation or circumstance. In this study, participants perceive their own knowledge to be pertinent to the context of the inter-organisational collaboration in which they are engaged and, on a wider scale, to the sector or sub-set of the sector in which they are employed. For example:

In the context of this project, knowledge means contextual knowledge. So for example when something unexplained and odd pops up in the data someone with knowledge can tell you the reason for it, or the likely reason.

Thirdly, knowledge is also viewed as a manifestation of an individual's understanding of information, or a concept or situation. The individual's understanding was attained primarily through experience; thus understanding and the ability to provide insight into a situation is seen as the product of that experience. In practical terms, participants' experience within a particular field such as educational policy provides them with a deeper level of understanding of the Case, and enables them to apply that experience to Case-related information. The application of this experience and understanding provides insights that a less experienced individual may not bring to collaboration.

In addition to the four factors outlined above, the researcher also noted the presence of distinct knowledge types within the four Cases. This knowledge falls into two key categories: institutional and/or sector experience, and technical experience. In many instances, participants have been working with a particular organisation or within a 
specific area of the public sector for a significant period of their career. In these instances, participants have acquired a considerable amount of knowledge about the organisation and the specific sector (i.e. justice, health, education) in which they are employed. In other instances, individuals' experience is also related to a specific area of expertise, such as policy or research; thus they have built up a considerable wealth of technical expertise in their chosen field. Together, the accumulation of organisational, sectoral, and technical knowledge result in a considerable body of specialist experience and expertise.

The analysis found that knowledge is generally defined at the level of the individual, and is related to the accumulation of experience and expertise developed by an individual. Thus, knowledge is primarily perceived as a personal asset or characteristic that has been formed through one's experiences.

An individual's frame of reference is also relevant to participants' perceptions of knowledge. In particular, an individual's role and place in the hierarchy appears to influence their reflections on knowledge. Those in management positions are more likely to view knowledge as personalised (see Hansen et al., 1999) and consider that knowledge is closely tied to individuals' experience and understanding; while participants at the lower end of the hierarchical ladder identify knowledge as codified (see Hansen et al., 1999) and view it through the lens of documents and reports held in databases or other technology systems. The codification perspective is generally held by participants who are more commonly involved in operational-level tasks, and whose definitions tend to focus on systems, processes, and modelling as tools that could derive deeper meaning from information:

We [analysts] supply the data, the information. Then they [management] discuss it and ask questions. All we do is supply the raw facts.

By comparison, more senior participants working in management, policy, and research roles more commonly focus on factors such as learning, context, and understanding. Their perceptions of knowledge reflect learning that occurs through the synthesis of accumulated experience and expertise, and exposure to the knowledge of others. This suggests that access to, and use of, information and knowledge is linked with one's position within an organisation. For individuals occupying more junior roles, there is a 
stronger likelihood that they will deal with information; higher in the hierarchy it is more likely that one will demonstrate and be required to deal with knowledge. This finding also supports the link between knowledge and decision-making which is identified by twelve percent of the total participants, who link knowledge to the ability to make decisions, or define courses of action (see Appendix 8).

Social identity theory (SIT) can also been seen to play a role in understanding attitudes towards knowledge whereby an individual's personal identity within a group and/or parent organisation influences the way in which they perceive knowledge and their role in the utilisation of that knowledge. For example, in Case 1 the majority of participants identify themselves as dealing with information rather than knowledge, resulting in a collective identity as an information-based group. By contrast, participants in Case 3 consider their inclusion in the group to be an acknowledgement of the extent of the personal knowledge that they can bring to the collaboration.

Davenport (1998) states that within organisations, knowledge is closely tied to the individual, and as Galliers and Newell (2001) assert it is only when dealing with knowledge that individuals are required to take action. Therefore, for Case 1 participants, their collective identification as an information-based group relieves individuals of the need to take action or make decisions (other than at a leadership level) and reduces the level of risk can be directly associated with them as individuals.

\subsection{Motivation to share}

Motivation to share knowledge is related to several aspects of the research. In the individual Cases, motivation was discussed within the sections relating to relational SC. In this chapter, motivation is discussed as an aspect in its own right. It is closely related to issues of SC and also has relevance for the ICT findings and will be referred to within each of these sections. However, for clarity purposes, the main results pertaining to motivation are presented here.

The literature suggests that motivating staff to share knowledge is a key consideration, and Edwards et al. (2003) identify this as one of the most important challenges for organisations. 
In this research, individuals' motivation to share knowledge with other Case participants was explored through the survey. A range of motivating factors were drawn from the knowledge sharing literature and participants were asked to identify, for each of the person with whom they shared knowledge, what the key motivational factor was in each case. Participants were also given the opportunity to provide an individual response if none of the options were appropriate. Analysis of participants' responses reveals that an individual's motivation to share varies according to whom an individual is sharing with.

Factors that motivate individuals to share knowledge include trust in the other individual and a feeling of being able to help the other individual. An expectation of reciprocal sharing was most prominent $(31 \%)$ in Case 1, although this primarily relates to individuals within the same organisation. The expectation of reciprocity also denotes the presence of a power-play between individuals. The initial sharer of knowledge places the recipient in a position where they may feel obliged to reciprocate, placing the initiator in a position of power. Conversely, non-reciprocation places the recipient in the power position by choosing not to reciprocate.

Analysis indicates that, in each Case, an individual's commitment to the collaboration provides the predominant motivation to share knowledge with other team members (see Table 57). In total, fifty-one percent of participants' sharing behaviours were motivated by their commitment to the collaboration.

\begin{tabular}{|c|c|c|c|c|c|}
\hline Motivation & ণ্ & $\begin{array}{l}N \\
\tilde{y} \\
\tilde{U} \\
\tilde{U}\end{array}$ & 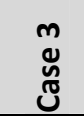 & 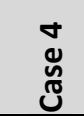 & $\stackrel{\bar{\pi}}{\stackrel{0}{\circ}}$ \\
\hline Trust in the individual & $10 \%$ & $11 \%$ & $23 \%$ & $24 \%$ & $17 \%$ \\
\hline The feeling I am able to help that person & $12 \%$ & $33 \%$ & $0 \%$ & $6 \%$ & $13 \%$ \\
\hline $\begin{array}{l}\text { The belief that the recipient will share their knowledge with } \\
\text { me in return }\end{array}$ & $31 \%$ & $15 \%$ & $10 \%$ & $8 \%$ & $16 \%$ \\
\hline Commitment to the collaboration & $36 \%$ & $41 \%$ & $68 \%$ & $58 \%$ & $51 \%$ \\
\hline Presence of technology that makes sharing easy & $0 \%$ & $0 \%$ & $0 \%$ & $0 \%$ & $0 \%$ \\
\hline Recognition of my knowledge and expertise & $12 \%$ & $0 \%$ & $0 \%$ & $3 \%$ & $4 \%$ \\
\hline
\end{tabular}

As illustrated in the individual Cases, commitment to a collaboration is not confined solely to the goals or outcomes of the collaboration. Discussion with participants 
indicate that this value is associated more with the "area of work" that is being undertaken, and represents a commitment to that work, rather than a commitment to the collaboration, or to the inter-organisational team. This is also demonstrated through the extensive length of tenure that participants have acquired within either a single organisations, or to a particular subset of the public sector. Thus, knowledge sharing is driven by participants' commitment to the intrinsic nature of the work they do, rather than the inter-organisational collaboration which is commonly regarded as a vehicle through which the work is undertaken.

This finding is supported by the analysis that shows that even in Cases such as Case 2, where individuals voice concerns and frustrations with many operational facets of the Case, they still record a relatively strong level of commitment (41\%) - an unexpected finding. However, as documented within Case 2, the majority of this frustration relates to the way in which the collaboration operates, rather than to the value of the collaboration goals. Thus, it is surmised that when the purpose of the collaborative endeavour or area of work is seen to be of high importance or where the associated outcome/s have an impact on the wider population, then participants feel a strong sense of individual commitment due to their own beliefs and values, which motivates them to participate and share.

This research also identified an association between the perceived intrinsic value of the work and aspects of SC, for example a sense of shared identity, trust, or shared vision. This association can act to both enhance the collaboration (as in Case 3) or overcome issues apparent within the collaboration (as in Case 2). In Case 3, participants demonstrate a strong sense of commitment to the intrinsic value of the work, but there is also alignment and unity among participants in relation to the overall goals of the collaboration and a strong sense of group identity. Individuals are highly motivated to share and this is evidenced by the frequency of interactions and extent of reciprocal sharing as illustrated by the SNA data (detailed below). In Case 2, individuals' commitment acts as a motivator to overcome a lack of shared purpose, and to counteract issues of trust. In these circumstances, individuals use their own belief in the importance of the collaboration to put aside issues relating to barriers such as different agendas or lack of shared purpose. 
Meyer and Allan (1997) distinguish between commitment types and posit that affective commitment constitutes an individual's goal to remain working within an organisation, rather than being required to (continuance commitment), or being duty-bound to remain (normative commitment). Although Meyer and Allan focus on commitment at an organisational level, these commitment types are also relevant at an inter-organisational level. This research found many participants, through their length of organisational tenure and dedication to the goals of the organisation, demonstrate affective commitment at the organisational level. However, this research also suggests that affective commitment extends to both the sub-sector and sector level. This finding is based on discussions with participants that, in some instances, revealed discontent with the way in which their parent organisations or the sector function, but a strong sense of commitment to contribute to the outcome of the work of the organisation or the wider sector nevertheless. As a result, participants remained within their roles, or associated roles within the sector in order to continue to contribute to the field.

Kelloway and Barling (2000) posit that an employee's commitment to an organisation will positively affect individual performance, and will be manifested through a reciprocal relationship between the individual and the organisation. In this research, this relationship was explored at an inter-organisational level, through a comparison of the level of commitment reported by each Case, as well as the actual levels of reciprocity measured during the SNA (see Table 58).

Table 58: Comparison of commitment and reciprocity levels

\begin{tabular}{|l|r|r|}
\hline Case & Reciprocity & Commitment \\
\hline Case 1 & $37.5 \%$ & $36 \%$ \\
\hline Case 2 & $32 \%$ & $41 \%$ \\
\hline Case 3 & $42 \%$ & $68 \%$ \\
\hline Case 4 & $19 \%$ & $58 \%$ \\
\hline
\end{tabular}

This data shows that the highest level of reciprocity (42\%) was recorded in Case 3, which also reported the highest level of commitment (68\%). However, interpretation of the data becomes more complex when one considers that Case 4 participants also indicate a high commitment to the collaboration, but enjoy much lower levels of reciprocity. These results indicate that while commitment is a significant factor in determining an individual's motivation to share, it cannot be deemed the sole influence. 
Rather, as this research shows, a range of factors contribute to knowledge sharing at an inter-organisational level.

\subsubsection{Knowledge sharing activities}

A knowledge sharing activity is defined as a formal or informal opportunity through which knowledge can be shared between Case participants. Ipe (2003) identifies formal activities as purposively designed opportunities such as structured meetings and shared information repositories, whereas informal opportunities are more opportunistic in nature and are more likely to occur through face-to-face interaction.

The nature of the inter-organisational collaboration is itself an example of a "structured work team". It represents a team that has been purposively constructed to bring together a range of knowledgeable individuals to contribute to the achievement of a specific objective. In each Case, the inter-organisational team has been formally structured to enable information and knowledge from each of the participating organisations to be shared and utilised to achieve the goal of the collaboration. Within each Case, participants have access to a range of formal and informal activities through which knowledge can be shared.

The research found that the range of knowledge sharing activities available within each collaboration varies. Both formal and informal knowledge sharing activities are available to participants, but the extent to which these are available differs across the four Cases (see Table 59).

Table 59: Availability of knowledge sharing activities

\begin{tabular}{|c|c|c|c|c|}
\hline Knowledge Sharing Activity & Case1 & Case 2 & Case 3 & Case 4 \\
\hline Structured Meetings & & $\sqrt{ }$ & $\sqrt{ }$ & \\
\hline Written reports/documentation & J & $\sqrt{ }$ & J & $\sqrt{ }$ \\
\hline Shared Information Repositories & & $\sqrt{ }$ & J & \\
\hline Shared Workspaces & & J & J & \\
\hline Email & $\sqrt{J}$ & J & $\sqrt{ }$ & J \\
\hline Telephone & $\sqrt{ }$ & J & $\sqrt{ }$ & J \\
\hline Informal face-to-face activity & & & $\sqrt{ }$ & \\
\hline
\end{tabular}

Formal activities include structured meetings, shared information repositories, and sharing through formal documentation such as reports, discussion papers, and meeting notes. Informal activities, such as opportunistic face-to-face meetings are less common, 
and typically occurred only in Case 3, which represents the single instance of a colocated team. Indeed, Case 3 participants enjoy the greatest range of activities with access to both formal activities such as structured meetings, a shared information repository and shared workspace, and informal activities such as ad hoc face-to-face interaction. Although Case 2 participants have access to a similar range of activities, they have no ability for ad hoc interaction, thus the majority of sharing activities occur as formal interactions. This is also the situation for participants in Cases 1 and 4 who indicate a very limited range of both formal and informal knowledge sharing activities are available.

Across all four Cases, the majority of participants (61\%) signal that they frequently learn more from an informal chat than from a formal meeting, and that these informal activities, although rare, are highly valued. In Case 3, informal communication is the most common way for knowledge to be shared and is made possible by the close proximity of team members. Participants report that if they are seeking information or need to discuss an aspect of the collaboration, they are more likely to approach another team member for an informal discussion which would take place at the team member's desk. Participants indicate that, in some instances where the discussion may be lengthy, they might use a quiet space or meeting room, but this would be largely opportunistic and that no formal booking of a room would be made. In each of the other Cases, informal activities are largely confined to participants housed within the same organisation.

Participants, particularly in Cases 1, 2, and 3 also identify links to other groups such as working parties, or contacts within individuals' parent organisations from whom they were able to seek advice ${ }^{20}$. These groups provide connections to other sources of information and knowledge assets that are not available within the inter-organisational teams. Thus, individuals are able to individually access knowledge and ideas that they then bring back to the group and share. These links are also highly valued.

\footnotetext{
20 These individuals were specifically identified on the questionnaire completed by participants and through initial investigations with team leaders.
} 
As Table 59 shows, knowledge sharing through ICT tools focuses predominantly on the use of email and the telephone. Cases 2 and 3 also have access to shared drives and shared workspaces, although use of these tools is limited. (A full analysis of the use of ICT tools can be found in Section 8.3).

In addition to the availability of the activity, participants' choice of knowledge sharing activity is influenced by the physical proximity of participants, and the nature of the knowledge being shared. For example, where the majority of sharing is conducted through email and/or other technology tools, participants often relate this to information sharing. When participants require a greater depth of understanding about a document, or aspects of the collaboration, they are more likely to select face-to-face communication, through either formal or informal means.

\subsubsection{Choice of knowledge sharing channel}

Knowledge sharing activities can be facilitated through a range of communication channels. This research found that, even in distributed Cases, participants indicate that, whenever possible, they prefer to share knowledge through face-to-face interaction, whether that be through a formal opportunity, such as a structured meeting, or through an informal activity such as a casual, or opportunistic conversation.

In total, fifty-one percent of knowledge sharing takes place through face-to-face communication; thirty-nine percent via email; and nine percent by telephone (see Table 60). The highest level of face-to-face interaction (78\%) was recorded in Case 3, the only co-located collaboration.

Table 60: Use of knowledge sharing channels
\begin{tabular}{|l|r|r|r|r|r|}
\hline Channel & \multicolumn{1}{|c|}{ Case 1 } & \multicolumn{1}{c|}{ Case 2 } & \multicolumn{1}{c|}{ Case 3 } & \multicolumn{1}{c|}{ Case 4} & Total \\
\hline Face-to-face activity & $45.83 \%$ & $38.71 \%$ & $78.16 \%$ & $44.44 \%$ & $\mathbf{5 1 . 7 9 \%}$ \\
\hline Email & $39.58 \%$ & $54.84 \%$ & $21.84 \%$ & $39.68 \%$ & $\mathbf{3 8 . 9 9 \%}$ \\
\hline Telephone & $14.58 \%$ & $6.45 \%$ & $0.00 \%$ & $15.87 \%$ & $\mathbf{9 . 2 3 \%}$ \\
\hline $\begin{array}{l}\text { ICT collaboration tool (i.e. shared } \\
\text { workspace, discussion forum) }\end{array}$ & $0 \%$ & $0 \%$ & $0 \%$ & $0 \%$ & $\mathbf{0 . 0 0 \%}$ \\
\hline
\end{tabular}

The high level of face-to-face interaction was a surprising finding given the distributed nature of three of the four Cases. However, analysis of the survey and the social network data (see Chapters 4-7) shows that the majority of actual face-to-face 
interactions within the distributed Cases is between participants within the same organisation. For example, Case 1 involves only two organisations and the majority of interactions take place between participants within the same organisation.

Analysis of the factors related to a participant's choice of knowledge sharing activity indicates that the relationship with the recipient, the physical proximity of participants, and the nature of knowledge to be shared are the most influential factors in determining how knowledge sharing interactions occur (see Figure 31).

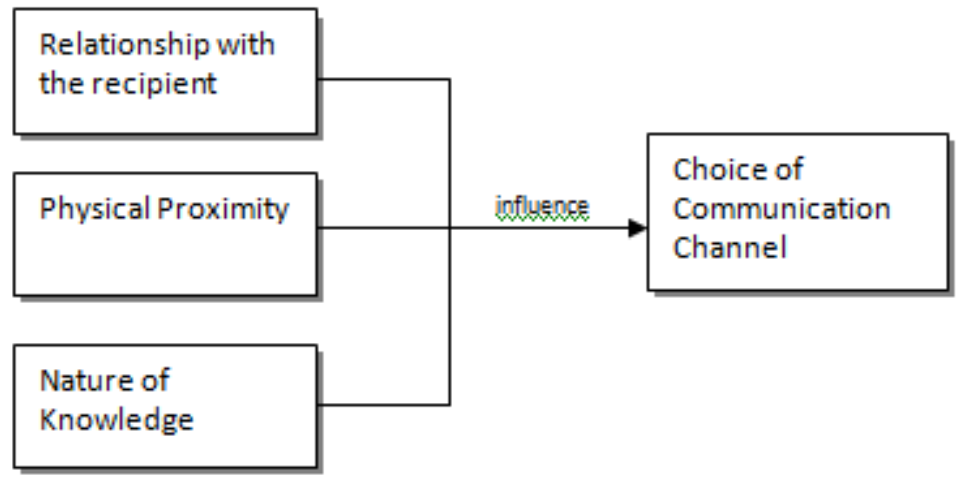

Figure 31: Factors influencing knowledge sharing activity

The relationship with the recipient was deduced by identifying if individuals had any form of relationship prior to the commencement of the inter-organisational collaboration; and whether, in their opinion, they are more likely to share with individuals whom they know better (irrespective of any prior relationship). A prior relationship was defined as having previously worked together, being part of the same business networks, or having a non-work-related relationship. The analysis revealed that very few individuals had prior relationships of any kind and that, other than other members from their own organisational team (i.e. where more than one participant from a team participates within the inter-organisational team), most individuals met for the first time on the collaboration. However, seventy-three percent of Case participants note that the more familiar they are with an individual, the more likely that they will share through some form of face-to-face interaction. Therefore, a focus on building relationships and increasing familiarity between individuals will facilitate knowledge sharing. As the rate of inter-organisational collaboration continues to rise (Walker, 2004), the level of familiarity between individuals may therefore occur naturally as it is likely that individuals will increasingly participate in inter-organisational collaborative 
ventures. Further, the specialised nature of the sectors within which they work means that it is likely that teams will often include the same individuals, thus familiarity among individuals will grow. This represents a significant opportunity for the development of a knowledge network across the sector and within sub-sets of the sector.

To a somewhat lesser extent, the nature of the knowledge also influences the choice of communication channel. Where the interaction concerns explicit knowledge, this is often facilitated through the use of an ICT tool. For example, Case 2 participants describe the way in which the change control system (held within the shared workspace) is used to vote on proposed changes to terminology. This finding is supported in Table 3 , above, which shows that no participants identify the change control system as a channel or activity through which knowledge is shared. This indicates that participants view the change control system as an "information" tool, and that where knowledge is to be shared about the proposed changes, this is facilitated through face-to-face activity, email, or telephone. Similarly, in Case 3, the use of a shared drive was found to be a good mechanism for storing and searching for Case-related documentation, but not for knowledge-based discussions. This supports Nonaka and Takeuchi's (1995) view that knowledge shared through formal channels such as ICT is likely to be largely explicit in nature, and thus facilitate knowledge that is more easily codifiable and can be more readily held within a storage mechanism (Lam \& Lambermont-Ford, 2000). In addition, where knowledge is complex in nature, or where the knowledge is viewed as having potential repercussions, individuals are more likely to select a communication channel such as email, which provides a documented audit trail.

The proximity of team members also influences an individual's choice of knowledge sharing channel. In Case 3, where participants are co-located, the greater part of sharing activity occurs through face-to-face interaction. However, in Cases 1, 2, and 4, and for interactions between Case 1 organisations, email is more likely to be used. The physical proximity of participants was also found to influence the frequency of interactions between individuals. Individuals who are co-located interact more than once a day. This frequency reduces to once a week for participants located in the same building, but on a different floor. For team members located in different buildings, or different cities, sharing is more likely to occur less than once a week (see Appendix 16). 
Other studies suggest that team members will be most strongly influenced by those with whom they have more frequent interactions (Epstein, 1961, Kadushin, 1966 in Lang 2004). Therefore, those individuals who have more frequent interaction with others are likely to be more influential within the collaboration. This is also evidenced in the Cases through the social network data which found that the most influential individuals in each Case occupy central positions in the informal knowledge sharing network and engage in frequent interactions with other individuals. In addition, these actions are often reciprocal, increasing the strength of the tie (relationship) between the two individuals.

\subsection{Facilitating knowledge sharing through ICT}

Analysis of ICT-related results across the four Case studies identifies five key findings. The first finding notes the limited availability of ICT tools to support information and knowledge sharing; the second finding indicates that the predominant uses of ICT relate to its use as a communication tool, and to a lesser extent as an information repository; the third finding confirms a low overall awareness of e-government initiatives; the fourth finding identifies that availability of ICT tools is largely a result of decisionmaking at a leadership level. Finally, the fifth finding is that use of ICT tools in interorganisational collaboration is influenced by participants' levels of technical ability; the physical proximity of participants; the nature of the knowledge to be shared; and the perceived ease of use of the tool.

\subsubsection{ICT availability}

While ICT provides participants in each Case with access to basic communication tools such as email and the telephone, more sophisticated collaborative tools such as shared storage systems and shared workspaces are available only to participants in Cases 2 and 3 (see Table 61). None of the Cases has access to weblogs, wikis, instant messaging, or 
social networking tools ${ }^{21}$. Despite the limited availability of ICT tools, only Case 4 participants indicate that the Case would benefit from a greater range of ICT tools.

Table 61: Availability of ICT tools

\begin{tabular}{|l|c|c|c|c|}
\hline ICT Tool & Case 1 & Case 2 & Case 3 & Case 4 \\
\hline Shared Information Repositories & & $\sqrt{ }$ & \\
\hline Shared Workspaces & & $\sqrt{ }$ & $\sqrt{ }$ & \\
\hline Email & $\sqrt{ }$ & $\sqrt{ }$ & $\sqrt{ }$ \\
\hline Telephone & $\sqrt{ }$ & $\sqrt{ }$ & $\sqrt{ }$ \\
\hline
\end{tabular}

\section{Information storage}

Sixty-three percent of all participants confirm that in their parent organisations there is a strong emphasis on facilitating information sharing through ICT tools (see Appendix 17). Eighty-six percent of participants agree that use of ICT tools makes information easier for individuals to find (see Appendix 17). Thus, at an organisational level, there is widespread use of ICT to store information, and make this available across the organisation. Given this emphasis, it is reasonable to anticipate that shared storage repositories would be available to each of the Cases. This assumption is supported by the fact that this research has already established that much of the sharing that takes place within the Cases focuses on explicit knowledge: that is, knowledge that is more easily codifiable. This is most evident in Cases 1 and 4 that, to a great extent, deal with the gathering and analysis of data and information. Equally, Case 2 focuses on making decisions relating to the sector's use of standard terminology, these decisions are often made based on the information provided and do not require the need for knowledge sharing between Case participants. However, as shown in the individual Case studies, at an inter-organisational level, shared information repositories are much less evident. In fact, only Cases 2 and 3 have access to a shared information repository. In all other Cases, information is stored within each participating organisation and was mainly shared through the use of email.

21 The e-Government website reports that in 2010, some inter-organisational project teams are now making use of weblogs, wikis, and online forums, but this was not evidenced during this research's data collection period of 2006/7. 


\section{Communication tools}

In each Case, ICT-related communication tools include both email and the telephone. As shown in Table 60 above, email use is highest in the three distributed Cases (Cases 1, 2, and 4). As illustrated within the individual case studies, email use is driven by the physical proximity of individuals which in many cases precludes the opportunity for face-to-face communication; the ability to provide a record of the interaction; and to enable individuals to move onto other tasks.

The study also found some evidence that email can, to some extent, assist with trust building. Overall, forty-one percent of the participants indicate that email can help to build trust within a relationship. Closer examination of these results shows that this belief is largely confined to participants in Cases 1 and 2. However, discussions during the interview process clarify that, while email may help to build trust in established relationships, it is less successful as an initiator of trust within new relationships; participants believe that face-to-face contact is required at some stage of the relationship in order for trust to be more fully established. Similarly, it was observed that while email can provide connections to individuals that might previously have been more difficult to establish (i.e. with individuals located in different geographic regions), it cannot provide the same depth of trust that can be secured through face-to-face contact.

This study also found that organisations participating in inter-organisational collaboration may carry out similar activities but use different software applications to facilitate these activities. For example, in Case 1, the research teams in the two organisations both undertake quantitative analysis of data but use different statistical analysis packages to facilitate this. The impact of this is that datasets must be imported from one software package to another, thus adding an additional step or level of complexity in sharing data.

To a large extent, the availability of ICT tools is dependent on the experience and influence of the inter-organisational team leaders. Indeed, the availability of ICT tools in Cases 2 and 3 results from decisions by the respective leaders of these Cases, both of whom had positive experiences of using such tools in inter-organisational teams. In Case 3, Rebecca indicated that she had led several cross-sector collaborative ventures 
and was keen to use shared ICT tools. Similarly, Martin (Case 2) is heavily involved in Justice-related inter-organisational collaboration. He reports that Jus1 is one of the major users shared workspaces within the sector. By contrast, those in leadership roles in Cases 1 and 4 demonstrate low levels of awareness of tools such as shared workspaces, having had no direct experience of them. This suggests that the availability of collaboration tools in inter-organisational teams is dependent on awareness and prior use of tools at a leadership level.

The limited availability of ICT tools was a surprising finding given the distributed nature of three of the research Cases (Cases 1, 2, 4). In virtual teams, geographic distance between individuals necessitates the use of ICT to provide the links and information to enable them to work together (Lipnack \& Stamps, 1997 in Pauleen, 2003). In addition, approaches to information and knowledge sharing in the New Zealand public sector have been largely dominated by the development of ICT-related initiatives that were primarily driven from the E-government strategy ${ }^{22}$. The overarching aim of the strategy is to support public sector organisations to work together to integrate services and facilitate information sharing (E-government, 2006). However, most participants report limited awareness of the E-government strategy and its related initiatives. Further, those who are aware of the strategy are unsure how it relates to them, or could support their work.

Previous research has posited that ICT may positively affect knowledge sharing. In the early research into knowledge sharing, Hendriks (1999) suggested that ICT tools may influence an individual's motivation to share. This theory is not supported in this study, where no Case participants report that ICT tools motivate them to share with others. Rather, the predominant benefit of ICT relates more strongly to Davenport's finding (1994) that ICT provides initial connections to individuals to whom they otherwise may not be connected. As the Cases illustrate, ICT tools such as email provide a communication mechanism for individuals engaged in inter-organisational sharing, but

22 At the time of data collection the E-Government Strategy had been in operation for approximately 6.5 years. 
participants do not communicate with others simply because the channel is available; rather because they have a specific need for interaction with an individual. As Cases 1 and 4 indicate, even with the availability of email, communication is often limited to individuals from the same parent organisation.

\subsubsection{ICT use}

The research found no direct association between the availability of ICT tools and the use of ICT tools. That is to say that, even when an ICT tool such as a shared workspace is available, this does not necessarily mean that it will be used (see Cases 2 and 3). Rather, the use of ICT tools is influenced by several factors including the nature of the knowledge that is to be shared, the physical proximity of the recipient, perceived ease of use of the tool, and the technical ability of the individual. Of these, the perceived ease of use of the tool and an individual's technical ability are the most influential factors.

\section{Perceived ease of use of the ICT tool}

In this research, the uptake and use of ICT tools, such as shared workspaces, is shown to be greatly impacted by participants' perceptions of difficulties associated with them. These perceptions result both from participants' actual experiences of shared workspaces and from perceptions that have developed as a result of the reported experiences of others.

In Case 3, participants report that they do not use the workspace because it is considered to be difficult to access and navigate. In this Case, several participants base their view on previous experience with workspaces. Further, because information is also shared via alternative methods such as email, the workspace is not seen as adding value to the collaboration. In Case 2, the extent to which the workspace is used varies between individuals, and functionality is largely limited to the posting of documents, despite the availability of a shared calendar and discussion forum. Several Case 2 participants report specific frustrations with the usability of the workspace particularly in regard to logging on, and the need to duplicate work because of specific technical limitations of it. These findings are consistent with the Technology Acceptance Model (TAM) first proposed by Davis et al. (1989), which identified that individuals' perceptions of both 
the ease of use of technology, and the perceived usefulness of the technology, influenced the actual use of technology.

\section{Individual technical ability}

The use of ICT tools is also impacted by an individual's level of ICT awareness and technical ability.

At a leadership level, Rebecca (Case 3) and Matthew (Case 2) demonstrate a breadth and depth of knowledge regarding the e-Government strategy and its related initiatives. They also indicate that they support the use of tools such as shared workspaces, and actively work to make them available to team members. However, as the lack of use of the shared workspaces in these Cases shows, leadership endorsement alone cannot overcome perceived difficulties in using the tools.

Participants who hold data- and information-related roles, such as those in Case 2, are more confident about discussing ICT and the way in which it could be used to support inter-organisational sharing. Some participants also indicate that they have a belief in the use of technology but that there are pitfalls that need to be overcome or avoided before technology use can become more widespread. However, the majority of participants demonstrate limited awareness of the e-government strategy and related technology tools such as shared workspaces, or other collaboration tools. In most instances, several participants indicate that they had heard of e-government but were unfamiliar with what that actually means and how it relates to them. This is particularly the case with participants in policy- and research-related roles. Further, as the individual Case studies illustrate, participants indicate that, in previous instances, tools have been available but a lack of training about how to use them has limited participant's confidence and willingness to use them. This is endorsed by Rebecca who believes that the lack of use of the Shared Workspace in Case 3 was due to issues related to difficulty in making the workspace available, and the absence of any technical training for users. 


\subsection{Knowledge sharing and social capital}

A key motivation for conducting this study was the exploration of SC and its relationship to knowledge sharing. This section analyses the findings relating to SC across the four Cases to identify over-arching findings relating to the phenomenon.

As detailed in Chapter 2, SC is commonly discussing using the relational, cognitive, and structural trichotomy (Chow \& Chan, 2008). This categorisation was noted in the conceptual framework guiding this study, and was used as a structural guide for presentation of the results within the individual Cases. Accordingly, the discussion of $\mathrm{SC}$ in this Chapter is separated into sub-sections that address each dimension.

\subsubsection{Relational social capital}

Relational SC is commonly studied as a possible determinant for why people share knowledge, and focuses on aspects of trust, identity, and the role that norms, obligations, and expectations play within a group (see Chua, 2002; Huysman and de Wit, 2004; Inkpen \& Tsang, 2005).

\subsubsection{Trust}

In this research, trust was explored through both the qualitative and quantitative phases of the study. Qualitative data regarding all participants' perceptions of trust types, trust building, and levels of trust in each of the Cases was gathered through the survey. The interview process explored these issues in more depth with a number of individual participants. This provided the researcher with greater clarification and understanding of this issue and its role in this research. The multi-method approach also reveals instances where the findings derived from one research method (the survey) contradict the evidence derived through another method (the interview process). This was particularly evident in the analysis of trust and is reflective of the types of issues that are evidenced within the Cases. For example, participants report that trust is not automatically present at the inception of the inter-organisational team, and needs to be established during the duration of the collaboration. Similarly, the interview process enabled a level of trust to be developed between the participant and the researcher and so themes, such as trust, were able to be explored in more detail. However, in some 
instances, a participant's only engagement with the researcher was through the introductory letter and questionnaire; as such there was no opportunity for trust to be established between the participant and the researcher.

Participants' assessment of trust levels within their respective inter-organisational teams identify that most participants in Cases 1 and 3 consider trust levels to be above average. However, sixty percent of Case 2 participants and forty-five percent of Case 4 participants returned a neutral response to this question, suggesting potential issues with trust in these Cases. These issues were substantiated during the subsequent interviews with participants in these Cases.

Case participants confirm that, as Luna-Reyes et al (2004) assert, trust is an important aspect of inter-organisational collaboration and contributes to the level of information and knowledge sharing that occurs within such endeavours. Trust is considered important by all participants, and is identified as the second most important motivator of knowledge sharing behaviour (see Table 57). Participants also observe that trust-related issues are one of the most problematic areas for inter-organisational collaboration (see Appendix 18). In particular, survey respondents note factors such as, "lack of trust between individuals"; difficulties associated with, "establishing and building trust amongst team members"; and, "a fear of losing face, or losing control of information" (see Appendix 18). The interview process augments these perceptions with more detailed narrative that identifies risk relating to the unauthorised release or sharing of information with parties including the media, the Minister, or within a recipient organisation; and identifies that the focus on organisational accountability inhibits sharing in the inter-organisational context.

The issue of organisational accountabilities has been raised in prior studies (see Taylor \& Wright, 2004) and the need for appropriate accountability frameworks has been highlighted in two internal investigations of collaboration in the New Zealand public sector, namely the Pathfinder Project (2001) and the Factors for Successful Coordination framework (2008). This latter framework was developed as an outcome of work relating to the Government's development towards the goal of "Co-ordinated State Services" and clearly differentiates between instances of organisational co-ordination and organisational collaboration. Importantly, the framework states that in cases of co- 
ordination, projects should encounter minimal accountability issues (SSC, 2008). This is chiefly due to the fact that co-ordination does not require changes to organisational authority or accountability; rather it affords organisations the opportunity to share information and expertise to improve the development of policies, and aspects of programme and service design and delivery. All of the Cases detailed in this study are representative of examples of co-ordination and as such, there should be limited evidence of knowledge sharing being inhibited or restricted due to issues of organisational accountability. However, as this study shows, this is not the case. Examples cited in Cases 1, 2, and 4 clearly show that inter-organisational knowledge sharing can be negatively affected by conflict relating to an organisation's accountability within the sector, and to an individual's accountability to their parent organisation. Similarly, in their study of organisational readiness for knowledge sharing in the sector, Taylor and Wright (2004) found the individual performance of an organisation to be at variance with the requirement inter-organisational collaboration. At an individual level, despite participation in the inter-organisational endeavour, an individual's primary line of accountability remains with their parent organisation. This can result in instances where information may be deliberately withheld due to the risk associated with sharing it with other Case members as illustrated in Case 1 (p. 118) and Case 2 (p. 160). This, in turn, can be perceived by other team members as an unwillingness on the part of the individual to share with others. However, as the research shows, this is more commonly due to organisational level issues such as a lack of management support or formal sign-off to share knowledge, or conflict between the purpose or goals of the inter-organisational team and those of individual organisations, as best illustrated in Cases 1, 2, and 4 .

The need to build trust between individuals is noted by participants across each of the Cases $^{23}$. This supports the assertion that the establishment of trust is a precursor to the growth of SC and the development of relationships (Cohen \& Prusak, 2001; Cote and Healey, 2001).

\footnotetext{
${ }^{23}$ However, only Case 3 participants reported evidence of actual initiatives or events to help establish trust between team members.
} 
Twenty-nine percent of participants note that trust can be established by getting to know an individual. However, this research found that, once assembled, there was little or no focus on enabling or supporting individuals to become familiar with other team members, on either a formal or informal basis. This is despite the fact that, apart from instances where individuals came from the same teams within their parent organisations, only two percent of participants indicated that they knew of another team member prior to joining the collaboration. Wu et al. (2007) describe opportunities for getting to know other team members as social interaction activities designed by team leaders to promote knowledge sharing. In this study there is little evidence of this kind of designed interaction other than in Case Three where there was an example of a purposively designed opportunity for SC to be developed. In general, Case participants are unsupportive of formal team-building activities, particularly those conducted outside working hours or via post-work social functions. One reason for this lack of support is attributed to time constraints, already an issue for many Case participants, particularly those in Cases 1, 2, and 4 where Case-related activities are undertaken as part of individuals' day-to-day roles. Some participants note instances of informal interaction, for example, drinks after work; however these occasions are not common and often involve a limited number of individuals, rather than across an entire team. This view presents a clear paradox. On the one hand participants believe that trust is an issue and that there is a greater need to build trust; on the other hand, the majority do not support focused efforts to facilitate trust-building. Although, Case 3 participants are somewhat supportive of the specific efforts made by Rebecca (team leader) to provide opportunities for individuals to get to know each other at the outset of the collaboration, participants consider that the co-location of the team was a considerable aide in enabling them to get to know each other within the confines of the collaboration and without the need for formal activities.

As articulated through both the survey and through individual interviews, trust building is also facilitated through the strong sense of commitment to a collaboration. Much of this commitment is derived from individuals' belief in the importance and value of the work in which they are engaged. As noted in the individual Cases, it is often this belief in the intrinsic value of their work that drives individuals to share and, in some instance, can help to overcome trust-related issues. It represents a level of belief in the overall 
nature of the work such that individuals are prepared to work towards these goals for the sake of the objectives themselves. Wu et al. (2009) describe this as altruistic trust, and posit that this type of trust is less reliant on trust in colleagues. This altruism is demonstrated through several participants' references to the importance or value of the subject domain in which they work (see p. 136), and the significant length of tenure within their respective fields (see Appendix 7). In several cases, participants note that they have been working in the field of education or justice in excess of a decade or more and are committed to achieving sector-related outcomes.

Evidence of trust and SC within a population can be determined by the extent to which a population is characterised by reciprocal ties (Hanneman \& Riddle, 2005). Accordingly, trust within each Case was also assessed based on the frequency of interaction and levels of reciprocity (see Table 62). Cross-analysis of these measures of participants' assessment of trust levels within their teams shows reciprocal ties to be highest in those collaborative endeavours where participants perceive trust to be above average (Cases 1 and 3). Lower reciprocity scores were recorded in Cases 2 and 4, where the qualitative evidence also identified trust-related issues.

Table 62: Reciprocity measures

\begin{tabular}{|l|r|}
\hline Case & Reciprocity \\
\hline Case 1 & $37.50 \%$ \\
\hline Case 2 & $32 \%$ \\
\hline Case 3 & $42 \%$ \\
\hline Case 4 & $19 \%$ \\
\hline
\end{tabular}

Case 2 demonstrates the second lowest level of reciprocity across any of the Case networks, despite the fact that the inter-organisational team comprises fewer members than any of the Cases. Theoretically, in small networks, such as Case 2, it should be easier for individuals to build trust and establish reciprocal ties. A lack of trust is also evident in Case 4, but is predominantly confined to the two central organisations within the Case (Health1 and Health2). Similarly to Case 2, several participants did not respond, or returned a neutral response to the survey question regarding trust levels, and the SNA recorded a reciprocity measure of only $32 \%$. The highest levels of reciprocity are demonstrated in Case 3, the only co-located team, and Case 1, which involves only two organisations. 
Findings indicate that reciprocal ties are more likely to occur between participants from the same organisation than between participants from different organisations. This suggests that trust levels are likely to be higher between participants that are employed with the same parent organisation. However, as illustrated in Case 2, trust engendered through organisational belonging can be offset when individuals are employed within different business units within an organisation. Thus, where individuals derive from the same parent organisation, trust is more likely to occur when these individuals work together within the parent organisation than if they derive from different business units. Given that organisational teams are commonly co-located, this supposes that physical proximity is a stronger antecedent of trust than organisational belonging. Further, when team members are co-located as they are in Case Three, then reciprocal relationships exist across the team network, rather than at the organisational level. Thus, physical proximity to team members is an important dynamic of trust establishment in the context of inter-organisational collaboration.

Trust is also demonstrated through clique membership. Cliques represent a subgroup of a network in which the actors are more closely and intensely tied to one another than they are to other members of the network (Hanneman \& Riddle, 2005). Table 63 reports the number of cliques present within each Case.

Table 63: Cross-case clique analysis
\begin{tabular}{|c|c|c|}
\hline Cases & $\begin{array}{c}\text { No of } \\
\text { Organisations }\end{array}$ & $\begin{array}{c}\text { No. of } \\
\text { Cliques }\end{array}$ \\
\hline Case 1 & 2 & 3 \\
\hline Case 2 & 5 & 1 \\
\hline Case 3 & 7 & 8 \\
\hline Case 4 & 8 & 22 \\
\hline
\end{tabular}

Analysis of clique membership in this research shows that in Cases 1 and 2, and, to a limited extent Case 4, the basis for membership of a clique relates to organisational belonging. For example, in Case 1, membership is dominated by Ed1 participants. Similarly, in Case 2, the single clique comprises only members of Jus1. Case 4 contains a much higher number of cliques than the other Cases, and while membership involves participants from a range of organisations, Health1 participants are most dominant. As Table 63 shows, there is also some evidence that Cases that involve greater numbers of 
organisations will reflect greater numbers of cliques, as seen in Cases 3 and 4. Thus, Cases that involve a high number of organisations may result in greater complexity due to higher numbers of sub-groups operating within the inter-organisational context.

As noted above, trust levels are highest within Case 3. This Case features several aspects that differ from the other Cases in the study. Case 3 includes a focus on team design and collaboration structure that is not evident in other cases. This focus includes purposeful decisions relating to the identification and selection of Case participants and the nature of their involvement (part-time or full-time), as well as consideration of the location of team members, as well as an awareness on team building. These factors provide a key point of differentiation between this and the other three research Cases. In Cases 1, 2, and 4 where participants are selected based solely on their availability and the role they played within their parent organisations; these participants carry out duties as part of their normal day-to-day activities rather than as a full-time engagement; nor are they co-located. In addition, there was not any formal awareness of focus on team building.

\subsubsection{Identity}

Identity represents a sense of connectedness between individuals (Widen-Wulff \& Ginman, 2004) and enables individuals to see themselves at one with another person or social group (Nahapiet \& Ghoshal, 1998). In this research, identity was explored through both SNA and interviews with participants.

In SNA, connectivity is associated with the notion of group membership; in this research SNA was used to measure the overall connectivity or cohesiveness (density) of each of the inter-organisational teams (see Table 64). The results show that Case 3 participants report the highest level of cohesion across the team. These connections are characterised by strong ties and high levels of reciprocity (see Table 62 above), and typify a group that is highly cohesive and embodies a strong sense of group identity. By comparison, other Cases demonstrate both lower density and lower reciprocity measures. The lowest density measure is recorded by Case 4 and this corresponds to the qualitative findings from the Case that observe that, in this Case, there has been little 
focus on building a team identity. Rather, the focus has been on establishing the work of the inter-organisational team within the wider health community.

Table 64: Cross-case density \& reciprocity m
\begin{tabular}{|l|l|l|}
\hline Case & Size & $\begin{array}{l}\text { Cohesion } \\
\text { (Density) }\end{array}$ \\
\hline Case 1 & 12 & $33.33 \%$ \\
\hline Case 2 & 10 & $32.22 \%$ \\
\hline Case 3 & 14 & $46.15 \%$ \\
\hline Case 4 & 20 & $16.32 \%$ \\
\hline
\end{tabular}

Kramer, Hanna, Su, \& Wei (2001), posit that an individual's level of identification with a group influences their decisions about whether to engage in trusting behaviour. Higher levels of trust are likely to result in strong ties and high levels of interaction within a team, so strengthening the overall sense of group identity. This research found that a sense of group identity is closely associated with levels of trust within each Case. Where trust levels are lower (Cases 2 and 4), participants demonstrate weaker identification with the inter-organisational team, both through the social network measures and individual comments made during the interview process. By comparison, in Case 3 which scored the highest social network measures, individuals vocalise their sense of belonging to the team.

Accountability also influences an individual's ability to identify with a group. As evidenced in the previous section, participants' capacity to share knowledge is influenced by their accountability to a parent organisation. This is most clearly articulated by participants in Cases 1 and 2 who emphasise/note/acknowledge that the decision to share information and knowledge must always consider the impact on the organisation, as well as whether the organisation has provided a mandate to share. In these instances, an individual's sense of identity is most strongly aligned with the parent organisation, and this can be construed by other individuals as unwillingness to share, or a lack of identity with the inter-organisational team.

The research also suggests that identity is influenced by an individual's role within a Case, specifically in terms of the point at which an individual becomes involved with the inter-organisational team, and the nature of that involvement. In each Case, those holding leadership positions identify strongly with the inter-organisational team, and 
indicate a sense of responsibility for the team. Common factors across Case leaders included their involvement from the inception of the Case, and expertise at a strategic level. Thus, the earlier and deeper an individual's involvement in an interorganisational team, the stronger is the likelihood that they will feel a sense of alignment and identity with the inter-organisational team. From an individual perspective, social identity theory links leadership with contributing to the development of individuals' sense of group identity (Ashforth \& Mead, 1989). To a large extent, the sense of identity across the inter-organisational teams is contingent on the Case leaders. This is most clearly evidenced in Case 3 where Rebecca's leadership of the team is a contributing factor to the strong sense of group identity that is reported by individuals.

The results of Case 4 provide a different perspective on identity. In this Case, considerable resources are allocated to support the creation of an identity for the collaboration, rather than the team. As the social network measures show, there is limited connectivity across the network, particularly between individuals from different organisations. Rather, the focus of the central co-ordination team (Alexa and Jane) is on building awareness and support for the collaboration across the wider health sector. To this end, considerable energy and resource has been expended in delivering a national road-show, making information available through a website, and engaging in one-onone discussion with health practitioners and other representatives. Thus, the aim of this team is to establish an identity for the "collaboration" rather than a sense of group identity across team members.

\subsubsection{Norms, obligations, and expectations}

Feldman (1984) posits that groups adopt norms to regulate behaviour. These norms are developed over time and informally, and result from group members learning what behaviours are required to enable effective group functionality.

The cross-case analysis of the data identified two predominant findings. The first indicates that the establishment of group norms is influenced by the level of formality within the Case. The second finding relates to participants' expectations that knowledge sharing will be reciprocal. 
The level of formality refers to the presence of formal processes and procedures that determine how work will be done. These processes provide standardisation and consistency in the way in which work should be carried out in the collaboration and, in some instances, can be viewed as the rules of engagement for Case participants.

The highest level of formality is evident in Cases 2 and 4. Both Cases are guided by formal terms of reference that determine the overall purpose of the collaboration, as well as other information such as the main roles and responsibilities of the interorganisational team. Case participants also report that the main activities of the team are guided by processes that have been formally documented and shared with team members. Examples of documented processes include the change control system in Case 2, and the analysis of data in Case 4. These Cases also share similarities in regard to the way in which the formal processes were developed. In both Cases, an initial lack of formality led to difficulties in respect to some aspects of the Cases. For example, in Case 2, an absence of formality resulted in a lack of documentation regarding decisionmaking, and a haphazard approach to sharing. In Case 4, early issues related to decision making and control resulted in the development of a mandated terms of reference and a range of formal processes. However, participants report that the formal approach to the Case had also caused some issues and, in Case 2, had effectively "killed the real collaboration”.

By comparison, Cases 1 and 3 have adopted a less formal approach, and are more reliant on individuals to make choices and decisions about how work is undertaken. This is particularly evident in Case 3, where the team leader (Rebecca) reports that she relies on the expertise and experience of individuals to self-manage and ensure that work is completed in an effective and efficient manner (see page 215). As a result, the group has established its own norms in regard to the way that work is carried out. This is supported by the co-location of team members, as well as the full-time nature of their engagement within the collaboration.

Eighty-four percent of Case participants report believing that knowledge sharing would be reciprocal. However, as the SNA shows, the actual levels of reciprocity within each Case indicate that this expectation is largely unfulfilled. 


\subsubsection{Cognitive social capital}

\subsubsection{Shared language}

The concept of shared language was explored through the interviews and the survey. The importance of shared language has been identified as a key consideration for knowledge sharing (Chua, 2002; Huysman and de Witt, 2002; Ipe, 2003; Nahapiet \& Ghoshal, 1998). However, participants in this study do not perceive the lack of a shared language as one of the most significant knowledge sharing barriers. The results reveal that both Cases 2 and 4 are focused on the importance of consistent terminology across their respective sectors, while shared language was found to be a difficult issue for Case 1 participants. There are no significant findings in relation to Case 3.

In Cases 2 and 4, a focus on shared language enables Case participants to evaluate concepts and share information based on the use of agreed terminology and definitions. This is most effectively demonstrated by Case 2 whose sole purpose is to ensure that terminology is consistent across the Justice Sector and related organisations. Though it might be expected that the increased sophistication of technology has reduced issues relating to language, Case 2 shows that, in the Justice sector at least, the need for shared terminology has increased due to the decentralisation of information systems across the sector. Similarly, in Case 4, the development of specific performance indicators has provided the sector with a set of common codes and definitions by which organisational performance can be measured.

Difficulties associated with a lack of shared language are most prevalent in Case 1, where there is no common language or use of terminology between the two organisations. The issue is made more complex by the fact that the same terminology is used by each organisation but is measured and/or defined differently. This was found to both limit information and knowledge sharing, and to increase the time participants engage in the Case to ensure that information is not misinterpreted. While the issue and associated problems are clear to Case participants, they are unaware of any action by either organisation to develop any shared terminology between the two and consider that this would be a considerable undertaking for the two organisations. 


\subsubsection{Shared purpose and goals}

Participants identified that the need for shared purpose and goals is a challenge for most inter-organisational endeavours (see Appendix 18). The survey responses indicate that sixty percent of participants in Case 2 identify difficulties related to the development of shared goals, and acknowledge that they sometimes have difficulty balancing the interests of their own organisation with the interests of the inter-organisational team. While the survey responses from other Cases do not identify this as an issue, further exploration of the issue through interviews with individuals reveals that an individual's ability to commit to shared goals is sometimes hindered by accountability to their parent organisation. For example, some participants note that the presence of individual agendas conflicts with the goals of the inter-organisational team, and thus limits individuals' ability to commit to the inter-organisational team goals. These agendas may stem from individuals' sense of what is important, or may be guided by their parent organisations' wants and needs. For example, in Case 4, participants indicate that a lack of trust between the two co-ordinating organisations (Health1 and Health2) initially impaired the development of a sense of shared purpose. Similarly, in Case 1, a lack of trust between Edu1 and Edu2 also impacted participants' ability to define shared goals and outcomes for the team. Participants also report that, in general, when a shared sense of purpose is not agreed upon, this can place restrictions on their ability to share information from their organisation. Thus participation in a collaboration can place individuals in a position of conflict between the requirements of their parent organisation and the requirements of the inter-organisational team. By contrast, in Case 3, despite the relatively large number of organisations participating in the collaboration, individuals report that they are able to successfully commit to the collaboration goals without compromising their responsibility to their individual organisations. Underlying reasons for Case 3 findings stem from a combination of leadership decisions, and participants' belief in the intrinsic value of the work being undertaken. In this Case, leadership decisions regarding the form of the collaboration resulted in the purposeful selection of participants and their co-location which has enabled familiarity and trust to develop between individuals, as well as an initial focus on team building. 
These findings lend support to the distillation of shared purpose into two distinct factors (D’Amour, Goulet, Labadie, Martin-Rodriguez, \& Pineautl, 2008): shared goals represent the ability of the group to identify and work towards a single shared goal, while allegiance refers to their ability to juggle allegiance to a shared goal with allegiance to a parent organisation. This research has found that the intrinsic value of the work in which the inter-organisational team is engaged plays a significant role in driving participants' sense of commitment to the collaboration, and contributing to the common goals of the team.

The initial stage of a collaboration, when team members are first assembled, is clearly the time when agreement about shared goals should be addressed. However, as the previous section identifies, a lack of focus on team building, coupled with issues relating to time, often means that participants are given little opportunity to spend time focusing on the purpose and outcomes of the collaboration. Consideration of the research Cases indicates that a shared purpose appears to have been best achieved in Case 3.

\subsubsection{Shared culture}

A shared culture has been associated with a positive orientation to knowledge sharing (Van den Hooff \& Huysman, 2009). This research explored how issues of culture manifest within each of the Cases and how this affects knowledge sharing.

This study found that there are a range of different organisational cultures evident within the sector. Further, individuals' ability and propensity to share knowledge in inter-organisational teams is influenced by both the culture of their parent organisations and the over-arching culture of the sector. Overcoming cultural issues can be achieved through the nature or form of the collaboration, such as the deliberate co-location of participants and minimising the amount of formal processes and procedures.

Forty-five percent of participants in this study believe that the culture within the interorganisational team differs from the culture within their own organisation. This finding is particularly significant in Cases 3 and 4 (see Appendices $12 \&$ 14). The lowest level of reported cultural difference is found in Case 1, which represents the fewest 
participating organisations (2). This suggests that the increase in cultural diversity may be related to the number of organisations participating in an inter-organisational collaboration. Where a greater range of cultures are represented within the interorganisational team, then a greater diversity of cultural differences will be evident.

The research also found evidence of cultural issues at a sectoral level, and within subsets $^{24}$ of the sector. Several participants indicate that in addition to the role that individual organisational culture plays a role in determining the extent to which knowledge is shared, there is also the issue of the culture of the public sector as a whole. A number of participants (across all Cases) note that the culture of the public sector is seen as one that is risk averse in terms of knowledge sharing, much of this risk is driven by the needs of the respective government ministers. As illustrated in Case 1, the need to limit the risk to the Minister is seen as one reason why sharing does not always occur. In Cases 2 and 3, participants observe that, while the culture of the Justice sector is largely regarded as one of the more formal subsets of the public sector, even within the sector a range of individual organisational cultures are evident.

The survey responses indicate that despite the acknowledgement of organisational cultural differences, forty-three percent of participants indicate a high level of optimism in relation to the ability of inter-organisational teams to overcome these differences (see Appendix 17). In this respect, participants do not believe that the presence of multiple cultures should inhibit the inter-organisational team from developing a shared culture within the team. However, as with the exploration of other issues in this study, the interview process reveals a deeper level of concern than evidenced in the survey responses.

Data from interviews with participants show that the impacts of cultural diversity differ in each case. In Case 1, cultural differences are a significant inhibitor of knowledge sharing between the two organisations, and negatively impact knowledge sharing within Edu1. Therefore, in this Case, cultural issues affect both inter and intra-organisational sharing. In Case 2, cultural differences are epitomised through the formal approach to

\footnotetext{
${ }^{24}$ Sub-set relates to a specific area of the public sector, such as Justice, Education, Health etc.
} 
the collaboration, and are reflective of the type of process-driven approach that hinders knowledge sharing (Holsapple, 2004). Participants (from organisations other than Jus 1) report that the collaboration reflects a formal and process-oriented approach that is more reflective of the culture of Jus1 than of their own organisation. The level of formality is also an issue in Case 4, where the two central organisations reflect very different cultural approaches, particularly in relation to communication and sharing. Health1 is considered to be more formalised in regard to official processes and documentation than Health2 which tends towards a more informal approach characterised by a high level of communication and interaction. While Case 3 involves the second largest number of organisations, the presence of cultural variety does not impact knowledge sharing interactions within the team. This indicates that while the number of participating organisations results in a greater range of cultural diversity, it is not diversity per se that leads to difficulties in sharing knowledge.

It is clear from these findings that the development of a shared culture has been most successfully achieved in Case 3. As illustrated earlier in this Chapter, the specific form of Case 3 (co-location and full-time engagement of participants, level of formality, collaboration duration) has been found to assist trust building and the development of a sense of group identity. Similarly, the form of the collaboration is now seen to contribute to an inter-organisational team's ability to develop a shared culture.

\subsubsection{Structural social capital}

In this research, structural SC was explored using SNA, and focused on the analysis of the network configuration and ties between actors (participants). The purpose of incorporating SNA was to compare the formal inter-organisational team structure with the way in which information and knowledge was actually shared within the network. To some extent, the results of the structural SC have already been discussed in previous sections of this chapter. For example, tie strength and reciprocity was discussed in Sections 8.2 and 8.4. The primary focus of this section is to articulate the findings that have not been previously discussed. 


\section{Network level analysis}

The overall cohesiveness and level of information flow in each Case was determined through the density measure (see previous Table 64). Prusak (1998, cited in Anklam, 2003) states that in order to improve knowledge flows, it is first necessary to understand those pathways, thus the density results identify the information flows across each interorganisational team.

Case 3 reports the highest density measure indicating that team members in this collaboration enjoy a high level of connectedness where information travels quickly through the network. By comparison, only one-third of knowledge sharing ties are in place in Cases 1 and 2, and only one-fifth in Case 4. Therefore, information flow is best facilitated in Case 3, while Case 4 participants are likely to experience significant difficulties related to the disjointed nature of the network. These density measures are also reflected in the survey responses which report that participants consider some individuals in their collaboration share knowledge more easily than others.

Analysis of the density measures in respect to the differences in the nature or form of the collaborations indicates that cohesiveness is strongest in Case 3, which is differentiated from the other Cases by several factors including the purposeful selection of participants, the co-located nature of the collaboration, participants' full-time engagement in the collaboration, as well as a low level of formality within the Case.

As discussed in Section 8.4.1.2. density measures are also indicative of a group's collective identity. A higher density measure is indicative of a team where individuals display a sense of belonging with the team. In this study, Case 3 participants demonstrate a strong sense of group identity as seen through both the density measure and the comments of participants (see Chapter 7).

Network distance measures indicate how long it will take to access information if an actor is not directly connected to another (Hanneman \& Riddle, 2005). A comparison of distance measures is shown in Table 65. 
Table 65: Network distance measures

\begin{tabular}{|l|c|c|}
\hline Case & Network Size & Distance Measure \\
\hline Case 1 & 12 & 1.53 \\
\hline Case 2 & 10 & 1.692 \\
\hline Case 3 & 14 & 1.610 \\
\hline Case 4 & 20 & 2.520 \\
\hline
\end{tabular}

The distance measures indicate that information is most quickly accessed by participants in Cases 1 and 3, while Case 4 participants are most likely to experience delays. These findings correlate with the density measures shown above. As Hanneman and Riddle (2005) observe, less dense networks generally report higher distance scores: Case 4 participants are less well connected to team members than their counterparts in other Cases, and also have to traverse a greater distance to find the information they require.

Network centralisation reflects the global centrality of a network and reflects the degree to which a network is reliant on a single or small number of actors (Hawe \& Ghali, 2007). In this research, network centralisation has been determined using Freeman degree centralisation scores. Network centralisation scores for each Case are shown in Table 66.

Table 66: Network centralisation measures

\begin{tabular}{|l|r|}
\hline Case & $\begin{array}{r}\text { Degree of } \\
\text { Centralisation }\end{array}$ \\
\hline Case 1 & $56 \%$ \\
\hline Case 2 & $50 \%$ \\
\hline Case 3 & $41 \%$ \\
\hline Case 4 & $63 \%$ \\
\hline
\end{tabular}

Cases 1, 2, and 4 report a high degree of centralisation. This is most evident in Case 4 in which the centralisation measure of $63 \%$ confirms a high reliance on a small subset of actors. Where network centrality is high, the departure of these central connectors from a network poses the risk of fragmentation in the network, and can lead to poor communication and information sharing (Cross et al., 2002). The potential for fragmentation can be identified through the use of the block and cutpoint measure. A cutpoint focuses on individual actors and identifies those whose removal would result in 
the network being split into unconnected blocks. Analysis of potential cutpoints and blocks identified that Cases 2 and 4 contained potential cutpoints (see Table 67).

Table 67: Cutpoints and blocks

\begin{tabular}{|l|r|r|}
\hline Case & Cutboint & No of Blocks \\
\hline Case 1 & -- & - \\
\hline Case 2 & Martin & 2 \\
\hline Case 3 & -- & - \\
\hline Case 4 & Paul & 2 \\
\hline
\end{tabular}

In these Cases, the removal of the cutpoint actor would result in the networks being divided into two key blocks and prohibit the flow of information across the network

\section{Individual level analysis}

The previous section cross-analysed the network level characteristics of the Cases. This section focuses on the individual level measures reported in each of the Cases. Individual measures focused on three centrality measures: (1) Degree centrality (the number of incoming and outgoing information requests), (2) Closeness centrality (the extent to which an actor is close to all other actors in the network, and (3) Betweenness centrality (the extent to which an actor is situated between significant actors).

Cross-analysis of the centrality measures confirms that the formal structure of the interorganisational teams is reflected in the informal networks depicted in the social network data. The centrality measures for individuals indicate that, in almost all instances, team leaders occupy central positions within the network such that they are able to greatly influence the flow of information and knowledge within the team. The degree centrality data shows that in, almost all instances, team leaders attain higher scores than other team members. Brass (1995) explains that in organisations with pronounced vertical differentiation it should not be surprising for the informal social network to shadow the formal hierarchy of authority. This is confirmed in this research where all the networks in this research show a high degree of centralisation and reflect the formal hierarchies and structures that are in place within the Cases.

Cross-analysis of the in-degree and out-degree measures indicate that team leaders generally receive a greater number of incoming information requests than the number of requests they make of others. The analysis of closeness and betweenness centrality 
measures also confirms that team leaders are strategically positioned within the networks and are situated in close proximity to the majority of other actors. The combination of these measures confirms that team leaders are central connectors within the networks. However, while acknowledging the pivotal role these individuals play in the networks, as Cross et al. (2002) caution, over reliance on any single individual can lead to slowing of information flows across networks.

The results also identify a layer of information intermediaries within each of the Cases. These individuals are not identified within the formal hierarchy of the teams but their positions within the network indicate that they play a more significant role than all other members, except the team leaders. This is apparent in Cases 1, 2, and 3, but is most clearly illustrated in Case 3, where Tony's position in the network indicates that he is heavily relied on by team leader, Rebecca, to gather and assimilate information for her. Analysis of the out-degree measures associated with intermediary actors supports this finding with several intermediary actors achieving higher out-degree scores than both team leaders and other actors within their respective networks.

The social network data also indicate that participants who display higher levels of SC are more likely to be centrally placed with a network. For example in Case 2, Martin and Peter are diametrically opposed in terms of how they view the collaboration. Martin is concerned with aspects of SC such as trust between team members, and indicates a high level of identity with the group. Conversely, Peter demonstrates low levels of trust and is unsupportive of the group and its work. These views are reflected in their respective roles in the knowledge sharing network in Case 2 that positions Peter as isolated from other team members, while Martin is centrally located and enjoys strong ties with a number of other actors. Case 4 shows a similar result; both Jane and Alexa are strongly committed to building relationships across the inter-organisational team, and are highly skilled communicators. The social network data reflects this commitment with both holding highly central positions.

The cross-case analysis also shows that the strength of ties between actors is influenced by two key factors: organisational membership, and the nature or form of the collaboration. In Cases 1, 2, and 4, ties are more likely to occur, and to be stronger, between members of the same organisation. Thus, information and knowledge sharing 
is more frequent between these individuals than with participants from other organisations. However, in Case 3, organisational membership is not found to influence ties. In this Case, the co-location of the inter-organisational team and their full-time engagement in the collaboration enables equal access to all team members. This provides Case 3 participants with better opportunities to build SC between team members by providing direct, face-to-face access to all individuals. Thus, individuals are able to interact directly with participants and are not prohibited by issues of geographic boundaries, or lack of familiarity.

\subsection{Summary of Chapter 8}

This chapter has analysed and presented the key findings derived from the cross-case analysis of the four Cases. The chapter presents the analysis and findings collected through different research methods and analyses this data to derive key findings.

The chapter provides an analysis of each of the aspects of the research as they relate to the key themes of SC and ICT. In some instances, contradictions were found between data gathered through different methods. Exploration of these issues confirms the value of the use of a mixed method design and has enabled the researcher to identify and further analyse these issues.

The analysis also identified several distinct factors that influenced knowledge sharing within the Cases including: (1) the nature or form of the collaboration; (2) the intrinsic value of the work; (3) leadership.

The cross-case analysis also determined that the availability of ICT tools was influenced by the technical awareness of understanding of inter-organisational leaders, and that the use of ICT within the Cases was influenced by (1) the perceived ease of use of the tool; (2) the perceived usefulness of the tool, and (3) participants' level of technical ability.

The next chapter reviews the conceptual framework presented in Chapter 2 of the study, and provides answers to the research questions posed in Chapter 1. 


\section{CONCLUSION}

The previous chapter presented and discussed the findings from the cross-case analysis of the four Cases studied in this research. It explored the findings relating to social capital (SC) and information and communication technology (ICT) and the roles that these factors play in regard to knowledge sharing in inter-organisational collaboration in the public sector.

This chapter draws the research to a close by presenting the overall conclusions of the research. The chapter reviews the purpose of the research and re-introduces the conceptual framework presented in Chapter 2. Key findings of the research are reviewed and incorporated into the framework. The chapter then discusses the contribution of the research to both the academic research literature and to practitioners, and outlines the implications of the research. It concludes by identifying limitations of the study and discussing potential areas for future research in this field.

\subsection{The nature of the research}

This research set out to deepen understanding about the dynamics of interorganisational knowledge sharing by gathering empirical evidence about how knowledge is shared in inter-organisational collaborative endeavours in the public sector. More specifically, the study explored the roles that SC and ICT play in supporting and/or influencing knowledge sharing within inter-organisational teams. The research was conducted as a multiple case study involving four inter-organisational teams based in the education, justice, and health domains of the New Zealand public sector.

Exploratory study is often conducted where little is previously known about a phenomenon and so suggests an act of learning on the part of the researcher. Ryan (2006) suggests that post-positivism, a philosophical stance that facilitates learning, is well suited to studies of this nature. Previous research into knowledge sharing identified important a priori factors, but there had been little research that explored these in the public sector context. The adoption of a post-positivist philosophical stance supported the exploratory nature of the study, allowing exploration of these a priori 
factors within the study's specific context (Creswell, 2003), and enabling the researcher to focus on both factual evidence and the context within which it occurred (Ryan, 2006). This perspective, together with the multiple-case, multi-method approach, was also useful in revealing and exploring the sometimes contradictory research findings (Richie \& Rigano, 2001 in Ryan, 2006).

The study of multiple cases following a literal replication strategy (Yin, 1993), together with multiple research methods also helped to strengthen the generalisability of the findings (Maxwell, 1992). Although this approach was more lengthy and complex, it resulted in multiple datasets that provided a strong base for cross-case analysis (Lincoln \& Guba, 1985; Miles \& Huberman, 1994). The combination of both qualitative and quantitative data enabled more comprehensive data triangulation (Yin, 1989) and provided a greater level of validity than either a single method or single case could have established.

\subsection{The conceptual framework}

The conceptual framework, originally presented in Chapter 2, was developed following an extensive review of the extant literature. It enabled the researcher to encapsulate the ideas and concepts gained from the literature review, and distil these into a coherent framework to help guide the research. The initial framework is shown again in Figure 32.

The initial framework encompassed four main themes traversed in the literature. It identified the context of the study as the New Zealand public sector, and introduced inter-organisational collaboration as the particular area of focus. Based on the extant literature, the initial framework identified SC as a key influencer of knowledge sharing. More specifically, the framework encompassed specific aspects of the relational, cognitive, and structure dimensions of SC, and posited that these aspects might contribute to knowledge sharing in the public sector inter-organisational context. The framework also identified that historically ICT has been purported to play a key role in knowledge sharing, though scholars are divided about whether ICT is an enabler or a driver of knowledge sharing behaviours (see Hendriks, 1999; Huysman \& Wulf, 2006; Smith \& McKeen, 2003). The ICT literature also noted a potential association between 
ICT and social capital, whereby ICT may positively affect knowledge sharing, and facilitate the development of social networks (Huber, 2001; Sproull \& Kiesler, 1991).

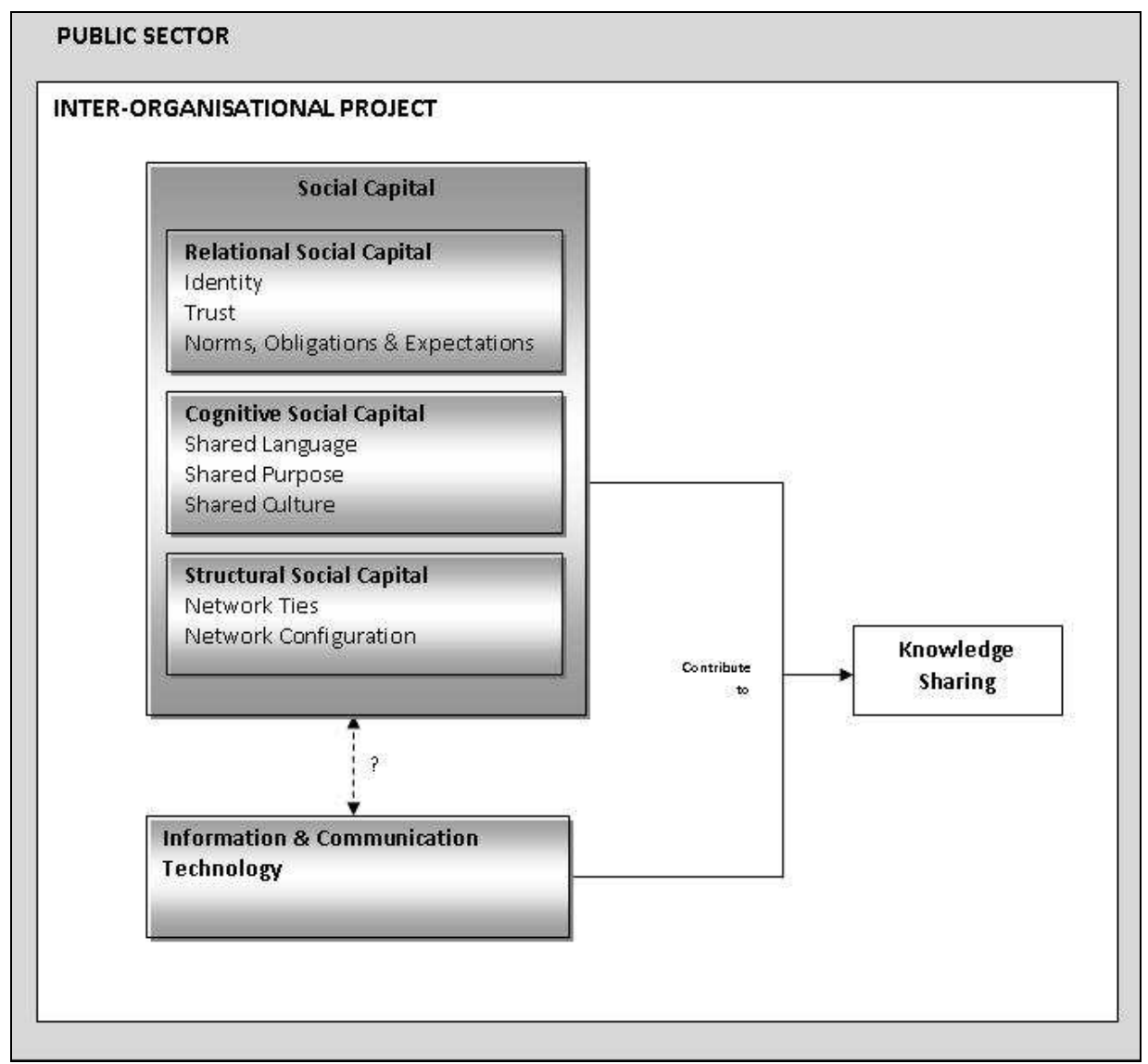

Figure 32: Initial Conceptual Framework

The following sections review each of the components of the conceptual framework based on the key findings from the research. SC, ICT, and their relationship to knowledge sharing were discussed in the previous chapter. These areas are briefly revisited in this chapter, but the predominant discussion is focused on the important identification of six key antecedents that have a bearing on the roles of social capital and ICT within the collaborations. These antecedents were identified during the crosscase analysis process where they were found to be common across several cases, and therefore of key significance to the research. They include: (1) collaboration design, (2) leadership, (3) the intrinsic nature of the work, (4) perceived ease of use of ICT, (5) perceived usefulness of ICT, and, (6) individuals' level of technical ability. These factors are incorporated in the revised conceptual framework presented later in this chapter. 


\subsubsection{The nature of inter-organisational knowledge sharing in the public sector}

This study's primary research question asked: How is knowledge shared in interorganisational teams in the New Zealand public sector? Chapter 8 provided a detailed analysis of the knowledge sharing activities available to inter-organisational teams and the ways in which these activities are perceived and used. This section focuses on the over-arching context for knowledge sharing in this study: the public sector and specifically inter-organisational collaborations as the environment for the study. The public sector was identified due to its importance to the development of national knowledge economies (Hearn \& Rooney, 2002), and the need for organisations within the sector to exploit their knowledge reserves through effective knowledge sharing (Willem \& Buelens, 2007). This need has grown as the focus on inter-organisational collaboration has increased (Walker, 2004). Yet, as identified at the outset of this research, there is a lack of empirical evidence relating to the public sector, particularly at an inter-organisational level.

This research contends that the context for inter-organisational collaboration and knowledge sharing within the NZ public sector is encompassed within three related perspectives (see Figure 33).

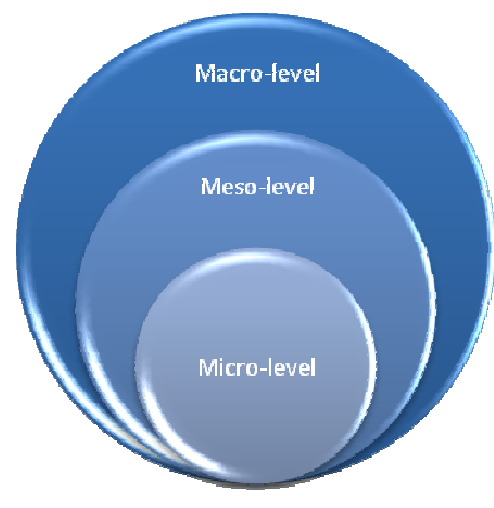

Figure 33: Context for inter-organisational knowledge sharing

Firstly, the macro-level perspective defines the government's approach toward interorganisational collaboration and knowledge sharing at a sector level; it determines the over-arching conditions and regulations that determine how collaboration between public sector organisations will take place. Theoretically, when collaboration and 
knowledge sharing takes place between two or more organisations, it should be conducted in accordance with the conditions of the macro-level perspective. Secondly, the meso-level perspective identifies the specific collaboration mechanism (within the macro-level perspective) that is developed to support the collaborative effort; for example, an inter-organisational team. Lastly, the micro-level perspective denotes the range of activities that are available to participants within the collaboration mechanism, and are used to facilitate inter-organisational knowledge sharing; for example structured meetings, and ICT tools. Together, these perspectives provide an overall context in which inter-organisational knowledge sharing can take place.

\section{Macro-level perspective}

From a macro-level perspective, governments globally have developed their own overarching approaches to collaboration, commonly termed as joined-up-government (Johnson, 2005); it is within these mechanisms that information and knowledge is shared and exchanged among different organisations and parties. Johnson (2005) summarises these collaboration mechanisms as programme-based collaboration; service-delivery integration, characterised by the collection of information and services about a shared issue or customer; and a top down whole-of-government integration. In the New Zealand context, macro-level collaboration has been formalised through the government's goal of Co-ordinated State Services, whereby organisations share information, resources, and responsibilities to assist policy and strategy development, and for the design, delivery, evaluation, or adjustment of programmes or services (SSC, 2008). As illustrated in the individual Cases, each of the inter-organisational teams is participating in work that is either encompassed within, or relates to, a wider programme of work, and/or that involves issues that are of concern to a broad range of organisations. Thus, the application of Johnson's trichotomy identifies the research Cases as both programme-based collaboration (Cases 1 and 4) and integration around shared issues (Cases 2 and 3).

\section{Meso-level perspective}

At a meso-level, collaboration is facilitated through the adoption of a specific collaboration mechanism. This research found a range of mechanisms through which 
inter-organisational collaboration is facilitated. These included a discrete project with clear start and end points (Case 3), collaborative ventures that devolve from ongoing programmes of work between organisations (Cases 2 \& 4), and a short term collaboration that occurs on an annual basis (Case 1). Though several research participants often referred to their collaboration as a project, only Case 3 fits the traditional description of a project defined by Packendorff (1995) as a unique task that has both predetermined start and end dates, one or more goals and includes a range of different activities, predetermined beginning and end dates, one or several performance goals, and a number of different activities Indeed, inter-organisational projects have become both a conceptual and an organisational form in the public sector (Lofstrom, 2009), and, by definition, involve the participation of two or more organisations (Easterby-Smith et al., 2008). However, as the number of organisations involved in the collaboration increases, so too does the complexity of knowledge sharing, due to the multi-faceted nature of the diversity of boundaries, cultures, and processes involved in inter-organisational knowledge sharing (Easterby-Smith et al., 2008). In this research, Case 1 illustrates a less complex example of sharing. This is due mainly to the low number of organisations involved, and the nature of the task which involves the transfer of data between the two organisations. In contrast, Case 3 comprises a situation which requires more in-depth sharing of tacit knowledge and draws on the embedded experience and expertise of participants. Thus, collaboration occurs on a continuum, ranging from simple co-ordination that tends to be primarily information-focused, through to more complex collaboration that requires in-depth sharing of tacit knowledge.

\section{Micro-level perspective}

At a micro level, collaboration is supported through a range of knowledge sharing activities that are available to the inter-organisational team. Prior studies have shown that these commonly consist of a variety of formal and informal activities (Ipe, 2003) that are available across a range of communication channels. However, this research shows that, in the public sector, the availability of knowledge sharing activities is largely confined to formal activities in the form of structured meetings or through formal documentation such as official papers and reports. There are fewer instances of 
informal or unstructured activities, yet this type of social interaction has been found to be more conducive to knowledge sharing than formal, structured activities (Tsai, 2002; Wu et al., 2007).

The relationship between the macro-, meso- and micro-level perspectives provides the overall context within which inter-organisational knowledge sharing takes place. While it is unlikely that participants in inter-organisational teams are involved in decisions relating to collaboration at the macro-level, it could be expected that there is merit in involving them at both the meso- and micro-level perspectives. For example, involving individuals in determining the development of the specific collaboration design - that is, the inter-organisational team - provides a sense of empowerment and involvement that, as shown in the case of Rebecca in Case 3, helps to facilitate a sense of identity with the collaboration, and with its purpose and goals. Case participants were also very clear about their preferences for knowledge sharing; identifying informal activities as most conductive to knowledge sharing. Thus, involving participants at the micro-level, would likely result in the design and incorporation of activities that are conducive to facilitating optimum levels of knowledge sharing. However, as this research shows, none of the inter-organisational team members, other than the leaders of Cases 2 and 3, had any involvement in meso- or micro-level decision-making.

As detailed in Chapter 8 this study substantiates and extends aspects of earlier research that raised concerns about the public sector's readiness for knowledge sharing, and the ability of public sector managers to face the associated challenges (Taylor \& Wright, 2004). This research confirmed Taylor and Wright's assertion that the media and general public scrutiny are considered to be key risk factors in regard to knowledge sharing. Perceived risk relating to the control of information and the release or sharing of that information to other parties was shared across three of the Cases (see Cases 1, 2, and 4). The research also extends this finding to encompass risk and concern associated with the circulation of information within organisations as well as the release of information to respective government Ministers. Case 1 participants showed particular concern in this regard and recounted instances where information that had been shared between the two organisations had been circulated to staff, or released or 
communicated without the mandate of the organisation who initially supplied the information (see p. 138).

Taylor and Wright (2004) also argue that the nature of accountability within the sector, particularly the focus on individual organisational performance, presents challenges to inter-organisational knowledge sharing. In New Zealand, the 'Co-ordinated Services Goal' (SSC, 2006) clearly states that, in instances of inter-organisational collaboration, mandated responsibilities and accountabilities remain with individual organisations. In this research, that mandate limited individuals' ability to share, as illustrated in Cases 1, 2, and 4. Thus, for individuals engaged in inter-organisational collaboration, the decision to share, or not, is often dictated at an organisational level. This results in individuals being perceived as unwilling to share, when in fact this may be out of their control. Further, the responsibility to the parent organisation can inhibit the interorganisational team from developing a sense of shared purpose. This conflict and the need to reconcile accountability at an organisational level with the ability to develop a sense of collective purpose have been recognised (SSC, 2008). However, as this research shows, this appears to remain a largely notional goal. Therefore, as Taylor and Wright posit, the onus and accountability of individuals at an organisational level strongly discourages robust collaboration and directly impacts individuals' propensity for participation and sharing. It also emphasises the paradoxical nature of the need for inter-organisational collaboration while focusing on individual organisational outcomes and performance (Taylor \& Wright, 2004).

\subsubsection{Knowledge sharing and the role of social capital (SC)}

This section discusses the research findings in relation to the second research question: What is the role of SC in inter-organisational knowledge sharing in the public sector? The research found that SC is a key factor influencing knowledge sharing among interorganisational team members. Prior knowledge sharing studies, conducted in the private sector, have identified SC as positively benefiting knowledge sharing (see Chow \& Chan, 2008; Hansen, 1999; Lang, 2004; Mu et al., 2008; Nahapiet \& Ghoshal, 1998; $\mathrm{Wu}$, et al., 2009). This study contends that SC has similar potential within the public sector; research findings provided evidence of both the importance of SC as well as empirical data about the specific social capital factors (e.g. trust, shared sense of 
purpose etc.) and their interplay. Despite this fact, the research also identified that SC is rarely consciously considered at the outset of a collaborative effort. Consequently, the benefits gained in the private sector are yet to be realised in the public sector context.

Aspects of relational SC (for example, trust and identity) were generally considered to be the most important issue for individuals engaged in inter-organisational collaboration. As van Wijk, Jansen, \& Lyles (2008) report, relational SC is possibly the most important network-level driver of organisational knowledge transfer both within and across organisations. While the relationship between trust and knowledge sharing has been explored in several studies (see Lee, 2004; Molm et al., 2000; Wu et al., 2009), studies at an inter-organisational level have only recently gained prominence (see Luna-Reyes et al., 2008; Mu et al., 2008; Pardo et al., 2006). This research confirmed that trust is a key aspect of inter-organisational knowledge sharing, and is built through demonstrations of individual capability and competence as discussed by Sako (1992, cited in Newell et al., 2003). In this way, trust is determined to be most effectively built over time as individuals become more familiar with team members' expertise and experience, and are able to see more clearly the value that those individuals bring to collaboration. These interactions provide confidence that the team member is able to fulfil their role within the inter-organisational team to the required standard. These interactions are facilitated through the range of knowledge sharing activities that are available to the team (see micro-level approach, Section 9.2.1 above). As this research shows, formal activities such as structured social occasions conducted outside of working hours, are not supported by participants. Rather, trust building must be conducted within the specific temporal and practical boundaries of collaboration that, as Lofstrom (2009) notes people do not have sufficient time for developing new routines. Therefore, organisations should focus on the development of relationships within the workplace rather than through off-site specific team building exercises (Cross et al., 2002). However, the research Cases indicate that, In the context of public sector inter-organisational collaboration, the lack of time, space, and opportunity for individuals to develop relational SC negatively impacts the propensity to share knowledge. 
The relational SC aspects of trust and identity are closely linked. This study found that inter-organisational teams which reported high trust levels were more likely to feel a sense of group identity - best illustrated in Case 3. Lofstrom (2009) contends that in order to develop a sense of identity, inter-organisational projects require a task, and a formalised project name. Furthermore, teams require resourcing, funding, commitment, and shared values, and should be located somewhere that distinguishes the interorganisational team from the parent organisation/s. Some of these factors played a role in the success of Case 3, particularly the co-location of team members away from the confines of any single organisation, and the fact that the collaboration was identified by a specific name ${ }^{25}$. However, this research argues that, unlike the task and collaboration name, development of Lofstrom's latter characteristics such as commitment and shared values cannot simply be assigned but can only be developed as the inter-organisational team begins to work together. Thus, it is dependent on the development of social capital between team members.

Cognitive SC provides a shared context within which knowledge sharing can take place. Aspects such as shared culture, purpose, and language can provide a mutual cognitive frame of reference and common knowledge among the team members (Kang, Morris, \& Snell, 2007). While these aspects were deemed important within the interorganisational teams in this study, these issues did not appear as important to individuals as the relational issues discussed above. To some extent, this may be related to individuals' scope of influence. While they are able to exert a degree of influence in regard to aspects of relational SC, such as trust, they have less direct influence on cognitive SC issues such as the development of a shared language.

Although SC related issues (for example, trust, shared culture, and shared purpose) have been previously identified in the New Zealand context (see the Review of the Centre, and 'Factors for Successful Co-ordination', Chapter 2, p. 64), this research found little evidence that consideration of these issues has permeated down to an operational level.

\footnotetext{
${ }^{25}$ The project name has been omitted in the research to protect the identity of the project and its participants.
} 
So, despite a number of individual research participants acknowledging that interorganisational knowledge sharing requires a greater understanding and emphasis on SC related issues, the majority of the inter-organisational teams had been constructed with little practical regard to how SC issues would affect the team.

The study identified three key factors that can influence the SC development within inter-organisational teams. The first factor relates to the specific consideration of the collaboration design and shows that SC can be positively influenced by decisions made about the design of an inter-organisational team prior to its inception. The second factor relates to aspects of leadership within the inter-organisational team, and shows that leadership decisions and influence can help to support the development of social capital between team members. Finally, the study notes the powerful influence of the intrinsic nature of the work in which public sector employees are engaged, and the role that this plays in motivating them to share knowledge in inter-organisational collaboration. These factors are discussed later in this chapter.

\subsubsection{Knowledge sharing and the role of ICT}

This section discusses the research findings in relation to the third research question: What is the role of ICT in inter-organisational knowledge sharing in the public sector? At the outset of this research, the literature review noted scholars' interest in ICT as a knowledge sharing tool. In addition, ICT was identified as a key component of the public sector's approach to inter-organisational collaboration, and tools such as shared workspaces were envisaged as examples of collaborative technology that would support sharing behaviours and assist collaboration and networking (SSC, 2007).

In contrast to other studies (for example, see Kim \& Lee, 2006), this research found that ICT tools did not play an important role in knowledge sharing. The limited range of ICT tools available to the inter-organisational teams, and the lack of active support for their use, were surprising findings, particularly given the emphasis on ICT as a tool for supporting knowledge sharing (SSC, 2008). The findings do however confirm Dawes \& Pardo's (2002) assertion that, despite rapid advances in ICT capability, the integration of information resources across public sector organisations has proved extremely difficult. Further, these difficulties increase proportionally with the numbers 
and types of information resources to be shared (Pardo \& Tayi, 2007). This is illustrated in Case 2, where the shared data dictionary was developed to counter difficulties arising from the move away from a single information system to individual organisational systems.

Exploration of ICT in this study revealed inconsistencies between the presumed availability of ICT tools as suggested in the literature review, and the actual availability of ICT within each of the Cases. The findings indicate that the availability of ICT was, to a large extent, dependent on the technical awareness and capability of team leaders. As a result, ICT tools for knowledge sharing were available in only two Cases (2 and 3), where the respective team leaders were both experienced users of shared workspaces and had used them in previous inter-organisational collaborations. Consequently, both leaders made conscious decisions to utilise ICT tools for knowledge sharing within the Cases. Thus, ICT knowledge and capability at a leadership level is identified as a key influencer in determining the extent to which ICT tools will be available to an interorganisational team.

The research also revealed that the use of ICT tools is largely confined to their use as a communication channel, or as a medium to store information. As a communication channel, ICT was most commonly used as a means to share documents or to provide a written record of a situation or course of action. It was not regarded, or used, as a tool to share knowledge; rather knowledge was considered to be better facilitated through face-to-face communication. Daft \& Lengel (1986) suggest that the choice of a communication channel results from the combination of the channel characteristics and the content of the intended message. This was the case for some participants who indicated that the way they communicated was dependent on what they needed to achieve. This fell into two main categories. Firstly, email was perceived as useful for situations where face-to-face communication was not available, but in which the content of the message was important, or where there were high levels of risk involved. Secondly, email provided participants with the ability to communicate information, actions or decisions about a situation, enabling them to effectively finish a task, before moving onto another. This latter action enables the individual to complete the task and mentally leave it behind, resulting in lower levels of task fragmentation and stress. 
This supports the claim of Miranda \& Saunders (2003), who argue that the need for task closure drives an individual's choice of communication channel. However, though email was a highly used medium, particularly in the geographically distributed teams, it was regarded as a convenient, but potentially higher risk, channel due to the possibility of misinterpretation of the message.

This study found some evidence to link ICT and social capital development. However while the findings indicate that use of ICT tools, such as email, can support relationship building between individuals, there was no evidence to suggest that ICT influences social capital development. Some participants indicated that email was viewed as a mechanism through which relationships could be initially developed, as well as a tool to further develop social capital following initial face-to-face interaction. However, in both situations, participants deemed that the face-to-face communication is necessary at some point to provide a more durable platform for social capital development. This finding may be specific to collaboration type: as the findings from Case 3 suggest, where team members are co-located, they may be less predisposed to considering email as trust building mechanism. Thus an individual's attitude towards trust may be influenced by the specific characteristics of the collaboration and, in instances of predominantly virtual collaboration, team members may be more open to establishing or developing relationships that are supported by the use of ICT.

In summary, this section provides a better understanding of participants' perceptions and use of ICT as a knowledge sharing tool and its use within the context of interorganisational collaboration. The findings also identify that ICT availability is influenced by the technical awareness and capability of inter-organisational team leaders, while ICT use is influenced by individual technical ability, the perceived ease of use of the tool, and its perceived usefulness. These factors are discussed further in the following section.

\subsection{The conceptual framework revisited}

The previous sections reiterated the key findings from the study and noted the identification of six key factors that were related to the development of SC and the 
availability and use of ICT. This section discusses these important aspects, and incorporates them into the conceptual model (see Figure 34).

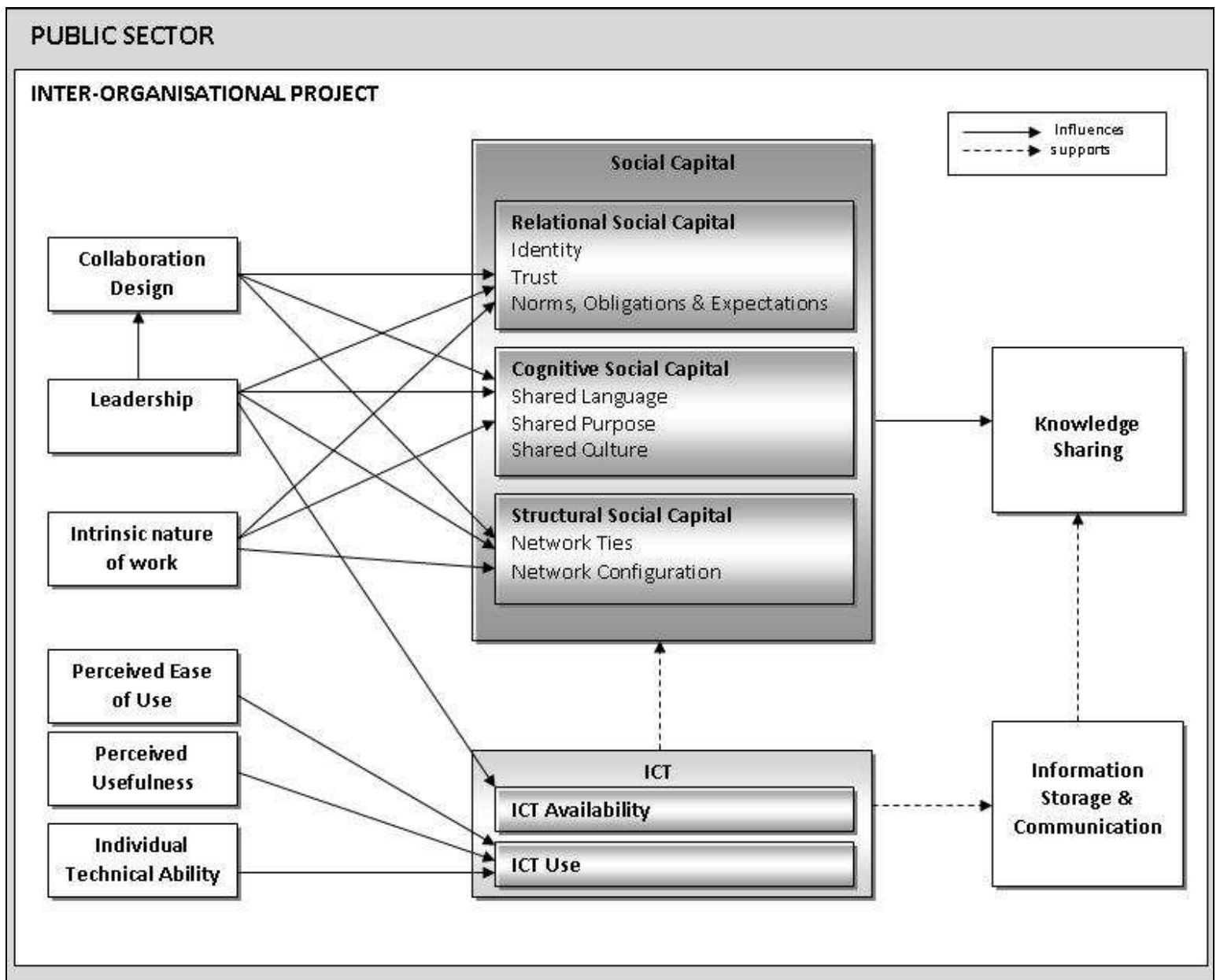

Figure 34: Final conceptual framework

The framework argues that SC influences knowledge sharing behaviours of individuals engaged in inter-organisational collaboration. The framework also indicates that while ICT supports information storage and communication between team members, it does not directly influence knowledge sharing behaviours. Rather, ICT can support knowledge sharing through the storage of information resources and through its use as a communication channel. The framework also indicates that ICT supports, but does not positively influence, SC development within the inter-organisational team.

As noted earlier in the chapter, the framework also identifies several factors that influence social capital development, and the availability and use of ICT. These factors are discussed in the following sections. 


\subsubsection{Collaboration design}

While factors such as organisational structure and culture have previously been recognised as considerations in studies of knowledge sharing (Willem \& Buelens, 2007), there has been less attention on the specific aspects of inter-organisational collaboration design.

This research defines collaboration design as actions and/or decisions considered at the outset of the collaboration that encompass factors relating to both team formation and structural elements of the collaboration including: (1) the selection of interorganisational team members, (2) the nature of team members' engagement, (3) the physical proximity of individuals, and, (4) the level of formality in place with the Case. This research found that the degree of initial focus on the collaboration design can affect subsequent development of social capital and knowledge sharing within the interorganisational team. As previously noted, Lofstrom (2009) contends that in order to develop a sense of project identity, an inter-organisational project requires a task, and a formalised name. As this research shows, in projects such as Case 1, the lack of a formal name contributes to participants' lack of identity with the collaboration, and suggests that the collaboration is not important or is less significant than those that are formally recognised. Furthermore, Lofstrom (2009) states that the collaboration requires resourcing, funding, commitment, and shared values, and should be housed in a location that distinguishes the inter-organisational team from the parent organisations. While some of these aspects were accommodated within the research Cases, only Case 3 demonstrated purposeful consideration of the majority of these factors. Further, this research argues that while task, formalised name, resourcing, and funding are factors that can be explicitly determined by team and/or organisational leaders at the outset of a collaboration, commitment and shared values require the active participation of all team members and, given the right conditions, develop over time.

In an organisational context, team formation is commonly approached from a prescriptive perspective whereby the selection of team members is the result of deliberate, strategic decisions of individuals who either self-select or assign others to a group with the purpose of satisfying individual and group objectives (Owens et al. 1998, cited in Hahn et al., 2008). As illustrated in this research, the selection of team 
members in an inter-organisational context can be more complex due to the inclusion of multiple organisations that often hold different perceptions of, and commitment to, the collaboration. As Pauleen (2004, p. 239) notes, the method of team member selection "may influence their overall willingness to be on the team, and so may require different levels of relationship building by the leader." This research identified that, in most situations, the selection of team members was determined by each participating organisation (Cases 1, 2, and 4), and while some consideration may focus on expertise, participant selection is highly contingent on individual availability. The research found no evidence to suggest that prior experience with an inter-organisational team was a consideration, nor that such experience would be formally recorded and therefore be available to assist participant selection in future collaborative endeavours. As a result, teams are often assembled in which individuals have no prior knowledge of other participants. However, research shows that teams whose members have pre-existing relationships are able to solve complex problems better than teams of strangers because they are able to pool information more efficiently (Gruenfeld, Mannix, Williams \& Neale, 1996). This is supported by the team in Case 3, which was tasked with providing alternative approaches to a complex justice issue and required a stronger focus on individuals sharing tacit knowledge (expertise and experience) than, for example, Cases 1 and 4, which dealt primarily with the exchange of information. Although some members of Case 3 had not formally worked together before, a number of participants were purposively selected by the team leader (who had previously worked with these individuals), and this contributed to the team's ability to both build trust levels and form a collective sense of team identity. Therefore, focussing on purposeful team member selection is an important aspect of collaboration design, and can be an important source of social capital growth (Hoegl, 2003).

Though not always achievable, the co-location of team members can contribute to social capital development, as evidenced in Case 3. Co-location is advantageous to interorganisational teams because it provides the opportunity for face-to-face communication and an environment that better supports trust-building, and decisionmaking (Zenun et al., 2007 cited in Loureiro \& Curran, 2007). Face-to-face communication was the preferred communication channel for the majority of Case participants despite the virtual nature of three of the Cases. Consideration of network 
density and distance measures confirm that the physical co-location of interorganisational team members provides a significant advantage in supporting knowledge sharing. Tie strength and reciprocity were weaker among the virtual teams (Cases 1, 2, and 4) than within the co-located team (Case 3). Tie strength is greater between individuals from the same parent organisation where it is likely that individuals will have shared backgrounds. Thus, as Haythornwaite (1999) identifies, individuals with similarities in attitude, background, experiences, and access to resources are more likely to enjoy strong ties. Further, this link is strongest when these individuals are in close proximity within the organisation.

A further consideration of initial collaboration design is the extent of formal process and procedure that will be introduced into the collaboration. The level of formalisation is an important factor in knowledge sharing (D'Amour et al., 2008). This research argues that the degree of formal process that is evident within the collaborative environment influences the development of social capital between individuals. For example, collaborations that exercise lower levels of formal process, such as in Case 3, enable the team to operate as a dynamic, self-organising body, where team members are able to make decisions about the ways in which they complete their respective tasks. By contrast, a strong emphasis on formal process, particularly in collaborative endeavours with greater numbers of participating organisations, acts to inhibit social capital development and results in lower levels of reciprocal sharing as evidenced in Cases 2 and 4.

This research suggests that a purposeful approach to collaboration design can have multiple benefits. As illustrated in Case 3, consideration of these aspects supported relational SC by assisting trust building which resulted in a more defined sense of group identity than evidenced in other Cases. These benefits were also evident in the structural SC of the team, which reported stronger ties, higher levels of reciprocity among individuals, and a greater fluidity in the flow of knowledge within the team. Finally, an emphasis on the design of inter-organisational collaboration helps to mitigate potential risks arising from the presence of multiple cultures within the team, and so positively affects cognitive SC development. 


\subsubsection{Leadership}

Leadership in inter-organisational collaboration occurs at both the organisational and individual levels. For example, one participating organisation is often mandated to lead the collaboration, as evidenced in Cases 2, 3, and 4 (leadership at the organisational level). In these instances, day-to-day leadership of the collaboration is designated to a single individual within the lead organisation (leadership at the individual level). In this study, individual leadership was found to be a key influencer of social capital development among team members.

Leadership research has mainly focused on understanding how qualities such as style, personality and other characteristics influence team dynamics and performance (Mehra, Smith, Dixon, \& Robertson, 2006). Earlier research most commonly focused on ongoing issues of leadership; however, as illustrated in this research, leadership decisions, for example in respect to collaboration design, can influence the way in which trust and group identity are established between team members. Erickson \& Dyer (2004) found that leadership approaches relating to initial collaboration design resulted in different collaboration outputs, despite having started with very similar inputs at initiation. This suggests that leadership intervention and involvement in the early stages of collaboration design can influence the potential outputs of the team. Engagement in the early stages of an inter-organisational collaboration, particularly during the collaboration design, also contributes to a leader's level of personal investment in the collaboration. As evidenced in the cases of both Rebecca (Case 3) and Martin (Case 2), their early involvement in the collaboration design helped to strengthen their own belief in, and commitment to their respective collaborative endeavours.

In Cases 1, 2, and 4, leadership was undertaken largely as a virtual task. Pauleen (2004) contends that virtual team leaders must manifest a different level of skill to traditional co-located team leaders. He notes that in addition to the common practice of task management, and personality conflicts, the virtual team leader must create common purpose among teams that are geographically distributed, and often comprise multiple organisations and respective cultures. This research recognises the added complexity that is associated with virtual leadership, and suggests that, even when teams are co- 
located (as in Case 3), difficulties associated with multiple organisations and cultures exist, but can be readily recognised and mediated by a co-located leader.

Leadership was also found to be an influential factor in ICT availability. This study found that where inter-organisational team leaders had greater technical capability and awareness, this resulted in a greater range of ICT tools being available to the interorganisational team. Pauleen (2003) notes that, in the virtual context, leaders who support the adoption and use of ICT play important roles as technology-use mediators in the wider organisation. This presupposes that the endorsement of technology by interorganisational team leaders should influence its adoption and use by team members. However, this research finds that individuals' perceptions of both the ease of use and usefulness of the ICT tools outweigh leadership endorsement. For example, in Case 3, team members had access to both a shared workspace and a shared drive. While participants reported support for the shared drive, none supported or used the shared workspace, despite Rebecca's strong support.

\subsubsection{Intrinsic nature of the work}

While studies have shown that trust is a key antecedent in the motivation to share knowledge (Nahapiet \& Ghoshal, 1998), this study found the predominant motivator to be related to the "intrinsic nature" of the work undertaken by the inter-organisational teams - which in this study goes beyond the boundaries of the inter-organisational collaboration and reflects the greater context or body of work in which an individual is engaged.

This finding is consistent with studies of employee motivation in the public sector, which found that employees' perceptions about the importance of the activity, coupled with their desire to make a meaningful contribution to society, influence their commitment to public sector roles (Wright, 2003). Further, performing altruistic acts or receiving intrinsic rewards are regarded as compensation for the low levels of extrinsic reward associated with the sector (Wright, 2001). Perry and Wise (1990) define this commitment as Public Sector Motivation (PSM) and describe it as an individual's predisposition to respond to motives primarily or uniquely grounded in public institutions and organisations. At an organisational level, this manifests as affective 
commitment and motivates employees to remain within the organisation (Kelloway \& Barling, 2000). At an inter-organisational level, the contribution that the collaboration makes to the wider population (i.e. society) assists team members to overcome potential issues and to participate in the collaboration based on its contribution in the bigger picture.

Camilleri (2007) purports that PSM is influenced by the organisational environment, such that team members must be supported through clear articulation of expectations. Further, employees require concise, unequivocal goals that must be clearly prioritised in instances where there may be instances of conflicting or competing goals (Camilleri, 2007). In an inter-organisational context, this need becomes increasingly important due to the complexity of the environment in which each organisation may have individual goals (in addition to the collaboration goal).

\subsubsection{Perceived ease of use \& perceived usefulness of ICT tools}

As noted in the previous chapter, the technology acceptance model (TAM) shows that the perceived ease of use of technology and perceived usefulness of technology can determine an individual's behavioural intention, and subsequent actual use of ICT tools (Davis \& Venkatesh, 1996). Though this study did not set out to test the TAM model, participants' observations about technology and reasons for low levels of interaction with technology clearly align with findings associated with the TAM model. Participants recounted difficulties logging onto the workspace, in navigating the site, and in accessing information held on the workspace. These difficulties resulted in their belief that the shared workspace was not an easy tool to use. As a consequence, participants were reluctant to engage with the tool, despite the fact that in some instances, they had not had direct experience with the tool. Rather, they based their opinions on anecdotal evidence derived from the experiences of others. As a result, not only was the shared workspace perceived as difficult to use, but it was regarded as lacking a useful purpose thus affecting its perceived usefulness.

\subsubsection{Individual technical ability}

Individuals' level of technical ability also influenced ICT use. Syed-Ikhsan \& Rowland (2004) identify this factor as "technology know-how", and posit that adequate 
technology training has a positive relationship with the creation and transfer of knowledge. Certainly the reported experiences of some participants indicated that a lack of adequate training negatively impacted their actual use and perceived usefulness of the technology available (see Case 3). Thus, individual technical ability, and a lack of training are underlying aspects that influence individuals' use of ICT tools.

Kim \& Lee (2006) suggest that the public sector may benefit from the development of more user-friendly ICT tools. This seems an appropriate suggestion when considering the difficulties reported by participants in regard to shared workspaces. However, this must be accompanied by adequate levels of training that engenders increased confidence in individuals' perceived and actual ICT ability.

\subsection{Contributions and Implications}

The research contributes to, and has implications for, both researchers and practitioners.

\subsubsection{Research contributions and implications}

This research stemmed from the identification of a number of gaps in the extant literature. Notably, the literature review revealed limited research relating to the practice of knowledge sharing within the public sector, and that even less had been undertaken at an inter-organisational level. In addition, much of the knowledge sharing literature derives from the technology transfer literature (Tang, 2008) resulting in a lack of empirical studies that explore knowledge sharing from a social capital perspective. This latter gap is particularly accentuated in the public sector context. Primarily, this study contributes to the small, but growing, number of studies (see Ismael \& Yusof, 2010; and Luna-Reyes \& Garcia, 2008) that investigate knowledge sharing in the public sector context. While the importance of knowledge sharing to the sector has been recognised for some time (OECD, 2003; Willem \& Buelens, 2007), this study represents one of only a few that investigate the phenomenon in the public sector context.

The study has also contributed to the bodies of research relating to both ICT and social capital and their relationship to knowledge sharing. The study has provided important clarification and greater understanding of the role that ICT plays in public sector 
knowledge sharing. While, previous studies have debated whether ICT is a driver or an enabler of knowledge sharing, the overall premise of prior research has still positioned ICT as an important factor. This research found a substantial gap between the importance of ICT from a sector perspective, and its actual use and importance to participants engaged in inter-organisational sharing at a grassroots level. While the sector sees ICT as an important factor in supporting knowledge sharing, participants do not. The study clearly establishes that, from an information-based perspective, ICT enables the central storage of documented information and is an important aspect of information sharing. However, it concludes that ICT does not play a role in determining whether personal knowledge, in the form of individual experience and expertise, will be shared - this latter decision is based on SC related factors. This has important implications for future studies and it is suggested that future ICT related research focuses on the relationship between the factors found to influence ICT availability and use (i.e. leadership, perceived use and usefulness of technology, and individual technical ability). Better understanding of these factors will support the development of ICT tools that are most appropriate for, and will be better utilised, in interorganisational collaboration.

The study has also shown the importance of the relationship between social capital and knowledge sharing in the public sector context, and has established social capital as a key theoretical perspective that requires further insight and investigation. The study identified that factors such as trust, culture, and shared purpose are important aspects of public sector-based knowledge sharing, and validated the need for better exploration of $\mathrm{SC}$ issues in the public sector context. Previously, social capital research in the public sector context has been limited to its role in civic terms. This research provokes greater interest in SC as a key determinant of knowledge sharing and concludes that increased research emphasis on aspects of SC has the potential to improve theoretical understandings of knowledge sharing.

The development of the conceptual framework addresses the central theoretical purpose of this research by expanding and deepening understanding of inter-organisational knowledge sharing and provides a major contribution to researchers engaged in knowledge sharing studies. Although, several knowledge sharing frameworks were 
identified during the literature review (see Ipe 2003; Lee \& Al-Hawamdeh, 2002; Smith $\&$ McKeen, 2003), none of these models related specifically to the public sector context, or had been derived from empirical evidence gathered from instance of interorganisational collaboration in the public sector. Ismael \& Yusof (2010) question the fit between existing knowledge sharing models and the public sector. The framework resulting from this research is based on establishing theory pertaining to the private sector, but has provided specific, contextualised findings, based solely on public sector based empirical evidence. The framework therefore bridges a key gap in the existing research and contributes to the development and growth of research aimed specifically at the public sector. The framework may be used by researchers in its entirety, or in part, to further validate the phenomenon in the specific contextual environment.

The discovery of the six key antecedents pertaining to knowledge sharing in the public sector provides a key research contribution. These antecedents influenced the development of social capital within the inter-organisational teams, and also played a role in determining the availability and use of ICT. In particular, the identification of "the intrinsic value of the work" as a key motivational factor in public sector interorganisational knowledge sharing provides substantive evidence to support the claims of some researchers who argue that knowledge sharing differs between the public and private sectors. This factor was found to be the most influential factor in terms of motivating individuals to share knowledge, and is very specific to the public sector. Thus, it is reasonable to conclude that, as noted above, frameworks derived from private sector based research do not wholly fit the public sector context. To date, research into this factor, coined by Perry \& Wise (1990) as Public Sector Motivation, has been confined to the role that PSM plays in retaining and motivating participation in the public sector. This study shows that PSM also plays a critical role in supporting and motivating knowledge sharing between individuals engaged in inter-organisational sharing. This constitutes an important finding for future research with the potential to change the way that knowledge sharing research is approached within the sector.

The use of SNA was an additional and valuable aspect of the research. It revealed characteristics about the inter-organisational teams' networks that otherwise would not have emerged. For example, the important role of information intermediaries was 
identified solely through analysis of the SNA data (see Chapter 8, p. 276). These individuals play an important role in assisting information and knowledge flows between team leaders, but this did not emerge through the qualitative research phases of the study. Thus, the identification of these information intermediaries is an important research finding that can contribute to future studies. The use of SNA in future studies will help to further reveal the role of the information intermediary and to better understand their value in inter-organisational teams.

The study confirms the validity and value of SNA as a research method within the public sector context, and at the inter-organisational level. Though social network analysis has been used extensively to explore knowledge sharing networks in organisations (Cross, Parker \& Sasson 2003; Kilduff \& Tsai, 2003), there has been very little application within the public sector, and its use in inter-organisational studies, though apparent (see Mead 2001; Hansen et al., 2002) is not extensive. In this study, by providing unique visual representations of the networks, supported by strong quantitative measures, SNA provided clear evidence about how knowledge was "actually" shared within the inter-organisational teams. It also enabled the researcher to validate aspects of social capital, such as trust, and understand how these were reflected within the actual interactions between individuals. Thus, by providing visual and quantitative representations of how knowledge is actually shared within interorganisational teams, SNA constitutes an important tool that can enable researchers to better understand the complex nature of inter-organisational sharing.

Historically, the majority of studies that investigate knowledge sharing have adopted a single research method approach. However, the use of multi-phased, multi-method, multiple-case research was found to be particularly valuable in supporting this study. The approach enabled the researcher to gain a deep, meaningful understanding of the research results yet allowed a greater ability to generalise the results due to the creation of multiple data sets, and greater degree of data triangulation. The approach adopted in this study has also enabled the identification of contradictions between the data gathered using different methods; this would be unlikely in a study using a single research method. Thus, the application of mixed methods research, and a sequential approach to data collection and analysis, offers researchers an increased opportunity to identify 
and investigate the phenomenon, to identify contradictions, and to provide greater insight into aspects of the research that might otherwise go unchallenged. Though this type of approach does require additional time, and adds complexity to the analysis and synthesis of research findings, it provides significant benefits to the researcher.

\subsubsection{Practitioner contributions and implications}

This research also has contributions and implications that can assist practitioners working in the practice of inter-organisational knowledge sharing.

At a sectoral level, this study identifies important aspects of inter-organisational collaboration that can assist governments in their ongoing endeavours to progress joined-up-government. Knowledge sharing has traditionally been a problematic area for the public sector (OECD, 2003). This study provides a singular insight into the complexities of knowledge sharing in the public sector gained through the perspectives of individuals at the heart of inter-organisational knowledge sharing.

The study provided significant insight into the use of ICT tools in inter-organisational collaboration. While the benefits of ICT are recognised in terms of information storage and integration, the study has shown that tacit knowledge sharing will be more readily facilitated through a focus on SC issues such as building trust between team members, and focussing on developing a sense of group identity and shared purpose. The research also shows that there is a fundamental gap between perceptions about ICT at strategic and operational levels. At the strategic level, the e-government framework proposes ICT tools, such as shared workspaces, as key collaborative tools, yet their actual availability and use at an operational level (i.e. within the Cases) is very limited. This has important consequences for the sector's development and use of ICT and provides key understandings about how inter-organisational teams perceive and use ICT tools.

The development of the conceptual framework, together with the detailed study findings, provide an integrated framework that may be used by practitioners to support and guide inter-organisational knowledge sharing in the public sector context. The framework can be used as a blue-print, or check-list, in considering aspects of the 
collaboration. For example, prior to the commencement of inter-organisational collaboration, specific emphasis should be given to maximising social capital development through deliberate consideration regarding the collaboration design and leadership. While projects such as Review of the Centre, Pathfinder and the governments Key Development Goals (see p. 64-65), have made recommendations regarding inter-organisational collaboration, to date none have identified the specific role of SC and the important part this plays in influencing knowledge sharing at the inter-organisational level. In addition, early attention to the six antecedents identified within the framework will help organisations and inter-organisational team leaders to maximise the opportunities for knowledge sharing to occur.

Finally, the sector has the potential to establish extensive informal knowledge networks as a result of individuals' participation in inter-organisational collaborative endeavours. The establishment of extended networks afford individuals the opportunity to develop their own experience and expertise through interactions with individuals with a diverse range of knowledge sets. Further, the building of these informal networks enables the establishment of weak ties which allows greater and quicker access to these knowledge sets by reducing the distance between actors. These networks also have the potential to improve inter-organisational collaboration. As instances of collaborations increase, it is likely that individuals will have increased opportunities to engage with others on multiple occasions, thus enabling the further embedding of SC stocks at an individual and sectoral level. As familiarity grows between individuals, issues such as trust, identity, and shared culture should become less problematic.

\subsection{Limitations}

The study has certain limitations that need to be taken into account when considering the study and its contributions. In most instances, these limitations were identified at the outset of the research, and where possible, the research methodology was strengthened and each phase of the study rigorously planned to limit the impacts of the issues. 


\subsubsection{Limitations of case research}

While case research is identified as appropriate for exploratory research (Creswell, 2003) and a suitable approach for investigating a contemporary phenomenon through a real-life context (Yin, 1989), case research is also subject to criticism regarding the ability to generalise from the case study findings from the sample to a general population (Lee, 1989; Tellis, 1997). To counter this limitation, the researcher made several fundamental design decisions. Firstly, the research employs multiple case studies, which several scholars identify as an important factor in supporting generalisability of a study (Benbasat et al., 1987; Galliers, 1992; Miles \& Huberman, 1994; Ragin, 1994; Yin 2005). Secondly, the study employs multiple research methods including one-on-one unstructured interviews, data gathering through a survey instrument, and the use of social network analysis. Such an approach enables the generation of multiple data sets (Sawyer, 2001) resulting in research that is more difficult to contest than single method research (Petter \& Gallivan, 2004). In addition, member checking was undertaken by forwarding interview transcripts to the interviewee for review.

\subsubsection{Limitations of social network analysis}

The interpretation of findings derived from social network data can be limited by two key aspects (Borgatti \& Molina, 2003; Cross \& Parker, 2004).

\section{Participant dropout}

Firstly, findings derived from SNA data can be negatively affected if some participants opt out of the study and this can lead to important gaps in the resulting interpretation of the network analysis. For example, key relationships may be omitted from the data. In order to counter this possibility, the nature of the process of engagement with research Cases helped ensure that opportunities for individual participants not to participate were limited. In the three instances where this did occur, discussion was undertaken with the team leaders to determine how significant this would be in terms of gaining a full understanding of the collaboration. As a result, their names were still included on the SNA questionnaire but a decision was taken to remove the individual from all aspects of 
the research. Subsequent completion of the questionnaires identified only two instances where these individuals had been identified by others. While their omission still constitutes a limitation and is included here for completeness reasons, their omission was not likely to significantly limit the interpretation of the findings.

\section{Accuracy of self-reported data}

As with other research methods, a further potential limitation of SNA research concerns the accuracy of self-reported data (Wasserman \& Faust, 1994). The two main reasons for inaccuracy are due to individuals simply forgetting information that may be relevant, and more deliberate omissions. This latter category is of particular relevance in social network analysis, where individuals are asked to contribute information about their relationships with other participants which can often be deemed to be sensitive and as such, can evoke defensiveness. In this study, this risk may also be accentuated by the general risk-averse nature of public sector organisations. As the study showed, participants were sometimes reluctant to share knowledge with other team members, so clearly might be reluctant to share with a complete stranger. To limit the potential impact of inaccurate data, the researcher employed multiple research methods. The generation of multiple data-sets enabled the researcher to compare and contrast the independent sets of results and to identify and follow-up evidentiary contradictions. In addition, the final phase of the research provided the researcher with opportunities to explore the self-reported data through face-to-face interaction with several participants. This method of interaction was found to be most effective in building trust with participants and consequently being able to explore issues more frankly.

\subsubsection{Other limitations}

The final limitations concern issues associated with identifying and securing research Cases, and the researcher's subsequent access to participants. As noted above, the literature review identified that the lack of empirical evidence in the public sector might be due to the sector's sensitivity to media and public scrutiny and its associated risk (Taylor \& Wright, 2004). Therefore, allowing a researcher access to interorganisational collaborations might also be perceived as a potential risk. As detailed in Chapter 3 (p. 101), several organisations declined to participate and a number of 
organisations did not respond to the initial research request. As a consequence, the final selection of research Cases was made from the small number of organisations that indicated interest in participating. Access to further research sites may have extended the findings or provided different perspectives. However, while this potential limitation is acknowledged, the use of multiple cases increased the opportunities for generalising the study findings, and the identification of patterns of similar behaviour among Case participants suggests that the Cases are representative of inter-organisational collaboration within the sector.

A further limitation relates to interaction between the researcher and Case participants and the limitations (above) in relation to building trust with participants, and addressing the accuracy of self-reported data. As documented in the Case studies, the nature of inter-organisational collaboration often puts considerable pressure on individuals' time. This factor, together with the geographical distribution of participants restricted the degree of researcher interaction with some participants. As a result, in Case 4, for example, the researcher was unable to gather interview data from representatives from all participating organisations. This may have resulted in the overall interpretation of data being somewhat biased towards the organisations whose participants were engaged with on a face-to-face basis. Although the researcher did take steps to counter this bias (for example, by telephoning individuals in organisations who could not be interviewed on a face-to-face basis), the data collected from these interactions was not as rich, or detailed as that gathered in face-to-face interactions.

The limitations of this study have been systematically identified and, where possible, mitigation strategies have been implemented to reduce the effect of the limitations on the study findings. It is considered that they do not detract from the overall interpretation and significance of the findings, but may serve as future research avenues.

\subsection{Future research opportunities}

This thesis culminates with the identification of future research opportunities arising from this study. The multi-faceted nature of the research means that there are many avenues of opportunity for future research. These include: confirmation of the study findings through the exploration of specific research factors in more detail and depth; 
application of the conceptual framework to other Cases; or further application of social network analysis within collaborative ventures.

\subsubsection{Confirmation of the research findings}

The first opportunity lies in confirming the findings of this study. The research was conducted through a multiple case approach and case selection was undertaken using a literal replication strategy (Yin, 1989). Though this approach enhances the generalisability of the study, there would be value in exploring the findings in other instances of inter-organisational collaboration. The research points to the existence of relationships and inter-dependencies between several of the factors explored in the research. Future research across a wider number of collaborative ventures would help clarify the validity of these relationships and add extra depth to the findings. In future studies, researchers may wish to expand the number of one-on-one interviews to ensure that all organisations have an opportunity to provide further depth and perspective to the findings.

\subsubsection{Focus on individual aspects of the research}

The second opportunity lies in exploring specific aspects of the research in greater depth. The nature of the primary research objective (to acquire a better understanding of knowledge sharing within the public sector, inter-organisational context) required the researcher to explore a broad range of factors and required extensive time in both data collection and analysis. Findings indicate several factors that could form the basis of more in-depth study. For example, collaboration design was identified as a key antecedent that had strong influence on the development of trust, agreeing on the shared purpose, and establishing a group identity. Therefore a better understanding of this single factor would be advantageous in assisting inter-organisational teams to facilitate knowledge sharing.

Focussing on individual aspects of SC, for example trust, would also enable deeper exploration of the links between the qualitative and SNA datasets. 


\subsubsection{Application of the conceptual framework}

The third opportunity arises from the application and testing of the conceptual framework. The multi-dimensional nature of the framework lends itself to either application of the entire framework, or application of a single or number of dimensions. Either approach would result in the development of a substantial body of data that would allow for systematic comparisons between Cases to be carried out.

In this research, the conceptual framework has evolved from a basic model encompassing the key aspects of the research to a framework that identifies relationships between these aspects. However, the research does not extend to identifying cause and effect. Future application of the framework could be used to examine causality between individual factors of the research.

\subsubsection{Application of social network analysis}

In this study, social network analysis enabled the researcher to compare the formal and informal collaboration structures, to identify information flows within the network, and to better understand the roles that each team member played. Future exploration of inter-organisational collaboration could focus on one or several network aspects. For example, the researcher could focus more closely on understanding the factors that contribute to the strength of ties between team members. Or, the researcher may wish to better understand specific roles within the network. This might be undertaken by expanding the range of questions at a network level, and be followed up with one-onone interviews with those identified as playing specific roles within the network. The application of social network analysis to collaborative ventures that are identified as problematic may also provide organisations with a better understanding of the specific dynamics of the inter-organisational network, thus enabling the identification of specific interventions.

\subsubsection{Extending the scope of the research}

While this study focused on knowledge sharing between public sector organisations, collaboration also occurs between public and private sector organisations. Future research might be extended to encompass other types of collaborative arrangement, such 
as public-private, or between governments of different countries. This latter area would be of significant benefit as international boundaries become more blurred through increasing collaboration between countries such as New Zealand its close neighbour, Australia. Such an extension of the research would broaden the findings relating to cognitive social capital areas, such as shared culture and identity, from organisational to national levels.

\subsection{Summary of Chapter 9}

This chapter concludes the research. The chapter began by reviewing the nature of the research and its key findings. It re-introduced the conceptual framework developed at the outset of the study, and updated the framework to incorporate the study findings. The implications and contributions of these findings were then discussed in regard to both the research and practitioner communities. The chapter identified the limitations of the study and concluded by identifying the opportunities that arise from this study in relation to further research.

This study has highlighted the increasing importance of inter-organisational knowledge sharing in the public sector, but has also identified this as an area in which little is yet known. The study has provided some insight into the knowledge sharing behaviours of inter-organisational teams but there remains a significant concomitant need for researchers to develop and extend studies into this phenomenon. 


\section{REFERENCES}

Abrams, L. C., Cross, R., Lesser, E., \& Levin, D. (2003). Nurturing interpersonal trust in knowledge-shaving networks. Academy of Management Executive, 17(4), 64-77.

Ackerman, M. S., \& Halverson, C. (2004). Sharing expertise: The next step for knowledge management. In M. Huysman \& V. Wulf (Eds.), Social capital and information technology (pp. 273-299). Massachusetts Institute of Technology, Cambridge.

Adler, P., \& Kwon, S. W. (2002). Social capital: Prospects for a new concept. Academy of Management Review, 27(1), 17-40.

Alam, A., Abdullah, Z., Ishak, N.A., \& Zain, Z.M. (2009). Assessing Knowledge Sharing Behaviour among Employees in SMEs: An Empirical Study. International Business Research 2(2), 115-122.

Alavi, M., \& Leidner, D. (1999). Knowledge Management Systems: Emerging Views and Practices from the Field. Proceedings of the $32^{\text {nd }}$ Hawaii International Conference on System Sciences (HICSS-32), Maui, Hawaii (pp.1-11).

Alavi, M., \& Leidner, D. (2001). Knowledge management and knowledge management systems: Conceptual foundations and research issues. MIS Quarterly, 25(1), 107 - 136.

Aldridge, S., Halpern, D., \& Fitzpatrick, S. (2002). Social capital: A discussion paper. Cabinet Office, Performance and Innovation Unit. Retrieved from http://www.strategy.gov.uk/2001/futures/attachments/socialcapital/pdf

Al-Alawi, A.I., Al-Marzooqi, N.Y., and Mohammed, Y.F. (2007). Organizational Culture and Knowledge Sharing: Critical Success Factors. Journal of Knowledge Management, $11(2), 22-42$.

Ancona, D. (1990). Outward bound: Strategies for team survival in an organization. Academy of Management Journal, 33, 334-365.

Ancona, D.G., \& Caldwell, D.F. (1992), Bridging the boundary: external process and performance in organisational teams. Administrative Science Quarterly, 3, 634-65.

Anklam, P. (2003). KM and the Social Network. Knowledge Management Magazine.

Argote, L.(1999). Organizational learning: Creating, retaining and transferring knowledge: Kluwer Academic.

Argote, L., McEvily, B., \& Reagans, R. (2003). Managing Knowledge in Organizations: An Integrative Framework and Review of Emerging Themes. Management Science 49(4), 571-582.

Ashforth, Blake E., \& Mael, Fred (1989). Social Identity Theory and the Organisation. Academy of Management Review, 40(1), 20-39.

Badaracco, J.L. (1991). The knowledge link. Boston: Harvard Business School Press

Baehler, K. (2001). Intervention Logic: A presentation to the Pathfinder Project. Pathfinder Workshop presentation. Retrieved from http://io.ssc.govt.nz/pathfinder/documents/pathfinder-BB3-intervention_logic.pdf

Banks, S., \& Powell, A. (2002). Developing Institutional Readiness for Implementing Networked Learning, Networked Learning. Proceedings of the 3rd International Conference. Sheffield: Sheffield University.

Bartol, K., \& Srivastava, A. (2002). Encouraging knowledge sharing. Journal of Leadership \& Organizational Studies, 9(1), 64-76. 
Bassey, M. (1990), On the Nature of Research in Education, Faculty of Education, Nottingham Polytechnic.

Bate, S.P. \& Robert, G. (2002). Knowledge management and communities of practice in the private sector: Lessons for modernizing the National Health Service in England and Wales. Public Administration 80(4), 643 - 663.

Benbasat, I., Goldstein, D., \& Mead, M. (1987). The case research strategy in studies of information systems. MIS Quarterly, 11(3), 368 -386.

Bera, P., \& Wand, Y. (2004). Analyzing OWL using a Philosophy-Based Ontology.

Proceedings of International Conference on Formal Ontology in Information Systems (FOIS 2004). Torino (Italy), IOS press, pp. 353-362.

Blackler, F. (1995). Information Space: A Framework for Learning in Organizations, Institutions and Culture. London: Blackwell.

Bloodgood, J.M \& Salisbury, W.D. (2001). Understanding the influence of organisational change strategies on information technology and knowledge management strategies. Decision Support Systems. 31, 55-69.

Bock, G.W. \& Kim, Y-G. (2002). Breaking the myths of rewards: An exploratory study of attitudes about knowledge sharing. Information Resources Management Journal, 15(2), 14-21.

Bock, G.W., Zmud, R.W., \& Lee, J. N. (2005). Behavioral Intention Formation in Knowledge Sharing. MIS Quarterly, 29,1, 87-111

Bollinger, A. S and Smith, R.D. (2001). Managing Organizational Knowledge as a Strategic Asset. Journal of Knowledge Management, 5,1, 8-18.

Bonoma, T. (1985). Case research in marketing: opportunities, problems, and a process. Journal of Marketing Research, 12, 199-208.

Borgatti, S. P., Jones, C., \& Everett, M., (1998). Network measures of social capital. Connections, 22(2), 36-44.

Borgatti, S. P. \& Cross, R. (2003). A relational view of information seeking and learning in social networks. Management Science, 49(4), 432-445.

Borgatti S.P. \& Foster, P.C. (2003). The Network Paradigm in Organizational Research: A Review and Typology. Journal of Management, 29(6), 991-1013.

Borgatti, S., P., \& Molina, J., Luis. (2003). Ethical and strategic issues in organizational social network analysis. The Journal of Applied Behavioural Science, 39(3), 337-349.

Borkan, J. (2004). Mixed methods studies: A foundation for primary care research. Annals of Family Medicine, 2(1), 4-6.

Bosua, R. \& Scheepers, R. (2007). Towards a model to explain knowledge sharing in complex organizational environments. Knowledge Management Research and Practice, 5, 93109.

Bourdieu, P. (1985) Social Space and the Genesis of Social Groups. Theory and Society, 14(6).

Braddon, D. and Foster, D. (eds) (1996), Privatisation: Social science themes and perspectives. Aldershot, Dartmouth.

Brass, D. J. (1995). A social network perspective on human resource management. Research in Personnel and Human Resource Management, 13, 39-79. 
Brown, J.S. \& Duguid, P. (1998). Organizing knowledge. California Management Review. 40(3), (1998), 90-111.

Brown, K., Ryan, N., \& Parker, R. (2000). New modes of service delivery in the public sector : Commercialising government services. The International Journal of Public Sector Management, 13(3), 206-221.

Bullock, R., Mountford, H. \& Stanley, R. (2001). Better Policy Making. Retrieved from http://www.civilservant.org.uk/betterpolicymaking.pdf.

Burt, R.S. (1992). Structural Holes. Harvard University Press. Cambridge, MA.

Carrington, P. J., Scott, J., \& Wasserman, S. (2005). Models and Methods in Social Network Analysis. Cambridge, Cambridge University Press.

Castells, M. (1996) The Rise of the Network Society. Oxford: Blackwell.

Chen, W., \& Hirscheim, R. (2004). A paradigmatic and methodological examination of information systems research from 1991 to 2001. Information Systems Journal, 14, 197235.

Chen, C-M., Lin, T.C., Liou, Y.C., \& Liu, Y.C. (2009). Social capital, knowledge based view and entrepreneurial opportunity. International Journal of Management and Enterprise Development, 7(2), 163-182.

Cheng, J-H., Yeh, C-H., \& Tu, C-W. (2008). Trust and knowledge sharing in green supply chain. Supply Chain Management: An International Journal, 13(4), 283-295.

Choo, C.W. (1998). The Knowing Organization: How Organizations Use Information to Construct Meaning, Create Knowledge, and Make Decisions. New York: Oxford University Press.

Chow, W.S., \& Chan, L.S. (2008). Social network, social trust and shared goals in organizational knowledge sharing. Information \& Management, 45, 458-462.

Chua, A. (2001). Relationship between the types of knowledge shared and the types of communication channels used. Journal of Knowledge Management Practice, 2. Retrieved from: http:www.tlainc.com/jkmp2.html.

Chua, A. (2002). The influence of social interaction on knowledge creation. Journal of Intellectual Capital, 3(4), 275-392.

Clark, K., \& Staunton, N. (1989). Innovation in technology and organization. London: Routledge.

Clegg, S., Hardy, C., \& Nord, W. R. (1996). Handbook of organization studies. London: Sage.

Cohen, D., \& Prusak, L. (2001). In good company: How social capital makes organizations work. Boston: Harvard Business School.

Coleman, J.S. (1990). Foundations of Social Theory. Cambridge, MA: Harvard University Press.

Cong, X., \& Pandya, K. (2003). Issues of knowledge management in the public sector. Electronic Journal of Knowledge Management, 1(2), 25-33.

Connell, N., Klein, J., \& Powell, P. (2003). It's tacit knowledge but not as we know it: Redirecting the search for knowledge. Journal of the Operational Research Society, 54, 140-152.

Connelly, C. (2000). Predictors of Knowledge Sharing in Organisations. (Masters Thesis, Queen's University, Ontario, Canada). Retrieved from: http://www.business.mcmaster.ca/hrlr/profs/connell/thesis.pdf. 
Connelly, C., \& Kelloway, E. (2003). Predictors of employees' perceptions of knowledge sharing cultures. Leadership \& Organization Development Journal, 24(5), 294-301.

Courtney, Jr., J.F., Haynes. J. and Paradice, D.B. (eds.) (2005). Inquiring Organizations: Moving from Knowledge Management to Wisdom, Hershey: Idea Group Publishing,.

Cowles, D. (1997). The role of trust in customer relationships: asking the right questions. Management Decision 34(4), 273-282.

Cresswell, A. M., Pardo, T. A., Thompson, F., Canestraro, D. S., Cook, M., Black, L. J., LunaReyes, L. F., Martínez-Moyano, I. J., Andersen, D. F., \& P., R. G. (2002). Modeling Intergovernmental Collaboration: A System Dynamics Approach. Proceedings of the $35^{\text {th }}$ Annual Hawaiian International Conference on System Sciences, (HICSS-02), 3, $96 \mathrm{~b}$.

Creswell, J. W. (2003). Research design: Qualitative, quantitative, and mixed methods approaches. Sage: Thousand Oaks.

Cross, R,. \& Baird, L. (2000). Technology is not enough: Improving Performance by Building Organizational Memory. Sloan Management Review. 41(3), 41-54.

Cross, R. \& Parker, A. (2004). The Hidden Power of Social Networks: Understanding How Work Really Gets Done in Organizations. Harvard Business School Press.

Cross, R., Nohria, N., \& Parker, A. (2002). Six myths about informal networks - and how to overcome them. MIT Sloan Management Review, 43(3), 67-75.

Cross, R., Parker, A., Prusak, L., \& Borgatti, S. P. (2001). Knowing what we know. Supporting knowledge creation and sharing in social networks. Organizational Dynamics, 30, 100120.

Cross, R., \& Prusak, L. (2002). The people who make organizations go - or stop. Harvard Business Review, 80(6), 104-112.

Crotty, M. (1998). The Foundations of Social Research: Meaning and Perspective in the Research Process. London: SAGE Publications Ltd.

Cummings, J. (2003). Knowledge sharing: A review of the literature for the World Bank Operations Evaluation Unit. Retrieved from: http://lnweb90.worldbank.org/oed/oeddoclib.nsf/DocUNIDViewForJavaSearch/D9E38 9E7414BE9DE85256DC600572CA0/\$file/knowledge_eval_literature_review.pdf.

D’Amour, D., Goulet, L., Labadie, J. F., Martin-Rodriguez, L., \& Pineautl, R. (2008) A model and typology of collaboration between professionals in healthcare organizations. $B M C$ Health Services Research, 8, 188.

Daft, R. L., \& Lengel, R.H. (1986). "Organizational Information Requirements, Media Richness and Structural Design." Management Science 32(5): 554-571.

Dalsgaard, C. (2006). Social software: E-learning beyond learning management systems. European Journal of Open, Distance and E-Learning, 2006/II. Retrieved from: http://www.eurodl.org/materials/contrib/2006/Christian_Dalsgaard.htm.

Darke, P., Shanks, G. \& Broadbent, M. (1998). Successfully completing Case Study Research: Combining Rigour, Relevance and Pragmatism, Information Systems Journal, 8, 273289.

Davenport, T. H. (1997). Information ecology. Oxford, UK: Oxford University Press.

Davenport, T. H., \& Prusak, L. (1998). Working knowledge. Boston: Harvard Business School Press. 
Davidson, C., \& Voss, P. (2003). Knowledge management: An introduction to creating competitive advantage from intellectual capital. Auckland: Tandem Press.

Davis, F. D. (1989). Perceived usefulness, perceived ease of use, and user acceptance of information technology. MIS Quarterly, 13(3), 319-340.

Davis, F. D., Bagozzi, R. P., \& Warshaw, P. R. (1989). User acceptance of computer technology: A comparison of two theoretical models. Management Science, 35, 9821003.

Dawes, S.S., \& Pardo, T. (2002). Building collaborative digital government systems: Systemic constraints and effective practices. In W. McIver \& A.K. Elmagarmid (Eds.), Advances in digital government technology, human factors, and policy (pp. 259 - 273). Norwell, MA: Kluwer.

Deane, R. (1986). Public sector reform: A review of the issues. In M. Clark \& E. Sinclair (Eds.), Purpose performance and profit: Redefining the public sector. Wellington: New Zealand Institute of Public Administration.

De Long, D., \& Fahey, L. (2000). Diagnosing Cultural Barriers to Knowledge Management. Academy of Management Executive, 14(4), 113-127.

Dixon, N. M. (2000). Common knowledge: How companies thrive by sharing what they know. Boston, Mass: Harvard Business School Press.

Dubé, L., \& Paré, G. (2003) Rigor in Information Systems Positivist Case Research: Current Practices, Trends, and Recommendations, MIS Quarterly 27(4), 597-636.

Durland, M. (2003). Understanding Sociograms: A Guide to Understanding Network Analysis Mapping. $\quad$ Retrieved from: http: //www.durlandconsulting.com/images/pdfs/Understanding_maps_11_03a.pdf

Edwards, G. (2010). Mixed-Method Approaches to Social Network Analysis. ESRC National Centre for Research Methods. Retrieved from: http://eprints.ncrm.ac.uk/842/

Earl, M. (2001). Knowledge management strategies: Toward a taxonomy. Journal of Management Information Systems, 18(1), 215-233.

Easterby-Smith, M., Lyles, M.A., \& Tsang E.W.K. (2008). Inter-Organizational Knowledge Transfer: Current Themes and Future Prospects. Journal of Management Studies, 45(4) 677-690.

Edwards, J. S., Handzic, M., Carlsson, S., \& Nissen, M. (2003). Knowledge management research \& practice: Visions and directions. Knowledge Management Research \& Practice, 1, 49-60.

E-government (2006). First Agencies sign up for GSN. Retrieved from: http://www.e.govt.nz/resources/news/2006/2006100501.html

E-government (2006a). E-governance in New Zealand. Retrieved from: http://www.it.iitb.ac.in/ prathabk/egovernancelegov_success_stories_nz.html

E-government (2009). Web 2.0 social networking tools. Retrieved from: http://www.e.govt.nz/resources/research/copy_of_progress/agencyinitiatives/chapter6.html/view?searchterm=social\%20network

Eisenhardt, K. M. (1989). Building theories from case study research, Academy of Management Review, 14(4), 532 - 550.

Ericksen, J., \& Dyer, L. (2004). Right from the Start: Exploring the Effects of Early Team Events on Subsequent Project Team Development and Performance. Administrative Science Quarterly, 49(3), 438-471 
Erlandson, D.A., Harris, E. L., Skipper, B. L. \& Allen, S. D. (1993). Doing naturalistic inquiry: a guide to methods. London: Sage.

Fahey, L. \& Prusak, L., (1998). The eleven deadliest sins of knowledge management. California Management Review, 40(3), 265-276.

Fedor, D. B., Ghosh, S., Caldwell, S. D., Maurer, T., J., \& Singhal, V., R. (2003). The effects of knowledge management on team member's ratings of project success and impact. Decision Sciences, 34(3), 513-539.

Feldman, D. 1984. The development and enforcement of group norms. Academy of Management Journal, 9(1): 47-53.

Fowler, A. \& Pryke, J. (2003). Knowledge management in public service provision: the Child Support Agency. International Journal of Service Industry Management, 14(3), 254283.

Frederickson, H. G., \& Smith, K. B. S. (2003). The public administration theory primer. Boulder, CO: Westview Press.

Fu, S., and Lee, M. (2005). IT-based knowledge sharing and organisational trust: The development and initial test of a comprehensive model. Proceedings of the 2005 European Conference of Information Systems, Available: http://is2.lse.ac.uk/asp/aspecis/20050071.pdf, [28 July 2007].

Fukuyama, F. (1995). Trust: The social virtues and the creation of prosperity. New York: Free Press.

Fukuyama, F. (2000). The great disruption: Human nature and the reconstitution of social order. Bury St Edmunds, UK: St Edmundsbury Press.

Galliers, R. D. (1992). Choosing information systems research approaches. In R. D. Galliers (ed.), Information Systems Research: Issues, Methods and Practical Guidelines. Oxford: Blackwell Scientific.

Galliers, R., \& Newell, S. (2001). Electronic commerce and strategic change within organizations: Lessons from 2 cases. Journal of Global Information Management, 9, 315-322.

Garton, L., Haythornthwaite, C., \& Wellman, B. (1997). Studying online social networks. Journal of Computer Mediated Communication, 3(1).

Gavigan, J., Ottitsch, M. \& Mahroum, S. (1999). Knowledge and learning - towards a learning Europe. Proceedings of the 2000 IPTS Futures Project Conference. Retrieved from: http://futures.jrc.es/menupage-b.htm.

Goh, S. C. (2002). Managing effective knowledge transfer: An integrative framework and some practice implications. Journal of Knowledge Management, 6(1), 23-30.

Gold, A., Malhotra, A., \& Segars, A. (2001). Knowledge management: An organizational capabilities perspective. Journal of Management Information Systems, 19(1), 185-214.

Goldsmith, S. \& Eggars, W. D. (2004). Governing by Network The New Shape of the Public Sector. Brookings Institute Press \& John f Kennedy School Of Government At Harvard University

Granovetter, Mark S. (1973). The Strength of Weak Ties, American Journal of Sociology 78 (6), $1360-1380$.

Grant, R. M. (2002). Contemporary Strategy Analysis: Concepts, Techniques, Applications. Oxford, UK: Blackwell Publishing. 
Green, R. (2003). Measuring goodwill trust between groups of people: three years of an oil industry alliance. Strategic Change 12(7), $367-379$.

Greene, Jennifer C., Caracelli, Valerie J. \& Graham, Wendy F. (1989). Toward a conceptual framework for mixed-method evaluation design. Educational Evaluation and Policy Analysis, 11(3), 255-74.

Greveson, C.W., \& Damanpour, F. (2007). Performance implications of organisational structure and knowledge sharing in multinational R\&D networks. International Journal of Technology Management, 38(1-2), 113 - 136.

Groff, Todd, R. \& Jones, Thomas, P. (2003). Introduction to Knowledge Management: KM in Business. Burlington, MA: Butterworth - Heinemann.

Grover, V. \& Davenport , T.H. (2001). General Perspectives on Knowledge Management: Fostering a Research Agenda. Journal of Management Information Systems, 18(1), 521.

Gruenfeld, D.H., Mannix, E.A., Williams, K.Y., \& Neale, M.A. (1996). Group composition and decision-making: How member familiarity and information distribution affect process and performance. Organizational Behavior \& Decision Processes, 67(10), 1-15.

Guba, E. G., \& Lincoln, Y. S. (1994). Competing paradigms in qualitative research. In N. K. Denzin \& Y. S. Lincoln (Eds.), Handbook of qualitative research (pp. 105-117.). Thousand Oaks, CA: Sage Publications.

Gurteen, D. (1998). Knowledge, creativity and innovation. Journal of Knowledge Management, 2(1), 5-13.

Hackett, B. (2000). Beyond Knowledge Management: New Ways to Work and Learn. The Conference Board (Publication R-1262-W-14). Available URL: http://www.conferenceboard.org.

Hahn, J., Moon, J.Y., \& Zhang, C. (2008). Emergence of New Project Teams from Open Source Software Developer Networks: Impact of Prior Collaboration Ties. Information Systems Research, 19(3), 369 - 385

Hanneman, M. \& Riddle, M. (2005). Introduction to Social Network Methods. Riverside, CA: University of California, Riverside

Hansen, M. T., Nohria, N., \& Tierney, T. (1999). What's your strategy for managing knowledge? Harvard Business Review, 77(March-April), 106-116.

Hansen, M. (2002). Knowledge networks: Explaining effective knowledge sharing in multiunit companies. Organization Science, 13(3), 232-248.

Hansen, M. (1999). The search-transfer problem: The role of weak ties in sharing knowledge across organization subunits. Administrative Science Quarterly, 44(1), 82-111.

Harris, P. (2005). The myth of the exploding public sector. Retrieved at: http://www.psa.org.nz/library/psa/newsstories/publicsectorpeterharrispaper

Harryson, S. J., Dudkowski, R., \& Stern, A. (2008). Transformation networks in innovation alliances - the development of Volvo C70. Handbook of qualitative research, 45, 73058.

Hawe, P., \& Ghali, L. (2007). Use of social network analysis to map the social relationships of staff and teachers at school. Health Education Research, 23(1), 62-69.

Haythornthwaite, C. (1996). Social network analysis: An approach and technique for the study of information exchange. Library and Information Science Research, 18, 323-342. 
Haythornthwaite, C. (1999). Work and community in networked organizations. In Leen d'Haenens (Ed.). Cyberidentities: Canadian and European Presence in Cyberspace (pp. 135-145). Ottawa, Ont.: University of Ottawa Press.

Healy, T., Cote, S., Helliwell, J.F., \& Field. S. (2001). The Well-being of Nations: The role of human and social capital. Organization for Economic Co-operation and Development, Paris.

Hearn, G., \& Rooney, D. (2002). The future role of government in knowledge-based economies. Foresight, 4(6), 23-33.

Hedesstrom, T. \& Whitley, E. (2000). What is meant by tacit knowledge? Towards a Better Understanding of the Shape of Actions. Proceedings of the 8th ECIS Conference, (pp. 46-51), Vienna.

Henderson, R. M., \& Clark, K. B. (1990). Architecture Innovation: The Reconfiguration of Existing Product Technologies and the Failure of Established Firms. Administrative Science Quarterly, 35(1), 9-30.

Hendriks, P. (1999). Why share knowledge? The influence of ICT on the motivation for knowledge sharing. Knowledge and Process Management, 6(2), 91-100.

Hill, C. J. H., \& Lynn, L. E. J. (2005). Is hierarchical governance in decline? Evidence from empirical research. Journal of Public Administration Research and Theory, 15(2), 173196.

Hirschheim, R., \& Klein, H. K. (1989). Four paradigms of information systems development. Communications of the ACM, 32(10), 1199-1216.

Hoegl, M., Parboteeah, K, Praveen., \& Munson, Charles, L. (2003) Team-Level Antecedents of Individuals' Knowledge Networks. Decision Sciences, 34(4), 741-769.

Holsapple, C. W. (2004). (Ed.) Handbook on knowledge management (pp. 408-438). New York: Springer-Verlag.

Hsu, C-L., \& Lin, Judy. (2008). Acceptance of blog usage: The roles of technology acceptance, social influence and knowledge sharing motivation. Information and Management, 45(1), 65-74.

Huber, G. P. (1991). Organizational learning: The contributing processes and the literature. Organization Science, 2, 88-115.

Huber, G. P. (2001). Transfer of knowledge in knowledge management systems: Unexplored issues and suggest studies. European Journal of Information Systems, 10, 72-79.

Huczynski, A. (1989). Training designs for organizational change. Management Decision: Quarterly Review of Management Technology, 27(4), 162-167.

Huysman, M \& de Wit, D. (2004). Practices of managing knowledge sharing: towards a second wave of knowledge management, Knowledge and Process Management, 11(2), 81-92.

Huysman, M., \& Wulf, V. (Eds.). (2003). Social capital and information technology. Cambridge, MA: Massachussets Institute of Technology.

Huysman, M. \& Wulf, V. (2006) IT to support knowledge sharing in communities: Towards a social capital analysis. Journal of Information Technology, 21, 40-51.

Inkpen, A.C. \& Tsang, T.W.M. (2005). Social capital, networks and knowledge transfer. The Academy of Management Review 20(1), 146-165.

Ipe, M. (2003). Knowledge sharing in organisations: A conceptual framework. Human Resource Development Review, 2(4), 337-359 
Jacobs, E. \& Roodt, G. (2007). The development of a knowledge sharing construct to predict turnover intentions. Aslib Proceedings: New Information Perspectives, 59(3), 229-248

Johnson, David J. (2009). Managing Knowledge Networks. New York, USA: Cambridge University Press.

Jorgensen, B. (2004). Individual and organizational learning: A model for reform for public organizations. Foresight, 6(2), 91-103.

Jones, G.R., \& George, J.M., 1998. The experience and evolution of trust: Implications for cooperation and teamwork. The Academy of Management Review, 23, 531-546.

Kakabadse, N., K., Kakabadse, A., \& Kouzmin, A. (2003). Reviewing the knowledge management literature: Towards a taxonomy. Journal of Knowledge Management, 7(4), 75-91.

Kang, S-C., Morris, S. S., \& Snell, S. A. (2007). Relational archetypes, organizational learning, and value creation: extending the human resource architecture. Academy of Management Review, 32, 236-256.

Kaplan, B. \& Maxwell, J.A. (1994) Qualitative Research Methods for Evaluating Computer Information Systems. In J. G. Anderson, C. E. Aydin, \& Jay Stephen J. (Eds.), Evaluating health care information systems: Methods and applications (pp. 45-68). Thousand Oaks, CA: Sage Publications.

Katz, N., Lazer, D., Arrow, H., \& Contractor, N. (2005). The network perspective on small groups: Theory and research. In Poole, M. S. \& Hollingshead, A.B. (Eds.) Theories of Small Groups: Interdisciplinary Perspectives (pp.277-312). Newbury Park, CA: Sage.Keast, R., Mandell, Myrna P., Brown, K. \& Woolcock, G. (2004). Network Structures: Working Differently and Changing Expectations. Public Administration Review, 64(3), 363-371.

Kekale, T., Takala, J., \& Ajmal, M.M. (2009) Cultural Impacts on Knowledge Management and Learning in Project-Based Firms. Vine, 39(4), 339-352.

Kelloway, K. \& Barling, J. (2000). Knowledge work as organizational behaviour. International Journal of Management Reviews, 2(3), 287-304.

Kilduff, M. \& Tsai, W. (2003) Social Networks and Organizations. London, UK: Sage Publications.

Kim, B. (2001). Social constructivism. In M. Orey (Ed.), Emerging perspectives on learning, teaching, and technology. Available Website: http://www.coe.uga.edu/epltt/SocialConstructivism.htm

Kim, S., Lee, J. (2006). The impact of organizational context and information technology on employee knowledge sharing capabilities. Public Administration Review, 370-375.

Kim, H., Lee, J., \& Kim. S. (2008). Linking Local E-Government Development Stages to Collaboration Strategy. International Journal of Electronic Government Research, 4(3), 36-56.

Kotlarsky, J. \& Oshri, I. (2005). Social ties, knowledge sharing and successful collaboration in globally distributed system development projects. European Journal of Information Systems, 14, $37-48$.

Kramer, R, M. \& Tyler, T.R. (Eds.) (1996). Trust in organizations: Frontiers of theory and research. Thousand Oaks, CA: Sage Publications. 
Kramer, R. M., Hanna, B. A., Su, S., \& Wei, J. 2001. Collective identity, collective trust, and social capital: Linking group identification and group cooperation. In M. E. Turner (Ed.), Groups at work: Theory and research. (pp. 173-196). Mahwah, NJ: Erlbaum.

Krishna, A. \& Schrader, E. (2002). The social capital assessment tool. In C. Grootaert \& T. v. Bastelaer (Eds.), Understanding and measuring social capital (pp. 17-44). Washington, DC: International Bank for Reconstruction and Developments.

Kwok, S.H., \& Gao, S. (2005). Attitude Toward Knowledge Sharing Behavior. The Journal of Computer Information Systems; 46(2), 45-51.

Lam, A \& Lambermont-Ford (2008). Knowledge Creation and Sharing in Organisational Contexts: A Motivation-Based Perspective. The School of Management, Royal Holloway University of London

Lang, J.C. (2004). Social context and social capital as enablers of knowledge integration. Journal of Knowledge Management, 8, 89-105.

Lee, A.S. (1989). A Scientific Methodology for MIS Case Studies, MIS Quarterly 13(1), 33-52.

Lee, L., \& Al-Hawamdeh, S. (2002). Factors impacting knowledge sharing. Journal of Information and Knowledge Management, 1(1), 49-56.

Lertpittayapoom, N., Paul, S., \& Mykytyn, P. (2007). A Theoretical Perspective on Effective Interorganizational Knowledge. Sharing Proceedings of the 40th Hawaii International Conference on System Sciences - 2007. Hawaii.

Lesser, E., \& Prusak, L. (2003). Creating value with knowledge: Insights from the ibm institute for knowledge-based organizations. New York.

Lewicki, R., McAllister, D., \& Bies, R. (1998). Trust and distrust: New relationships and realities. Academy of Management Review, 23(3), 438-458.

Lin, N. (2001). Social capital: A theory of social structure and action. Cambridge: Cambridge University Press.

Lincoln, Y.S. \& Guba, E.G. (1985). Naturalistic inquiry. Beverly Hills, CA: Sage.

Lipnack, J. \& Stamps, J. (1997). Virtual Teams: Reaching across space, time, and organizations with technology. John Wiley \& Sons, Inc.

Lips, A. M. B. (2008) Before, After or During the Reforms? Towards Information Age Government in New Zealand. Policy Quarterly, 4(2), 21-26.

Liu, A.Q., \& Besser, T. (2003). Social capital and participation in community improvement activities by elderly residents in small towns and rural communities. Rural Sociology 68, 343-365.

Lofstrom, M. (2009). Inter-organizational collaboration projects in the public sector: a balance between integration and demarcation. International Journal of Health Planning and Management, 25, 136-155

Luen, T. \& Al-Hawamdeh, S. (2001). Knowledge management in the public sector: principles and practices in police work. Journal of Information Science, 27(5), 311-318.

Luna-Reyes, R., Cresswell, A.M. \& Richardson, G. (2004). Knowledge and the development of interpersonal trust: A dynamic model. Proceedings of the 37th Hawaii International Conference on System Sciences, (HICSS'04), 3, 30086a.

Luna-Reyes, R., Black, L.J., Cresswell A.M., Pardo, T.A. (2008). Knowledge sharing and trust in collaborative requirements analysis. Systems Dynamics Review. 24(3), 269 - 297. 
Luna-Reyes, R., \& Garcia, G. (2008). E-Government and Inter-Organizational Collaboration in Mexico: Survey Results. Retrieved from: http://www.umass.edu/digitalcenter/research/working_papers/08_003LReyesEgovMexi co.pdf.

Martiny, M. (1998). Knowledge management at HP consulting. Organizational Dynamics, 27(2), 71-77.

Mathwick, C., Malhotra, N.K. and Rigdon, E. (2001). Experiential value: conceptualization, measurement and application in the catalog and Internet shopping environment. Journal of Retailing, 77(1), 39-56.

Matusik, S.F. \& Hill, SWI. (1998). The utilisation of contingent work, knowledge creation and competitive advantage. Academy of Management Review, 23, 680-697.

Maxwell, J. A. (1992). Understanding and validity in qualitative research. Harvard Educational Review, 62(3), 279-300.

McAdam, R., \& McCreedy, S. (1999). The Process of Knowledge Management within Organizations: a Critical Assessment of both Theory and Practice. Knowledge and Process Management, 6(2), 101-113.

McDermott, R., \& O'Dell, C. (2001). Overcoming cultural barriers to sharing knowledge. Journal of Knowledge Management, 5(1), 76-85.

McKenzie, N., \& Knipe, S. (2006). Research dilemmas: Paradigms, methods and methodology. Issues In Educational Research, $16 . \quad$ Retrieved from: http://www.iier.org.au/iier16/mackenzie.html

McMahon, Paul E. (2001). Virtual Project Management: Software Solutions for Today and the Future. St. Lucie Press.

McPherson, M., Smith-Lovin, L., \& Cook, James, M. (2001). Birds of a Feather: Homophily in Social Networks. Annual Review of Sociology. 27, 415-444.

Mead, S. (2001). Using social network analysis to visualize project teams. Project Management Journal, 32(4), 32-38.

Mehra, A., Smith, B.R., Dixon, A.L., \& Robertson, B. (2006). Distributed leadership in teams: The network of leadership perceptions and team performance. The Leadership Quarterly, 17, $232-245$.

Merriam, S. B. (1998). Qualitative research and case study applications in education. San Francisco, CA: Jossey-Bass.

Mertens, D.M. (2005). Research methods in education and psychology: Integrating diversity with quantitative and qualitative approaches. Thousand Oaks, CA: Sage Publications.

Meyer, J. P., \& Allen, N. J. (1997). Commitment in the workplace: Theory, research, and application. Thousand Oaks, CA: Sage Publications.

Miles, M. B., \& Huberman, A. M. (1994). Qualitative data analysis: An expandedsourcebook of new methods (2nd ed.). Thousand Oaks, CA: Sage.

Miller, L. (2007). First agencies sign up for GSN. Retrieved from: http://www.e.govt.nz/resources/news/2006/2006100501.html

Milner, E. (2000). Managing information and knowledge in the public sector. London, UK: Routledge.

Mingers, J. (2001). Combining is research methods: Towards a pluralist methodology. Information Systems Research, 12, 240-259. 
Mingers, J., \& Brocklesby, J. (1997). Multimethodology: Toward a framework for mixing methodologies. International Journal of Management Science, 25(5), 485-509.

Mintzberg, H. (1978). Patterns is strategy formation. Management Science, 24(9), 934-94.

Miranda, Shaila M., \& Saunders, Carol S. (2003). The Social Construction of Meaning: An Alternative Perspective on Information Sharing. Information Systems Research, 14(1), 87-106.

Molm, L., Takahashi, N. \& Peterson, G. (2000). Risk and trust in social exchange: an experimental test of a classical proposition. American Journal of Sociology 105(5), $1396-1427$.

Mu, J., Peng, G., \& Love, E. (2008) Interfirm networks, social capital, and knowledge flow. Journal of Knowledge Management. 12(4) 86-101.

Mueller, J., Renzl, B. \& Kaar, A. (2008). 'It's not my community' ? insights from social identity theory explaining community-failure. International Journal of Learning and Change, $3(1), 23-37$.

Mumford, E., Hirschheim, R,. Fitzgerald, G., \& T. Wood-Harper, T. (1985) Research Methods in Information Systems (eds.), Amsterdam, The Netherlands: North-Holland Publishing Co.

Murray, P., \& Myers, A. (1997). The facts about knowledge. Information Strategy, 2(7), 29-33.

Murray, S. R., \& Peyrefitte, J. (2007). Knowledge Type and Communication Media Choice in the Knowledge Transfer Process. Journal of Managerial Issues, 19(1), 111-133.

Myers, M. (1997). Qualitative Research in Information Systems. MIS Quarterly, 21(2), 241242.

Mulgan, G. (2005). Joined up government: past, present and future. Retrieved from: http://www.youngfoundation.org.uk/node/223.

Nahapiet, J. (1996). Managing relationships with global clients: Value creation through cross border networks. Paper presented at the $16^{\text {th }}$ Annual Conference of the Strategic Management Society. Phoenix, AZ.

Nahapiet, J., \& Ghoshal, S. (1998). Social capital, intellectual capital, and the organizational advantage. Academy of Management Review, 23(2), 242-258.

Newell, S. \& Swan, J. (2000). Trust and inter-organizational networking. Human Relations, 53, $10,1287-1328$.

Newell, S., Robertson, M., Scarbrough, H., \& Swan, J. (2002). Managing knowledge work. Basingstoke, UK: Palgrave.

Newell, S., Tansley, C., \& Huang, J. (2004). Social capital and knowledge integration in an erp project team: The importance of bridging and bonding. British Journal of Management, 15, 543-S57.

Nonaka, I. (1994). A dynamic theory of organizational knowledge creation. Organization Science, 5(1), 14-37.

Nonaka, I., \& Takeuchi, H. (1995). The knowledge creating company. New York: Oxford University Press.

Noteboom, B. (2002). Trust: Forms, foundations, functions, failures and figures. Cheltenham, Northampton: Edward Elgar Publishing.

Ocampo, R.B. (1995) Models of Public Management Reform: New Public Management (NPM). Asian Review of Public Administration, 248-255. 
O'Dell, C., \& Grayson, J., Jr. (1998). If only we knew what we know:The transfer of internal knowledge and best practice. USA: The Free Press.

O'Toole, L. J. J. (1997). Treating networks seriously: Practical and research-based agendas in public administration. Public Administration Review, 57(1), 545-552.

O'Toole, L. J. J., \& Meier, K. J. (2004). Public management in intergovernmental networks: Matching structural networks and managerial networking. Journal of Public Administration Research and Theory, 14(4), 469-295.

OECD. (1999) Government reform: of roles and functions of Government and public administration.Retrieved from: http://www.oecd.org/dataoecd/48/39/1910905.pdf

OECD (2001) Trends in Human Resource Management in the Public Sector. OECD

OECD. (2003). Measuring knowledge management in the business sector: OECD.

Orlikowski, W., \& Baroudi, J. J. (1991). Studying information technology in organizations: Research approaches and assumptions. Information Systems Research, 2, 1-28.

Packendorff J. (1995). Inquiring into the temporary organization: new directions for project management research. Scand J Manage 4, 319-333.

Pardo, T.A., Cresswell, A.M., Thompson, F., \& Zhang, J. (2006). Knowledge sharing in crossboundary information system development in the public sector. Information Technology and Management, 7(4), 293-313.

Pardo, T.A., \& Tayi, G.K. (2007). Interorganizational information integration: A key enabler for digital government. Government Information Quarterly, 24, 691 - 715.

Paré, G. and Elam, J. J. (1997). Using Case Study Research to Build Theories of IT Implementation. In A. S. Lee, J. Liebenau, and J. I. DeGross (eds.), Information Systems and Qualitative Research, (pp. 542-568). Tyne \& Wear, UK: Chapman \& Hall.

Parker, R., \& Bradley, L. (2000) Organizational culture in the public sector: Evidence from six organizations. International Journal of Public Sector Management, 13(2), 125-141.

Pask, G. (1984). Review of conversation theory and a protologic (or protolanguage) lp. Educational Communications and Technology, 32(1), 3-40.

Pauleen, D. (2003). Lessons learned crossing boundaries in an ICT-supported distributed team. Journal of Global Information Management, 11(4), 1-19.

Pauleen, David J. (2004). An Inductively Derived Model of Leader-Initiated Relationship Building with Virtual Team. Journal of Management nformation Systems. 20(3), 227 256.

Pearce, J. A., \& David, F. R. (1983). A social network approach to organizational designperformance. Academy of Management Review, 8(3), 436-444.

Petter, S. C., \& Gallivan, M. J. (2004). Toward a framework for classifying and guiding mixed method research in information systems. Proceedings of the 37th Hawaii International Conference on System Sciences, (HICSS'04), 8, 80257.1.

Polanyi, M. (1966). The tacit dimension. London: Routledge \& Kegan Paul.

Putnam, R. D. (1995). Bowling Alone: America's Declining Social Capital, Journal of Democracy 6(1), 65-78.

Quigley, N.R., Tesluk, P.E., Locke, E.A., \& Bartol, K.M. (2007). A multilevel investigation of the motivational mechanisms underlying knowledge sharing and performance. Organization Science, 18(1), 71-88. 
Ragin, C. C. (1994). Constructing social research: The unity and diversity of method. Thousand Oaks, CA: Pine Forge Press.

Ratnasingham, P., (1998), Internet-based EDI trust and security, Information Management \& Computer Security, 6(1), 33-39.

Remenyi, D., Williams, B., Money, A., \& Swartz, E. (1998) Doing Research in Business and Management: An Introduction to Process and Method. London, Sage Publications.

Renzl, B. (2008). Trust in management and knowledge sharing: The mediating effects of fear and knowledge documentation. Omega, 36(2), 206-220.

Rikowski, R (2007). Knowledge Management: Social, Cultural and Theoretical Perspectives. Chandos Publishing.

Ring, Peter S., \& Van de Ven, A.H. (1994) Developmental Processes of Cooperative Interorganizational Relationships. Academy of Management Review 19(1), 90-118.

Robbins, S. P., \& Decenzo, D. A. (2001). Fundamentals of management: Essential concepts and applications. NJ: Prentice-Hall.

Rooney, D. \& McKenna, B. (2005). Should the knowledge-based economy be a savant or a sage? Wisdom and socially intelligent innovation. Prometheus, 23(3), 307-323.

Ruggles, R. (1998). The state of the notion: Knowledge management in practice. California Management Review, 40(3), 80 - 89.

Ruggles, R. \& Holtshouse, D. (1999). The Knowledge Advantage. Dover, NH: Capstone.

Rush, D. (2001). Measuring connectivity at aventis pharmaceuticals. KM Review, 5(2), 10-13.

Ryan, Anne B. (2006) Post-Positivist Approaches to Research. In Researching and Writing your thesis: a guide for postgraduate students. MACE: Maynooth Adult and Community Education, pp. 12-26.

Saint-Onge, H. (1996). Tacit knowledge: The key to the strategic alignment of intellectual capital. Strategy \& Leadership. 24(2), 10-14.

Sako, M. (1992). Prices, quality and trust: Inter-firm relations in Britain and Japan. Cambridge University Press, Cambridge.

Sawyer, S. (2001). Analysis by long walk: Some approaches to the synthesis of multiple sources of evidence. In E. Trauth (Ed.), Qualitative research in IS: Issues and trends. Hershey, PA: Idea Group Publishing.

Scarbrough, H. \& Carter, C. (2000). Investigating knowledge management. London, UK: CIPD.

Scott, J. (1991). Social network analysis: A handbook. London, UK: Sage.

Seibert, S.E., Kraimer, M.L., \& Liden, R. C. (2001). A social capital theory of career success. Academy of Management Journal, 44(2), 219- 237.

Smith, H., \& McKeen, J. (2003). Instilling a knowledge sharing culture. Queen's KBE Centre for Knowledge-based Enterprise. Retrieved from: http://business.queensu.ca/kbe/docs/Smith-McKeen\%2003-11.pdf.

Snowden, D. (1999). A framework for creating a sustainable knowledge management programme. In Cortada, James. W, Woods, John A. (1999) The Knowledge Management Yearbook 1999-2000. Woburn, MA: Butterworth-Heinemann.

Spender, J. C. (1996). Making knowledge the basis of a dynamic theory of the firm. Strategic Management Journal, 17, 45-62. 
Sproull, L., \& Kiesler, S. (1991). Connections: New ways of working in the networked organization. Cambridge, MA: MIT Press.

Stenmark, D. (2001). Leveraging Tacit Organizational Knowledge. Journal of Management Information Systems, 17(3), 9-24.

Stake, R. E. (1995). The Art of Case Study Research. Thousand Oaks, CA: Sage Publications.

State Services Commission (2001). Report of the Advisory Group on the Review of the Centre. (Retrieved from http://www.ssc.govt.nz/display/document.asp?/ DocID=2776).

State Services Commission and Ministry of Social Development (2003). Review of the Centre Integrated Service Delivery: Regional Co-ordination: Final Workstream Report. Retrieved from http://www.ssc.govt.nz/ display/document.asp?DocID=4897. Last accessed 21 December, 2009).

State Services Commission (2003). The Pathfinder Project. Retrieved from: http://io.ssc.govt.nz/pathfinder/symposium.asp.

State Services Commission (2006). State of the Development Goals Report 2006. Retrieved from: http://www.ssc.govt.nz/display/document.asp?docid=5432.

State Services Commission (2007). Discussion paper: Refresh the Development Goals Milestones project. Retrieved from URL: http://www.ssc.govt.nz/discussion-paperrefresh-goals.

State Services Commission (2008). Factors for Successful Coordination - A Framework to Help State Agencies Coordinate Effectively. Retrieved from: http://www.ssc.govt.nz/display/document.asp?docid=6453.

State Services Commission (2009). Human Resource Capability Survey of Public Service Departments as at 30 June 2009 (workforce profile). Retrieved from: http://www.ssc.govt.nz/display/document.asp?docid=7493.

Su, C., Huang, M., \& Contractor, N. (2010). Understanding the structures, antecedents, and outcomes of organizational learning and knowledge transfer: A multi-theoretical and multilevel network analysis. European Journal of International Management, 4(6), 576-601.

Sutton, D. (2001). What is knowledge and can it be managed? European Journal Information Systems, 10, 80-88.

Sveiby, K-E., \& Simons, R. (2002). Collaborative Climate and Effectiveness of Knowledge Work - an Empirical Study. Journal of Knowledge Management, 6(5), 420-433.

Swan, J., Newell, S., Scarbrough, H., and Hislop, D (1999) Knowledge Management and Innovation: Networks and Networking. Journal of Knowledge Management, 3, 262275.

Swan, J., \& Newell, S. (2000). Linking knowledge management and innovation. Proceedings of the Eighth European Conference on Information Systems.

Syed-Ikshan, S., \& Rowland, F. (2004). Knowledge management in a public organisation: a study on the relationship between organizational elements and the performance of knowledge transfer. Journal of Knowledge Management, 8(2), 95-111.

Szulanski, G. (1996). Exploring internal stickiness: Impediments to the transfer of best practices within the firm. Strategic Management Journal, 17, 27-43. 
Tajfel, H. (1982). Social psychology of intergroup relations. Annual Review of Psychology, 33, 1-39 Tajfel, H. (1982). Social psychology of intergroup relations. Annual Review of Psychology, 33, 1-39.

Tajfel, H. \& Turner, J. C. (1986). The social identity theory of inter-group behavior. In S. Worchel \& L. W. Austin (Eds.), Psychology of Intergroup Relations. Chigago: Nelson-Hall.

Takeuchi, H. (1998). Beyond Knowledge Management: Lessons from Japan. Retrieved from: http://www.sveiby.com/Portals/0/articles/LessonsJapan.htm

Tang, L. (2008). Informal Interorganisational Knowledge Sharing: The Case of the Biotechnology Industry. Paper presented at the annual meeting of the NCA 94th Annual Convention, TBA, San Diego, CA. Retrieved from: //www.allacademic.com/meta/p255092_index.html.

Tashakkori, A., \& Teddlie, C. (1998). Mixed methodology: Combining qualitative and quantitative approaches. Thousand Oaks, CA: Sage.

Taylor, W. A., \& Wright, G. H. (2004). Organizational readiness for successful knowledge sharing: Challenges for public sector managers. Information Resources Management Journal, 17(2), 22-37.

Taylor, P., Richardson, J., Yeo, A., Marsh, I., Trobe, K., and Pilkington, A. (1995). Sociology in Focus. Ormskirk: Causeway Press.

Tellis, Winston, (1997). Introduction to Case Study. The Qualitative Report, 3(2). (http://www.nova.edu/ssss/QR/QR3-2/tellis1.html).

Tichy, N. M., Tushman, M. L., \& Fomburn, C. (1979). Social network analysis for organizations. Academy of Management Review, 4(4), 507-519.

Trauth, E., (2001), Qualitative Research in IS: Issues and Trends. UK/USA: Idea Group Publishing.

Tsai, W. (2002). Social structure of "coopetition" within a multiunit organization: Coordination, Competition and Interorganizational Knowledge sharing. Organization Science, 13(2), 179-190.

Tsai, W., \& Ghoshal, S. (1998). Social capital and value creation: The role of intrafirm networks. The Academy of Management Journal 41(4), 464-476.

Tuomi, I. (1999). Data is more than knowledge: implications of the reversed knowledge hierarchy for knowledge management and organizational memory. Journal of Management Information Systems, 16(3), 107-121.

Van den Hooff, B. \& Huysman, M. (2009). Managing knowledge sharing: Emergent and engineering approaches. Information \& Management 46(1), 1-8.

Van den Hooff, B., \& Ridder, J.A. (2004) Knowledge sharing in context: The influence of organizational commitment, communication climate and CMC use on knowledge sharing. Journal of Knowledge Management. 8(6), 117-130.

Van den Hooff, B. \& Schipper, S. (2009). Competitive Organizational Cultures and Knowledge Sharing. Paper presented at the annual meeting of the International Communication Association, Sheraton, New York. Retrieved from: http://www.allacademic.com/meta/p12940_index.html.

Van Wijk, R., Jansen, J.J.P. and Lyles, M.A. (2008). Inter- and intra-organizational knowledge transfer: A meta-analytic review and assessment of its antecendents and consequences. Journal of Management Studies, 45, 830-853. 
Venters,W. (2001) Review of the literature on Knowledge Management . C-SanD Working Paper. Retrieved from: http://www.c-sand.org.uk/Documents/WP1001-02KMLitRev.pdf

Wah, C. Y., Menkhoff, T., Loh, B., \& Evers. H.D. (2007). Social Capital and Knowledge Sharing in Knowledge-Based Organizations: An Empirical Study. International Journal of Knowledge Management, 3(1), pg 29-48.

Walker, A. (2004) Overcoming the Neoliberal Legacy: The Importance of Trust for Improved Interagency Collaborative Working in New Zealand, Research Paper Number 11, School of Government, Victoria University of Wellington, Wellington

Walsham, G. (1995). Interpretive case studies in is research: Nature and method. European Journal of Information Systems, 4, 74-81.

Wasko, M. \& Faraj, S. (2005). Why should I share? Examining social capital and knowledge contribution in electronic networks of practice. MIS Quarterly, 29(1), 35-57.

Wasserman, S., \& Faust, K. (1994). Social network analysis: Methods and applications. Cambridge, UK: Cambridge University Press.

Webster, J. \& Trevino, Klebe, L. (1995). Rational and social theories as complementary explanations of communication media choices: Two policy-capturing studies. Academy of Management Journal, 38(6), 1544-1572.

Weick, K. (1995). Sensemaking in organizations. Thousand Oaks: California: Sage.

Wikipedia (2010). Retrieved from: http://en.wikipedia.org/wiki/Public_sector

Widen-Wulff, G. \& Ginman, M. (2004). Explaining knowledge sharing in organizations through the dimensions of social capital. Journal of Information Science, 30(5), 448-458.

Wikström, S., Normann, R. Anell, B., Ekvall, G., Forslin, J. and Stearad, P.H. (1994), Knowledge and Value. The Company as a Knowledge Processing and Value Creating System. London, UK: Routledge

Wiig, K. M. (2002). Knowledge management in public administration. Journal of Knowledge Management, 6(3), 224 - 239.

Willem, A. (2002). Leveraging knowledge integration through social identity: A knowledgebased theory of the firm perspective. Academy of Management Annual Conference, Denver, Colorado, 12-14 August 2002.

Willem, A. \& Buelens, M. (2007). Knowledge Sharing in Public Sector Organizations: The Effect of Organizational Characteristics on Interdepartmental Knowledge Sharing. Working Paper. Retrieved from: http://www.feb.ugent.be/nl/Ondz/wp/Papers/wp_05_344.pdf.

Willem A., \& Buelens, M. (2009). Knowledge sharing in inter-unit cooperative episodes. The impact of organizational structure dimensions. International Journal of Information Management, 29(2), 151 -160.

Winter, S. G. (1995). Four Rs of profitability: Rents, resources, routines and replication. In Montgomery, C. A. (ed.), Resource-Based and Evolutionary Theories of the Firm. Norwell, MA: Kluwer.

Woolcock, M., (2001). The Place of Social Capital in Understanding Social and Economic Outcomes. Isuma 2(1), 11-17.

WorldBank. (2005). Social capital and the public sector. Retrieved from http://web.worldbank.org/WBSITE/EXTERNAL/TOPICS/EXTSOCIALDEVELOPME 
NT/EXTTSOCIALCAPITAL/0,contentMDK:20185275 menuPK:404103 pagePK:148 956 piPK:216618 theSitePK:401015,00.html.

Wright, Bradley, E. (2003). Toward Understanding Task, Mission and Public Service Motivation: A Conceptual and Empirical Synthesis of Goal Theory and Public Service Motivation Paper prepared for presentation at the 7th National Public Management Research Conference, Georgetown Public Policy Institute, Georgetown University, Washington, D.C.

Wu, Wei-Li, Hsu, Bi-Fen \& Yeh, Ryh-Song Yeh (2007). Fostering the determinants of knowledge transfer: a team-level analysis. Journal of Information Science. 33, 326339.

Wu, Wei-Li; Lin, Chien-Hsin; Hsu, Bi-Fen; Yeh, Ryh-Song (2009) Interpersonal trust and knowledge sharing: Moderating effects of individual altruism and a social interaction environment. Social Behavior and Personality: An International Journal, 37(1), pp. 8393.

Yang, J-T. (2006). Knowledge sharing: Investigating appropriate leadership roles and collaborative culture. Tourism Management, 28(2), 530-543.

Yin, R. K. (1989). Case study research design and methods. Newbury Park, CA: Sage.

Yin, R. K. (1993). Applications of case study research. Newbury Park, CA: Sage.

Yin, R. K. (1994). Case study research, design and methods (2nd ed.). Newbury Park, CA: Sage.

Yin, R. K. (2004). Case study research, design and methods (3rd ed.) Newbury Park, CA: Sage.

Zack, Michael, H. (2000). Researching Organizational Systems using Social Network Analysis. Proceedings of the 33rd Hawaii International Conference on Systems Sciences, Maui, Hawaii.

Zenun, M. M. N., Loureiro, G., \& Araujo C. S. (2007). The Effects of Teams' Co-location on Project Performance. In Loureiro, G., \& Curran, R. (Eds.,) Complex Systems Concurrent Engineering-Collaboration, Technology Innovation and Sustainability (pp. 717-726) London, UK: Springer.

Zucker L.G. (1986) Production of Trust: Institutional Sources of Economic Structure, 18401920 in Research in Organizational Behavior, 8, 53-111 


\title{
APPENDIX
}

\section{Appendix 1: Initial research enquiry letter sent to public sector organisations}

[Participant Name]

[Title]

[Organisation]

[Address]

[City]

\author{
VICTORIA UNIVERSITY OF WELLINGTON \\ Te Whare Wänanga o te Opoko o te Ika a Mäsi
}

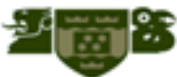

10 January 2006

Dear [name]

\section{Doctoral Thesis:}

Knowledge Sharing in Inter-agency Projects in the Public Sector:

The role of Information and Communication Technologies (ICT) and Social Capital

I am currently completing a doctoral thes is in the School of Information Management at Victoria University.

This study is supported by a Top Achiever Doctoral Scholarship awarded by the Tertiary Education

Commis sion in 2004. The study explores knowledge sharing behaviours within interagency projects in the New Zealand public sector, and will be carried out in three phases. You are invited to participate in Phase One of the study in which data will be collected from key project staff through face-to-face interviews.

The study will identify how knowledge is shared within collaborative projects. More specifically, the study will explore the influence of ICT, and social capital (how informal relationships are formed), in the development of knowledge sharing networks within the projects. The outcome of the study will be the development of a knowledge sharing framework specific to the unique factors of the public sector. A full outline of the research is contained in the attached information sheet.

Ethical consent for this study has been approved by the University Informatics Human Ethics committee. All data collected will remain confidential to the investigator and the supervisor, and organisations, participants and their opinions will not be identifiable.

The supervisors for this study are Dr David Pauleen and Profess or Sid Huff. If you wish to discuss this study with either myself or my supervisors, we may be contacted on:
Sally Dexter
$\mathrm{Ph}:(04) 4721000$
Doctoral Student
Email: sally.dexter@vuw.ac.nz
Dr David Pauleen
Senior Lecturer
$\mathrm{Ph}:(04)$ 463-6886
Email: david.pauleen@vuw.ac.nz
Professor Sid Huff
Head of School
$\mathrm{Ph}$ : (04) 463-5819
Email: sid.huff@vuw.ac.nz

Thank you foryour participation in this project, I will call you in the next few days to arrange a convenient interview time.

Yours sincerely

Sally Dexter

$\mathrm{PhD}$ student 


\section{Appendix 2: Participant Information Sheet}

\section{Participant information sheet}

Dear $<$ Participant Name>

Re: A Study of Knowledge sharing in inter-agency projects in the Public Sector: The role of ICT and social capital

Thank you for participating in this doctoral research study. This sheet outlines key information relating to the collection of data through interviews with key project staff, as well a protocol sheet outlining some basic questions that will be asked.

Researcher:

Sally Dexter: School of Information Management Victoria University of Wellington

Purpose of the study:

I am a doctoral student in the School of Information Management at VictoriaUniversity of Wellington. As part of this degree I am undertaking a res earch project leading to a thesis. The project I am undertaking explores knowledge sharing behaviours within collaborative, inter-agency projects in the New Zealand public sector. Specifically, the study will examine:

How knowledge is shared across teame in multi-agency collaborative projects within the New Zealand public sector?

How ICT influences the development of the knowledges haring network?

How social capital influences the development of the knowledge shaving network?

How social capital and ICT influence each other?

Although the research addresses the influence of ICT, the projects do not need to be technology projects. Rather the focus will be on how technology is used by group members for the purpo se of knowledge sharing and could, for example, involve services including email, internet, or groupware facilities.

The thesis will be submitted for marking to the School of Information Management and deposited in the University Library. It is intended that one or more articles will be submitted for publication in scholarly journals.

\section{Ethical Approval:}

The University requires that ethics approval be obtained for research involving human participants. This study has been granted ethical approval and will be conducted according to the University's strict ethical regulations and written consent sought from each participant. In keeping with this policy, all data collected would remain confidential and would be destroyed after two years of submission and evaluation of the thesis.

\section{Data Collection:}

Res earch data will be collected in three phases using a combination of interviews and questionnaires, as outlined below.

\section{Phase One:}

- Face-to-face interviews with key project staff, and

Phase Two:

- Completion of a questionnaire by all project staff.

Phase Three:

- Face-to-face interviews with key project staff

You are invited to participate in Phase $\mathrm{Qne}$ of the study, in which data will be collected via face-to-face interviews. (dhis was amended to reflect the particular stage in which the individual was invited to participats) 
Each phase of the research will be linked to a specific project milestone or event, however specific timescales are likely to vary in duration and data collection will be adjusted to fit with the precise nature of each project. Participants may withdraw at any point up to before commencement of Phase One.

\section{Data Storage:}

Written information will be securely stored in a locked cabinet with access restricted to the investigator. Electronic information will be password protected and access restricted to the investigator. All data will be destroyed two years following the submission of the thesis.

\section{Contact Details:}

Investigator:

Sally Dexter $\quad \mathrm{Ph}:(04) 4721000$ ext 8993

Email: sally.dexter@vuw.ac.nz

Supervisors:

Dr David Pauleen

Senior Lecturer

Ph: (04) 463-6886

Email: david.pauleen@vuw.ac.nz

Profess or Sid Huff

Head of School

$\mathrm{Ph}:(04) 463-5819$

Email: sid.huff@vw.acnz

Please feel free to contact either mys elf or my supervisors with any questions you may have. Once again, thank you for your participation in this study.

Yours sincerely

Sally Dexter

$\mathrm{PhD}$ student, School of Information Management 
Interview protocol and ex ample questions

\section{Research Study: \\ Knowledge sharing in inter-agency projects in the Public Sector:} The role of ICT and social capital

Interview questions will seek to explore the research objectives, as well as investigate any areas of interest that arise during the interview. Interviews will be conducted in a semi-structured format to include a number of questions that have been prepared in advance (see below). These questions will be used as a guide throughout the interview process, while allowing flexibility to follow lines of questioning that occur up during the interview process.

Examples of research questions include:

1. What is the purpose of this project?

2. What is the official name of the project?

3. How have team members been selected?

4. What is the nature of teammember's engagement?

5. To what extent to team members know each other before theyjoin the project?

6. What specific activities occur to help teammembers get to know each other?

7. Is knowledge sharing discussed as an activity of the project?

8. How does knowledge sharing occur within the team? (i,e. face-to-face, by email etc).

9. What do you see as the knowledge sharing challenges faced by inter-organisational teams.

10. How do you think these challenges may differ between the public and private sectors?

11. How important do you think information and communication technologies are in enabling individuals to share information and knowledge?

12. What other factors do you think think influences people's willingness to shareknowledge? 


\section{VICTORIA UNIVERSITY OF WELLINGTON}

Te Whare Wänanga o te Opoko o te Ika a Mäni

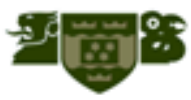

\section{Consent to participation in research}

Title of project:

Knowledge sharing in inter-agency projects in the Public Sector: The role of ICT and social capital

I have been given and have understood an explanation of this research project. I have had an opportunity to ask questions and have them an swered to my satis faction. I understand that I may withdraw from this project at any point up to February $15^{\mathrm{m}}, 2007$

I understand that any information I provide will be kept confidential to the researcher and the supervis or, the published results will not use my name, and that no opinions will be attributed to me in any way that will identify me. I consent to the interview being tape recorded and understand that the tape will be transcribed by the interviewer and kept confidential to the interviewer, and that I will have an opportunity to check the transcripts of the interview before publication. I understand that the tape recording of interviews will be stored for a period of two years following the submission and evaluation of the thesis.

I agree to take part in this research.

Signed:

Date: 


\section{Appendix 3: Contact Summary Sheet}

\begin{tabular}{|l|l|}
\multicolumn{2}{c|}{ Contact Summary Form } \\
\hline Name & $x 0000 x$ \\
\hline Site & $x 000 x$ \\
\hline Contact Date & $x 000 x$ \\
\hline Summary Date & $x 000 x$ \\
\hline
\end{tabular}

Main Points of Interest:

- Able to view project team members from meeting room. Lots of interaction and laughter.

- Very important project to interviewee [team leader], stressed the importance of the project numerous times. Some responses indicate that the project may not be valued as highly by the organisation as it is by the project team leader.

- Highly informal, despite organisation being formal in approach.

- Lack of ICT focus

- Aware of SC aspects, but no specific action in that regard.

Summary of Target Question Information:

\begin{tabular}{|l|ll|}
\hline Project Overview & - Share data across organisations. \\
& - & No formal process - this team follows general rules of working. \\
& - & Nommunication/decision-making centralised. \\
& & core team. \\
\hline Knowledge & - & No differentiation between information and knowledge. \\
& - & Project focus, mainly data/information. \\
& - & No formal thought given to knowledge sharing, but important to interviewee. \\
& - & Information sharing seen as risky - often overseen by higher management. \\
\hline ICT aspects & - & Interviewee not particularly ICT savvy - seems unsure talking about technology. \\
& - & No specific focus on ICT to enable sharing - transfer done manually via CD. \\
& - & Different applications for analysis. \\
\hline SC aspects & - & Lack of trust between organisations. \\
& - & Lack of trust within organisation. \\
& - & Lack of relationship building with individuals from other grganisation. \\
& - & Organisational team described as tight-knit group. \\
& - & Thinks more focus on social/relational aspects needed, but not sure how to instigate. \\
\hline Challenges & - Building trust - this is the main issue. \\
& - Understanding what each other mean - using different language. \\
& - Time.
\end{tabular}

New questions/themes arising from contact:

- What specific action does the team leader take to help build relationships across the organisations?

- How does centralised decision-making (by higher management) impact on the day-to-day operations of the project team?

- How does centralised decision-making affect individual's commitment to the project and sense of empowerment?

- What has been learnt from the annual repetition of the project?

Additional points of note:

- Talked a lot about own role. Got impression feels undervalued and that many decisions are taken by higher management in regard to knowledge sharing. 


\section{Appendix 4: Document Summary Form}

\section{Document Summary Form}

\begin{tabular}{|l|l|}
\hline Document & Powerooint slides outlining project purpose \\
\hline Case/Org & xxxxx \\
\hline Contact & \\
\hline Date of receipt & xxxxx \\
\hline
\end{tabular}

Description of Document:

- 12 powerooint slide (print-out) outlining the main points of the project. Was used to communicate objectives to wider stakeholders.

Summary of Contents:

- Project Name

- Key Objectives of project

- Timeframe and milestones

- Impact on wider stakeholders

- How it fits into the larger picture

\section{Significance/Importance of Document:}

- Provides formal overview of project and positions it in the overall programme of work.

- Provides high level of project team

- Provides context for project activities. 


\title{
Appendix 5: Survey
}

\author{
VICTORIA UNIVERSITY OF WELLINGTON \\ Te Whare Wānanga o te Ūpoko o te Ika a Māui

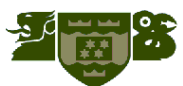 \\ KNOWLEDGE SHARING IN INTERAGENCY PROJECTS \\ IN THE NEW ZEALAND PUBLIC SECTOR
}

\begin{abstract}
Dear Participant
Thank you for participating in my doctoral research by completing this questionnaire. Your participation is much appreciated, and is essential to the quality of data collected. The questionnaire forms part of a study to develop understanding of public sector based collaboration. The research explores how information and knowledge sharing networks develop within inter-agency projects, and the factors that influence that development. The study has been approved by the Informatics Ethics Committee of Victoria University of Wellington.

This questionnaire should take no more than 15 minutes. By completing and returning the questionnaire you acknowledge your consent to participation in this study. Your responses, as well as your personal identity will remain completely confidential to the researcher. Written information will be securely stored in a locked cabinet with access restricted to the investigator. Electronic information will be password protected and access resiricted to the investigator. All data will be destroyed two years following the submission of the thesis. You do not need to write your name on the questionnaire, as it has been confidentially coded so that the researcher can ascertain which participants have responded. On completion of the thesis a report outlining the overall findings of the research will be sent to each participating organization. The report will not identify any individual or their responses.
\end{abstract}

The questions relate to your experience and perceptions of the collaborative work in which you are involved. This work is referred to as a project, but includes projects, programmes, and on-going collaborative work between multiple agencies/organizations. There are no right or wrong answers, and the openness of your response will increase the quality and value of the data collected.

Once complete, I would appreciate it if you could please return the questionnaire and the consent form in the freepost envelope provided as soon as possible.. Thank you for your participation.

\section{BACKGROUND INFORMATION}

\begin{tabular}{|l|l|}
\hline $\begin{array}{l}\text { Tenure in your parent organization (the organization } \\
\text { in which you are normally based) }\end{array}$ & \\
\hline The position you hold in your parent organization & \\
\hline $\begin{array}{l}\text { Have you been part of this project from its } \\
\text { beginning? }\end{array}$ & YES/NO \\
\hline $\begin{array}{l}\text { If you answered no to the above question, please } \\
\text { state the approximate date at which you joined the } \\
\text { group }\end{array}$ & \\
\hline
\end{tabular}

For more information, please do not hesitate to contact:

Sally Dexter, School of Information Management, Victoria University

Email: Sally.Dexter@vuw.ac.nz,Tel: (04) 4789161

Or the study supervisors:

Dr David Pauleen, Senior Lecturer. Email: David.Pauleen@wuw.ac.nz, Tel: (04) 4636886

Prof. Sid Huff, Head of School. Email: Sid.Huff@vuw.ac.nz, Tel: (04) 4635819

Thank you very much for your help and co-operation. 
Please answer the questions below by placing an ' $x$ ' in the corresponding column on the list of staff opposite. NOTE: If there is anyone with whom you communicate about this project but who is not named, please write their name in one of the free spaces provided and include them in the questions below.

1. Who do you go to for project related help or advice?

2. Who comes to you for project related help or advice?

3. Who do you consider a friend as well as a work colleague?

4. Which group members do you meet with on a social basis?

For ONLY those people who have selected in questions 1 and 2 , please complete the following questions by placing your answer in the corresponding column.

5. What is this person's physical proximity to you?
Same floor of same building
b. Different floor of same building
c. Different building
d. Different city

6. How did you first meet this person?
. On this project team
b. Worked together previously
c. Have not worked together, but are part of the same business related networks
d. Non-work related connections

7. In general, how often do you communicate with this person?
a. Once a day
b. More than once a day
c. Once a week
d. More than once a week
e. Less than once a week

8. How do you most typically communicate with this person?

(You may select more than one option)
a. Face-to-face meeting
b. Telephone
c. Email
d. Other, please specify

9. What motivates you most to share your knowledge with this person?
a. Trust in the individual
b. The feeling that I am able to help that person
c. The belief that the recipient will share their knowledge with me in return
d. Commitment to the project
e. Presence of technology that enables easy knowledge sharing
f. Recognition of my knowledge and expertise
g. Other, please specify

10. Which communication channels do you most often use to share knowledge?

a. Face-to-face meetings

b. Telephone

c. Email

d. Intranet/web based technology

e. Other, please specify 


\begin{tabular}{|l|l|l|l|l|l|l|l|l|l|l|}
\hline Name: & Q1 & Q2 & Q3 & Q4 & Q5 & Q6 & Q7 & Q8 & Q9 & Q10 \\
\hline [participant name] & & & & & & & & & & \\
\hline [participant name] & & & & & & & & & & \\
\hline [participant name] & & & & & & & & & & \\
\hline [participant name] & & & & & & & & & & \\
\hline [participant name] & & & & & & & & & & \\
\hline [participant name] & & & & & & & & & & \\
\hline [participant name] & & & & & & & & & & \\
\hline [participant name] & & & & & & & & & & \\
\hline [participant name] & & & & & & & & & & \\
\hline [participant name] & & & & & & & & & & \\
\hline [participant name] & & & & & & & & & & \\
\hline [participant name] & & & & & & & & & & \\
\hline [participant name] & & & & & & & & & & \\
\hline
\end{tabular}




\begin{tabular}{|c|c|c|c|c|c|c|c|c|}
\hline & HIS PROJECT: & $\begin{array}{l}\text { Strongly } \\
\text { Disagre }\end{array}$ & ly 1 .... & $\ldots . . .2$ & & $\ldots \ldots . . . .4$. & $\ldots \ldots . .5$ & $\begin{array}{l}\text { Strongly } \\
\text { Agree }\end{array}$ \\
\hline 12 & $\begin{array}{l}\text { There is a formal hierarchy (ie people in this group are at } \\
\text { different levels of seniority) }\end{array}$ & SD & 0 & 0 & 0 & 0 & 0 & SA \\
\hline 13 & $\begin{array}{l}\text { There are formal processes and procedures, known by all } \\
\text { team members, that guide how work will be carried out }\end{array}$ & SD & 0 & 0 & 0 & 0 & 0 & SA \\
\hline 14 & $\begin{array}{l}\text { Pressures on this project sometimes necessitate that formal } \\
\text { processes and procedures are not followed }\end{array}$ & SD & 0 & 0 & 0 & 0 & 0 & SA \\
\hline 15 & $\begin{array}{l}\text { I can approach another group member for help without } \\
\text { going through a formal procedure or the chain of command }\end{array}$ & SD & 0 & 0 & 0 & 0 & 0 & SA \\
\hline 16 & $\begin{array}{l}\text { Decision making is allocated across the group, so decisions } \\
\text { are not made by one individual }\end{array}$ & SD & 0 & 0 & 0 & 0 & 0 & SA \\
\hline 17 & $\begin{array}{l}\text { Within this group, I know which decisions I am allowed to } \\
\text { make and which I need to consult others on }\end{array}$ & SD & 0 & 0 & 0 & 0 & 0 & SA \\
\hline 18 & $\begin{array}{l}\text { Tasks are assigned to the individual with the most relevant } \\
\text { expertise }\end{array}$ & SD & 0 & 0 & 0 & 0 & 0 & SA \\
\hline 19 & $\begin{array}{l}\text { I have enough time to consult with other group members } \\
\text { and to make myself available to others }\end{array}$ & SD & 0 & 0 & 0 & 0 & 0 & SA \\
\hline 20 & $\begin{array}{l}\text { Open communication with all team members is actively } \\
\text { encouraged }\end{array}$ & SD & 0 & 0 & 0 & 0 & 0 & SA \\
\hline 21 & $\begin{array}{l}\text { The physical space in which the group is located facilitates } \\
\text { spontaneous communication }\end{array}$ & SD & $\bigcirc$ & 0 & 0 & 0 & 0 & SA \\
\hline 22 & $\begin{array}{l}\text { The more familiar I am with a team member the more likely I } \\
\text { am to communicate with them face-to-face }\end{array}$ & SD & 0 & 0 & 0 & 0 & 0 & SA \\
\hline 23 & $\begin{array}{l}\text { It is difficult to develop a good working relationship with } \\
\text { groups members without face-to-face contact }\end{array}$ & SD & 0 & 0 & 0 & 0 & 0 & SA \\
\hline
\end{tabular}




\begin{tabular}{|c|c|c|c|c|c|c|c|}
\hline \multicolumn{2}{|c|}{ IN THIS PROJECT: } & $\begin{array}{l}\text { Strongly } \\
\text { Disagree }\end{array}$ & e 1 ....... & $\ldots . .2 \ldots \ldots$. & \multicolumn{2}{|c|}{$\ldots 3 \ldots \ldots \ldots \ldots \ldots \ldots$} & .5 Agree \\
\hline 24 & $\begin{array}{l}\text { The dynamics of the group are dependent on one or a few } \\
\text { individuals within the team }\end{array}$ & SD & $\bigcirc$ & 0 & $\bigcirc$ & $\bigcirc$ & SA \\
\hline 25 & $\begin{array}{l}\text { The culture of this group is different to the culture of my own } \\
\text { organisation (the organisation in which you are usually } \\
\text { based) }\end{array}$ & SD & $\bigcirc$ & $\bigcirc$ & $\bigcirc$ & $\bigcirc$ & SA \\
\hline 26 & $\begin{array}{l}\text { It is difficult for individual organisational cultures to be } \\
\text { merged within a single project team }\end{array}$ & SD & $\mathrm{O}$ & 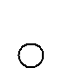 & 0 & $\bigcirc$ & SA \\
\hline 27 & $\begin{array}{l}\text { I sometimes find it difficult to balance the interests of my } \\
\text { own organisation with the interests of the group }\end{array}$ & SD & $\bigcirc$ & $\bigcirc$ & $\bigcirc$ & $\mathrm{O}$ & O SA \\
\hline 28 & Trust is an important aspect of working collaboratively & SD & 0 & 0 & 0 & 0 & O SA \\
\hline 29 & $\begin{array}{l}\text { Trust is based on how well you know a person rather than } \\
\text { how competent or effective they are }\end{array}$ & SD & $\bigcirc$ & 0 & $\mathrm{O}$ & $\mathrm{O}$ & O SA \\
\hline 30 & $\begin{array}{l}\text { Getting to know someone through a social or informal } \\
\text { function is the best way of building trust }\end{array}$ & SD & $\bigcirc$ & 0 & $\bigcirc$ & $\mathrm{O}$ & SA \\
\hline 31 & $\begin{array}{l}\text { There have been formal initiatives (ie team building or } \\
\text { organised social functions) to actively build trust among } \\
\text { group members }\end{array}$ & SD & 0 & 0 & $\mathrm{O}$ & 0 & SA \\
\hline 32 & I consider trust levels within this group to be above average & SD & 0 & 0 & 0 & 0 & SA \\
\hline 33 & $\begin{array}{l}\text { I consider lunches with group members or drinks after work } \\
\text { to be an important part of team building }\end{array}$ & SD & $\bigcirc$ & 0 & $\mathrm{O}$ & 0 & O SA \\
\hline 34 & Email communications help establish trust & SD & $\bigcirc$ & O & 0 & 0 & SA \\
\hline
\end{tabular}


Many organisations now make formal differentiation between information and knowledge. Please indicate your response by circling the number on the corresponding scale, where 1 indicates that you strongly DISAGREE with the statement and 5 indicates you strongly AGREE with the statement.

IN THIS PROJECT:

Strongly

Strongly

35 People clearly understand the difference between information and knowledge
SD

SA

36 There are formal processes and procedures that detail how information is shared on this project
SD
$\bigcirc \bigcirc \bigcirc$ SA

37 Information is promptly made available to group members rather than having to seek it from someone at a higher level

38 Individuals willingly share information

SD $\bigcirc \bigcirc \bigcirc \bigcirc$ SA

39 When I need information, I am more likely to contact someone who is likely to have that information rather than $\quad$ SD $\bigcirc \quad \bigcirc \quad 0 \quad \bigcirc \quad 0$ SA look through my own files

sto

40 Information is easier to find if it is located on a central electronic workspace/intranet/shared drive that all group

SD $\bigcirc \bigcirc \bigcirc \bigcirc$ SA members can access

41 There are formal processes and procedures that detail how knowledge is shared on this project

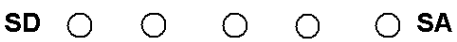

42 Individuals willingly share their knowledge
SD
$\bigcirc$
$\bigcirc$ SA

43 Group members are actively encouraged to share their knowledge

SD $\bigcirc \bigcirc \bigcirc \bigcirc$ SA

44 I am more comfortable sharing my knowledge with the members of the group I know best

SD $\bigcirc \bigcirc \quad \bigcirc \quad \bigcirc \quad$ SA

45 I expect that knowledge sharing will be reciprocal

SD $\bigcirc \bigcirc \bigcirc \bigcirc$ SA

46 Some individuals in this group share their knowledge more easily than others

SD $O \bigcirc \bigcirc \bigcirc$ SA

47 I often learn more from an informal chat than through a formal meeting

SD $\bigcirc \bigcirc \bigcirc \bigcirc$ SA 
The following questions relate to the use of technology. Technology includes, but is not exclusive to, databases, intranets, shared workspaces, email, electronic discussion boards and so on. Please indicate your response by circling the number on the corresponding scale, where 1 indicates that you strongly DISAGREE with the statement and 5 indicates you strongly AGREE with the statement.

\begin{tabular}{|c|c|c|c|c|c|c|c|c|}
\hline \multicolumn{2}{|c|}{ IN THIS PROJECT: } & $\begin{array}{l}\text { Strongly } \\
\text { Disagree }\end{array}$ & & $\ldots 2 \ldots \ldots$. & \multicolumn{3}{|c|}{$. .3 \ldots \ldots \ldots \ldots \ldots \ldots \ldots \ldots . .5$} & $\begin{array}{l}\text { Strongly } \\
\text { Agree }\end{array}$ \\
\hline 48 & There are a range of technologies are available for use & SD & $\bigcirc$ & 0 & 0 & 0 & 0 & SA \\
\hline 49 & In general, I find these technologies effective & SD & $\bigcirc$ & $\bigcirc$ & $\mathrm{O}$ & $\bigcirc$ & $O$ & SA \\
\hline 50 & $\begin{array}{l}\text { I prefer to share information and knowledge through } \\
\text { technology, rather than face-to-face }\end{array}$ & SD & 0 & 0 & 0 & 0 & 0 & SA \\
\hline 51 & $\begin{array}{l}\text { This project would benefit from a wider range of } \\
\text { technologies being available }\end{array}$ & SD & $\bigcirc$ & $\bigcirc$ & 0 & 0 & $\bigcirc$ & SA \\
\hline 52 & Technology makes it easy to share information & SD & $\bigcirc$ & $\bigcirc$ & O & $\bigcirc$ & 0 & SA \\
\hline 53 & $\begin{array}{l}\text { In my organization (the organization in which you are } \\
\text { normally based) there is a strong emphasis on using } \\
\text { technology to share information }\end{array}$ & SD & 0 & $\bigcirc$ & $\bigcirc$ & $\bigcirc$ & 0 & SA \\
\hline
\end{tabular}

Please answer the questions below in your own words:

54. I define knowledge as:

55. What are the three most important challenges that collaborative projects face?

56.

What are the key barriers to knowledge sharing in collaborative projects?

Thank you for participating in this research. 
Appendix 6: SNA data entry files
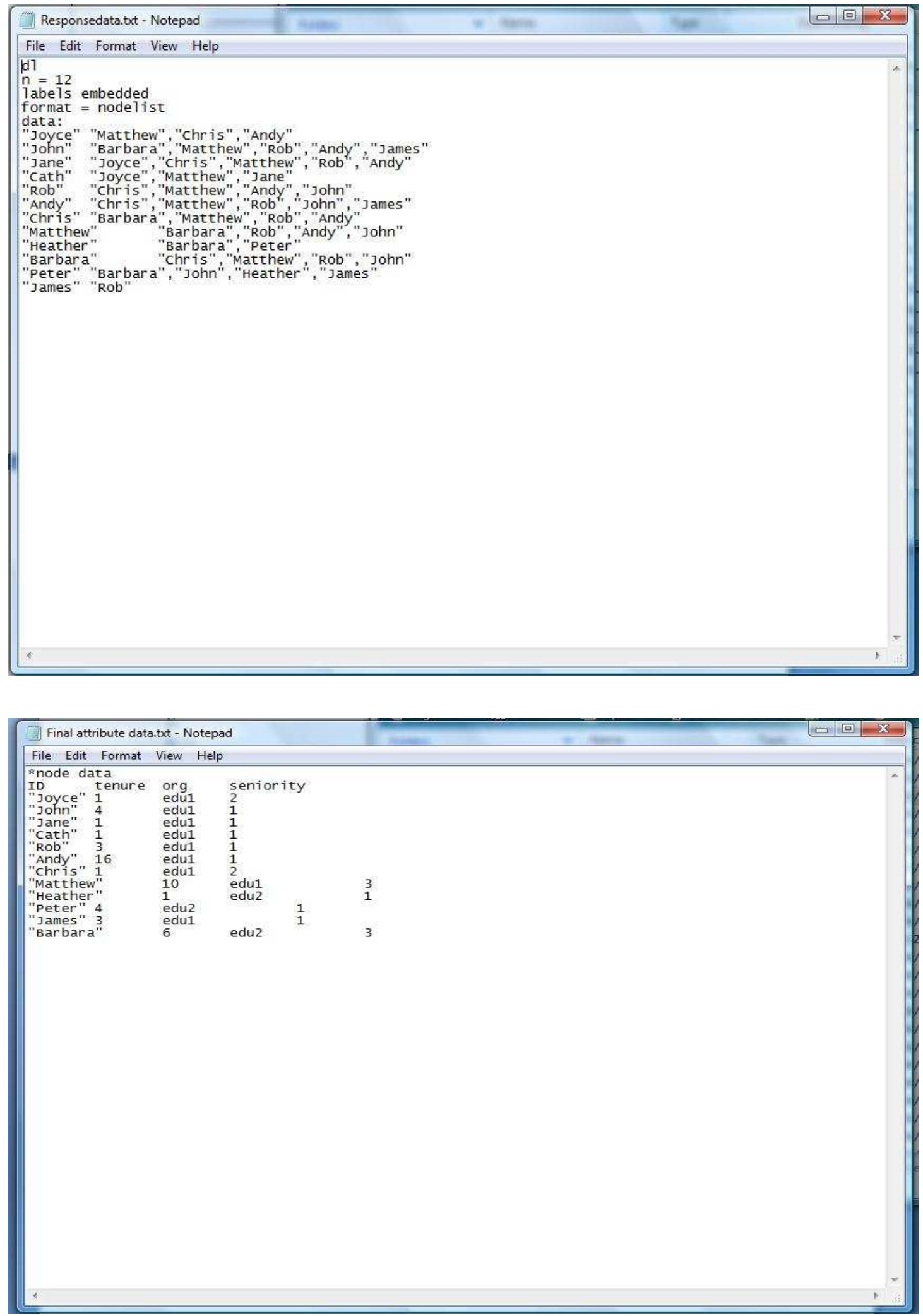
Appendix 7: Extract from research coding tree

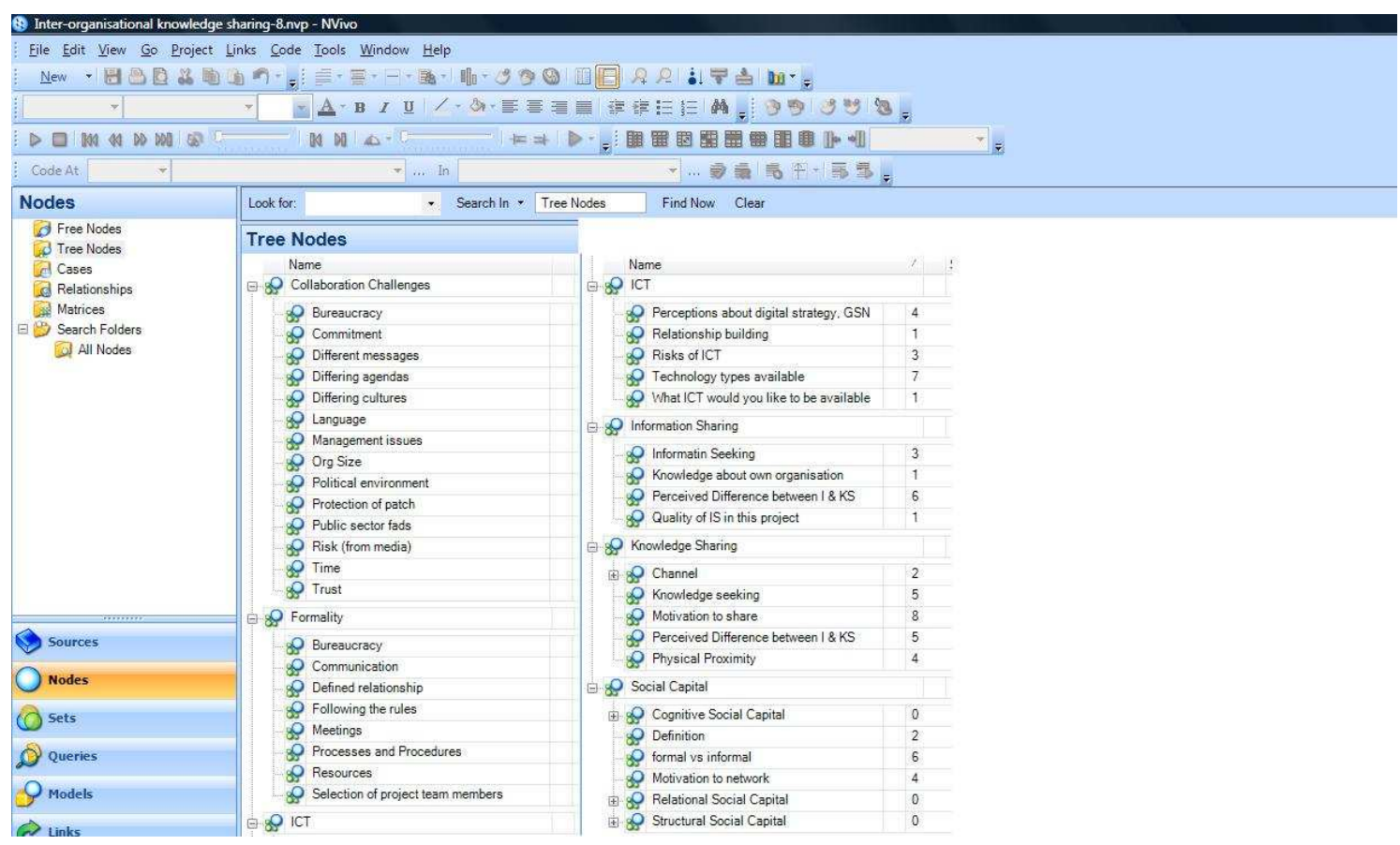


Appendix 8: Participant Details

\begin{tabular}{|c|c|c|c|c|c|c|c|}
\hline Case & Name & Role & Organisation & $\begin{array}{l}\text { Tenure in } \\
\text { Organisation } \\
\text { (years) }\end{array}$ & $\begin{array}{c}\text { Phase } \\
1 \\
\end{array}$ & $\begin{array}{c}\text { Phase } \\
2 \\
\end{array}$ & $\begin{array}{c}\text { Phase } \\
\mathbf{3}\end{array}$ \\
\hline 1 & Joyce & Senior Researcher & Edu1 & 1 & & $x$ & \\
\hline 1 & John & Business Analyst & Edu1 & 3.5 & & $\mathrm{x}$ & \\
\hline 1 & Jane & Research/Analyst & Edu1 & 0.6 & & $\mathrm{x}$ & \\
\hline 1 & Cath & Administrator & Edu1 & 0.1 & & $x$ & \\
\hline 1 & Rob & Analyst & Edu1 & 3 & & $\mathrm{x}$ & \\
\hline 1 & Andy & Not Stated & Edu1 & 16 & & $\mathrm{x}$ & $\mathrm{x}$ \\
\hline 1 & Chris & Senior Researcher & Edu1 & 0.6 & & $x$ & $\mathrm{x}$ \\
\hline 1 & James & Business Analyst & Edu1 & 3 & & $\mathrm{x}$ & \\
\hline 1 & Matthew & Manager & Edu1 & 4 & $\mathrm{x}$ & $\mathrm{x}$ & \\
\hline 1 & Barbara & Team Leader & Edu2 & 6 & $\mathrm{x}$ & $\mathrm{x}$ & \\
\hline 1 & Peter & Research Analyst & Edu2 & 1 & & $\mathrm{x}$ & \\
\hline 1 & Heather & Research Analyst & Edu2 & 0.2 & & $x$ & $x$ \\
\hline 2 & Donna & $\begin{array}{l}\text { Senior Research } \\
\text { Adviser }\end{array}$ & Jus1 & 19 & & $\mathrm{x}$ & \\
\hline 2 & Joe & Business Data Analyst & Jus 1 & 8 & & $\mathrm{x}$ & $\mathrm{x}$ \\
\hline 2 & Cliff & $\begin{array}{l}\text { Data Warehouse } \\
\text { Architect }\end{array}$ & Jus1 & 2.5 & & $x$ & \\
\hline 2 & Peter & Strategic Technologist & Jus1 & 5 & & $\mathrm{x}$ & $x$ \\
\hline 2 & Martin & Manager & Jus 1 & 8 & $\mathrm{x}$ & $\mathrm{x}$ & \\
\hline 2 & Katy & Data Modeller & Jus 4 & 1 & & $x$ & \\
\hline 2 & Zoe & Senior Business Adviser & Jus5 & 1 & & $\mathrm{x}$ & \\
\hline 2 & Jeff & Application Analyst & Jus 2 & 5 & & $x$ & \\
\hline 2 & Sally & $\begin{array}{l}\text { Application } \\
\text { Development Manager }\end{array}$ & Jus2 & & $\mathrm{x}$ & $x$ & \\
\hline 2 & Pam & Business Analyst & Jus3 & 0.6 & & $\mathrm{x}$ & \\
\hline 3 & Brendan & Not Stated & Ind1 & 2 & & $\mathrm{x}$ & \\
\hline 3 & Tony & Principal Analyst & Justice 5 & 16 & $\mathrm{x}$ & $\mathrm{x}$ & \\
\hline 3 & Matthew & Senior Advisor & Justice1 & 0.7 & & $\mathrm{x}$ & $x$ \\
\hline 3 & Grant & Independent Advisor & Justice4 & 4 & & $\mathrm{x}$ & \\
\hline 3 & Rebecca & Team Leader & Justice 1 & 0.8 & $\mathrm{x}$ & $\mathrm{x}$ & \\
\hline 3 & Bruce & Chief Analyst & Justice1 & 13 & & $\mathrm{x}$ & \\
\hline 3 & Angela & Senior Policy Advisor & Justice1 & 5 & & $\mathrm{x}$ & \\
\hline 3 & Katy & Legal Adviser & Justice 2 & 12 & & $\mathrm{x}$ & $\mathrm{x}$ \\
\hline 3 & Jackie & Administrator & Justice 1 & 0.3 & & $\mathrm{x}$ & \\
\hline 3 & Molly & Senior Advisor & Justice1 & 2 & & $\mathrm{x}$ & $\mathrm{x}$ \\
\hline 3 & Justin & Legal Adviser & Justice1 & 2.5 & & $\mathrm{x}$ & \\
\hline 3 & John & Principal Advisor & Justice1 & 3 & & $\mathrm{x}$ & \\
\hline 3 & Mike & Independent Advisor & Ind2 & & & $\mathrm{x}$ & \\
\hline 3 & Diana & Senior Policy Advisor & Justice 3 & & $x$ & $x$ & \\
\hline
\end{tabular}




\begin{tabular}{|c|c|c|c|c|c|c|c|}
\hline Case & Name & Role & Organisation & $\begin{array}{l}\text { Tenure in } \\
\text { Organisation } \\
\text { (years) }\end{array}$ & $\begin{array}{c}\text { Phase } \\
1 \\
\end{array}$ & $\begin{array}{c}\text { Phase } \\
2 \\
\end{array}$ & $\begin{array}{c}\text { Phase } \\
\mathbf{3} \\
\end{array}$ \\
\hline 4 & Alexa & Strategy Manager & Health2 & 1.10 & $\mathrm{x}$ & & \\
\hline 4 & Anthony & $\begin{array}{l}\text { Manager, Performance } \\
\text { Outcomes }\end{array}$ & Health1 & 15.00 & $\mathrm{x}$ & & \\
\hline 4 & Ashley & Programme Manager & Health4 & 3.50 & & & \\
\hline 4 & Bevan & $\begin{array}{l}\text { Manager, System } \\
\text { Performance }\end{array}$ & Health1 & 8.00 & & & $\mathrm{x}$ \\
\hline 4 & Carl & $\begin{array}{l}\text { Manager, Information } \\
\text { \& Analysis }\end{array}$ & Support3 & 4.00 & & & \\
\hline 4 & Charles & Analyst/Programmer & Health2 & 0.10 & & & \\
\hline 4 & Chris & Specialist Leader & Health3 & 6.00 & & & \\
\hline 4 & Ewan & Analyst & Support4 & 2.00 & & & \\
\hline 4 & Hannah & Performance Analyst & Support2 & 0.60 & & & \\
\hline 4 & James & $\begin{array}{l}\text { Business Intelligence } \\
\text { Analyst }\end{array}$ & Support3 & 2.00 & & & \\
\hline 4 & Jane & Operations Manager & Health2 & 0.60 & & & $x$ \\
\hline 4 & Kirsten & Information Analyst & Support4 & 2.00 & & & \\
\hline 4 & Mark & Chief Advisor & Health1 & 3.00 & & & \\
\hline 4 & Patricia & Programme Analyst & Health2 & 1.00 & & & \\
\hline 4 & Patrick & Technical Lead & Health2 & 0.40 & & & \\
\hline 4 & Paul & Analyst & Support1 & 1.00 & & & \\
\hline 4 & Penny & Chief Advisor & Health1 & 16.00 & & & \\
\hline 4 & Robin & Performance Analyst & Support2 & 2.00 & & & \\
\hline 4 & Stephanie & Financial Analyst & Support3 & 5.00 & & & \\
\hline 4 & Susan & Senior Analyst & Support1 & 5.00 & & & \\
\hline
\end{tabular}




\section{Appendix 9: Participant's perceptions of knowledge}

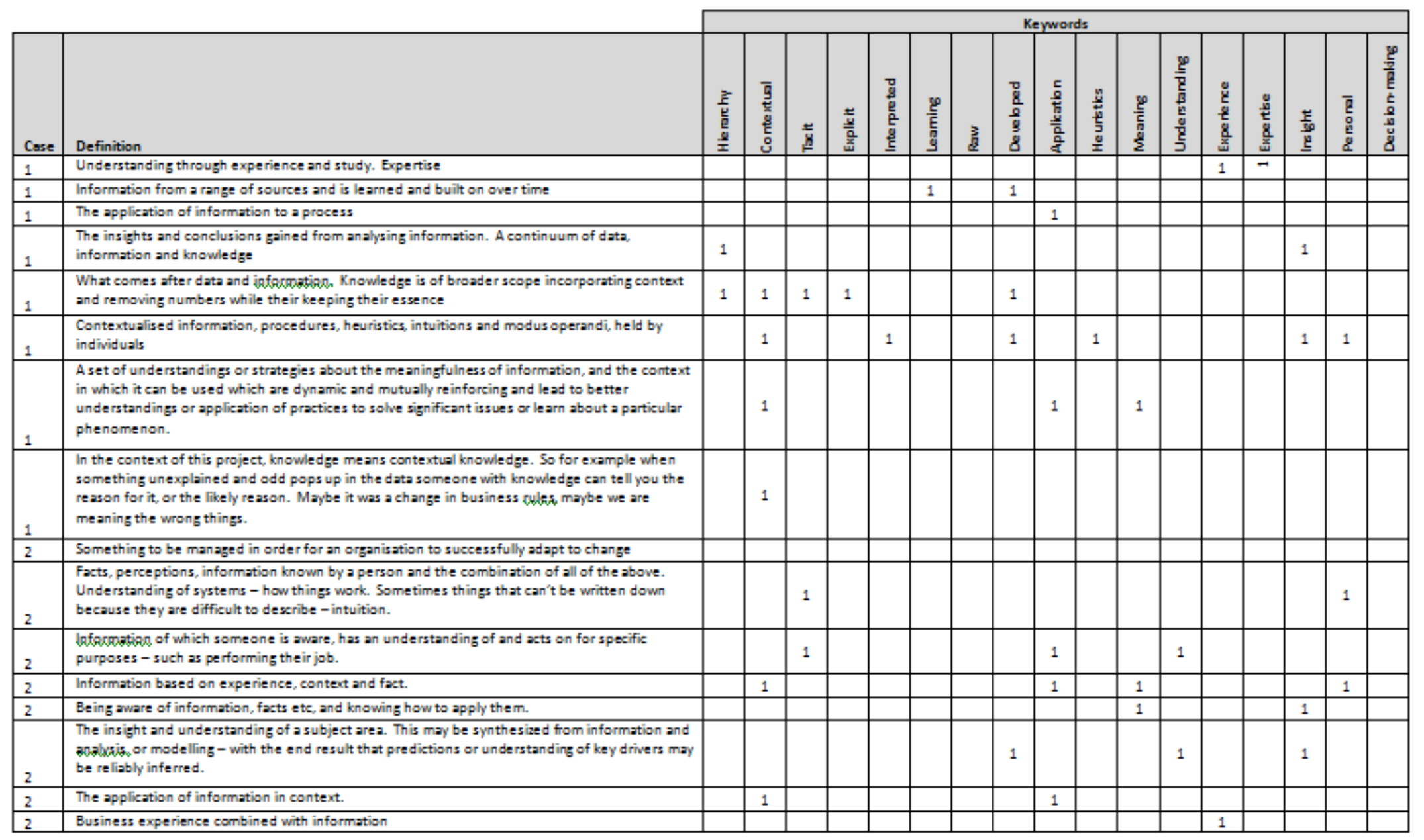




\begin{tabular}{|c|c|c|c|c|c|c|c|c|c|c|c|c|c|c|c|c|c|c|}
\hline \multirow[b]{2}{*}{ Cose } & \multirow[b]{2}{*}{ Definition } & \multicolumn{17}{|c|}{ Keywords } \\
\hline & & \begin{tabular}{|c|}
$\vec{E}$ \\
$\underline{E}$ \\
$\frac{g}{I}$ \\
\end{tabular} & 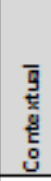 & $\frac{\pi}{n}$ & 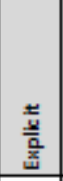 & 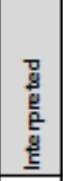 & 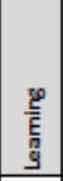 & 童 & \begin{tabular}{|l}
7 \\
$\frac{8}{2}$ \\
$\frac{9}{3}$ \\
8 \\
\end{tabular} & $\begin{array}{l}c \\
\frac{8}{1} \\
\frac{5}{0} \\
\frac{a}{2}\end{array}$ & 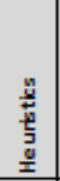 & 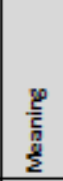 & 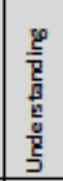 & \begin{tabular}{l|l}
$\frac{8}{8}$ \\
$\frac{8}{8}$ \\
$\frac{8}{3}$ \\
\end{tabular} & 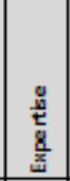 & $\begin{array}{l}\underline{\underline{F}} \\
\underline{\underline{\underline{E}}} \\
\underline{\underline{\underline{E}}} \\
\end{array}$ & $\begin{array}{l}\overline{2} \\
\vdots \\
\frac{1}{2} \\
\end{array}$ & 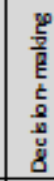 \\
\hline 3 & $\begin{array}{l}\text { An understanding of how things are done, why things are like they are how to go about } \\
\text { achieving objectives. }\end{array}$ & & & & & & & & & & & & 1 & & & & & \\
\hline 3 & $\begin{array}{l}\text { The ability to transfer data into information relevant for decision and the ability to make } \\
\text { decisions that guide future actions, using learning and experience }\end{array}$ & & & & & & 1 & & & 1 & & & & 1 & & & & 1 \\
\hline 3 & $\begin{array}{l}\text { Information that has more than intrinsic value and is capable of being correctly applied for } \\
\text { prastical purposes. }\end{array}$ & & & & & & & & & 1 & & & & & & & & \\
\hline 3 & $\begin{array}{l}\text { A combination of factual information recalled; understanding of how things (system or machines } \\
\text { etc) work; understanding about how to do things, es research; knowing where to find } \\
\text { information/who to ask. }\end{array}$ & & & & & & & & & & & & 1 & & & & & \\
\hline 3 & $\begin{array}{l}\text { Being aware of relevant information in the area, having an idea of how to conceptualise the } \\
\text { connections and judging the relative importance of information in a particular application. }\end{array}$ & & & & & 1 & & & & 1 & & & & & & & & \\
\hline 3 & What you know about a subject matters as area of expertise. & & & & & & & & & & & & & & 1 & & 1 & \\
\hline 3 & $\begin{array}{l}\text { A combination of information and experience not necessarily documented in one place. } \\
\text { Knowiedge requires ability of a person to communicate relevant information in context in a } \\
\text { meaningful way so that others may use it or be able to understand it. }\end{array}$ & & 1 & 1 & & & & & & 1 & & 1 & 1 & 1 & & & 1 & \\
\hline 3 & Knowledge is the ability to understand, use and contextualise information. & & 1 & & & & & & & 1 & & & 1 & & & & & \\
\hline 3 & $\begin{array}{l}\text { Information learned through rese arch over a period of time resulting in a body of knowiedge } \\
\text { remembered. }\end{array}$ & & & & & & 1 & & & & & & & 1 & 1 & & & \\
\hline 3 & $\begin{array}{l}\text { The set of understand ings I have about the Ministry, government processes, the law - the thing } \\
\text { that give me a context for this project. }\end{array}$ & & 1 & & & & & & & & & & & 1 & & & 1 & \\
\hline 3 & The ability to answer questions on a subject or to know where to find answers. & & & & & & & & & & & & & 1 & 1 & & & \\
\hline 3 & A detailed understanding derived from information and experience & & & & & & & & & & & & 1 & 1 & & & & \\
\hline 3 & Understanding of the social and institutional contert through which information flows & & 1 & & & & & & & & & & & & & & & \\
\hline & $\begin{array}{l}\text { Internalised capability to take decisions and actions in a way that may be tacit and difficult to } \\
\text { codify }\end{array}$ & & & 1 & & & & & & 1 & & & & & & & 1 & 1 \\
\hline
\end{tabular}




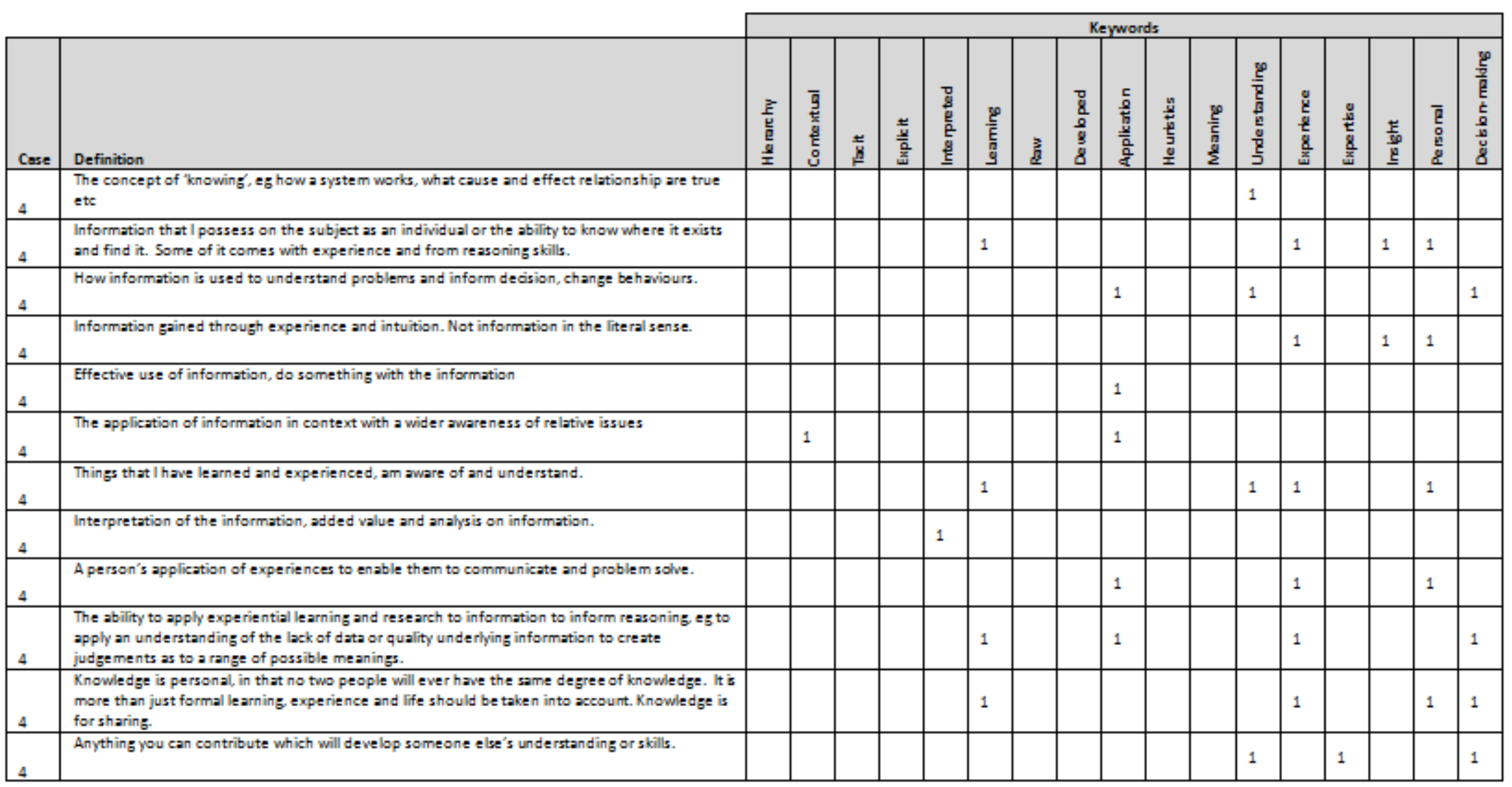


Appendix 10: Examples of Case 1 survey data
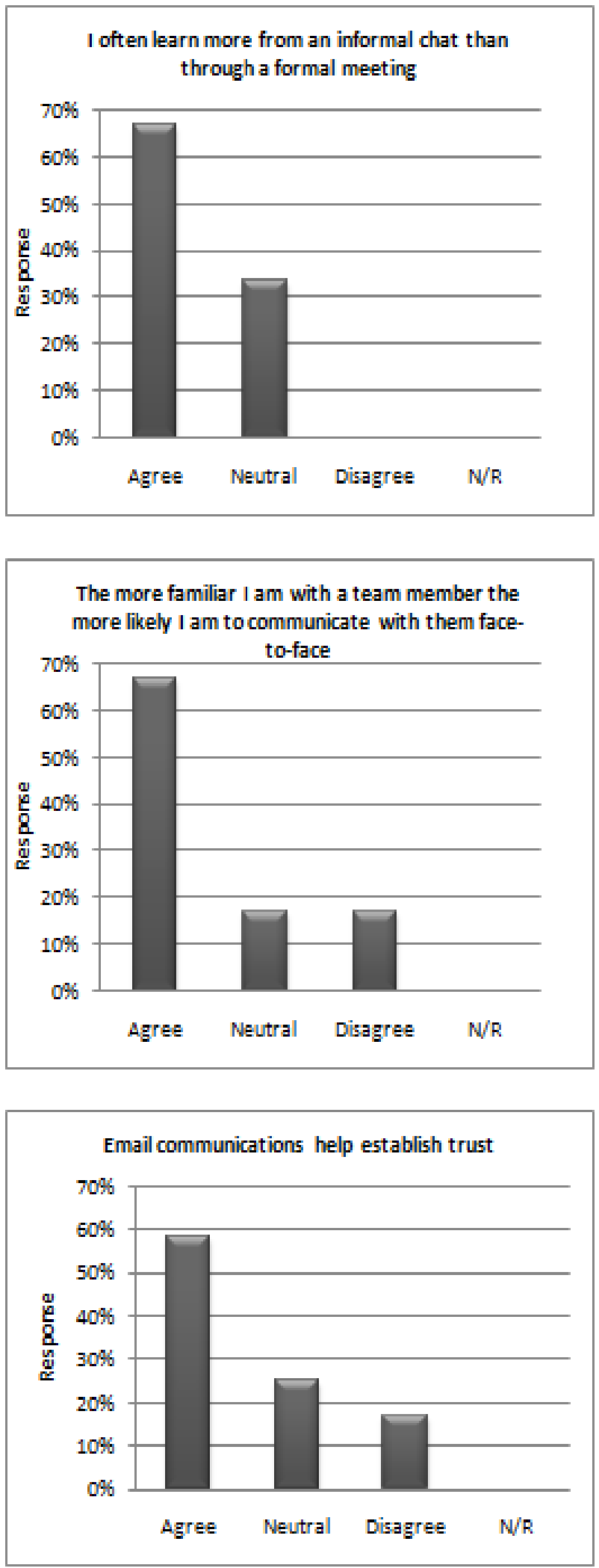

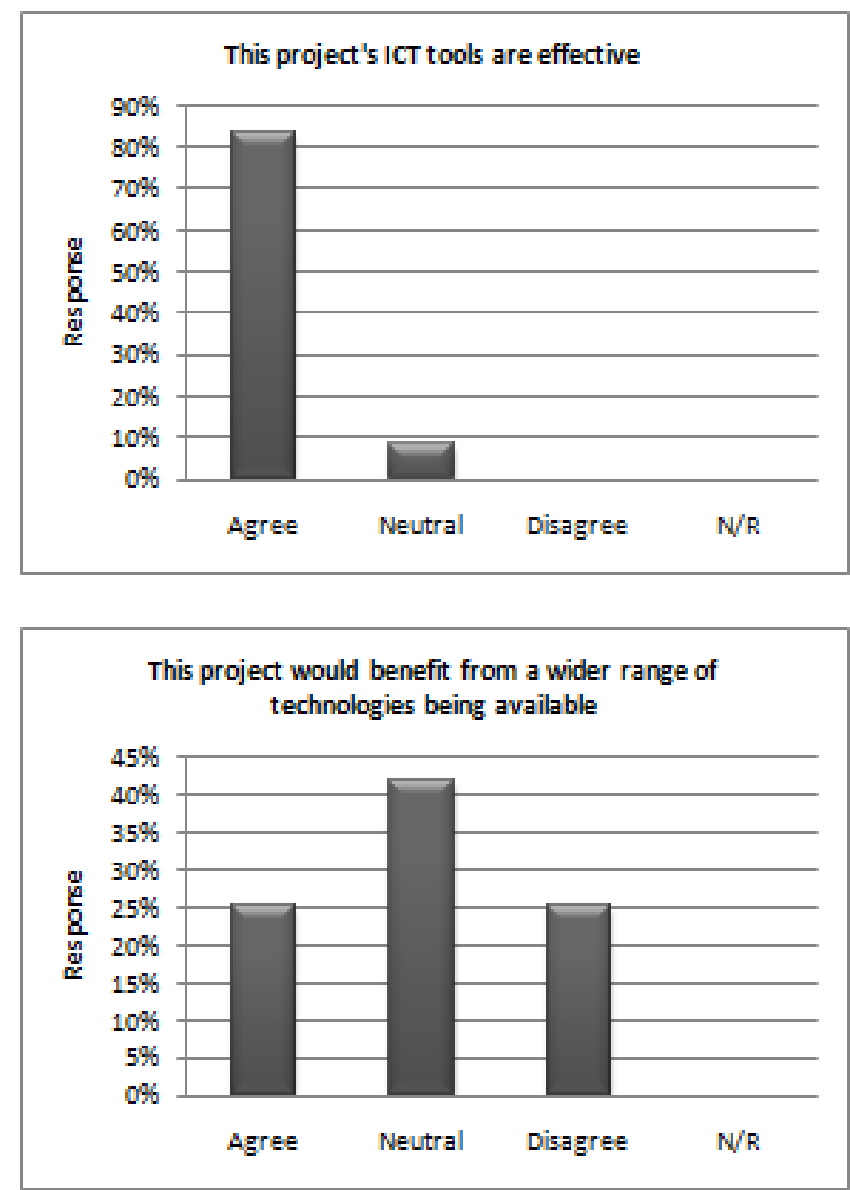

My parent organisation places a strong emphais on using technology to share information

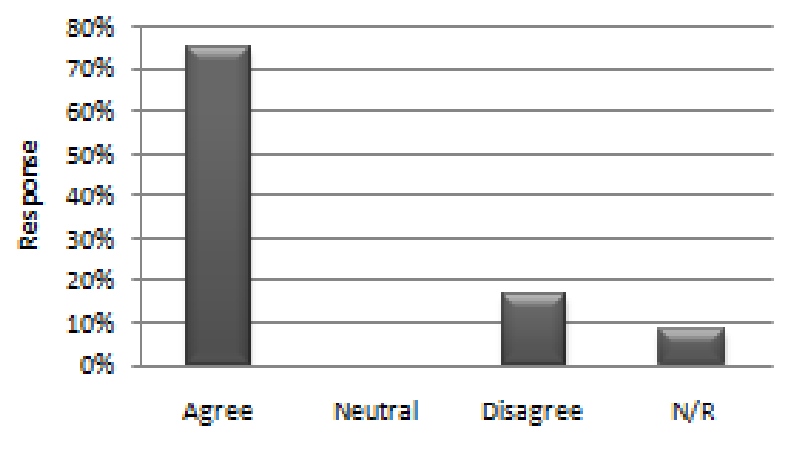



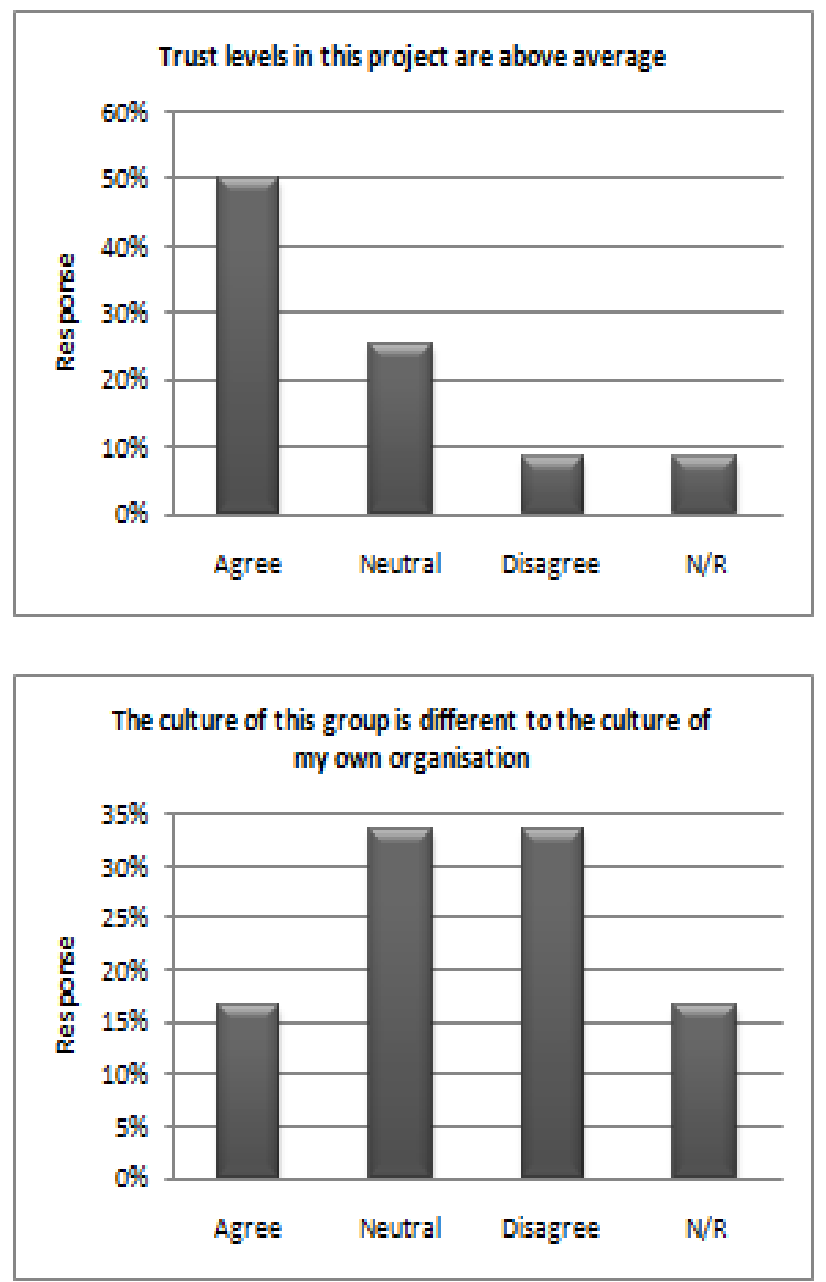
Appendix 11: Case 1 Clique Analysis (from UCINET)

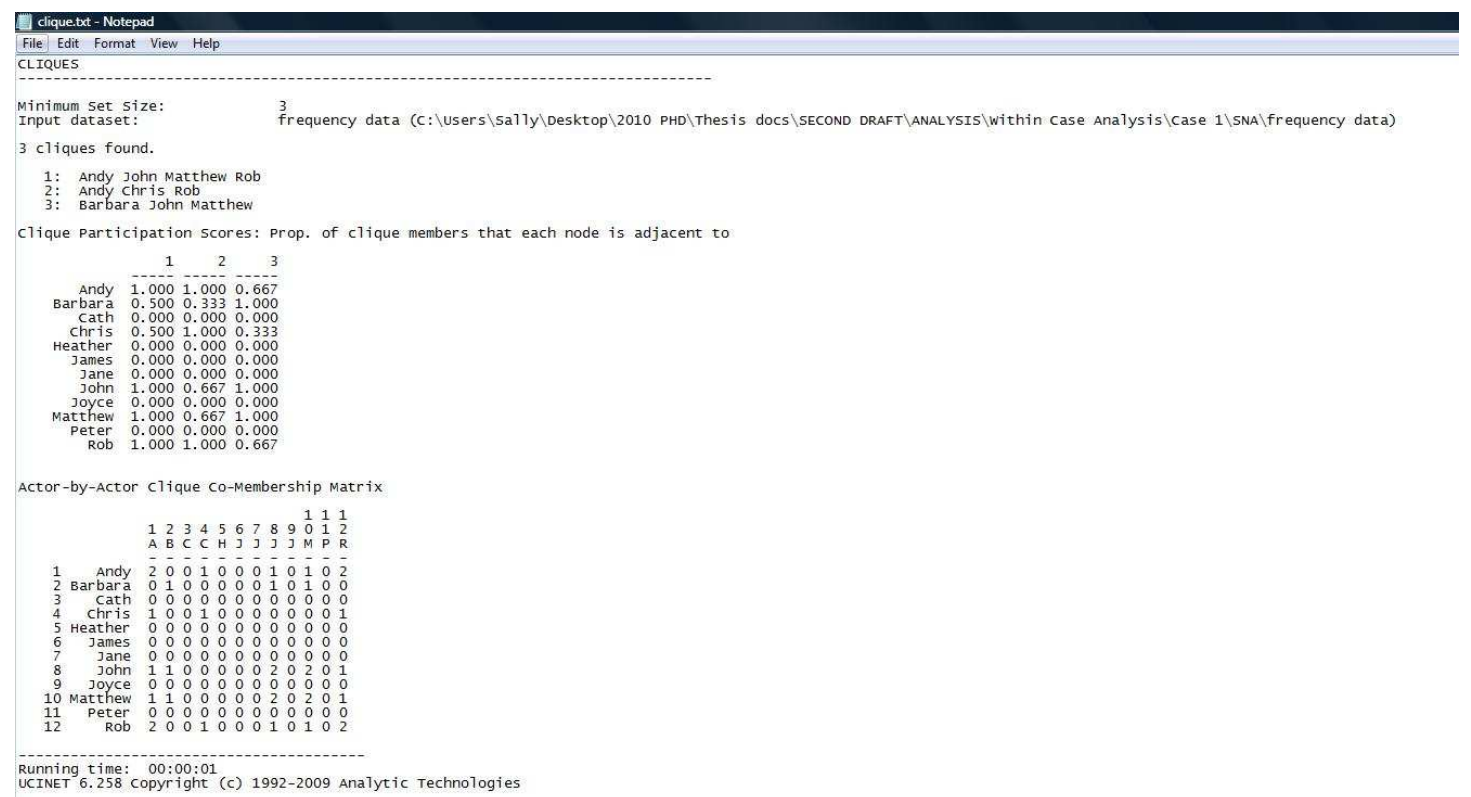


Appendix 12: Examples of Case 2 survey data
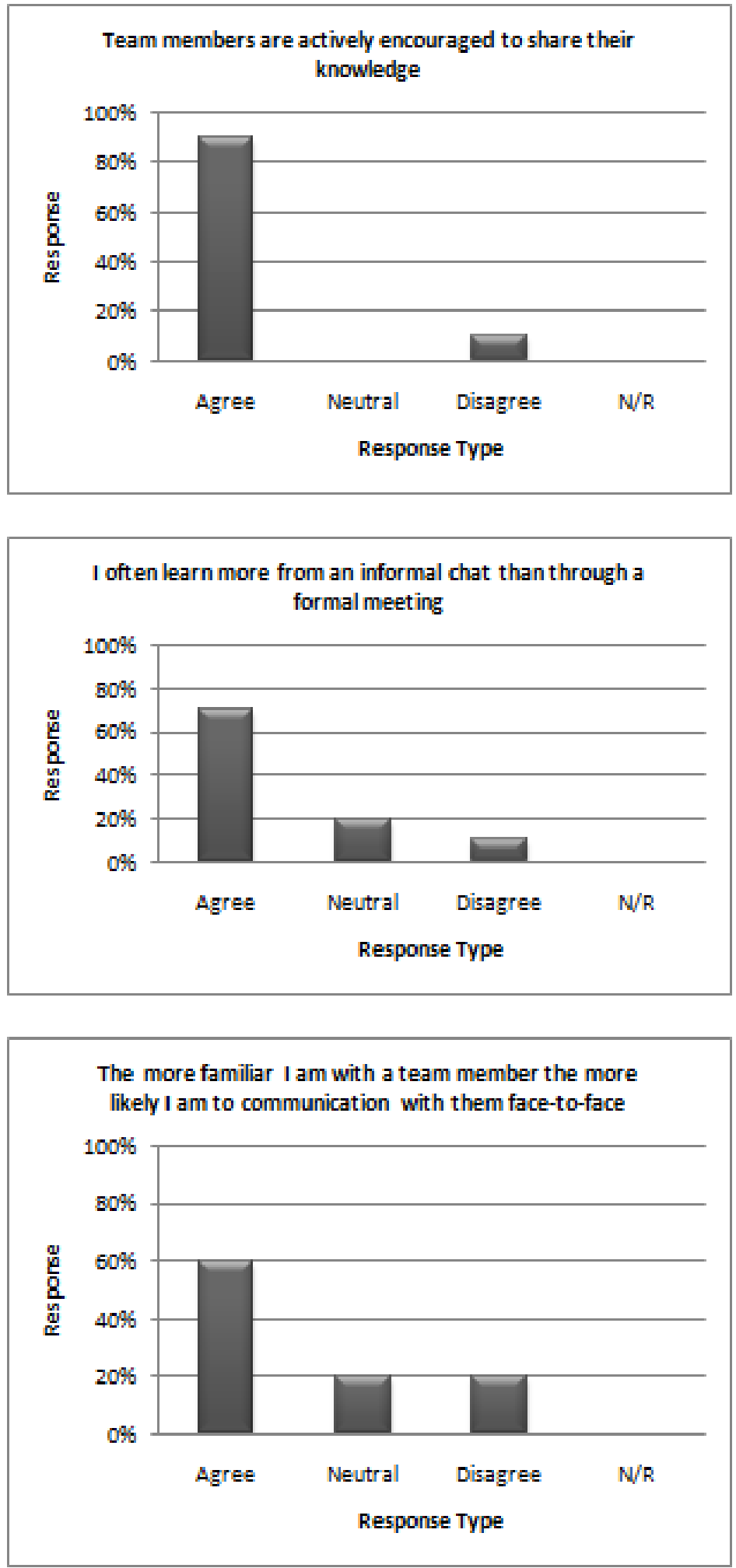

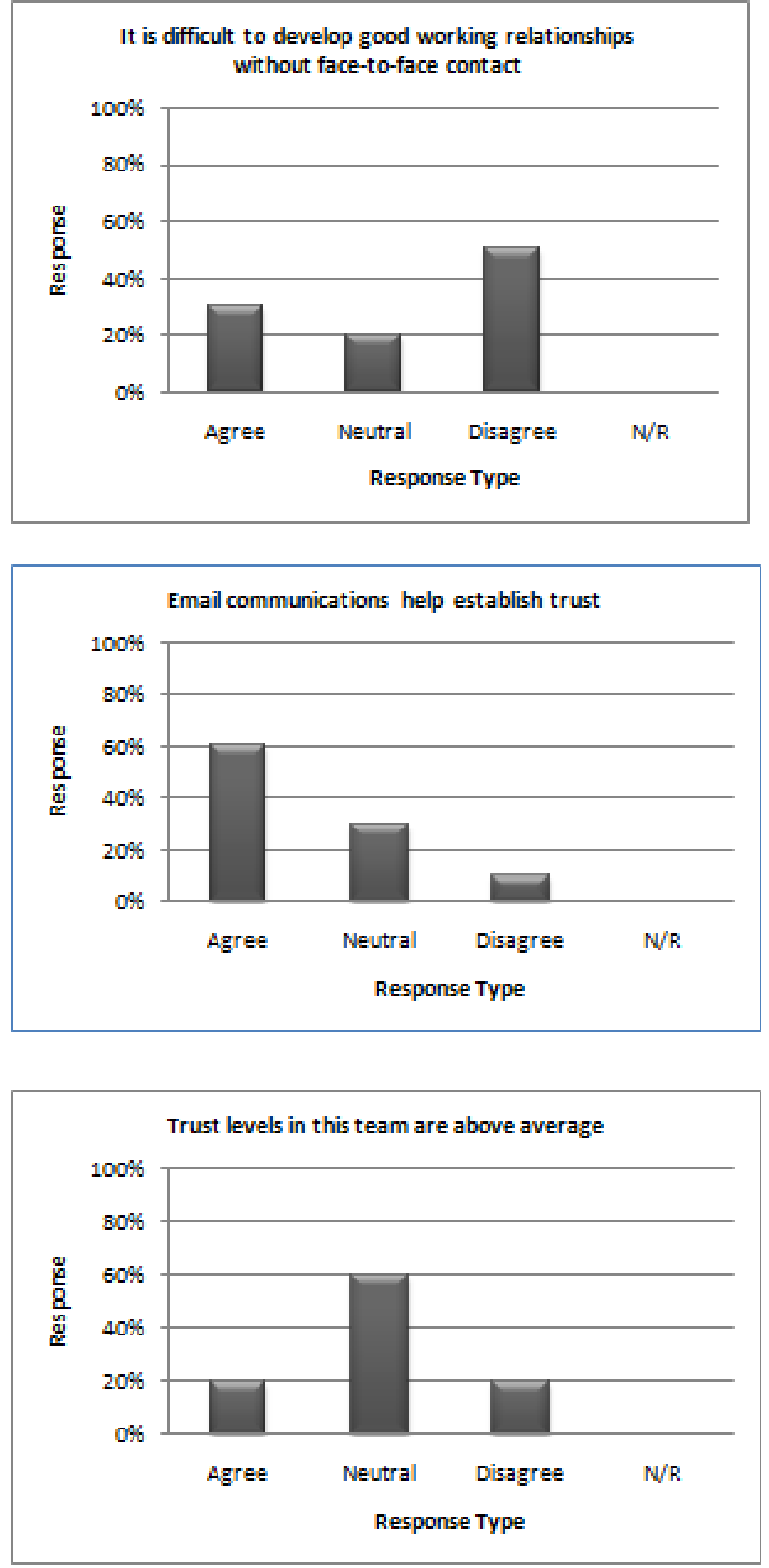

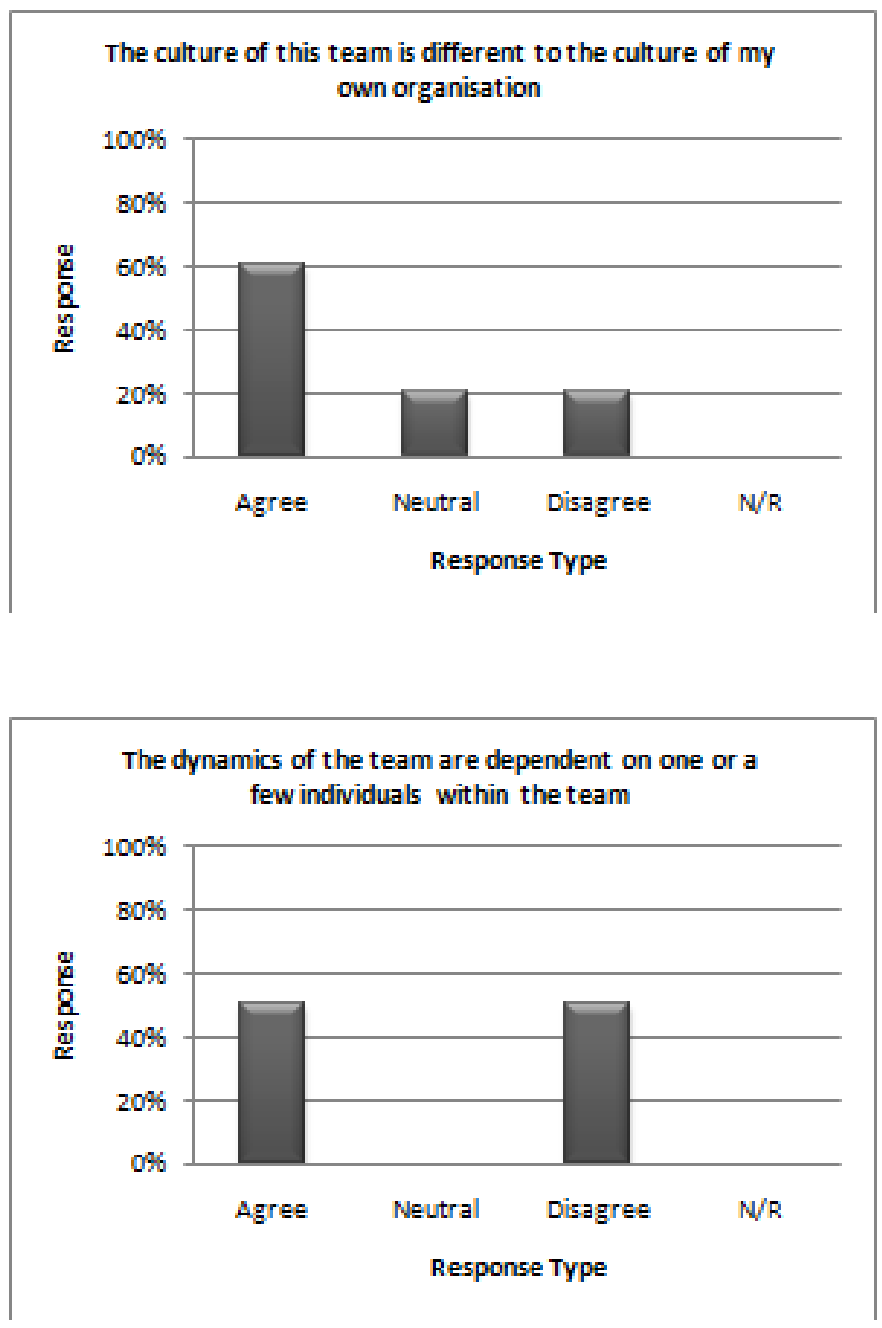
Appendix 13: Examples of Case 3 survey data
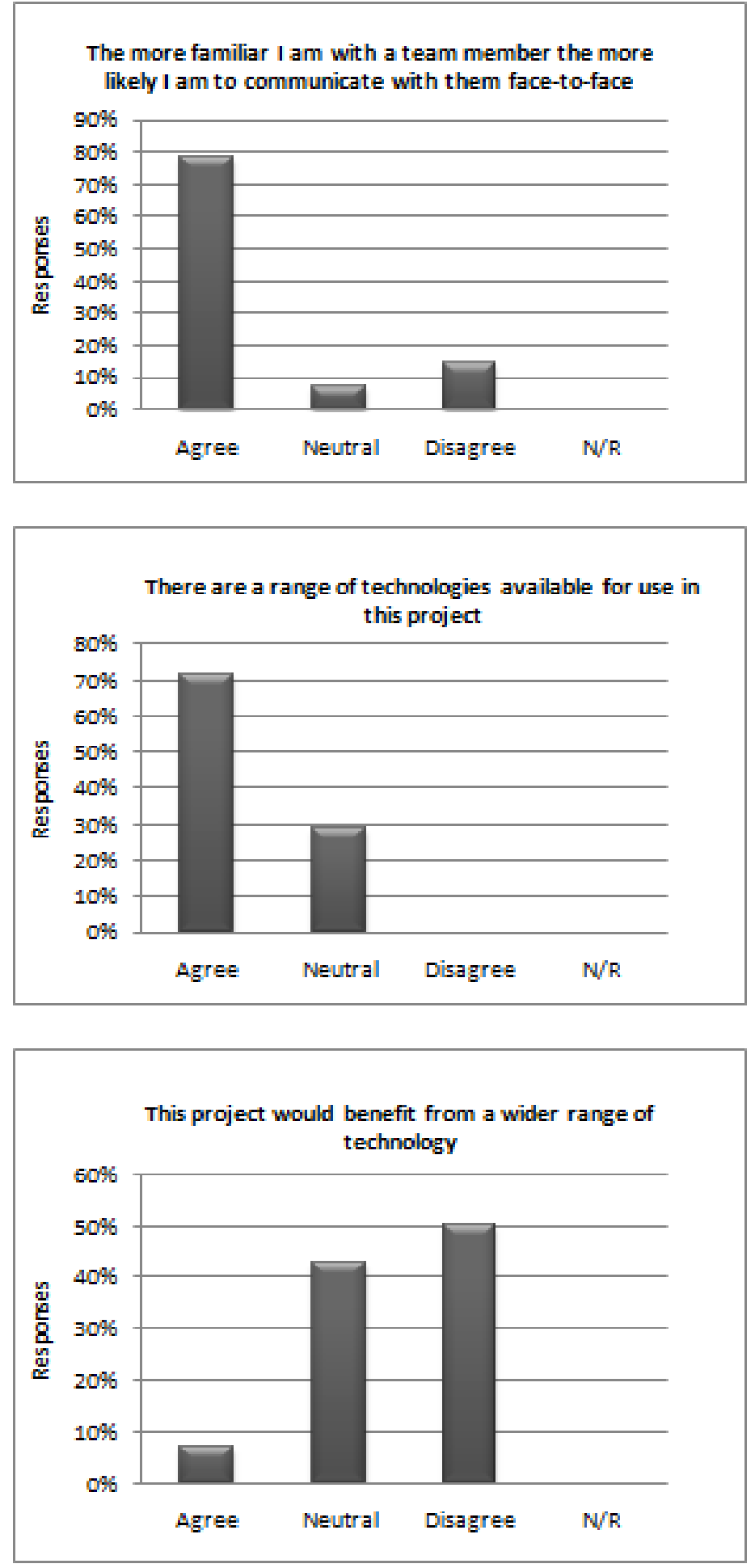

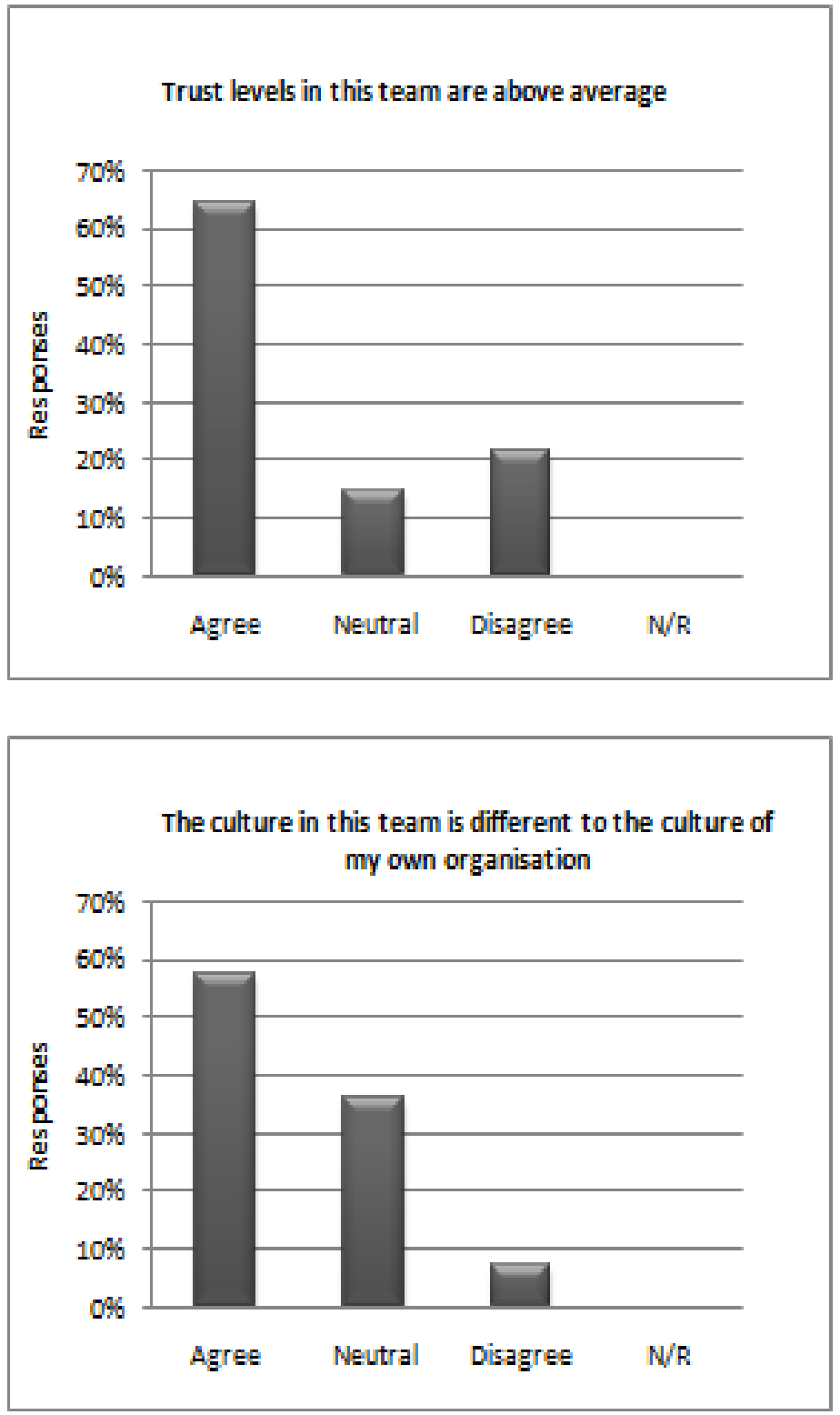


\section{Appendix 14: Example of Case 3 SNA centrality \& clique data}

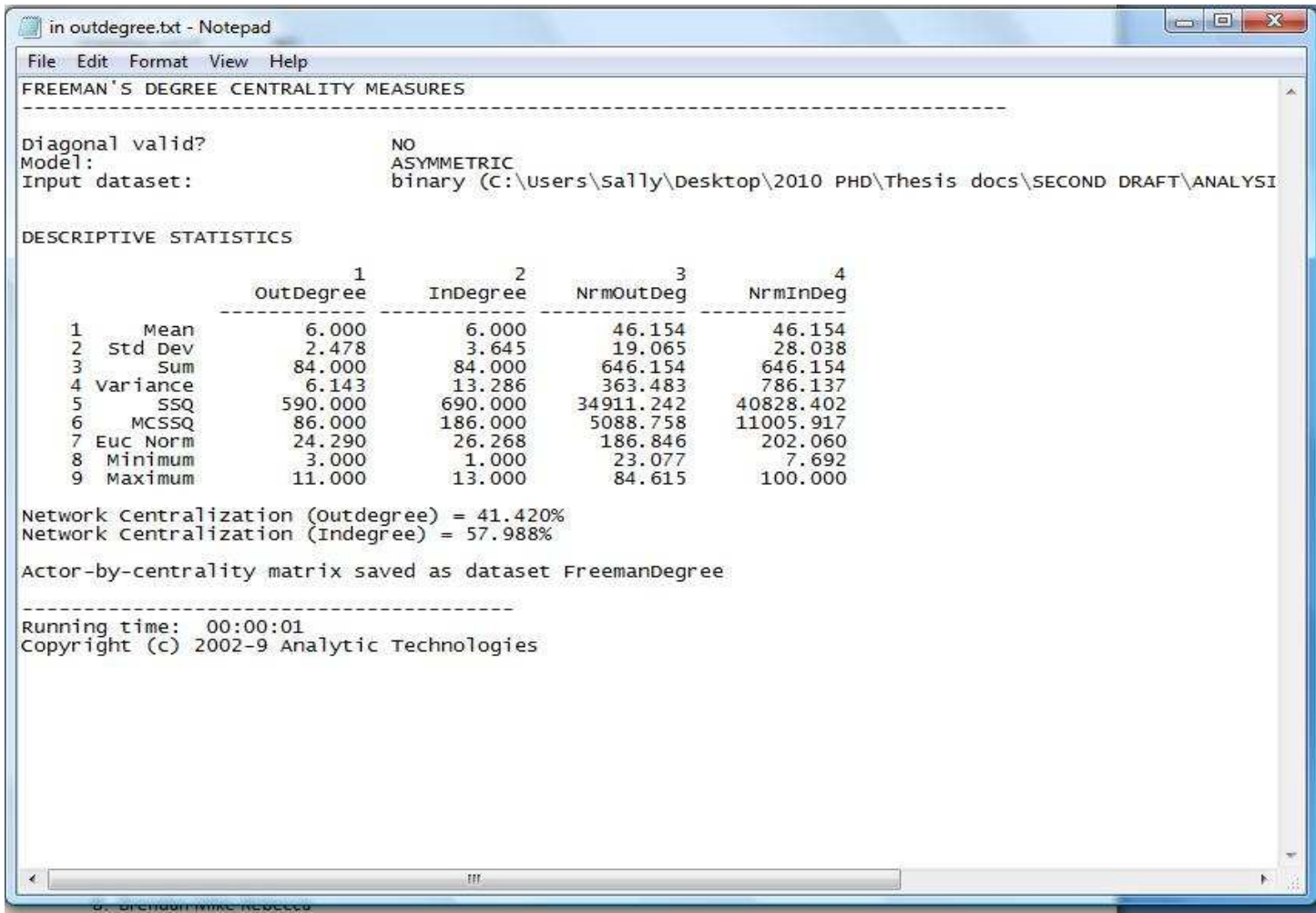
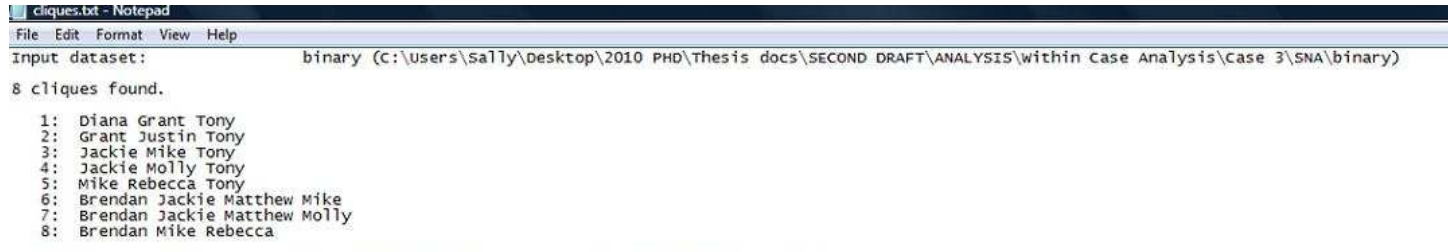

clique participation scores: Prop. of clique members that each node is adjacent to

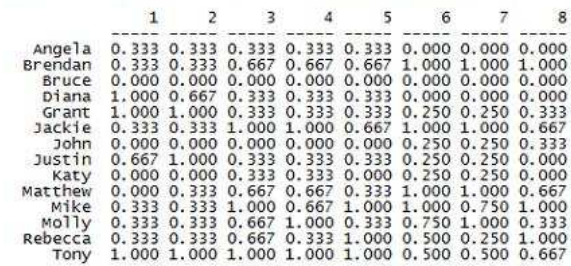

Actor-by-Actor Clique co-membership Matrix

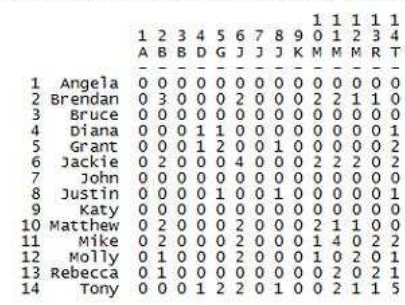

Running time: $00: 00: 01$
Output generated: 19 May $1014: 39: 17$
UCINET 6.258 Copyright
(c) $1992-2009$ Analytic Technologies 
Appendix 15: Examples of Case 4 survey data
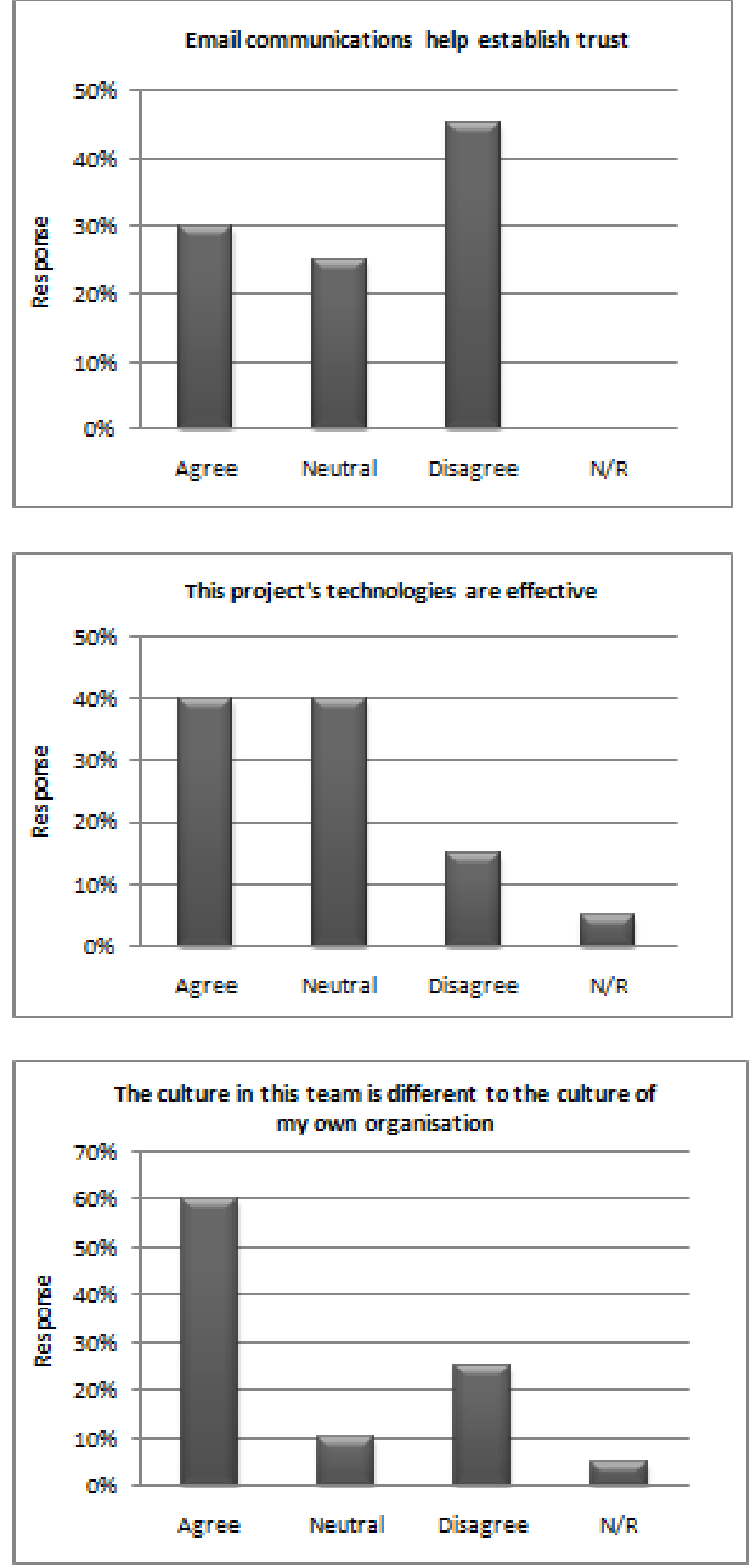


\section{Appendix 16: Frequency of Communication/Proximity}

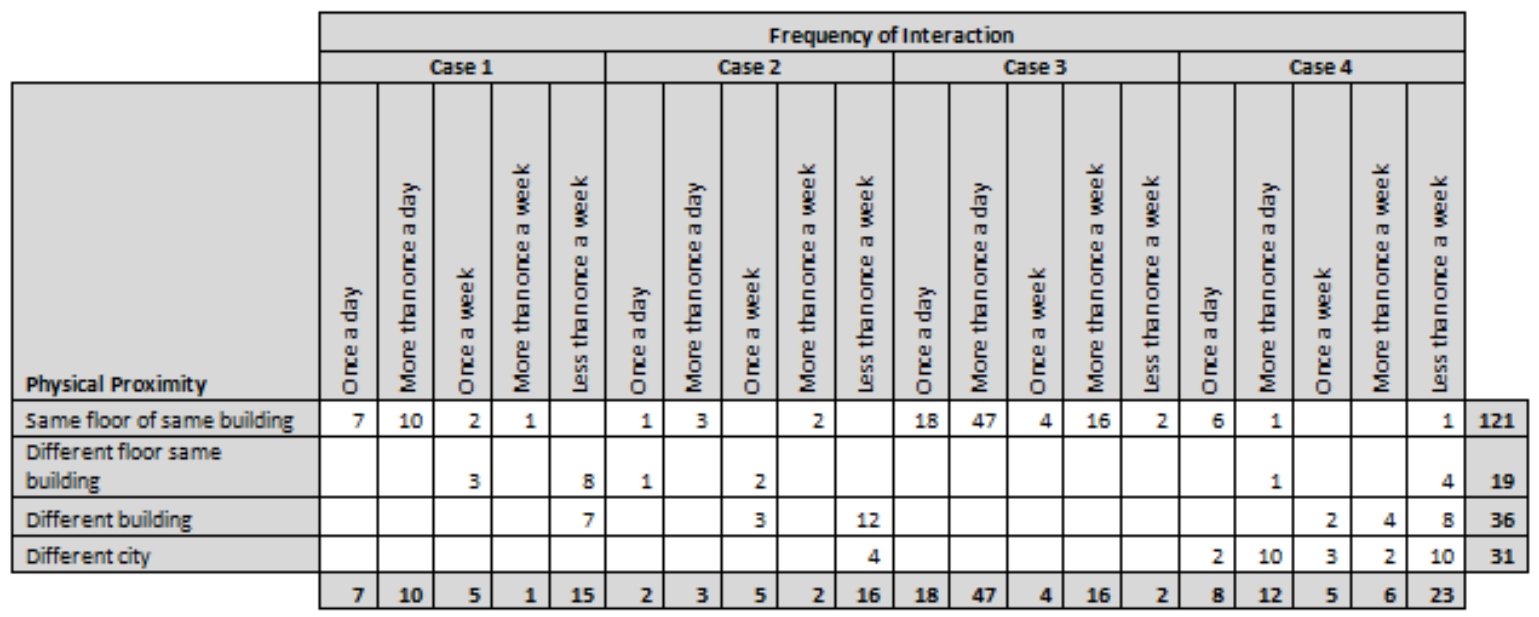




\section{Appendix 17: Examples of cross-case analysis data}
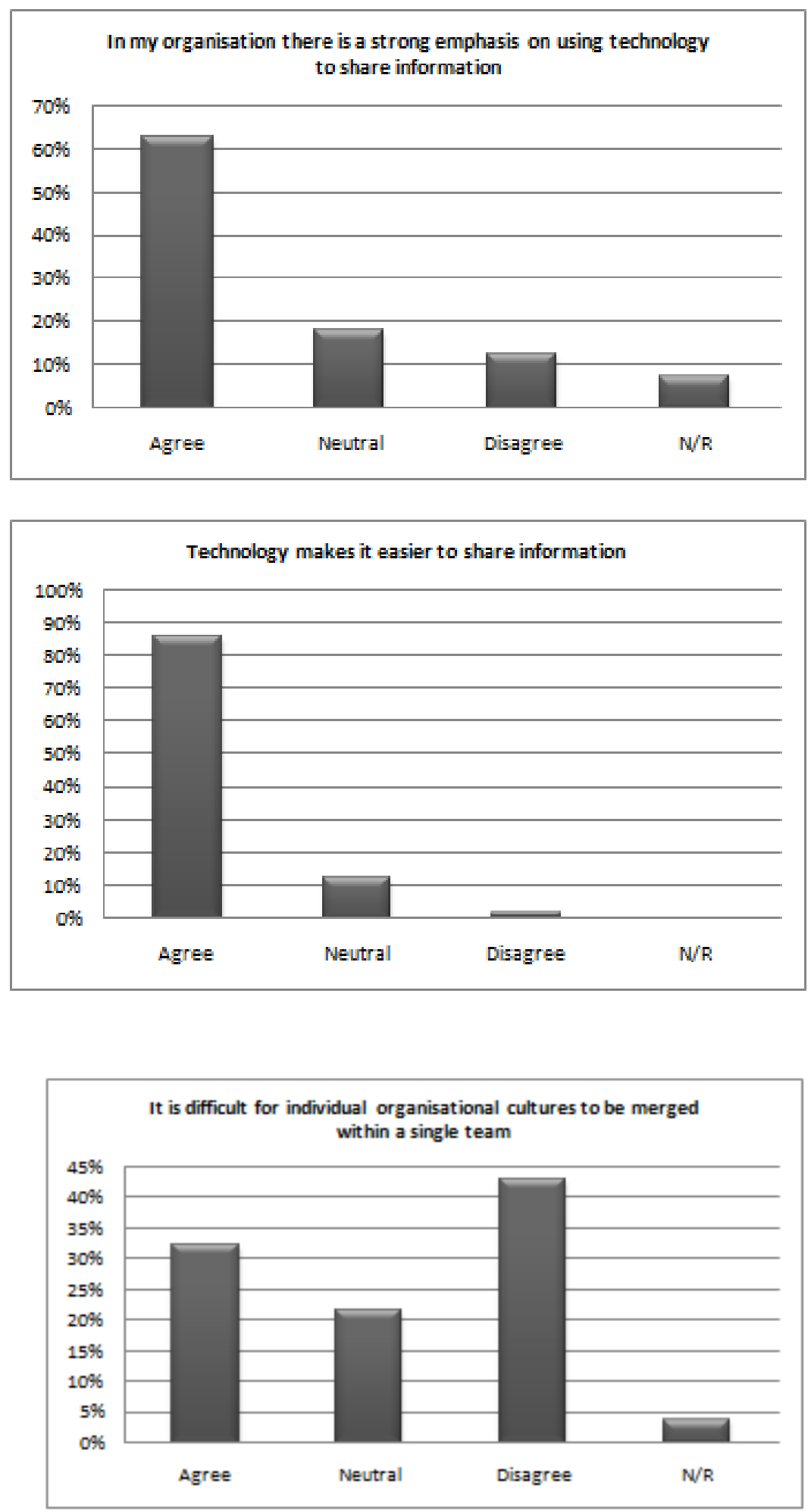


\section{Appendix 18: Generic Knowledge Sharing Barriers}

\begin{tabular}{|c|c|c|c|c|c|c|c|c|c|c|c|c|c|c|c|c|c|c|c|c|c|}
\hline \multicolumn{2}{|c|}{$e^{2}$} & \multicolumn{20}{|c|}{ Keywords } \\
\hline ID & Barrier & 蒙 & 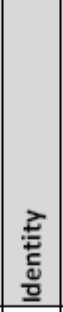 & 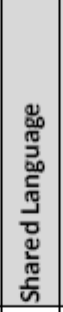 & 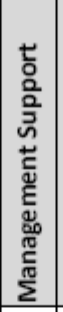 & 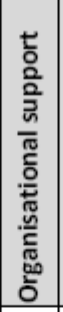 & 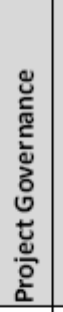 & 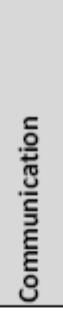 & $\stackrel{\text { ह̆ }}{\underline{F}}$ & $\begin{array}{l}\frac{\vec{z}}{\underline{E}} \\
\frac{1}{x} \\
\frac{0}{2} \\
\end{array}$ & 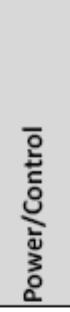 & 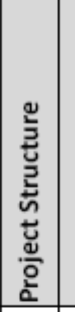 & 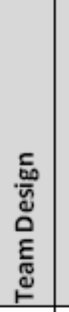 & 党 & $\begin{array}{l}\text { है } \\
\text { 힐 }\end{array}$ & 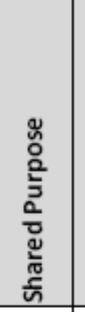 & 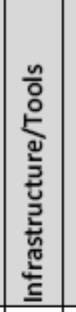 & 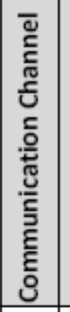 & 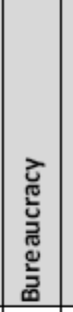 & 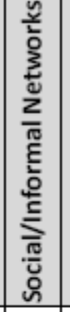 & 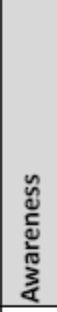 \\
\hline A1 & Sign off from senior management to share information & & & & & 1 & & & & & & & & & & & & & & & \\
\hline \multirow[t]{4}{*}{ A2 } & Poor communication & & & & & & & 1 & & & & & & & & & & & & & \\
\hline & Lack of project structure & & & & & & & & & & & 1 & & & & & & & & & \\
\hline & Unclear terms of reference & & & & & & & & & & & 1 & & & & & & & & & \\
\hline & Lack of communication & & & & & & & 1 & & & & & & & & & & & & & \\
\hline \multirow[t]{2}{*}{ A3 } & Individual unwillingness to share & & & & & & & & & & 1 & & & & & & & & & & \\
\hline & Not using shared information tools & & & & & & & & & & & & & & & & 1 & & & & \\
\hline \multirow[t]{2}{*}{ A4 } & Knowing who knows what & & & & & & & & & & & & & & & & & & & & 1 \\
\hline & Time pressures & & & & & & & & 1 & & & & & & & & & & & & \\
\hline \multirow[t]{3}{*}{ A5 } & Knowledge is power attitude & & & & & & & & & & 1 & & & & & 1 & & & & & \\
\hline & Selfishness & & & & & & & & & & 1 & & & & & & & & & & \\
\hline & Intractability & & & & & & & & & & 1 & & & & & & & & & & \\
\hline \multirow[t]{3}{*}{ A6 } & Incompetent or self-serving management & & & & & 1 & & & & & & & & & & & & & & & \\
\hline & Illogical/irrational decision making & & & & & & 1 & & & & & & & & & & & & & & \\
\hline & Overly-rigid bureaucratic processes & & & & & 1 & & & & & & & & & & & & & 1 & & \\
\hline \multirow[t]{2}{*}{ A8 } & Time pressures (lack of time to engage with others) & & & & & & & & 1 & & & & & & & & & & & & \\
\hline & $\begin{array}{l}\text { Constant deadlines for reactive requests, meaning urgency comes before } \\
\text { importance }\end{array}$ & & & & & & & & 1 & & & & & & & & & & & & \\
\hline \multirow[t]{3}{*}{ A9 } & Lack of physical proximity & & & & & & & & & 1 & & & & & & & & & & & \\
\hline & $\begin{array}{l}\text { CEOs telling you you're not allowed to share even when you've got an } \\
\text { agreement in place }\end{array}$ & & & & & 1 & & & & & & & & & & & & & & & \\
\hline & Lack of support from management & & & & & 1 & & & & & & & & & & & & & & & \\
\hline \multirow[t]{2}{*}{ A10 } & $\begin{array}{l}\text { Prioritisation - when different members of the group prioritise sharing } \\
\text { differently }\end{array}$ & & & & & & & & & & & & & & & 1 & & & & & \\
\hline & Time & & & & & & & & 1 & & & & & & & & & & & & \\
\hline
\end{tabular}




\begin{tabular}{|c|c|c|c|c|c|c|c|c|c|c|c|c|c|c|c|c|c|c|c|c|c|}
\hline \multirow[b]{2}{*}{ ID } & \multirow[b]{2}{*}{ Barrier } & \multicolumn{20}{|c|}{ Keywords } \\
\hline & & 萤 & 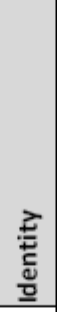 & 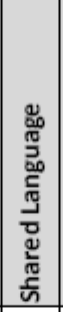 & 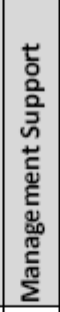 & 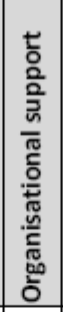 & 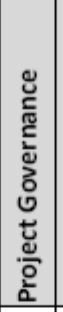 & 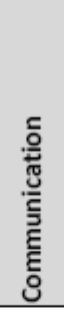 & $\underset{\xi}{\tilde{F}}$ & 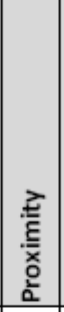 & 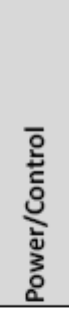 & 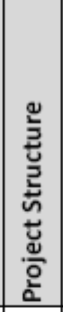 & 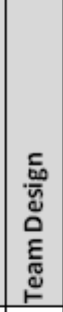 & 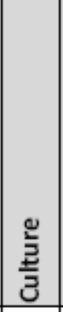 & \begin{tabular}{|l}
$\tilde{y}$ \\
हे \\
$z$
\end{tabular} & 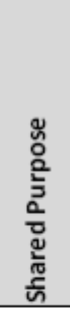 & 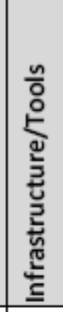 & 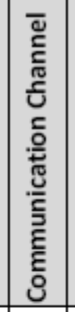 & 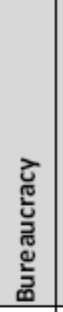 & 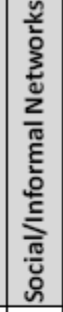 & 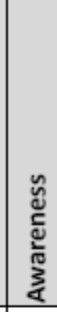 \\
\hline \multirow[t]{3}{*}{ B1 } & Knowing who knows what & & & & & & & & & & & & & & & & & & 1 & & \\
\hline & Trusting enough to share & 1 & & & & & & & & & & & & & & & & & & & \\
\hline & Having enough time together & & & & & & & & 1 & & & & & & & & & & & & \\
\hline \multirow[t]{4}{*}{ B2 } & Lack of trust & 1 & & & & & & & & & & & & & & & & & & & \\
\hline & Access to appropriate technologies & & & & & & & & & & & & & & & & 1 & & & & \\
\hline & Varying degrees of individual commitment & & & & & & & & & & & & & & & & & & & & 1 \\
\hline & Varying degrees of organisational commitment & & & & & 1 & & & & & & & & & & & & & & & 1 \\
\hline \multirow[t]{3}{*}{ B3 } & $\begin{array}{l}\text { Individual's reluctance to share knowledge with people from another } \\
\text { organisation who they don't know well }\end{array}$ & 1 & & & & & & & & & & & & & & & & & & & \\
\hline & Availability of individuals & & & & & & & & 1 & & & & & & & & & & & & \\
\hline & Technological compatibility & & & & & & & & & & & & & & & & 1 & & & & \\
\hline B4 & Communication (inconsistent/lack of) & & & & & & & 1 & & & & & & & & & & & & & \\
\hline \multirow[t]{2}{*}{ B5 } & Individual willingness to share & & & & & & & & & & 1 & & & & & & & & & & \\
\hline & Ignorance of how information will be used by others & 1 & & & & & & & & & & & & & & & & & & & \\
\hline \multirow[t]{2}{*}{ B6 } & Unwillingness to share & & & & & & & & & & 1 & & & & & & & & & & \\
\hline & Individual levels of competency and expertise & & & & & & & & & & & & & & & & & & & & 1 \\
\hline \multirow[t]{2}{*}{ B7 } & Individual's level of commitment & & & & & & & & & & & & & & & & & & & & 1 \\
\hline & Lack of recognition for sharing & & & & & & & & & & & & & & & & & & & 1 & \\
\hline \multirow[t]{3}{*}{ B8 } & Issues of confidentiality & 1 & & & & & & & & & & & & & & & & & & & \\
\hline & $\begin{array}{l}\text { Competition, not wanting to provide others who could be seen as competitions } \\
\text { for information }\end{array}$ & & & & & & & & & & 1 & & & & & & & & & & \\
\hline & Jargon and acronyms & & & 1 & & & & & & & & & & & & & & & & & \\
\hline B9 & Poor communication & & & & & & & 1 & & & & & & & & & & & & & \\
\hline
\end{tabular}




\begin{tabular}{|c|c|c|c|c|c|c|c|c|c|c|c|c|c|c|c|c|c|c|c|c|c|}
\hline \multirow[b]{2}{*}{ ID } & \multirow[b]{2}{*}{ Barrier } & \multicolumn{20}{|c|}{ Keywords } \\
\hline & & 蒙 & 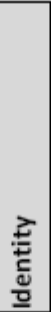 & 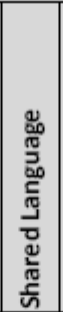 & 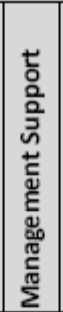 & 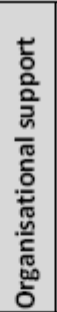 & 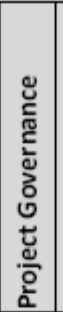 & 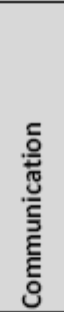 & $\stackrel{\Xi}{\xi}$ & $\begin{array}{l}\frac{\partial}{E} \\
\frac{1}{x} \\
\frac{0}{2} \\
\frac{2}{2}\end{array}$ & 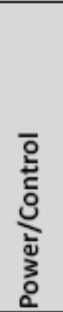 & 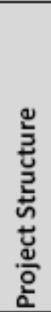 & 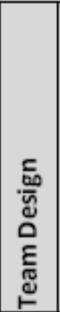 & 音 & है & 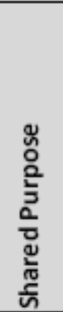 & 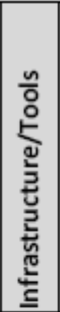 & 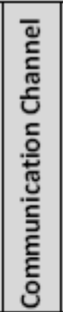 & 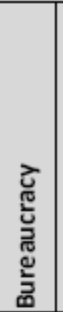 & 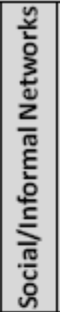 & 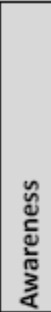 \\
\hline \multirow[t]{3}{*}{ C1 } & Lack of time to explore ideas with others & & & & & & & & 1 & & & & & & & & & & & & \\
\hline & Knowing who knows what & & & & & & & & & & & & & & & & & & 1 & & \\
\hline & Building trust amongst team members & 1 & & & & & & & & & & & & & & & & & & & \\
\hline \multirow[t]{3}{*}{$\mathrm{C} 2$} & Too busy working on day-to-day stuff & & & & & & & & 1 & & & & & & & & & & & & \\
\hline & Not knowing other's skills, knowledge & & & & & & & & & & & & & & & & & & 1 & & \\
\hline & Not knowing other's expectations & & & & & & & & & & & & & & & 1 & & & & & \\
\hline \multirow[t]{3}{*}{$\mathrm{C3}$} & Poor technology & & & & & & & & & & & & & & & & 1 & & & & \\
\hline & Physical separation of team members & & & & & & & & & 1 & & & & & & & & & & & \\
\hline & Lack of trust between team members & 1 & & & & & & & & & & & & & & & & & & & \\
\hline \multirow[t]{2}{*}{$\mathrm{C4}$} & Knowing who knows what & & & & & & & & & & & & & & & & & & 1 & & \\
\hline & Establishing trust & 1 & & & & & & & & & & & & & & & & & & & \\
\hline \multirow[t]{3}{*}{ C6 } & Not knowing what someone knows & & & & & & & & & & & & & & & & & & 1 & & \\
\hline & Rebuffs from attempts to share knowledge & & & & & & & 1 & & & & & & & & & & & & & \\
\hline & Time to circulate ideas & & & & & & & & 1 & & & & & & & & & & & & \\
\hline \multirow[t]{3}{*}{ C7 } & Team members don't gel & & & & & & & & & & & & 1 & & & & & & & & \\
\hline & Lack of understanding about key outcomes & & & & & & & & & & & & & & & 1 & & & & & \\
\hline & Egos & & & & & & & & & & & 1 & & & & & & & & & \\
\hline \multirow[t]{4}{*}{$\mathrm{CB}$} & Different agendas and loyalties (individual \& organisational) & & & & & & & & & & & & & & & 1 & & & & & \\
\hline & Communication - vital to work together under one roof & & & & & & & 1 & & & & & & & & & & & & & \\
\hline & Commitment of individuals & & & & & & & & & & & & & & & & & & & & 1 \\
\hline & Too many technical skills, not enough relationship building skill & & & & & & & & & & & & & 1 & & & & & & & \\
\hline \multirow[t]{2}{*}{$\mathrm{Cg}$} & Patch protection & & & & & & & & & & 1 & & & & & 1 & & & & & \\
\hline & Misinterpretation of information based on preconceptions & 1 & & & & & & & & & & & & & & & & & & & \\
\hline
\end{tabular}




\begin{tabular}{|c|c|c|c|c|c|c|c|c|c|c|c|c|c|c|c|c|c|c|c|c|c|}
\hline \multirow[b]{2}{*}{ ID } & \multirow[b]{2}{*}{ Barrier } & \multicolumn{20}{|c|}{ Keywords } \\
\hline & & 蒙 & 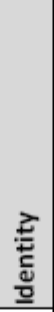 & 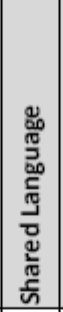 & 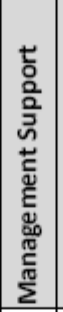 & 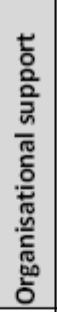 & 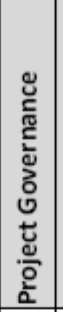 & 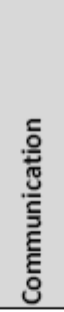 & $\stackrel{\Xi}{\xi}$ & 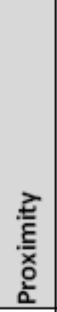 & 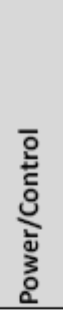 & 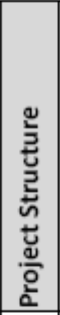 & 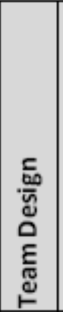 & 音 & \begin{tabular}{|l|} 
\\
है \\
o \\
$z$
\end{tabular} & 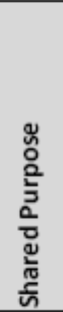 & 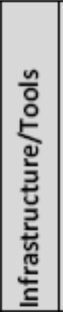 & 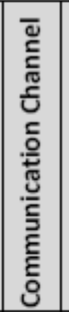 & 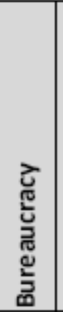 & 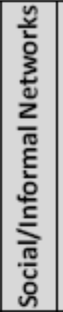 & 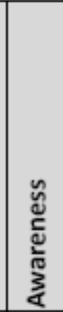 \\
\hline \multirow[t]{3}{*}{ C10 } & Lack of trust & 1 & & & & & & & & & & & & & & & & & & & \\
\hline & Lack of physical proximity to team members & & & & & & & & & 1 & & & & & & & & & & & \\
\hline & Lack of technology & & & & & & & & & & & & & & & & 1 & & & & \\
\hline \multirow[t]{4}{*}{ C11 } & Trust & 1 & & & & & & & & & & & & & & & & & & & \\
\hline & Clarity about individual roles & & & & & & & & & & & 1 & & & & & & & & & \\
\hline & Clarity about shared agenda & & & & & & & & & & & & & & & 1 & & & & & \\
\hline & Time & & & & & & & 1 & & & & & & & & & & & & & \\
\hline \multirow[t]{2}{*}{ C12 } & Trust & 1 & & & & & & & & & & & & & & & & & & & \\
\hline & Personality clashes & & & & & & & 1 & & & & & & & & & & & & & \\
\hline \multirow[t]{3}{*}{ C13 } & Too little time for sharing & & & & & & & & 1 & & & & & & & & & & & & \\
\hline & Synthesising knowledge from different sources & & 1 & & & & & & & & & & & & & & & & & & \\
\hline & Understanding perspectives of others & & & & & & & & & & & & & & & 1 & & & & & \\
\hline \multirow[t]{2}{*}{ C14 } & Shared context & & & & & & & & & & & & & & & 1 & & & & & \\
\hline & Incentives for sharing & & & & & & & & & & & & & & & & & & & 1 & \\
\hline$\overline{D 1}$ & Knowing the right people with the right information & & & & & & & & & & & & 1 & & & & & & & & \\
\hline D2 & Knowing who knows what & & & & & & & & & & & & & & & & & & 1 & & \\
\hline \multirow[t]{3}{*}{ D3 } & Distance & & & & & & & & & 1 & & & & & & & & & & & \\
\hline & Lack of time & & & & & & & & 1 & & & & & & & & & & & & \\
\hline & Worried about what might happen to information & 1 & & & & & & & & & & & & & & & & & & & \\
\hline D4 & Ability to handle large incongruent information sets & & & & & & & & & & & & & & & & 1 & & & & \\
\hline \multirow[t]{2}{*}{ D5 } & Trust & 1 & & & & & & & & & & & & & & & & & & & \\
\hline & Conflict of interests & & & & & & & & & & & & & & & 1 & & & & & \\
\hline \multirow[t]{4}{*}{ D6 } & Individual agendas as opposed to group agenda & & & & & & & & & & & & & & & 1 & & & & & \\
\hline & Lack of open communication & & & & & & & 1 & & & & & & & & & & & & & \\
\hline & Low levels of trust & 1 & & & & & & & & & & & & & & & & & & & \\
\hline & Ensuring participants feel they are part of a team & & & & & & & & & & & & 1 & & & & & & & & \\
\hline
\end{tabular}




\begin{tabular}{|c|c|c|c|c|c|c|c|c|c|c|c|c|c|c|c|c|c|c|c|c|c|}
\hline \multirow[b]{2}{*}{ ID } & \multirow[b]{2}{*}{ Barrier } & \multicolumn{20}{|c|}{ Keywords } \\
\hline & & 营 & 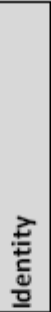 & 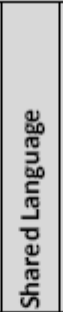 & 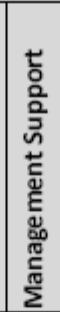 & 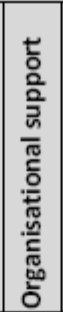 & 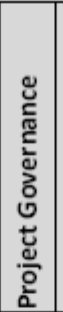 & 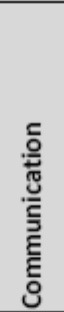 & $\stackrel{\mathscr{\xi}}{\xi}$ & 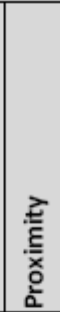 & 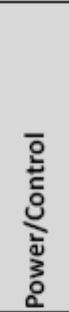 & 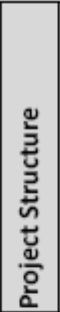 & 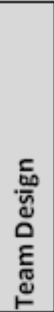 & 竞 & 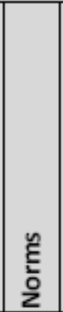 & 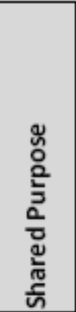 & 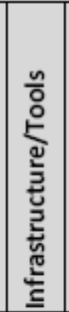 & 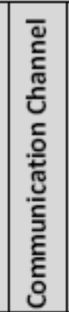 & 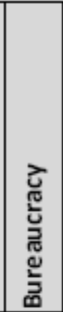 & 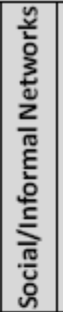 & 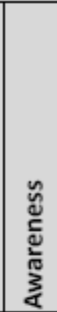 \\
\hline \multirow[t]{2}{*}{ D7 } & Lack of trust & 1 & & & & & & & & & & & & & & & & & & & \\
\hline & Lack of transparency & & & & & & 1 & & & & & & & & & & & & & & \\
\hline \multirow[t]{2}{*}{ D8 } & Lack of time & & & & & & & 1 & & & & & & & & & & & & & \\
\hline & Not agreeing shared purpose and objectives upfront & & & & & & & & & & & & & & & 1 & & & & & \\
\hline \multirow[t]{3}{*}{$\mathrm{Dg}$} & Distrust & 1 & & & & & & & & & & & & & & & & & & & \\
\hline & Insufficient level of commitment from individuals & & & & & & & & & & & & & & & & & & & & 1 \\
\hline & Insufficient opportunities to share, most effective through informal settings & & & & & & & & & & & \begin{tabular}{|l|} 
\\
\end{tabular} & & & & & & & & & \\
\hline \multirow[t]{2}{*}{ D10 } & Lack of trust & 1 & & & & & & & & & & & & & & & & & & & \\
\hline & Poor relationship building & & & & & & & & & & & & 1 & & 1 & & & & & & \\
\hline \multirow[t]{2}{*}{ D11 } & Trust & 1 & & & & & & & & & & & & & & & & & & & \\
\hline & Time & & & & & & & & 1 & & & & & & & & & & & & \\
\hline \multirow[t]{2}{*}{ D12 } & Different organisational agendas & & & & & & & & & & & & & & & 1 & & & & & \\
\hline & Herd mentality & & & & & & & & & & & & & & 1 & & & & & & \\
\hline \multirow[t]{3}{*}{ D13 } & Trust & 1 & & & & & & & & & & & & & & & & & & & \\
\hline & Perceived value and payback for sharing & & & & & & & & & & & & & & & & & & & 1 & \\
\hline & Competition from others & & & & & & & & & & 1 & & & & & & & & & & \\
\hline D14 & Fear of losing face or control of information & 1 & & & & & & & & & 1 & & & & & & & & & & \\
\hline \multirow[t]{3}{*}{ D15 } & Lack of trust & 1 & & & & & & & & & & & & & & & & & & & \\
\hline & No sense of belonging & & 1 & & & & & & & & & & & & & & & & & & \\
\hline & Ineffective delegation & & & & & & 1 & & & & & & & & & & & & & & \\
\hline \multirow[t]{3}{*}{ D16 } & Unwillingness to share - knowledge is power syndrome & & & & & & & & & & 1 & & & & & & & & & & \\
\hline & Lack of trust & 1 & & & & & & & & & & & & & & & & & & & \\
\hline & Different organisational cultures & & & & & & & & & & & & & 1 & & & & & & & \\
\hline
\end{tabular}




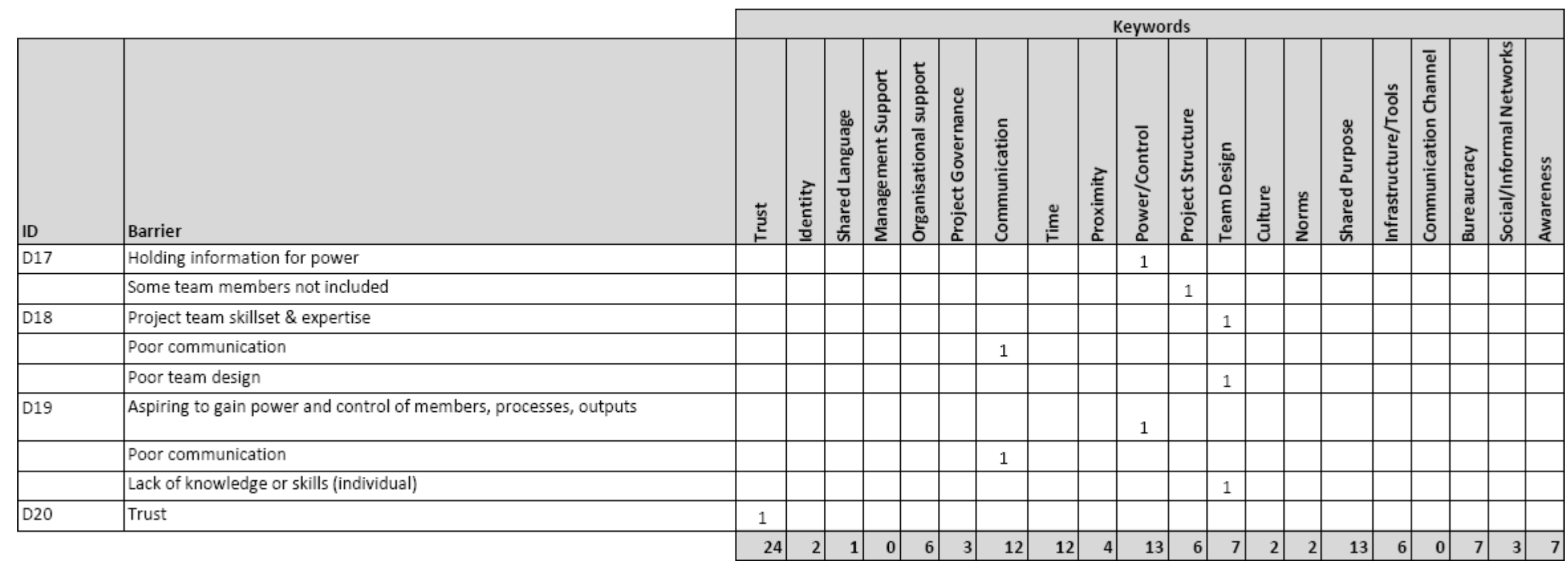

